\title{
C-Terminal Bioconjugation of Peptides through Photoredox Catalyzed Decarboxylative Alkynylation
}

\author{
Marion Garreau, Franck Le Vaillant and Jerome Waser ${ }^{[*]}$
}

\begin{abstract}
We report the first decarboxylative alkynylation of the Cterminus of peptides starting from free carboxylic acids. The reaction is fast, metal-free and proceeds cleanly to afford alkynylated peptides with a broad tolerance for the $\mathrm{C}$-terminal amino acid. Through the use of hypervalent iodine reagents, the introduction of a broad range of functional groups was successful. C-terminal selectivity was achieved by differentiation of the oxidation potentials of the carboxylic acids based on the use of fine-tuned organic dyes.
\end{abstract}

Peptides are important in drug discovery, with 140 peptide therapeutics in clinical trials in $2015{ }^{[1]}$ The biological activity and stability of peptides can be further improved by the synthesis of bioconjugates $^{[2]}$ and macrocycles. ${ }^{[3]}$ There is thus a need for amino acid selective modification methods. ${ }^{[4]}$ Progress has been realized through incorporation of unnatural amino acids. ${ }^{[4,5]}$ However, this strategy implies to know the targeted sequence and requires building blocks compatible with solid-phase synthesis. In contrast, the late-stage functionalization of peptides does not require specialized synthesis methods, but excellent chemoselectivity under mild conditions is needed. ${ }^{4,6]}$ If numerous methods for selective modification of lysine, cysteine, or the $\mathrm{N}$ terminal position have been developed, ${ }^{[7]}$ direct and selective functionalization of the C-terminus remains underexplored. Until recently, available methods relied on amidation reactions or thioester formation. ${ }^{[8]}$ The selectivity issues encountered between the side-chains of aspartic and glutamic acids and the C-terminal position have limited the use of these strategies.

The emergence of photoredox catalysis has led to novel strategies in biomolecule functionalization, due to mild conditions to generate radicals. ${ }^{[9]}$ Decarboxylation strategies were developed for C-terminal functionalization (Scheme 1A). ${ }^{[10 a, b]}$ Starting from free carboxylic acids, arylation, ${ }^{[10 \mathrm{c}, \mathrm{d}]}$ reduction, ${ }^{[10 e, f]}$

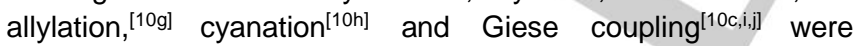
described. The latter was employed by the MacMillan group to selectively functionalize the $\mathrm{C}$-terminal position of native peptides, taking advantage of the different oxidation potentials of the carboxylic acids. ${ }^{[10 k]}$ Selective functionalizations of peptides were reported using redox-active esters (RAE).$^{[11 a-c]} A$ decarboxylative alkynylation was described by Baran and co-workers (Scheme 1B). ${ }^{[11 d]}$ This approach required pre-functionalization of the carboxylic acid and was employed only for side-chain

[a] Marion Garreau, Franck Le Vaillant and Prof. Dr. Jérôme Waser Laboratory of Catalysis and Organic Synthesis

Ecole Polytechnique Fédérale de Lausanne

EPFL SB ISIC LCSO, BCH 4306, 1015 Lausanne $(\mathrm{CH})$

Fax: (+)41216939700

E-mail: jerome.waser@epfl.ch

Homepage: http://lcso.epfl.ch/

Supporting information for this article is given via a link at the end of the document. modification. A direct decarboxylative alkynylation on peptides starting from free carboxylic acids has not yet been achieved.

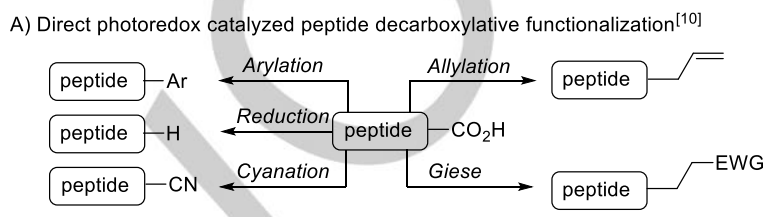

B) Decarboxylative alkynylation of peptides with $\operatorname{RAE}^{[11]}$

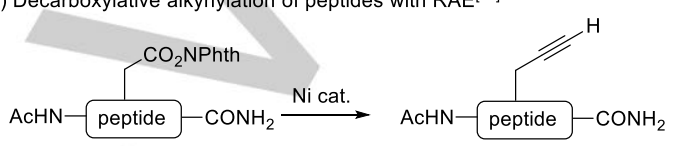

C) Decarboxylative alkynylation of free carboxylic acids (Waser, 2015) ${ }^{[12]}$

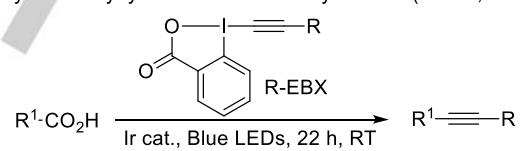

D) THIS WORK: Decarboxylative alkynylation of peptide C-terminus

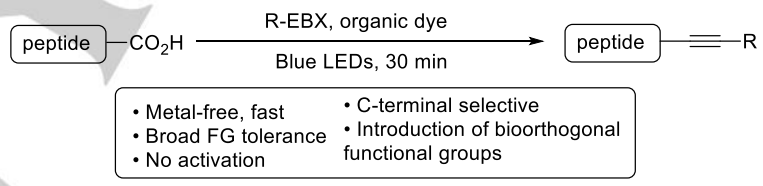

Scheme 1. Photoredox-catalyzed decarboxylation strategies for the functionalization of peptides. EWG = Electron-withdrawing group, Phth = phthaloyl, FG = functional group.

Our group and others developed photoredox-catalyzed decarboxylative alkynylation of amino acids using ethynylbenziodoxolone (EBX) reagents (Scheme 1C). ${ }^{[12]}$ Peptides could not be functionalized in this work. Nevertheless, EBX reagents have a high potential for biomolecule functionalization. They have been used for the alkynylation of thiols in cysteines ${ }^{[13]}$ or indoles in tryptophans. ${ }^{[14]}$ Herein, we report a decarboxylative alkynylation on peptides, which proceeds in $30 \mathrm{~min}$ at room temperature under blue LEDs irradiation using EBX reagents and organic dyes as photocatalysts (Scheme 1D). The reaction was selective for the $\mathrm{C}$-terminus, even in the presence of aspartic or glutamic acid residues.

To avoid the use of expensive and toxic transition metals, we focused on organic dyes, ${ }^{[15]}$ in contrast to previous works based on iridium photocatalysts. ${ }^{[12 a, b]}$ Our group has applied derivatives of 2,4,5,6-tetra(9H-carbazol-9-yl)isophthalonitrile (4CzIPN, 2a) in photoredox catalysis. ${ }^{[16]}$ The introduction of chlorine atoms on the carbazoles (2b) gave stronger oxidizing dyes, closing a gap in potential between 4CzIPN (2a) and dicyanoanthracene (DCA) (+ 1.35 and $2.06 \mathrm{~V}$, Figure 1). Considering the oxidation potentials of terminal vs-side chain carboxylic acids, it is possible to achieve 
C-terminus selectivity (proline carboxylate: $+0.95 \mathrm{~V}$, glycine carboxylate: $+1.20 \mathrm{~V}$, primary amino acids: up to $+1.45 \mathrm{~V}) .{ }^{[17]}$ Easy to oxidize side-chains have also to be taken into consideration, such as tyrosine $(+1.1-1.27 \mathrm{~V})$ and tryptophan $(+0.77-1.16 \mathrm{~V}) .{ }^{[17,18]}$ We were therefore interested in synthesizing organic dyes with reduction potential lower than $+1.35 \mathrm{~V}$, based on the reported 2,4,5,6-tetrakis(diphenylamino)isophthalonitrile (DPAIPN, 3a) $(+1.10 \mathrm{~V})^{[19]}$ to be close to the frequently used

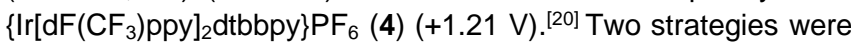
envisaged: adding electron-donating groups on $4 \mathrm{CzIPN}(\mathbf{2 a})$ to lower its potential and halogen substituents on DPAIPN (3a) to make it more oxidizing.
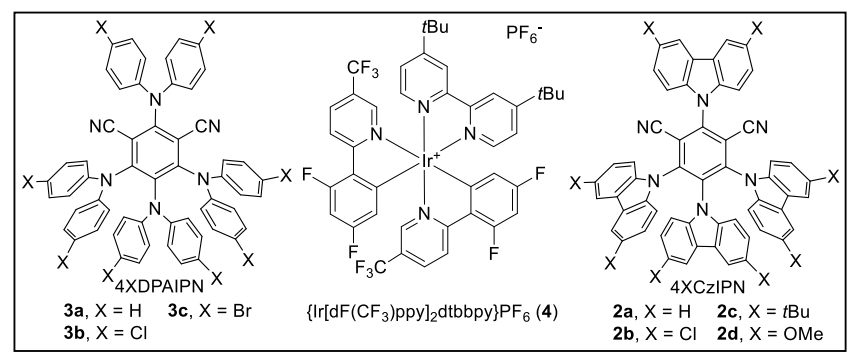

$3 a, X=H$
$3 b, X=C$

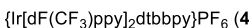

$2 c, X=t B u$

$\mathrm{H}$ 3c, $\mathrm{X}=\mathrm{Br}$

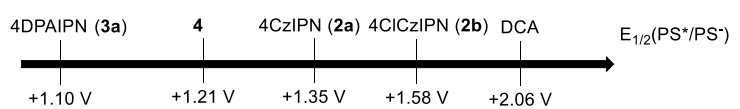

$$
\begin{aligned}
& +1.10 \mathrm{~V} \quad+1.21 \mathrm{~V} \quad+1.35 \mathrm{~V} \quad+1.58 \mathrm{~V} \quad+2.06 \mathrm{~V}
\end{aligned}
$$

Figure 1. Excited state reduction potentials of reported photoredox catalysts $\mathrm{PS}=$ photosensitizer

Starting from 4CzIPN (2a), the introduction of electrondonating groups in para position on the carbazoles led to the dyes 4 tBuCzIPN (2c) ${ }^{[21]}$ and $4 \mathrm{MeOC} z I P N(2 d) .{ }^{[22]}$ We then turned to modification of 4DPAIPN (3a). However, the reported synthesis could not be reproduced. ${ }^{[19]}$ Zeitler and co-workers later showed that the reported conditions led to the incorporation of only three diphenylamine units. ${ }^{[22]}$ Nevertheless, changing the reaction solvent from tetrahydrofuran (THF) to dimethylformamide (DMF) afforded dye 3a. ${ }^{[23]}$ Substitution with electron-withdrawing groups led to 4CIDPAIPN (3b) and 4BrDPAIPN (3c). The redox properties of the dyes were then measured. ${ }^{[24]} 4 t \mathrm{BuCzIPN}$ (2c) and $4 \mathrm{MeOCzIPN}(\mathbf{2 d})$ were less oxidizing at the excited state than 4CzIPN (2a), and 4BrDPAIPN (3c) and 4CIDPAIPN (3b) more oxidizing than 4DPAIPN (3a) (Scheme 2). Interestingly, the "real" 4DPAIPN (3a) was less oxidizing than the dye having only three carbazole units $(+0.90 \mathrm{~V}$ vs $+1.10 \mathrm{~V}) .{ }^{[19]}$ As a result of this work, organic dyes are now available spanning the range of reduction potentials between +0.90 and $+1.35 \mathrm{~V}$.

We investigated then the decarboxylative alkynylation of the dipeptide Cbz-Gly-Pro (5a) (Scheme 2). Using TIPS-EBX (1a, TIPS = triisopropylsilyl) and Ir catalyst 4 led to decomposition of the reagent, which was faster than the desired alkynylation. ${ }^{[12 a]}$ To our delight, using Ph-EBX (1b) allowed isolation of alkynylated dipeptide $6 \mathbf{a}$ with a broad range of catalysts. Due to the reaction mechanism involving a radical intermediate, ${ }^{[10 \mathrm{~h}]} \mathbf{6} \mathbf{6}$ was obtained as a racemic mixture. Best results were obtained using degassed DMF and $\mathrm{K}_{2} \mathrm{HPO}_{4} \cdot{ }^{[10 \mathrm{j}, 24]}$ The stronger oxidant 4CICzIPN (2b) afforded $89 \%$ yield. The reaction was cleaner and gave a quantitative yield in 30 minutes with 4 CzIPN (2a). Using nondegassed solutions still gave $\mathbf{6 a}$ in $87 \%$ yield. The introduction of electron-rich substituents (2c and $\mathbf{2 d}$ ) led to lower yields. The inefficient behavior of $\mathbf{2} \mathbf{d}$ has been described by Zeitler and coworkers and attributed to a fast intramolecular (back) electron transfer from the excited charge transfer (CT) state. ${ }^{[23]}$ For $\mathbf{2 c}$, we observed full consumption of the catalyst, indicating stability issues. Increasing the catalyst loading to $5 \mathrm{~mol} \%$ afforded $57 \%$ yield. Using iridium catalyst $\mathbf{4}$ with similar reduction potential, alkyne 6 a was obtained in quantitative yield. Yields decreasing from 65 to 50 to $43 \%$ were obtained for 4DPAIPN dyes 3a-c, correlating with decreasing reduction potentials. We selected 4CzIPN (2a) for studying the scope, keeping in mind that lessoxidizing dyes could be used in case of selectivity issues.

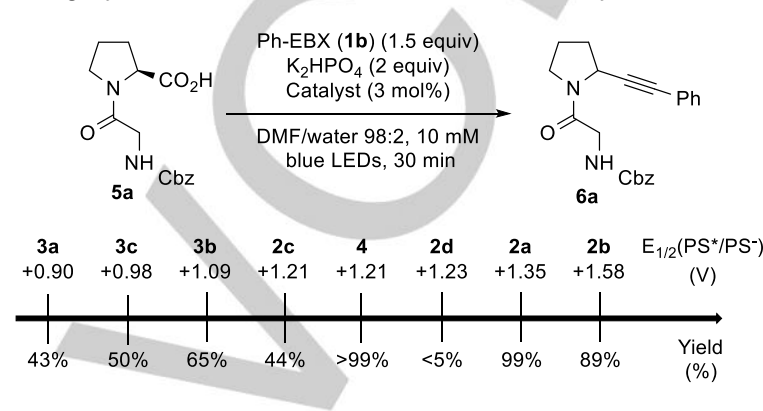

Scheme 2. Screening of photocatalysts for the alkynylation of peptide 5 a.

The scope of EBX reagents was evaluated first (Scheme 3A). Electron-withdrawing groups were tolerated in para position on the benzene, including $\mathrm{CF}_{3}$, bromine, aldehyde or cyanide (6b-e). An electron-donating pentyl chain was also tolerated (6f). Variation of the position on the aryl ring was possible (meta fluoro and ortho bromo substituents in $\mathbf{6 g}$ and $\mathbf{6 h}$ ). New EBX reagents bearing biorthogonal azide or free terminal alkyne groups could be used successfully and $\mathbf{6 i}$ and $\mathbf{6 j}$ were obtained both in $67 \%$ yield. The methodology was applied to vinyl-benziodoxolone (VBX) reagent $7^{[25]}$ to afford 8 in $82 \%$ yield as the $E$ isomer. The use of alkyl or silyl substituted EBXs led to only very low yields.

Dipeptides were then investigated (Scheme 3B). Changing the second amino acid with a terminal proline had little influence. Starting from Cbz-Phe-Pro ( $\mathrm{Cbz}=$ carboxybenzyl), alkyne 9a was isolated in $79 \%$ yield as a $2: 1$ mixture of diastereoisomers. When phenylalanine or alanine were at the C-terminal position, the alkynylated products $9 \mathrm{~b}$ and $9 \mathrm{c}$ were obtained in 68 and $79 \%$ yield, the latter as a 1:1 mixture of diastereoisomers. For glycine, conversion was not complete and alkyne $9 \mathbf{d}$ was isolated in $25 \%$ yield. Although $4 \mathrm{CzIPN}(\mathbf{2 a})$ is thermodynamically able to oxidize glycine carboxylate salts $(+1.35$ vs $1.20 \mathrm{~V})$, it is important to remember that many redox processes have significant overpotential. Indeed, switching to the more oxidizing dye 4 CICzIPN (2b) improved the yield to $58 \%$. 


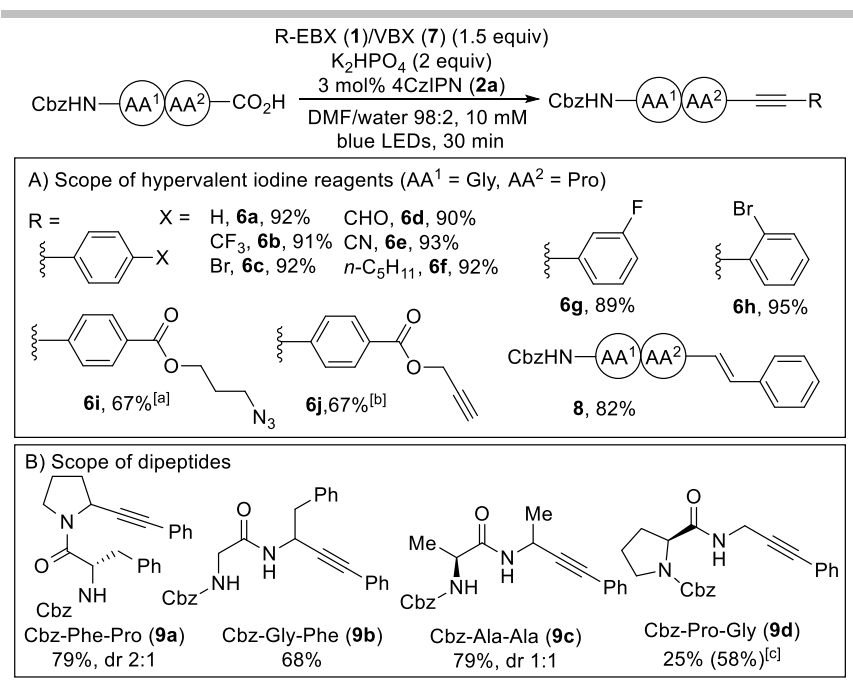

Scheme 3. Scope of the reaction on dipeptides. Reaction conditions: peptide (1.0 equiv, $0.3 \mathrm{mmol}$ ), 4CzIPN (2a) (3 mol\%), R-EBX (1) (1.5 equiv) and $\mathrm{K}_{2} \mathrm{HPO}_{4}$ (2 equiv) in DMF/water $98: 2(10 \mathrm{mM})$ at $\mathrm{rt}$ for $30 \mathrm{~min}$. Isolated yields are given ${ }^{[a]} \mathrm{On} 0.05 \mathrm{mmol}$ scale. ${ }^{[\mathrm{b}]} \mathrm{On} 0.1 \mathrm{mmol}$ scale. ${ }^{[c]}$ With $4 \mathrm{ClCzIPN}(\mathbf{2 b})$ as catalyst.

Before expanding the scope to larger peptides, we performed a robustness test: ${ }^{[26]}$ the alkynylation of dipeptide 5 a was repeated in presence of functionalized amino acids, and only for the most electron-rich ones a lower yield was observed. ${ }^{[24]}$ We then examined variation at the $\mathrm{N}$-terminus of peptide tetramers bearing a C-terminal proline (Scheme 4A). After slight modification of the conditions (increasing of the excess of reagent, base and catalyst loading), full and clean conversion to alkynylated peptides 11a and $\mathbf{1 1 b}$ was obtained with alanine or arginine at the $\mathrm{N}$-terminus. Amino acids with basic side-chains such as histidine and lysine led to a lower yield (11c and 11d). With tyrosine and tryptophan, $11 \mathrm{e}$ and $11 \mathrm{f}$ could not be detected. ${ }^{[27]}$ Gratifyingly, changing the catalyst to the less oxidizing $\left\{\operatorname{Ir}\left[\mathrm{dF}\left(\mathrm{CF}_{3}\right) \text { ppy }\right]_{2} \mathrm{dtbbpy}_{\mathrm{PF}_{6}}(\mathbf{4})\right.$, allowed the formation of peptide 11e in $54 \%$ yield. 4 tBuCzIPN (2c) afforded $20 \%$ of yield and the use of 4DPAIPN-derived dyes 3a-c led to only traces of product 11e. This approach was not successful with tryptophan, but carrying the reaction prior to deprotection of the indole led to $89 \%$ yield. In presence of a cysteine, a very fast thiol-alkynylation takes place first, affording bis-functionalized alkyne $\mathbf{1 1 g}$ quantitatively. The use of trityl protected peptide gave C-terminus alkynylation in 94\% yield.

Variation of the C-terminus was then investigated (Scheme $4 \mathrm{~B}$ ). Full and clean conversion to peptides $11 \mathrm{~h}-\mathrm{m}$ was obtained with alanine, phenylalanine, arginine, serine, methionine, asparagine and glycine. A lower yield was observed for histidine, lysine or tyrosine (110-q). When lysine was protected, the yield was quantitative. Aspartic and glutamic acid were tolerated at the C-terminus. Full and clean conversion was obtained for glutamic acid (11r), and $37 \%$ of the mono-alkynylated peptide $11 \mathrm{~s}$ with aspartic acid. C-terminus selectivity with side-chain carboxylic acids at $\mathrm{N}$-terminus was then investigated (Scheme $4 \mathrm{C}$ ). In the presence of aspartic acid, selective C-terminus alkynylation was observed in the case of proline, alanine and even glycine with good to excellent conversion (11t to 11v). Alkynylation at the side chain of aspartic or glutamic acid was never observed in more than $5 \%$. Similar reactivity and selectivity were obtained with an $\mathrm{N}$-terminus glutamic acid (11w and $\mathbf{1 1 x})$.

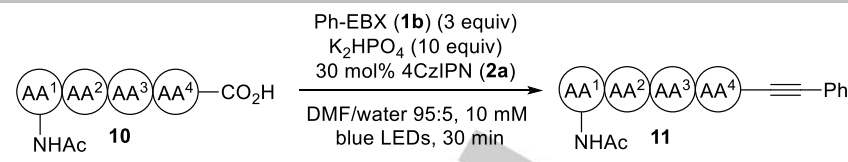

\begin{tabular}{|c|c|}
\hline 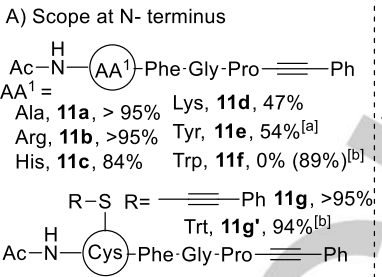 & 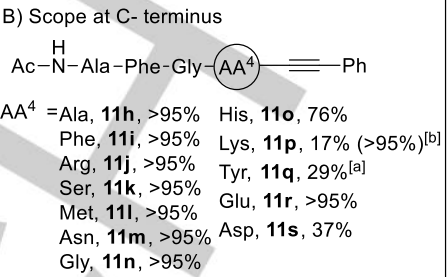 \\
\hline
\end{tabular}

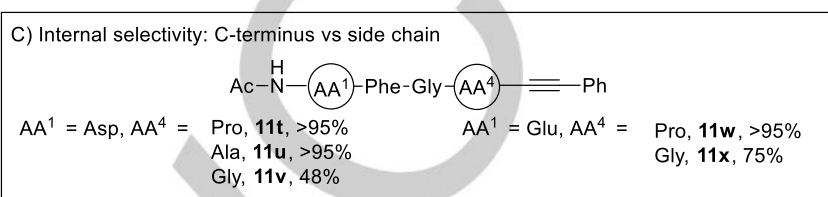

Scheme 4. Scope of the reaction on peptide tetramers. Reaction conditions: peptide (1.0 equiv, $1 \mu \mathrm{mol}), 4 \mathrm{CzIPN}(\mathbf{2 a})(30 \mathrm{~mol} \%), \mathrm{Ph}-\mathrm{EBX}$ (1b) (3 equiv) and $\mathrm{K}_{2} \mathrm{HPO}_{4}$ (10 equiv) in DMF/water 95:5 $(10 \mathrm{mM})$ at $\mathrm{rt}$ for $30 \mathrm{~min}$. Yield measured by HPLC as the ratio of the area of the product over remaining starting material and possible side-products at $214 \mathrm{~nm}$. Average of 3 independent trials. All peptides were obtained as non-separable mixtures of diastereoisomers at the

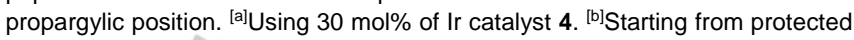
peptide.

The methodology was then applied to the $\mathrm{N}$-terminal protected hexamer GRGDNP (12a), a potent inhibitor of cell attachment to fibronectin, ${ }^{[2]}$ which was successfully functionalized with EBX reagents bearing an azide or an aldehyde functional group to give 13a and 13b (Scheme 5). Alkynylation of N-terminus unprotected peptide 12c and 12d was also possible, even if no complete conversion was achieved: Peptides $13 \mathrm{c}$ and 13d bearing bromide and azide functionalities were obtained in 52 and $57 \%$ yield.

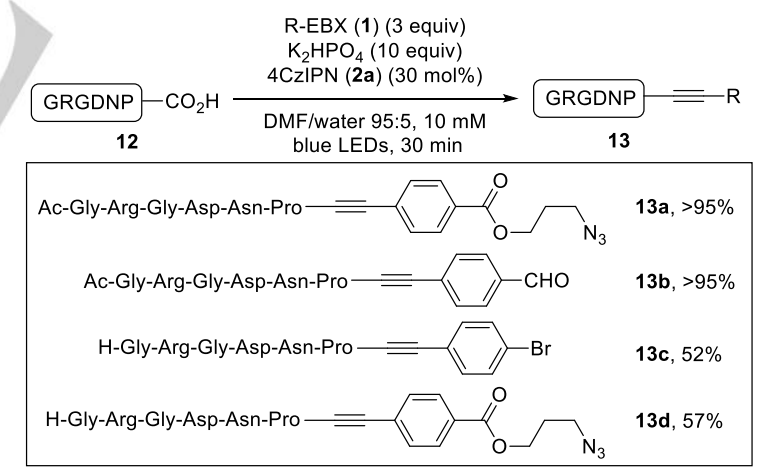

Scheme 5. Functionalization of GRGDNP (12). Reaction conditions: peptide (1 equiv, $1 \mu \mathrm{mol}$ ), 4CzIPN (2a) (30 mol\%), R-EBX (1) (3 equiv) and $\mathrm{K}_{2} \mathrm{HPO}_{4}$ (10 equiv) in DMF/water 95:5 (10 mM) at $\mathrm{rt}$ for $30 \mathrm{~min}$. Yield measured by HPLC as the ratio of the area of the product over remaining starting material and possible side-products at $214 \mathrm{~nm}$. Average of 3 independent trials. All peptides were obtained as non-separable mixtures of diastereoisomers at the propargylic position.

In summary, a metal-free decarboxylative alkynylation of the C-terminus of peptides has been developed. Using organic dyes, alkynylated products were obtained in 30 minutes at room temperature. Complete selectivity for the C-terminus was observed in presence of carboxylic acid side-chains. Most other side-chains were also tolerated, with the exception of tryptophan, 
which needed to be protected, resulting in a broadly applicable methodology for modifying peptides.

\section{Acknowledgements}

We thank the ERC (European Research Council, Starting Grant iTools4MC, number 334840), NCCR Chemical Biology (SNSF) and EPFL for financial support. The MS service from EPFL-ISIC is acknowledged for their support. We thank Dr R. Scopelliti and Dr F. F. Tirani from ISIC at EPFL for X-ray analysis. MG thanks Ophélie Planes (LCS, EPFL) for her precious help with CV experiments. Romain Tessier and Dr J. De Ceballos from LCSO are acknowledged for their help through meaningful discussions.

Keywords: peptides, photoredox catalysis, hypervalent iodine, late-stage functionalization, organic dyes.

[1] a) K. Fosgerau, T. Hoffmann, Drug Discov. Today 2015, 20, 122; b) I. W. Hamley, Chem. Rev. 2017, 117, 14015; c) N. Qvit, S. J. S. Rubin, T. J. Urban, D. Mochly-Rosen, E. R. Gross, Drug Discov. Today 2017, 22, 454

[2] a) Q. Y. Hu, F. Berti, R. Adamo, Chem. Soc. Rev. 2016, 45, 1691; b) S. B. Gunnoo, A. Madder, Org. Biomol. Chem. 2016, 14, 8002.

[3] a) C. J. White, A. K. Yudin, Nat. Chem. 2011, 3, 509; b) T. A. Hill, N. E. Shepherd, F. Diness, D. P. Fairlie, Angew. Chem. Int. Ed. 2014, 53, $13020 ;$ c) Y. H. Lau, P. De Andrade, Y. T. Wu, D. R. Spring, Chem. Soc Rev. 2015, 44, 91.

[4] a) C. P. R. Hackenberger, D. Schwarzer, Angew. Chem., Int. Ed. 2008 47, 10030; b) C. D. Spicer, B. G. Davis, Nat. Commun. 2014, 5, 4740; c) O. Boutureira, G. J. L. Bernardes, Chem. Rev. 2015, 115, 2174; d) O Koniev, A. Wagner, Chem. Soc. Rev. 2015, 44, 5495.

[5] a) Y. Ryu, P. G. Schultz, Nat. Methods 2006, 3, 263; b) E. M. Sletten, C. R. Bertozzi, Angew. Chem. Int. Ed. 2009, 48, 6974.

[6] a) E. Baslé, N. Joubert, M. Pucheault, Chem. Biol. 2010, 17, 213; b) T. Brandhofer, O. García Mancheño, Eur. J. Org. Chem. 2018, 2018, 6050 c) W. Wang, M. M. Lorion, J. Shah, A. R. Kapdi, L. Ackermann, Angew. Chem., Int. Ed. 2018, 57, 14700.

[7] a) J. N. DeGruyter, L. R. Malins, P. S. Baran, Biochemistry 2017, 56 3863; b) C. B. Rosen, M. B. Francis, Nat. Chem. Biol. 2017, 13, 697.

[8] a) M. A. Gilles, A. Q. Hudson, C. L. Borders, Anal. Biochem. 1990, 184, 244; b) L. Yi, H. Sun, Y.-W. Wu, G. Triola, H. Waldmann, R. S. Goody, Angew. Chem. Int. Ed. 2010, 49, 9417; c) K. A. Mix, R. T. Raines, Org Lett. 2015, 17, 2358; d) B. Wu, H. J. Wijma, L. Song, H. J. Rozeboom, C. Poloni, Y. Tian, M. I. Arif, T. Nuijens, P. J. L. M. Quaedflieg, W Szymanski, B. L. Feringa, D. B. Janssen, ACS Catal. 2016, 6, 5405; e) P. Martín-Gago, E. K. Fansa, M. Winzker, S. Murarka, P. Janning, C. Schultz-Fademrecht, M. Baumann, A. Wittinghofer, H. Waldmann, Cell Chem. Biol. 2017, 24, 589.

[9] a) C. Hu, Y. Chen, Tetrahedron Lett. 2015, 56, 884; b) C. Bottecchia, T. Noël, Chem. Eur. J. 2019, 25, 26.

[10] Review: a) S. Mondal, S. Chowdhury, Adv. Synth. Catal. 2018, 360 1884; b) L. R. Malins, Pept. Sci. 2018, 110, e24049; Selected examples: c) K. Maeda, H. Saito, K. Osaka, K. Nishikawa, M. Sugie, T. Morita, I. Takahashi, Y. Yoshimi, Tetrahedron 2015, 71, 1117; d) A. Lipp, G. Lahm T. Opatz, J. Org. Chem. 2016, 81, 4890; e) T. Itou, Y. Yoshimi, K. Nishikawa, T. Morita, Y. Okada, N. Ichinose, M. Hatanaka, Chem. Commun. 2010, 46, 6177; f) C. Cassani, G. Bergonzini, C. J. Wallentin, Org. Lett. 2014, 16, 4228; g) S. B. Lang, K. M. O'Nele, J. T. Douglas, J. A. Tunge, Chem. A Eur. J. 2015, 21, 18589; h) F. Le Vaillant, M. D. Wodrich, J. Waser, Chem. Sci. 2017, 8, 1790; i) L. Chu, C. Ohta, Z. Zuo D. W. C. MacMillan, J. Am. Chem. Soc. 2014, 136, 10886; j) S. J. McCarver, J. X. Qiao, J. Carpenter, R. M. Borzilleri, M. A. Poss, M. D. Eastgate, M. M. Miller, D. W. C. MacMillan, Angew. Chem. Int. Ed. 2017 56, 728; k) S. Bloom, C. Liu, D. K. Kölmel, J. X. Qiao, Y. Zhang, M. A Poss, W. R. Ewing, D. W. C. MacMillan, Nat. Chem. 2017, 10, 205 During completion of this manuscript, a first example of decarboxylative azidation has also appeared: I) D. C. Marcote, R. Street-Jeakings, E. Dauncey, J. J. Douglas, A. Ruffoni, D. Leonori, Org. Biomol. Chem. 2019 $17,1839$.

[11] a) T. Qin, J. Cornella, C. Li, L. R. Malins, J. T. Edwards, S. Kawamura B. D. Maxwell, M. D. Eastgate, P. S. Baran, Science 2016, 352, 801; b) J. T. Edwards, R. R. Merchant, K. S. McClymont, K. W. Knouse, T. Qin, L. R. Malins, B. Vokits, S. A. Shaw, D.-H. Bao, F.-L. Wei, T. Zhou, M. D. Eastgate, P. S. Baran, Nature 2017, 545, 213; c) T. Qin, L. R. Malins, J. T. Edwards, R. R. Merchant, A. J. E. Novak, J. Z. Zhong, R. B. Mills, M. Yan, C. Yuan, M. D. Eastgate, P. S. Baran, Angew. Chem. Int. Ed. 2016, 56, 260; d) J. M. Smith, T. Qin, R. R. Merchant, J. T. Edwards, L. R. Malins, Z. Liu, G. Che, Z. Shen, S. A. Shaw, M. D. Eastgate, P. S. Baran, Angew. Chem. Int. Ed. 2017, 56, 11906.

[12] a) F. Le Vaillant, T. Courant, J. Waser, Angew. Chem. Int. Ed. 2015, 54 11200 ; b) Q. Q. Zhou, W. Guo, W. Ding, X. Wu, X. Chen, L. Q. Lu, W. J. Xiao, Angew. Chem. Int. Ed. 2015, 54, 11196; c) C. Yang, J.-D. Yang, Y.-H. Li, X. Li, J.-P. Cheng, J. Org. Chem. 2016, 81, 12357; For examples of photoredox decarboxylative alkynylation starting from RAE, see: d) J. Yang, J. Zhang, L. Qi, C. Hu, Y. Chen, Chem. Commun. 2015, 51, 5275; e) H. Zhang, P. Zhang, M. Jiang, H. Yang, H. Fu, Org. Lett. 2017, 19, 1016; f) J. Schwarz, B. König, ChemPhotoChem 2017, 1, 237.

[13] a) R. Frei, M. D. Wodrich, D. P. Hari, P. A. Borin, C. Chauvier, J. Waser J. Am. Chem. Soc. 2014, 136, 16563; b) D. Abegg, R. Frei, L. Cerato, D. Prasad hari, C. Wang, J. Waser, A. Adibekian, Angew. Chem. Int. Ed. 2015, 54, 10852.

[14] a) G. L. Tolnai, J. P. Brand, J. Waser, Beilstein J. Org. Chem. 2016, 12, 745; b) M. B. Hansen, F. Hubálek, T. Skrydstrup, T. Hoeg-Jensen, Chem Eur. J. 2016, 22, 1572

[15] a) N. A. Romero, D. A. Nicewicz, Chem. Rev. 2016, 116, 10075; b) D. P. Hari, B. König, Chem. Commun. 2014, 50, 6688; c) S. Fukuzumi, K. Ohkubo, Org. Biomol. Chem. 2014, 12, 6059; d) I. Ghosh, R. S. Shaikh, B. König, Angew. Chem. Int. Ed. 2017, 56, 8544

[16] a) F. Le Vaillant, M. Garreau, S. Nicolai, G. Gryn'Ova, C. Corminboeuf, J. Waser, Chem. Sci. 2018, 9, 5883.

[17] a) Z. Zuo, D. W. C. Macmillan, J. Am. Chem. Soc. 2014, 136, 5257; b) H. G. Roth, N. A. Romero, D. A. Nicewicz, Synlett 2016, 27, 714. To facilitate comparison, all redox values are given vs SCE. Literature values given with other reference electrodes have been converted.

[18] a) A. Harriman, J. Phys. Chem. 1987, 91, 6102; b) M. DeFelippis, C. Murthy, J. Phys. Chem. 1991, 8, 3416. c) F. Costanzo, M. Sulpizi, R. G. D. Valle, M. Sprik, J. Chem. Phys. 2011, 134, 244508; d) A. Roy, R Seidel, G. Kumar, S. E. Bradforth, J. Phys. Chem. B 2018, 122, 3723; e) D. M. Close, P. Wardman, J. Phys. Chem. A 2018, 122, 439.

[19] J. Luo, J. Zhang, ACS Catal. 2016, 6, 873.

[20] M. S. Lowry, J. I. Goldsmith, J. D. Slinker, R. Rohl, R. A. Pascal, G. G. Malliaras, S. Bernhard, Chem. Mater. 2005, 5712.

[21] a) Y. J. Cho, K. S. Yook, J. Y. Lee, Adv. Mat. 2014, 26, 6642; b) R. Ishimatsu, T. Edura, C. Adachi, K. Nakano, T. Imato, Chem. Eur. J. 2016 22, 4889.

[22] E. Speckmeier, T. Fischer, K. Zeitler, J. Am. Chem. Soc. 2018, 45, 15353.

[23] The structure of $\mathbf{3} \mathbf{a}$ and $\mathbf{3} \mathbf{b}$ was confirmed by $\mathbf{X}$-ray analysis. The data is available at the Cambridge Crystallographic Data Center (ccdc numbers 1879098 (3a) and 1879097 (3b)).

[24] See Supporting Information for further details.

[25] E. Stridfeldt, A. Seemann, M. J. Bouma, C. Dey, A. Ertan, B. Olofsson, Chem. Eur. J. 2016, 22, 16066.

[26] K. D. Collins, F. Glorius, Nat. Chem. 2013, 5, 597.

[27] In the case of tryptophan no conversion was observed. Electron-transfer to tryptophan is very easy, see ref. $10 \mathrm{k}$ and Y. Yu, L.-K. Zhang, A. V. Buevich, G. Li, H. Tang, P. Vachal, S. L. Colletti, Z.-C. Shi, J. Am. Chem. Soc. 2018, 140, 6797.

[28] a) V. Sarin, R. D. Gaffin, G. A. Meininger, M. Muthuchamy, J. Physiol. 2005, 564, 603; b) A. Sasamoto, M. Nagino, S. Kobayashi, K. Naruse, Y Nimura, M. Sokabe, Am. J. Physiol. Cell Physiol. 2005, 288, C1012. 


\section{COMMUNICATION}

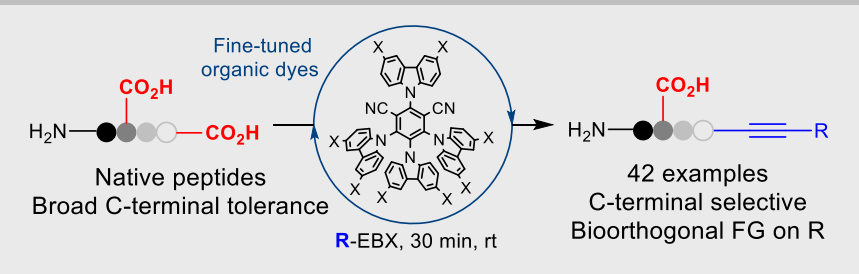

Only at the end: $A$ decarboxylative alkynylation of the $C$-terminal of peptides starting from free carboxylic acids has been developed. The reaction is fast, metal-free and proceeds cleanly to afford alkynylated peptides bearing a broad range of functional groups. C-terminal selectivity was achieved based on the use of fine-tuned organic dyes.
Marion Garreau, Franck Le Vaillant and Jerome Waser*

\section{Page No. - Page No.}

C-Terminal Bioconjugation of Peptides Through Photoredox Catalyzed Decarboxylative Alkynylation 


\section{Table of contents}

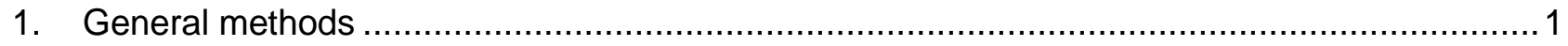

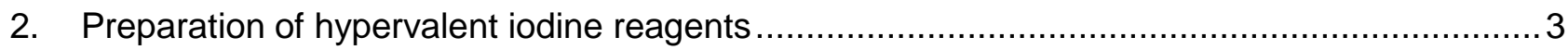

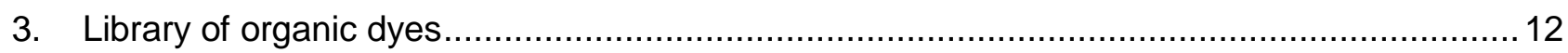

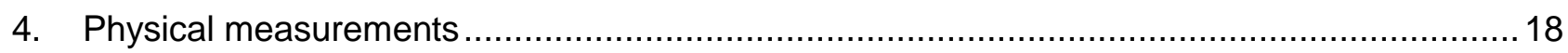

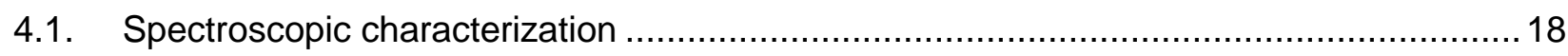

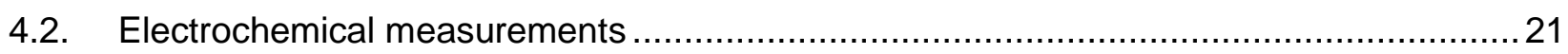

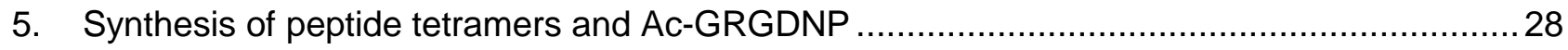

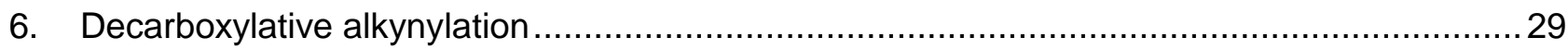

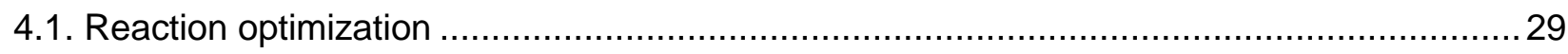

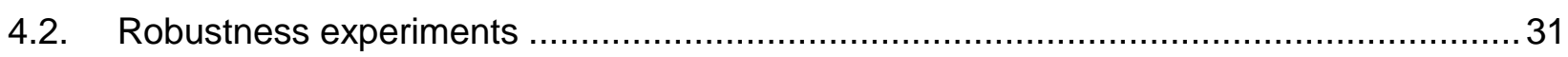

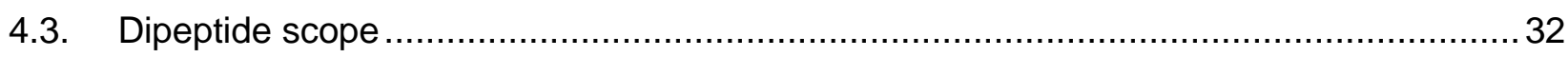

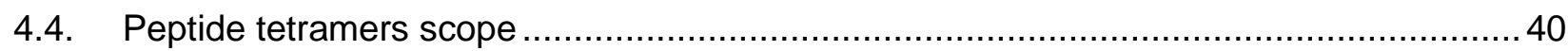

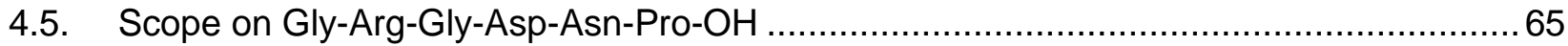

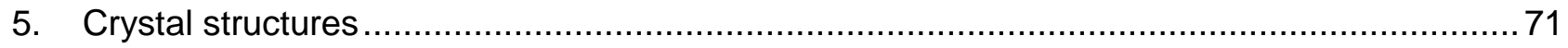

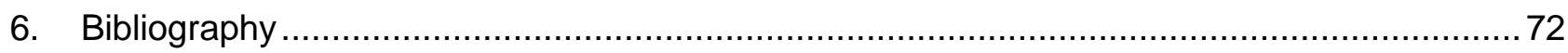

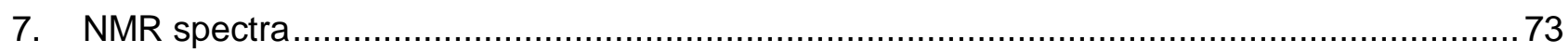




\section{General methods}

All reactions were carried out in oven dried glassware under an atmosphere of nitrogen, unless for the decarboxylative alkynylation and if stated otherwise. For flash chromatography, distilled technical grade solvents were used. THF, $\mathrm{CH}_{3} \mathrm{CN}$, toluene and $\mathrm{CH}_{2} \mathrm{Cl}_{2}$ were dried by passage over activated alumina under nitrogen atmosphere $\left(\mathrm{H}_{2} \mathrm{O}\right.$ content $<10 \mathrm{ppm}$, Karl-Fischer titration). The solvents were degassed by Freeze-Pump-Thaw method when mentioned. All chemicals were purchased from Acros, Aldrich, Fluka, VWR, TCI, Merck or Bachem and used as such unless stated otherwise. All dipeptides starting materials and H-GRGDNP-OH were commercially available and used as received. Chromatographic purification was performed as flash chromatography using MachereyNagel silica 40-63, 60 A, using the solvents indicated as eluent with 0.1-0.5 bar pressure. TLC was performed on Merck silica gel 60 F254 TLC aluminum or glass plates and visualized with UV light. ${ }^{1} \mathrm{H}-\mathrm{NMR}$ spectra were recorded on a Brucker DPX-400 $400 \mathrm{MHz}$ spectrometer in chloroform-d, $\mathrm{CD}_{3} \mathrm{OD}, \mathrm{DMSO}-\mathrm{d}^{6}$ or acetone- $\mathrm{d}^{6}$, all signals are reported in ppm with the internal chloroform signal at $7.26 \mathrm{ppm}$, the internal methanol signal at $3.30 \mathrm{ppm}$, the internal DMSO signal at $2.50 \mathrm{ppm}$ or the internal acetone signal at $2.05 \mathrm{ppm}$ as standard. The data is being reported as $(\mathrm{s}=$ singlet, $\mathrm{d}=$ doublet, $\mathrm{t}=$ triplet, $\mathrm{q}=$ quadruplet, $\mathrm{qi}=$ quintet, $\mathrm{m}=$ multiplet or unresolved, $\mathrm{br}=$ broad signal, $\mathrm{app}=$ apparent, coupling constant(s) in $\mathrm{Hz}$, integration, interpretation). ${ }^{13} \mathrm{C}-\mathrm{NMR}$ spectra were recorded with ${ }^{1} \mathrm{H}$-decoupling on a Brucker DPX-400 $100 \mathrm{MHz}$ spectrometer in chloroform-d, $\mathrm{CD}_{3} \mathrm{OD}$, DMSO$\mathrm{d}^{6}$ or acetone- $\mathrm{d}^{6}$, all signals are reported in ppm with the internal chloroform signal at $77.0 \mathrm{ppm}$, the internal methanol signal at $49.0 \mathrm{ppm}$, the internal DMSO signal at $39.5 \mathrm{ppm}$ or the internal acetone signals at 29.84 and 206.26 ppm as standard. Infrared spectra were recorded on a JASCO FT-IR B4100 spectrophotometer with an ATR PRO410-S and a ZnSe prisma and are reported as cm-1 (w = weak, $\mathrm{m}=$ medium, $\mathrm{s}=$ strong, $\mathrm{br}=$ broad).

High resolution mass spectrometric measurements were performed by the mass spectrometry service of ISIC at the EPFL on a MICROMASS (ESI) Q-TOF Ultima API. MS-MS analyses were performed on a LTQ Orbitrap FTMS instrument (LTQ Orbitrap Elite FTMS, Thermo Scientific, Bremen, Germany) operated in the positive mode coupled with a robotic chip-based nano-ESI source (TriVersa Nanomate, Advion Biosciences, Ithaca, NY, U.S.A.). A standard data acquisition and instrument control system was utilized (Thermo Scientific) whereas the ion source was controlled by Chipsoft 8.3.1 software (Advion BioScience). Samples were loaded onto a 96-well plate (Eppendorf, Hamburg, Germany) within an injection volume of $5 \mu$ l. The experimental conditions for the ionization voltage was $+1.4 \mathrm{kV}$ and the gas pressure was set at $0.30 \mathrm{psi}$. The temperature of ion transfer capillary was $275^{\circ} \mathrm{C}$, tube voltages. FTMS spectra were obtained in the $80-1000 \mathrm{~m} / \mathrm{z}$ range in the reduce profile mode with a resolution set to 120,000. In all spectra one microscan was acquired with a maximum injection time value of $1000 \mathrm{~ms}$. Typical CID experiments were carried out using Normalized collision energy values of $26-28$ and $5 \mathrm{Da}$ of isolation width.

Photoredox catalyzed reactions were performed in test tubes (5 and $10 \mathrm{~mL}$ ), or Schlenk tubes (50 $\mathrm{mL}$ ) which were hold using a rack for test tubes placed at the center of a crystallization flask. For overnight reactions, the latter was filled by water, in order to keep the temperature as constant as possible, in presence of an air flow. On this flask were attached the blue LEDs (RUBAN LED 5MĖTRES - 60LED/M - 3528 BLEU - IP65 with Transformateur pour Ruban LED 24W/2A/12V, bought directly on RubanLED.com). The distance between the LEDs and the test tubes was approximatively $2 \mathrm{~cm}$ for the test tubes and $5 \mathrm{~cm}$ for the Schlenk flasks. Long irradiation resulted in temperature increasing up to $37^{\circ} \mathrm{C}$ during overnight reactions.

Tetramers peptides were synthesized by solid phase peptide synthesis using a Multipep RSi Intavis. Crude products were purified by preparative RP-HPLC on an Agilent 1260 HPLC system with a G2260A 1260 Prep ALS Autosampler, a G1361a 1260 Prep Pump, a G1365C 1260 MWD detector and a G1364B 1260 FC-PS collector, coupled with a Waters XBridge semi-preparative C18 column $(19 \times 150 \mathrm{~mm}, 5 \mu \mathrm{m})$. Water (solvent A) and water:acetonitrile 5:95 (solvent B), each containing $0.1 \%$ TFA, were used as the mobile phase at a flow rate of $20 \mathrm{~mL}$.min-1. The following method was used: $100 \%$ A to $100 \% \mathrm{~B}$ in 20 minutes. 
RP-HPLC-MS measurements were performed on an Agilent 1290 Infinity HPLC system with a G4226a 1290 Autosampler, a G4220A 1290 Bin Pump and a G4212A 1290 DAD detector, connected to a 6130 Quadrupole LC/MS MS, coupled with a Waters XBridge C18 column (250 x 4.6 $\mathrm{mm}, 5 \mu \mathrm{m}$ ). Water:acetonitrile 95:5 (solvent A) and water:acetonitrile 5:95 (solvent B), each containing $0.1 \%$ formic acid, were used as the mobile phase at a flow rate of $0.6 \mathrm{~mL} / \mathrm{min}-1$. The gradient was programmed as follows: $100 \% \mathrm{~A}$ to $100 \% \mathrm{~B}$ in 20 minutes then isocratic for 5 minutes (method A). The column temperature was set up to $25^{\circ} \mathrm{C}$. Low resolution mass spectrometric measurements were acquired using the following parameters: positive electrospray electrospray ionization $(\mathrm{ESI})$, temperature of drying gas $=350^{\circ} \mathrm{C}$, flow rate of drying gas $=12 \mathrm{~L}$. min- 1 , pressure of nebulizer gas $=60 \mathrm{psi}$, capillary voltage $=2500 \mathrm{~V}$ and fragmentor voltage $=70 \mathrm{~V}$. In few cases, the gradient was programmed as follows: $100 \%$ A to $100 \%$ B in 40 minutes, in order to resolve peak overlapping (method B). The other parameters were untouched.

UV/Vis spectroscopy was performed on an Agilent Cary 60 UV-Vis and steady-state luminescence spectroscopy was recorded on a Varian Cary Eclipse spectrophotometer. Cyclic voltammetry experiments were performed on a Biologic SP-150 Potentiostat, with a three-electrode cell configuration: a glassy carbon electrode as the working electrode, $\mathrm{Pt}$ wire as a counter electrode and an $\mathrm{Ag} / \mathrm{AgCl}(\mathrm{KCl}, 3 \mathrm{M})$ electrode as the reference electrode. $\mathrm{Bu}_{4} \mathrm{NPF}_{6}$ was employed as the electrolyte $(0.1 \mathrm{M})$. 


\section{Preparation of hypervalent iodine reagents}

The synthesis of reagents $\mathbf{1 a}-\mathbf{1 h}$ had already been described before by our group. The procedures are taken from the indicated publications to facilitate reproduction of the results by having all data in the same file.

\section{1-Hydroxy-1,2-benziodoxol-3-(1H)-one (15)}

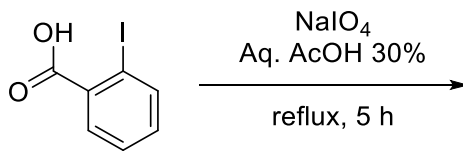

14<smiles>O=c1oc2ccccc2o1</smiles>

15

Following a reported procedure, ${ }^{[1]} \mathrm{NalO}_{4}(40.5 \mathrm{~g}, 189 \mathrm{mmol}, 1.05$ equiv) and 2-iodobenzoic acid (14) (44.8 g, $180 \mathrm{mmol}, 1.0$ equiv) were suspended in $30 \%$ (v:v) aq. AcOH (350 mL). The mixture was vigorously stirred and refluxed for $5 \mathrm{~h}$. The reaction mixture was then diluted with cold water (250 $\mathrm{mL}$ ) and allowed to cool to rt, protecting it from light. After $1 \mathrm{~h}$, the crude product was collected by filtration, washed on the filter with ice water $(3 \times 150 \mathrm{~mL})$ and acetone $(3 \times 150 \mathrm{~mL})$, and air-dried in the dark overnight to afford 1-Hydroxy-1,2-benziodoxol-3-(1H)-one (15) (44.3 g, $168 \mathrm{mmol}, 93 \%)$ as a white solid.

${ }^{1} \mathrm{H}$ NMR $\left(400 \mathrm{MHz}\right.$, DMSO- $\left.d_{6}\right) \delta 8.02(\mathrm{dd}, J=7.7,1.4 \mathrm{~Hz}, 1 \mathrm{H}, \operatorname{Ar} H), 7.97(\mathrm{~m}, 1 \mathrm{H}, \operatorname{Ar} H), 7.85(\mathrm{dd}, J$ $=8.2,0.7 \mathrm{~Hz}, 1 \mathrm{H}, \mathrm{ArH}), 7.71(\mathrm{td}, J=7.6,1.2 \mathrm{~Hz}, 1 \mathrm{H}, \operatorname{ArH}) .{ }^{13} \mathrm{C}$ NMR $\left(100 \mathrm{MHz}, \mathrm{DMSO}-d_{6}\right) \delta 167.7$, $134.5,131.5,131.1,130.4,126.3,120.4$. Consistent with reported data. ${ }^{[1]}$

\section{Triisopropylsilyl trimethylsilylacetylene (17)}

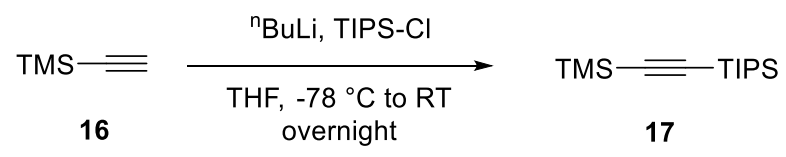

Following a reported procedure, ${ }^{[2]} n$-butyllithium ( $2.5 \mathrm{M}$ in hexanes, $28 \mathrm{~mL}, 70 \mathrm{mmol}, 0.98$ equiv) was added dropwise to a stirred solution of ethynyltrimethylsilane (16) $(7.0 \mathrm{~g}, 71 \mathrm{mmol}, 1.0$ equiv) in THF $(100 \mathrm{~mL})$ at $-78{ }^{\circ} \mathrm{C}$. The mixture was warmed to $0^{\circ} \mathrm{C}$ and stirred for $5 \mathrm{~min}$. The mixture was then cooled back to $-78^{\circ} \mathrm{C}$ and chlorotriisopropylsilane $(15 \mathrm{~mL}, 71 \mathrm{mmol}, 1.0$ equiv) was added dropwise. The mixture was then allowed to warm to room temperature and stirred overnight. A saturated solution of ammonium chloride $(100 \mathrm{~mL})$ was added, and the reaction mixture was extracted with diethyl ether $(2 \times 100 \mathrm{~mL})$. The combined organic layers were washed with water and brine, then dried over $\mathrm{MgSO}_{4}$, filtered and concentrated under reduced pressure to obtain a colorless liquid which was further purified by filtration on silica eluting with pentane $(500 \mathrm{~mL})$ to yield $17(16 \mathrm{~g}, 64$ mmol, $90 \%$ ) as a colorless liquid.

${ }^{1} \mathrm{H}$ NMR $\left(400 \mathrm{MHz}, \mathrm{CDCl}_{3}\right) \delta 1.08(\mathrm{~m}, 21 \mathrm{H}, \mathrm{TIPS}), 0.18$ (s, 9H, TMS). Consistent with reported data. $^{[2]}$

\section{1-[(Triiso-propylsilyl)ethynyl]-1,2-benziodoxol-3(1H)-one (TIPS-EBX, 1a)}

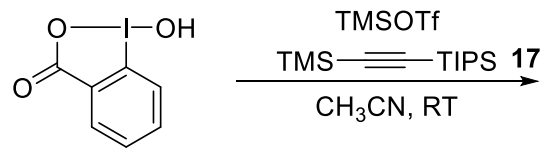

15<smiles>O=C1OI(C#C[In])c2ccccc21</smiles>

$1 \mathrm{a}$ 
Following a reported procedure, ${ }^{[3]}$ 2-iodosylbenzoic acid (15) $(8.0 \mathrm{~g}, 30 \mathrm{mmol}, 1.0$ equiv) was charged in an oven-dried round-bottomed $250 \mathrm{~mL}$ flask equipped with a magnetic stirrer. The solid was placed under a nitrogen atmosphere and anhydrous acetonitrile $(100 \mathrm{~mL})$ was added. The mixture was cooled to $0^{\circ} \mathrm{C}$. Trimethylsilyltriflate $(6.0 \mathrm{~mL}, 33 \mathrm{mmol}, 1.1$ equiv) was added dropwise. After $15 \mathrm{~min}$, (trimethylsilyl)(triisopropylsilyl)acetylene (17) $(8.5 \mathrm{~g}, 33 \mathrm{mmol}, 1.1$ equiv) was added dropwise. After $30 \mathrm{~min}$, the suspension became an orange solution. Pyridine $(2.7 \mathrm{~mL}, 33 \mathrm{mmol}, 1.1$ equiv) was added dropwise. After $15 \mathrm{~min}$, the reaction mixture was transferred in a one-neck 500 $\mathrm{mL}$ flask and concentrated under vacuum to afford a yellow solid. The solid was dissolved in DCM $(100 \mathrm{~mL})$ and transferred in a $500 \mathrm{~mL}$ separatory funnel. The organic layer was washed with a $1 \mathrm{M}$ $\mathrm{HCl}$ solution $(50 \mathrm{~mL})$ and the aqueous layer was extracted with DCM $(100 \mathrm{~mL})$. The organic layers were combined, washed with a saturated solution of $\mathrm{NaHCO}_{3}(2 \times 100 \mathrm{~mL})$, dried over $\mathrm{MgSO}_{4}$, filtered and the solvent was evaporated under reduced pressure. Recrystallization from acetonitrile (40 mL) afforded TIPS-EBX (3) (9.2 g, $21.5 \mathrm{mmol}, 71 \%)$ as colorless crystals.

$\mathrm{Mp}$ (Dec.) $170-176{ }^{\circ} \mathrm{C} .{ }^{1} \mathrm{H}$ NMR $(400 \mathrm{MHz}, \mathrm{CDCl} 3) \delta 8.44(\mathrm{~m}, 1 \mathrm{H}, \mathrm{ArH}), 8.29(\mathrm{~m}, 1 \mathrm{H}, \mathrm{ArH}), 7.77(\mathrm{~m}$, $2 \mathrm{H}, \operatorname{ArH}), 1.16(\mathrm{~m}, 21 \mathrm{H}, \mathrm{TIPS}) .{ }^{13} \mathrm{C}$ NMR $(100 \mathrm{MHz}, \mathrm{CDCl} 3) \delta 166.4,134.6,132.3,131.4,131.4$, 126.1, 115.6, 114.1, 64.6, 18.4, 11.1. IR v $2943(\mathrm{~m}), 2865(\mathrm{~m}), 1716(\mathrm{~m}), 1618(\mathrm{~m}), 1604(\mathrm{~s}), 1584$ $(\mathrm{m}), 1557(\mathrm{~m}), 1465(\mathrm{~m}), 1439(\mathrm{w}), 1349(\mathrm{~m}), 1291(\mathrm{~m}), 1270(\mathrm{w}), 1244(\mathrm{~m}), 1140(\mathrm{~m}), 1016(\mathrm{~m})$, $999(\mathrm{~m}), 883(\mathrm{~m}), 833(\mathrm{~m}), 742(\mathrm{~m}), 702(\mathrm{~s}), 636(\mathrm{~m})$. Consistent with reported data. ${ }^{[3]}$

\section{1-[Phenylethynyl]-1,2-benziodoxol-3(1H)-one (Ph-EBX, 1b)}<smiles>O=C1OI(O)c2ccccc21</smiles>

15

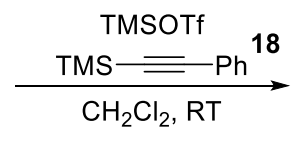

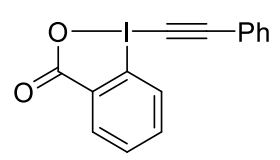

$1 \mathrm{~b}$

Following a reported procedure, ${ }^{[1]}$ trimethylsilyltriflate $(7.5 \mathrm{~mL}, 42 \mathrm{mmol}, 1.1$ equiv) was added dropwise to a suspension of 2-iodosylbenzoic acid (15) $\left(10 \mathrm{~g}, 38 \mathrm{mmol}, 1.0\right.$ equiv) in $\mathrm{CH}_{2} \mathrm{Cl}_{2}(100$ $\mathrm{mL})$ at $0{ }^{\circ} \mathrm{C}$. The mixture was stirred for $1 \mathrm{~h}$, followed by the dropwise addition of trimethyl(phenylethynyl)silane (18) $(8.2 \mathrm{~mL}, 42 \mathrm{mmol}, 1.1$ equiv) (slightly exothermic). The resulting suspension was stirred for $6 \mathrm{~h}$ at RT, during this time a white solid was formed. A saturated solution of $\mathrm{NaHCO}_{3}(100 \mathrm{~mL})$ was added and the mixture was stirred vigorously for $5 \mathrm{~min}$. The resulting suspension was filtered on a glass filter. The two layers of the mother liquors were separated and the organic layer was washed with sat. $\mathrm{NaHCO}_{3}(100 \mathrm{~mL})$, dried over $\mathrm{MgSO}_{4}$, filtered and evaporated under reduced pressure. The resulting mixture was combined with the solid obtained by filtration and boiled in $\mathrm{CH}_{3} \mathrm{CN}(150 \mathrm{~mL})$. The mixture was cooled down, filtered and dried under high vacuum to afford Ph-EBX (1b) (8.6 g, $25 \mathrm{mmol}, 65 \%)$ as a colorless solid.

Mp (Dec.) $155-160^{\circ} \mathrm{C} .{ }^{1} \mathrm{H}$ NMR $(400 \mathrm{MHz}$, Chloroform- $d) \delta 8.46(\mathrm{~m}, 1 \mathrm{H}, \operatorname{Ar} H), 8.28(\mathrm{~m}, 1 \mathrm{H}, \operatorname{Ar} H)$, $7.80(\mathrm{~m}, 2 \mathrm{H}, \mathrm{ArH}), 7.63(\mathrm{~m}, 2 \mathrm{H}, \mathrm{ArH}), 7.48(\mathrm{~m}, 3 \mathrm{H}, \mathrm{ArH}) .{ }^{13} \mathrm{C}$ NMR (101 MHz, Chloroform- $\left.d\right) \delta$ 163.9, 134.9, 132.9, 132.5, 131.6, 131.3. 130.8, 128.8, 126.2, 120.5, 116.2, 106.6, 50.2. Consistent with reported data. ${ }^{[1]}$

1-[4-Trifluoromethylphenylethynyl]-1,2-benziodoxol-3(1H)-one (1c)<smiles>O=C1OI(O)c2ccccc21</smiles>

15<smiles>CSC#Cc1ccc(C(F)(F)F)cc1</smiles>

19<smiles>O=C1OI(C#Cc2ccc(C(F)(F)F)cc2)c2ccccc21</smiles>

$1 c$ 
Following a reported procedure, ${ }^{[4]}$ trimethylsilyl triflate $(1.0 \mathrm{~mL}, 5.5 \mathrm{mmol}, 1.1$ equiv) was added to a suspension of 2-iodosylbenzoic acid (15) (1.3 g, 5.0 mmol, 1.0 equiv) in $\mathrm{CH}_{2} \mathrm{Cl}_{2}$ (15 mL) at RT. The resulting suspension was stirred for $1 \mathrm{~h}$, followed by the dropwise addition of trimethyl((4(trifluoromethyl)phenyl)ethynyl)silane (19) $(1.3 \mathrm{~mL}, 5.5 \mathrm{mmol}, 1.1$ equiv), which was dissolved in $\mathrm{CH}_{2} \mathrm{Cl}_{2}(1 \mathrm{~mL})$. The resulting suspension was stirred for $6 \mathrm{~h}$ at RT. A saturated solution of $\mathrm{NaHCO}_{3}$ $(20 \mathrm{~mL})$ was then added and the mixture was stirred vigorously for $30 \mathrm{~min}$, the two layers were separated and the organic layer was washed with sat. $\mathrm{NaHCO}_{3}(20 \mathrm{~mL})$, dried over $\mathrm{MgSO}_{4}$, filtered and evaporated under reduced pressure. The resulting solid was boiled in $\mathrm{CH}_{3} \mathrm{CN}(20 \mathrm{~mL})$. The mixture was cooled down, filtered and dried under high vacuum to afford 1c (1.3 g, $3.2 \mathrm{mmol}, 64 \%)$ as a pale yellow solid.

${ }^{1} \mathrm{H}$ NMR (400 MHz, Chloroform- $d$ ) $\delta 8.46-8.38(\mathrm{~m}, 1 \mathrm{H}, \mathrm{ArH}), 8.28-8.19(\mathrm{~m}, 1 \mathrm{H}, \mathrm{ArH}), 7.84-7.74$ $(\mathrm{m}, 2 \mathrm{H}, \mathrm{ArH}), 7.74-7.65(\mathrm{~m}, 4 \mathrm{H}, \mathrm{ArH}) .{ }^{13} \mathrm{C}$ NMR (101 MHz, Chloroform- $\left.d\right) \delta$ 166.6, 135.0, 133.0, 132.6, 132.2 (q, Jc-F = 33.0 Hz), 131.7, 131.2, 126.3, 125.7 (q, Jc-F = 3.6 Hz), 124.4, 123.4 (q, Jc-F $=272.6 \mathrm{~Hz}), 116.1,104.2,53.7$. Consistent with reported data. ${ }^{[4]}$

\section{1-[4-Bromophenylethynyl]-1,2-benziodoxol-3(1H)-one (1d)}<smiles>O=C1OI(O)c2ccccc21</smiles>

15

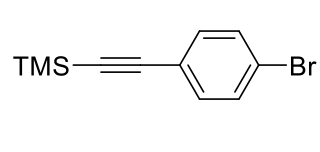

20

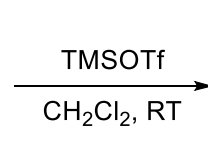

Following a reported procedure, ${ }^{[5]}$ trimethylsilyl triflate $(1.0 \mathrm{~mL}, 5.5 \mathrm{mmol}, 1.1$ equiv) was added to a suspension of 2-iodosylbenzoic acid (15) (1.3 g, 5.0 mmol, 1.0 equiv) in $\mathrm{CH}_{2} \mathrm{Cl}_{2}$ (15 mL) at RT. The resulting suspension was stirred for $1 \mathrm{~h}$, followed by the dropwise addition of ((4bromophenyl)ethynyl)trimethylsilane (20) $\left(1.2 \mathrm{~g}, 5.5 \mathrm{mmol}, 1.1\right.$ equiv), which was dissolved in $\mathrm{CH}_{2} \mathrm{Cl}_{2}$ $(1 \mathrm{~mL})$. The resulting suspension was stirred for $6 \mathrm{~h}$ at RT. A saturated solution of $\mathrm{NaHCO}_{3}(20 \mathrm{~mL})$ was then added and the mixture was stirred vigorously for $30 \mathrm{~min}$, the two layers were separated and the organic layer was washed with sat. $\mathrm{NaHCO}_{3}(20 \mathrm{~mL})$, dried over $\mathrm{MgSO}_{4}$, filtered and evaporated under reduced pressure. The resulting solid was boiled in $\mathrm{CH}_{3} \mathrm{CN}(20 \mathrm{~mL})$. The mixture was cooled down, filtered and dried under high vacuum to afford 1d (1.4 g, $3.3 \mathrm{mmol}, 66 \%)$ as a pale yellow solid.

Mp 158-163 ${ }^{\circ} \mathrm{C}$ (decomposition). ${ }^{1} \mathrm{H}$ NMR $(400 \mathrm{MHz}$, Chloroform- $d)$ ס $8.51-8.30(\mathrm{~m}, 1 \mathrm{H}, \mathrm{ArH}), 8.30$ - $8.13(\mathrm{~m}, 1 \mathrm{H}, \mathrm{ArH}), 7.84-7.72(\mathrm{~m}, 2 \mathrm{H}, \mathrm{ArH}), 7.58(\mathrm{~d}, 2 \mathrm{H}, J=8.5 \mathrm{~Hz}, \mathrm{ArH}), 7.46(\mathrm{~d}, 2 \mathrm{H}, J=8.5$ $\mathrm{Hz}, \operatorname{Ar} H) .{ }^{13} \mathrm{C}$ NMR $(101 \mathrm{MHz}$, Chloroform- $d) \delta 166.6,135.1,134.3,132.7,132.3,131.9,131.4$, $126.3,125.7,119.6,116.3,105.4,52.1$. Consistent with reported data. ${ }^{[6]}$

\section{1-((4-Formylphenyl)ethynyl)-1,2-benziodoxol-3(1H)-one (1e)}

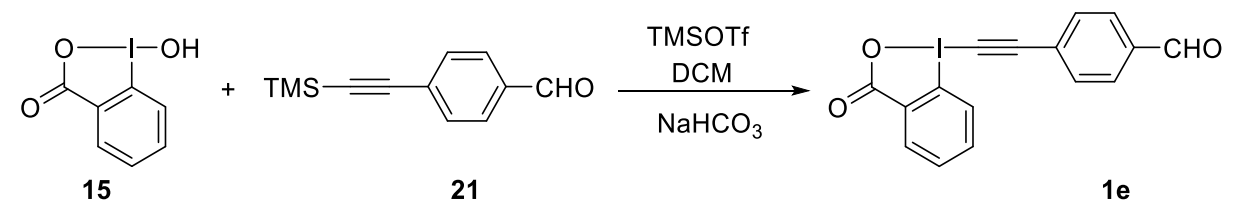

Following a reported procedure ${ }^{[7]}$ trimethylsilyl triflate $(0.89 \mathrm{~mL}, 4.9 \mathrm{mmol}, 1.1$ equiv) was added to a suspension of 2-iodosylbenzoic acid (15) (1.19 g, $4.49 \mathrm{mmol}, 1.00$ equiv) in $\mathrm{CH}_{2} \mathrm{Cl}_{2}(15 \mathrm{~mL})$ at RT. The resulting suspension was stirred for $1 \mathrm{~h}$, followed by the drop wise addition of ((4formylphenyl)ethynyl)trimethylsilane (21) $(1.00 \mathrm{~g}, 4.94 \mathrm{mmol}, 1.10$ equiv), which was dissolved in $\mathrm{CH}_{2} \mathrm{Cl}_{2}$ (1 mL). The resulting suspension was stirred for $6 \mathrm{~h}$ at RT. A saturated solution of $\mathrm{NaHCO}_{3}$ $(20 \mathrm{~mL})$ was then added and the mixture was stirred vigorously for 30 minutes, the two layers were separated and the organic layer was washed with sat. $\mathrm{NaHCO}_{3}(20 \mathrm{~mL})$, dried over $\mathrm{MgSO}_{4}$, filtered and evaporated under reduced pressure. The resulting solid was boiled in $\mathrm{CH}_{3} \mathrm{CN}(20 \mathrm{~mL})$. The 
mixture was cooled down, filtered and dried under high vacuum to afford $1 \mathrm{e}(0.80 \mathrm{~g}, 2.1 \mathrm{mmol}, 41 \%)$ as a yellow solid.

${ }^{1} \mathrm{H}$ NMR (400 MHz, DMSO- $\left.d_{6}\right) \delta 10.08(\mathrm{~s}, 1 \mathrm{H}, \mathrm{CHO}), 8.35(\mathrm{~d}, J=9.1 \mathrm{~Hz}, 1 \mathrm{H}, \mathrm{ArH}), 8.14(\mathrm{dd}, J=7.4$, $1.7 \mathrm{~Hz}, 1 \mathrm{H}, \mathrm{ArH}), 8.02(\mathrm{~d}, J=8.5 \mathrm{~Hz}, 2 \mathrm{H}, \mathrm{ArH}), 7.96-7.88(\mathrm{~m}, 3 \mathrm{H}, \mathrm{ArH}), 7.82(\mathrm{t}, J=7.3 \mathrm{~Hz}, 1 \mathrm{H}$, ArH). ${ }^{13} \mathrm{C}$ NMR $\left(101 \mathrm{MHz}\right.$, DMSO- $\left.d_{6}\right) \delta$ 192.6, 166.3, 136.7, 135.3, 133.2, 131.9, 131.4, 129.8, 127.7, 126.1, 116.4, 102.9, 56.6. Consistent with reported data. ${ }^{[7]}$

Trimethyl((4-cyanophenyl)ethynyl)silane (24)

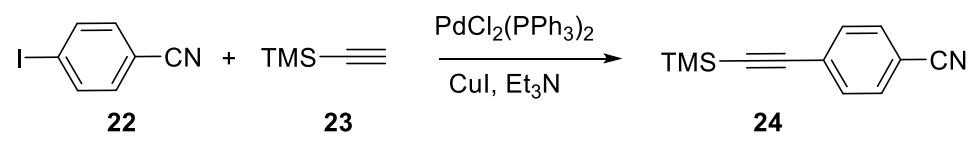

Following a slight modification of the reported procedure, ${ }^{[8]}$ 4-iodobenzonitrile (22) (1.00 g, 4.37 mmol, 1.0 equiv) was dissolved in triethylamine $(10 \mathrm{~mL})$ (without prior drying). After three freezethraw-pump cycles, $\mathrm{PdCl}_{2}\left(\mathrm{PPh}_{3}\right)_{2}(92 \mathrm{mg}, 0.13 \mathrm{mmol}, 3 \mathrm{~mol} \%)$ and copper iodide $(42 \mathrm{mg}, 0.22 \mathrm{mmol}$, $5 \mathrm{~mol} \%)$ were added under $\mathrm{N}_{2}$. After the addition of ethynyltrimethylsilane (23) $(1.2 \mathrm{~mL}, 8.7 \mathrm{mmol}$, 2.0 equiv) the green suspension was stirred at RT for $3 \mathrm{~h}$. The reaction mixture was concentrated under vacuum, dissolved in $\mathrm{CH}_{2} \mathrm{Cl}_{2}(30 \mathrm{~mL})$, washed with a saturated ammonium chloride solution $(30 \mathrm{~mL})$ and water $(30 \mathrm{~mL})$. The organic layers were then dried over $\mathrm{MgSO}_{4}$, filtered and concentrated under vacuum. The resulting oil was purified by column chromatography (pentane/ethyl acetate 25:1) to afford 4-((trimethylsilyl)ethynyl)benzonitrile (24) (847 mg, $4.25 \mathrm{mmol}$, $97 \%)$ as a white solid.

${ }^{1} \mathrm{H}$ NMR (400 MHz, Chloroform- $\left.d\right) \delta 7.59(\mathrm{~d}, J=8.7 \mathrm{~Hz}, 2 \mathrm{H}, \operatorname{Ar} H), 7.53(\mathrm{~d}, J=8.8 \mathrm{~Hz}, 2 \mathrm{H}, \mathrm{ArH}), 0.26$ (s, 9H, SiCH$\left.H_{3}\right) .{ }^{13} \mathrm{C}$ NMR (101 MHz, Chloroform-d) $\delta 132.6,132.1,128.1,118.6,111.9,103.1,99.7$, -0.12 . Consistent with reported data. ${ }^{[9]}$

\section{1-[4-Cyanophenylethynyl]-1,2-benziodoxol-3(1H)-one (1f)}<smiles>O=C1OI(O)c2ccccc21</smiles>

15

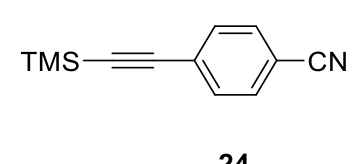

24
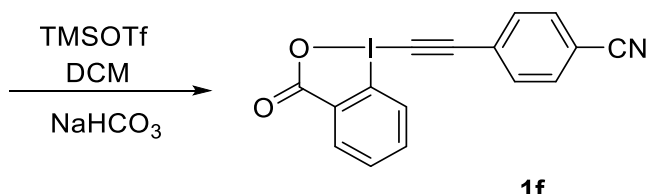

$1 f$

Following a reported procedure,$^{[5]}$ trimethylsilyl triflate $(0.73 \mathrm{~mL}, 4.0 \mathrm{mmol}, 1.1$ equiv) was added to a suspension of 2-iodosylbenzoic acid (15) $\left(963 \mathrm{mg}, 3.65 \mathrm{mmol}, 1.00\right.$ equiv) in $\mathrm{CH}_{2} \mathrm{Cl}_{2}$ (12 $\left.\mathrm{mL}\right)$ at RT. The resulting suspension was stirred for $1 \mathrm{~h}$, followed by the drop wise addition of ((4cyanophenyl)ethynyl)trimethylsilane (24) $(800 \mathrm{mg}, 4.01 \mathrm{mmol}, 1.10$ equiv), which was dissolved in $\mathrm{CH}_{2} \mathrm{Cl}_{2}(1 \mathrm{~mL})$. The resulting suspension was stirred for $6 \mathrm{~h}$ at RT. A saturated solution of $\mathrm{NaHCO}_{3}$ $(20 \mathrm{~mL})$ was then added and the mixture was stirred vigorously for 30 minutes, the two layers were separated and the organic layer was washed with sat. $\mathrm{NaHCO}_{3}(20 \mathrm{~mL})$, dried over $\mathrm{MgSO}_{4}$, filtered and evaporated under reduced pressure. The resulting solid was boiled in $\mathrm{CH}_{3} \mathrm{CN}(20 \mathrm{~mL})$. The mixture was cooled down, filtered and dried under high vacuum to afford 1f (865 mg, $2.32 \mathrm{mmol}$, $64 \%)$ as a pale brown solid.

${ }^{1} \mathrm{H}$ NMR (400 MHz, DMSO- $\left.d_{6}\right) \delta 8.34(\mathrm{~d}, J=8.2 \mathrm{~Hz}, 1 \mathrm{H}, \operatorname{Ar} H), 8.13(\mathrm{dd}, J=7.4,1.7 \mathrm{~Hz}, 1 \mathrm{H}, \operatorname{Ar} H$ ), $7.99(\mathrm{~d}, J=8.6 \mathrm{~Hz}, 2 \mathrm{H}, \operatorname{ArH}), 7.90(\mathrm{~d}, J=8.6 \mathrm{~Hz}, 3 \mathrm{H}, \operatorname{ArH}), 7.81(\mathrm{t}, J=7.3 \mathrm{~Hz}, 1 \mathrm{H}, \operatorname{ArH}) .{ }^{13} \mathrm{C}$ NMR $\left(101 \mathrm{MHz}, \mathrm{DMSO}-d_{6}\right) \delta 166.3,135.3,133.3,132.8,131.9,131.4,131.4,127.8,125.3,118.2,116.4$, $112.7,102.0,57.4$. Consistent with reported data. ${ }^{[5]}$ 


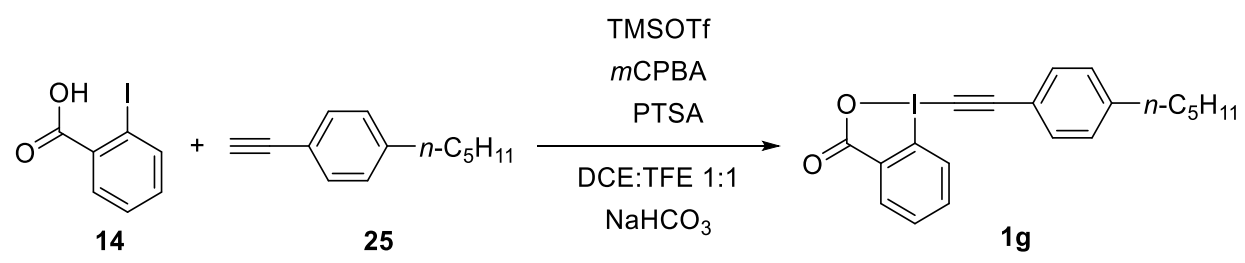

Following a reported procedure, ${ }^{[10]}$ in a sealed tube, 2-iodobenzoic acid (14) $(1.00 \mathrm{~g}, 4.03 \mathrm{mmol}$, 1.00 equiv), 4-methylbenzenesulfonic acid $(775 \mathrm{mg}, 4.03 \mathrm{mmol}, 1.00$ equiv) and mCPBA (994 mg, $4.44 \mathrm{mmol}, 1.10$ equiv) were suspended in DCE:TFE $1: 1(12 \mathrm{~mL})$ and stirred for $1 \mathrm{~h}$ at $55^{\circ} \mathrm{C}$. After $1 \mathrm{~h}, 1$-ethynyl-4-pentylbenzene $(25)(1.1 \mathrm{~mL}, 5.6 \mathrm{mmol}, 1.4$ equiv) was added and the reaction was stirred at $55^{\circ} \mathrm{C}$ for $24 \mathrm{~h}$. After $24 \mathrm{~h}$, the solvent was evaporated and the residue was redissolved in $\mathrm{CH}_{2} \mathrm{Cl}_{2}(20 \mathrm{~mL})$ and stirred vigorously with $\mathrm{NaHCO}_{3}$ sat. $(30 \mathrm{~mL})$. After $1 \mathrm{~h}$, the reaction mixture was transferred into a separating funnel and the layers were separated. The aqueous layer was extracted with $\mathrm{CH}_{2} \mathrm{Cl}_{2}(2 \times 50 \mathrm{~mL})$. The combined organic layers were washed with sat. $\mathrm{NaHCO}_{3}$, dried over $\mathrm{MgSO}_{4}$, filtered and concentrated under vacuum. The resulting solid was boiled in $\mathrm{MeCN}(20 \mathrm{~mL})$, then filtered and the collected solid was further purified by column chromatography using pure ethyl acetate. Trituration in pentane afforded $1 \mathrm{~g}(191 \mathrm{mg}, 0.457 \mathrm{mmol}, 11 \%)$ as a pale yellow solid.

${ }^{1} \mathrm{H}$ NMR (400 MHz, Chloroform- $\left.d\right) \delta 8.45-8.40(\mathrm{~m}, 1 \mathrm{H}, \mathrm{ArH}), 8.28-8.21(\mathrm{~m}, 1 \mathrm{H}, \mathrm{ArH}), 7.79-7.74$ $(\mathrm{m}, 2 \mathrm{H}, \mathrm{ArH}), 7.56-7.48(\mathrm{~m}, 2 \mathrm{H}, \mathrm{ArH}), 7.26-7.23(\mathrm{~m}, 2 \mathrm{H}, \mathrm{ArH}), 2.71-2.60(\mathrm{~m}, 2 \mathrm{H}, \mathrm{ArCH}), 1.69$ $-1.54\left(\mathrm{~m}, 2 \mathrm{H}, \mathrm{ArCH}_{2} \mathrm{CH}_{2}\right), 1.40-1.27\left(\mathrm{~m}, 4 \mathrm{H}, \mathrm{CH}_{2} \mathrm{CH}_{2} \mathrm{CH}_{3}\right), 0.90$ (t, $\left.J=6.8 \mathrm{~Hz}, 3 \mathrm{H}, \mathrm{CH}_{2} \mathrm{CH}_{3}\right) .{ }^{13} \mathrm{C}$ NMR (101 MHz, Chloroform-d) $\delta 166.6,146.7,135.0,133.0,132.6,131.7,131.5,129.0,126.3$, $117.7,116.4,107.4,49.4,36.2,31.5,31.0,22.6,14.1$. Consistent with reported data. ${ }^{[10]}$

\section{1-[3-Fluorophenylethynyl]-1,2-benziodoxol-3(1H)-one (1h)}<smiles>O=C1OI(O)c2ccccc21</smiles>

15

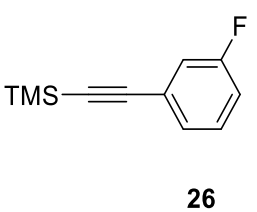

26

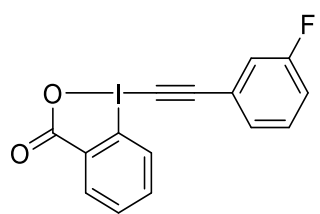

$1 \mathrm{~h}$

Following a reported procedure ${ }^{[10]}$ trimethylsilyl triflate $(1.0 \mathrm{~mL}, 5.5 \mathrm{mmol}, 1.1$ equiv) was added to a suspension of 2-iodosylbenzoic acid (15) (1.32 g, 5.00 mmol, 1.00 equiv) in $\mathrm{CH}_{2} \mathrm{Cl}_{2}(15 \mathrm{~mL})$ at RT. The resulting suspension was stirred for $1 \mathrm{~h}$, followed by the drop wise addition of ((3fluorophenyl)ethynyl)trimethylsilane (26) $(1.1 \mathrm{~mL}, 5.5 \mathrm{mmol}, 1.1$ equiv). The resulting suspension was stirred for $6 \mathrm{~h}$ at RT. A saturated solution of $\mathrm{NaHCO}_{3}(20 \mathrm{~mL})$ was then added and the mixture was stirred vigorously for 30 minutes, the two layers were separated and the organic layer was washed with sat. $\mathrm{NaHCO}_{3}(20 \mathrm{~mL})$, dried over $\mathrm{MgSO}_{4}$, filtered and evaporated under reduced pressure. The resulting solid was boiled in $\mathrm{CH}_{3} \mathrm{CN}(20 \mathrm{~mL})$. The mixture was cooled down, filtered and the collected solid was dried under high vacuum to afford $\mathbf{1 h}(787 \mathrm{mg}, 2.15 \mathrm{mmol}, 43 \%)$ as a colorless solid.

${ }^{1} \mathrm{H}$ NMR (400 MHz, DMSO- $\left.d_{6}\right) \delta 8.33(\mathrm{dd}, J=8.2,0.8 \mathrm{~Hz}, 1 \mathrm{H}, \mathrm{ArH}), 8.13(\mathrm{dd}, J=7.4,1.7 \mathrm{~Hz}, 1 \mathrm{H}$, ArH), 7.91 (ddd, $J=8.2,7.2,1.7 \mathrm{~Hz}, 1 \mathrm{H}, \mathrm{ArH}), 7.81(\mathrm{td}, J=7.3,0.9 \mathrm{~Hz}, 1 \mathrm{H}, \operatorname{ArH}), 7.64-7.59(\mathrm{~m}$, $1 \mathrm{H}, \mathrm{ArH}), 7.58-7.53(\mathrm{~m}, 2 \mathrm{H}, \mathrm{ArH}), 7.47-7.37(\mathrm{~m}, 1 \mathrm{H}, \mathrm{ArH}) .{ }^{13} \mathrm{C} N M R\left(101 \mathrm{MHz} \text {, DMSO- } d_{6}\right)^{1} 166.3$, $161.8(\mathrm{~d}, J=245.6 \mathrm{~Hz}), 135.3,131.9,131.3,131.2(\mathrm{~d}, J=8.7 \mathrm{~Hz}), 129.0(\mathrm{~d}, J=2.9 \mathrm{~Hz}), 127.7$, $122.4(\mathrm{~d}, J=9.6 \mathrm{~Hz}), 119.2(\mathrm{~d}, J=23.4 \mathrm{~Hz}), 118.1(\mathrm{~d}, J=21.1 \mathrm{~Hz}), 116.4,102.5(\mathrm{~d}, J=3.3 \mathrm{~Hz})$, 53.8. ${ }^{19} \mathrm{~F}$ NMR $\left(376 \mathrm{MHz}, \mathrm{DMSO}-d_{6}\right) \delta-111.7$. Consistent with reported data. ${ }^{[10]}$

${ }^{1}$ One carbon is not resolved. 


\section{1-[2-Bromophenylethynyl]-1,2-benziodoxol-3(1H)-one (1i)}
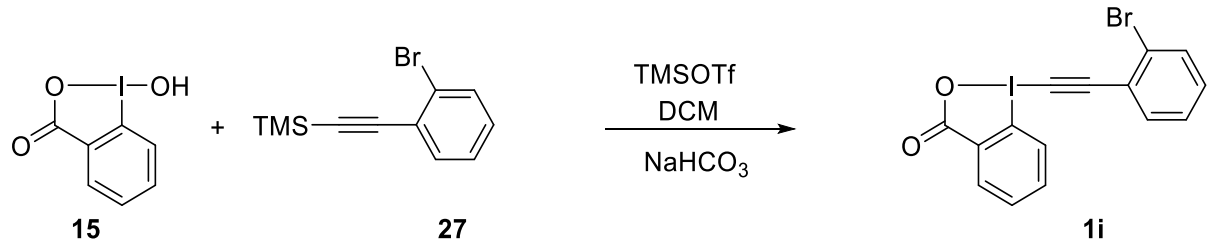

Following a reported procedure ${ }^{[6]}$ trimethylsilyl triflate $(1.0 \mathrm{~mL}, 5.5 \mathrm{mmol}, 1.1$ equiv) was added to a suspension of 2-iodosylbenzoic acid (15) $\left(1.32 \mathrm{~g}, 5.00 \mathrm{mmol}, 1.00\right.$ equiv) in $\mathrm{CH}_{2} \mathrm{Cl}_{2}(15 \mathrm{~mL})$ at RT. The resulting suspension was stirred for $3 \mathrm{~h}$, followed by the drop wise addition of ((2bromophenyl)ethynyl)trimethylsilane (27) $(1.17 \mathrm{~g}, 5.50 \mathrm{mmol}, 1.10$ equiv). The resulting suspension was stirred for $6 \mathrm{~h}$ at RT. A saturated solution of $\mathrm{NaHCO}_{3}(20 \mathrm{~mL})$ was then added and the mixture was stirred vigorously for 30 minutes, the two layers were separated and the organic layer was washed with sat. $\mathrm{NaHCO}_{3}(20 \mathrm{~mL})$, dried over $\mathrm{MgSO}_{4}$, filtered and evaporated under reduced pressure. The resulting solid was boiled in $\mathrm{CH}_{3} \mathrm{CN}(20 \mathrm{~mL})$. The mixture was cooled down, filtered and the collected solid was dried under high vacuum to afford $1 \mathrm{i}(1.50 \mathrm{~g}, 3.51 \mathrm{mmol}, 70 \%)$ as a colorless solid.

${ }^{1} \mathrm{H}$ NMR $(400 \mathrm{MHz}$, Chloroform- $d) \delta 8.44(\mathrm{td}, J=7.3,2.1 \mathrm{~Hz}, 2 \mathrm{H}, \operatorname{Ar} H), 7.84-7.74(\mathrm{~m}, 2 \mathrm{H}, \operatorname{Ar} H)$, $7.68(\mathrm{~d}, J=1.1 \mathrm{~Hz}, 1 \mathrm{H}, \operatorname{ArH}), 7.61(\mathrm{dd}, J=7.6,1.7 \mathrm{~Hz}, 1 \mathrm{H}, \operatorname{ArH}), 7.36(\mathrm{~m}, 2 \mathrm{H}, \operatorname{ArH}) .{ }^{13} \mathrm{C}$ NMR $(101$ $\mathrm{MHz}$, Chloroform-d) ${ }^{1} \delta$ 166.6, 135.2, 134.7, 133.0, 132.7 , 131.8, 131.3, 127.6, 126.8, 126.4, 123.2, $116.5,104.3,55.4$. Consistent with reported data. ${ }^{[6]}$

\section{3-Bromopropyl 4-((trimethylsilyl)ethynyl)benzoate (29)}

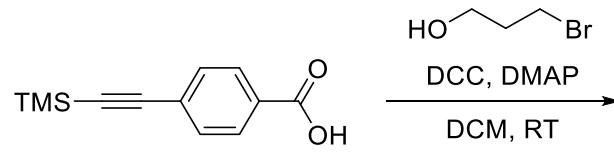

28

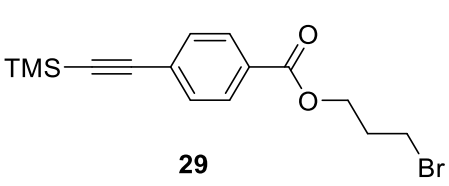

29

4-(Dimethylamino)-pyridine ( $67 \mathrm{mg}, 0.55 \mathrm{mmol}, 12 \mathrm{~mol} \%$ ) was added to a stirred reaction mixture of 4-((trimethylsilyl)ethynyl)benzoic acid (28) $(1.0 \mathrm{~g}, 4.6 \mathrm{mmol}, 1.0$ equiv), dicyclohexylcarbodiimide (1.0 g, 5.0 mmol, 1.1 equiv), 3-bromopropan-1-ol $\left(0.62 \mathrm{~mL}, 6.9 \mathrm{mmol}, 1.5\right.$ equiv) in dry $\mathrm{CH}_{2} \mathrm{Cl}_{2}$ (15 $\mathrm{mL}$ ) at room temperature. The reaction mixture was filtered after $15 \mathrm{~h}$ and the solid was rinsed with dichloromethane $(2 \times 10 \mathrm{~mL})$. The combined filtrates were concentrated under vacuum. Purification by column chromatography pentane/ethyl acetate 9:1 afforded 3-bromopropyl 4((trimethylsilyl)ethynyl)benzoate (29) $(1.3 \mathrm{~g}, 3.8 \mathrm{mmol}, 82 \%)$ as a white solid.

Mp 37.2 - 39. ${ }^{\circ} \mathrm{C}$. Rf (pentane/ethyl acetate 9:1): 0.8. ${ }^{1} \mathrm{H}$ NMR $(400 \mathrm{MHz}$, Chloroform- $d$ ) $\delta 7.96$ (d, $\left.J=8.6 \mathrm{~Hz}, 2 \mathrm{H}, \operatorname{Ar} H), 7.52(\mathrm{~d}, J=8.7 \mathrm{~Hz}, 2 \mathrm{H}, \operatorname{ArH}), 4.46(\mathrm{t}, J=6.0 \mathrm{~Hz}, 2 \mathrm{H}, \mathrm{OCH})_{2}\right), 3.54(\mathrm{t}, J=6.6$ $\left.\mathrm{Hz}, 2 \mathrm{H}, \mathrm{BrCH}_{2}\right), 2.32\left(\mathrm{p}, J=6.4 \mathrm{~Hz}, 2 \mathrm{H}, \mathrm{CH}_{2} \mathrm{CH}_{2}\right), 0.26$ (s, 9H, TMS). ${ }^{13} \mathrm{C}$ NMR $(101 \mathrm{MHz}$, Chloroform- $d) \delta 165.8,131.9,129.5,129.4,127.9,104.0,97.9,62.9,31.8,29.4,-0.2$. IR $\left(\mathrm{v}_{\max }, \mathrm{cm}^{-}\right.$ 1) $2957(w), 2926(w), 2353(w), 2162(w), 1725(s), 1608(w), 1410(w), 1269(s), 1176(m), 1108$ (m), $1016(\mathrm{w}), 868$ (s), $769(\mathrm{~m})$. HRMS (ESI/QTOF) m/z: [M + Na] ${ }^{+}$Calcd for $\mathrm{C}_{15} \mathrm{H}_{19}{ }^{79} \mathrm{BrNaO}_{2} \mathrm{Si}^{+}$ 361.0230, 363.0209; Found 361.0235, 363.0216.

3-Azidopropyl 4-((trimethylsilyl)ethynyl)benzoate (30)
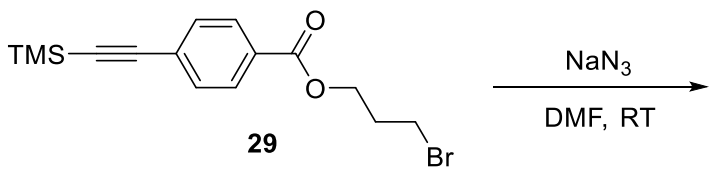

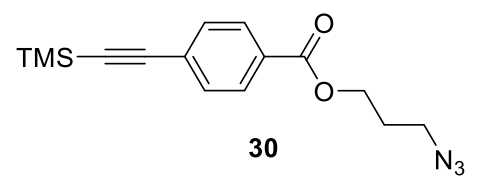


$\mathrm{NaN}_{3}(0.25 \mathrm{~g}, 3.9 \mathrm{mmol}, 1.2$ equiv) was added to a solution of 3-bromopropyl 4((trimethylsilyl)ethynyl)benzoate (29) $(1.1 \mathrm{~g}, 3.2 \mathrm{mmol}, 1.0$ equiv) in dry DMF (16 mL) at RT. The mixture was heated at $50^{\circ} \mathrm{C}$ overnight. Ice water was added and the aqueous layer was extracted with diethyl ether (3x). The combined organic layers were washed with brine (3x), dried over $\mathrm{MgSO}_{4}$ and concentrated under vacuum. The crude was purified by column chromatography pentane to pentane/ethyl acetate 95:5 to afford 3-azidopropyl 4-((trimethylsilyl)ethynyl)benzoate (30) (0.60 g, $2.0 \mathrm{mmol}, 61 \%$ yield) as a pale yellow oil.

Rf: 0.7 (pentane/ethyl acetate 9:1). ${ }^{1} \mathrm{H}$ NMR $(400 \mathrm{MHz}$, Chloroform- $d) \delta 7.96(\mathrm{~d}, J=8.7 \mathrm{~Hz}, 2 \mathrm{H}, \operatorname{Ar} H)$, $7.52\left(\mathrm{~d}, J=8.7 \mathrm{~Hz}, 2 \mathrm{H}, \operatorname{Ar} H\right.$ ), $4.41\left(\mathrm{t}, J=6.2 \mathrm{~Hz}, 2 \mathrm{H}, \mathrm{OCH}_{2}\right), 3.48\left(\mathrm{t}, J=6.7 \mathrm{~Hz}, 2 \mathrm{H}, \mathrm{N}_{3} \mathrm{CH}_{2}\right.$ ), 2.05 (p, J = 6.5 Hz, 2H, $\mathrm{CH}_{2} \mathrm{CH}_{2}$ ), 0.26 (s, 9H, TMS). ${ }^{13} \mathrm{C}$ NMR (101 MHz, Chloroform-d) $\delta$ 165.8, 131.9, 129.5, 129.4, 128.0, 104.0, 97.9, 62.0, 48.3, 28.2, -0.2. IR $\left(\mathrm{v}_{\max }, \mathrm{cm}^{-1}\right) 3666(\mathrm{w}), 2970(\mathrm{~m}), 2902(\mathrm{~m})$, 2101 (s), 1725 (s), 1608 (m), $1472(\mathrm{w}), 1410(\mathrm{w}), 1281(\mathrm{~s}), 1115(\mathrm{~m}), 1065(\mathrm{~m}), 868(\mathrm{~s}), 856(\mathrm{~s}), 769$ (m). HRMS (ESI/QTOF) m/z: [M + Na] ${ }^{+}$Calcd for $\mathrm{C}_{15} \mathrm{H}_{19} \mathrm{~N}_{3} \mathrm{NaO}_{2} \mathrm{Si}^{+}$324.1139; Found 324.1137.

\section{1-((4-(Prop-2-yn-1-yl-benzoate)ethynyl-1,2-benziodoxol-3(1H)-one (1j)}

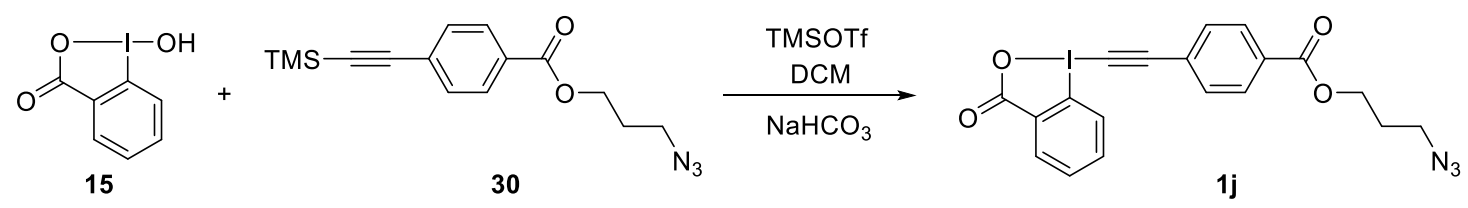

Trimethylsilyl triflate $(0.34 \mathrm{~mL}, 1.9 \mathrm{mmol}, 1.1$ equiv) was added to a suspension of 2-iodosylbenzoic acid (15) (454 mg, $1.72 \mathrm{mmol}, 1.00$ equiv) in $\mathrm{CH}_{2} \mathrm{Cl}_{2}(5 \mathrm{~mL})$ at $\mathrm{RT}$. The resulting suspension was stirred for $1 \mathrm{~h}$, followed by the drop wise addition of 3-azidopropyl 4-((trimethylsilyl)ethynyl)benzoate (30) $\left(570 \mathrm{mg}, 1.89 \mathrm{mmol}, 1.10\right.$ equiv), which was dissolved in $\mathrm{CH}_{2} \mathrm{Cl}_{2}(0.5 \mathrm{~mL})$. The resulting suspension was stirred for $4 \mathrm{~h}$ at RT. A saturated solution of $\mathrm{NaHCO}_{3}(10 \mathrm{~mL})$ was then added and the mixture was stirred vigorously for 30 minutes, the two layers were separated and the organic layer was washed with sat. $\mathrm{NaHCO}_{3}(10 \mathrm{~mL})$, dried over $\mathrm{MgSO}_{4}$, filtered and evaporated under reduced pressure. The resulting solid was boiled in $\mathrm{CH}_{3} \mathrm{CN}(10 \mathrm{~mL})$. The mixture was cooled down, filtered and dried under high vacuum to afford $1 \mathbf{j}$ (431 $\mathrm{mg}, 0.907 \mathrm{mmol}, 53 \%)$ as a pale brown solid.

Mp (Dec.) $106.4-109.3{ }^{\circ} \mathrm{C} .{ }^{1} \mathrm{H}$ NMR $(400 \mathrm{MHz}$, Chloroform- $d) \delta 8.47-8.41(\mathrm{~m}, 1 \mathrm{H}, \mathrm{ArH}), 8.34-$ $8.20(\mathrm{~m}, 1 \mathrm{H}, \mathrm{ArH}), 8.10(\mathrm{~d}, J=8.7 \mathrm{~Hz}, 2 \mathrm{H}, \operatorname{ArH}), 7.87-7.76(\mathrm{~m}, 2 \mathrm{H}, \operatorname{Ar} H), 7.67(\mathrm{~d}, J=8.6 \mathrm{~Hz}, 2 \mathrm{H}$, $\operatorname{ArH}$ ), $4.46\left(\mathrm{t}, J=6.2 \mathrm{~Hz}, 2 \mathrm{H}, \mathrm{OCH}_{2}\right), 3.50\left(\mathrm{t}, J=6.6 \mathrm{~Hz}, 2 \mathrm{H}, \mathrm{N}_{3} \mathrm{CH}_{2}\right), 2.08\left(\mathrm{p}, J=6.4 \mathrm{~Hz}, 2 \mathrm{H}, \mathrm{CH}_{2} \mathrm{CH}_{2}\right)$. ${ }^{13} \mathrm{C}$ NMR (101 MHz, Chloroform-d) $\delta 166.4,165.3,135.1,132.7,132.6,131.8,131.6,131.2,129.8$, 126.2, 125.1, 116.1, 105.0, 62.4, 54.1, 48.2, 28.2. IR $\left(\mathrm{v}_{\max }, \mathrm{cm}^{-1}\right) 3678(\mathrm{w}), 3389(\mathrm{w}), 2988(\mathrm{~s}), 2908$ (s), $2366(\mathrm{w}), 2101(\mathrm{~m}), 1774(\mathrm{w}), 1719(\mathrm{~m}), 1638(\mathrm{~m}), 1454(\mathrm{w}), 1398(\mathrm{~m}), 1281(\mathrm{~m}), 1071(\mathrm{~s}), 868$ (m), $769(\mathrm{~m})$. HRMS (ESI/QTOF) m/z: $[\mathrm{M}+\mathrm{H}]^{+}$Calcd for $\mathrm{C}_{19} \mathrm{H}_{15} \mathrm{IN}_{3} \mathrm{O}_{4}+476.0102$; Found 476.0112.

\section{Prop-2-yn-1-yl 4-((trimethylsilyl)ethynyl)benzoate (32)}

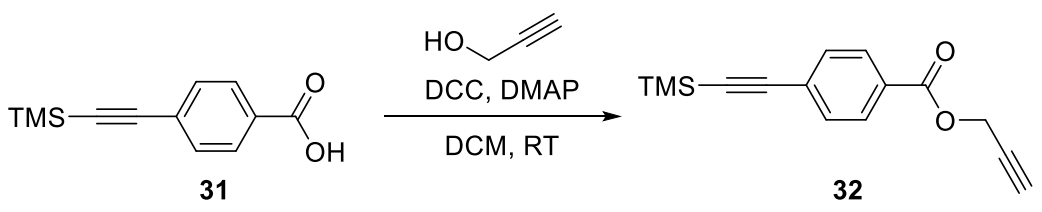

4-(Dimethylamino)-pyridine ( $27 \mathrm{mg}, 0.22 \mathrm{mmol}, 12 \mathrm{~mol} \%$ ) was added to a stirred reaction mixture of 4-((trimethylsilyl)ethynyl)benzoic acid (31) $(0.40 \mathrm{~g}, 1.8 \mathrm{mmol}, 1.0$ equiv), dicyclohexylcarbodiimide (0.42 g, $2.0 \mathrm{mmol}, 1.1$ equiv) and 2-propynol $\left(0.16 \mathrm{~mL}, 2.8 \mathrm{mmol}, 1.5\right.$ equiv) in dry $\mathrm{CH}_{2} \mathrm{Cl}_{2}(7 \mathrm{~mL})$ at $\mathrm{RT}$. The reaction mixture was filtered after $15 \mathrm{~h}$ and the solid was rinsed with dichloromethane $(2 \mathrm{x}$ $5 \mathrm{~mL}$ ). The combined filtrates were concentrated under vacuum. Purification by column 
chromatography pentane/ethyl acetate 9:1 afforded prop-2-yn-1-yl 4((trimethylsilyl)ethynyl)benzoate (32) (375 mg, $1.46 \mathrm{mmol}, 80 \%$ ) as a white solid.

Mp $58-60{ }^{\circ} \mathrm{C} . \mathrm{Rf}$ (pentane/ethyl acetate 9:1): 0.8. ${ }^{1} \mathrm{H}$ NMR $(400 \mathrm{MHz}$, Chloroform- $d) \delta 8.00(\mathrm{~d}, J=$ $8.7 \mathrm{~Hz}, 2 \mathrm{H}, \operatorname{ArH}), 7.52(\mathrm{~d}, J=8.7 \mathrm{~Hz}, 2 \mathrm{H}, \operatorname{ArH}), 4.92(\mathrm{~d}, J=2.5 \mathrm{~Hz}, 2 \mathrm{H}, \mathrm{OCH}$ ), 2.52 (t, $J=2.5 \mathrm{~Hz}$, $1 \mathrm{H}, \mathrm{CH}), 0.26$ (s, 9H, TMS). ${ }^{13} \mathrm{C}$ NMR $(101 \mathrm{MHz}$, Chloroform-d) $\delta 165.2,131.9,129.6,128.9,128.2$, 103.9, 98.1, 77.5, 75.1, 52.6, -0.2. IR $\left(\mathrm{v}_{\max }, \mathrm{cm}^{-1}\right) 3678(\mathrm{w}), 3296(\mathrm{w}), 2963(\mathrm{~m}), 2902(\mathrm{~m}), 2359(\mathrm{w})$, $2162(\mathrm{w}), 2125(\mathrm{w}), 1725(\mathrm{~s}), 1608(\mathrm{~m}), 1410(\mathrm{~m}), 1373(\mathrm{~m}), 1262(\mathrm{~s}), 1176(\mathrm{~m}), 1102(\mathrm{~s}), 868(\mathrm{~s})$, $763(\mathrm{~m})$. HRMS (APCI/QTOF) m/z: [M + H] ${ }^{+}$Calcd for $\mathrm{C}_{15} \mathrm{H}_{17} \mathrm{O}_{2} \mathrm{Si}^{+} 257.0992$; Found 257.0995.

\section{1-((4-(Prop-2-yn-1-yl-benzoate)ethynyl-1,2-benziodoxol-3(1H)-one (1k)}

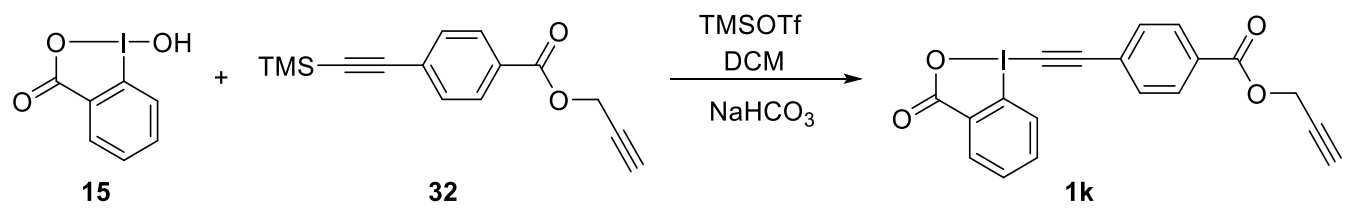

Trimethylsilyl triflate $(0.99 \mathrm{~mL}, 5.5 \mathrm{mmol}, 1.1$ equiv) was added to a suspension of 2-iodosylbenzoic acid (15) (1.32 g, $5.50 \mathrm{mmol}, 1.0$ equiv) in $\mathrm{CH}_{2} \mathrm{Cl}_{2}(12 \mathrm{~mL})$ at $\mathrm{RT}$. The resulting suspension was stirred for $1 \mathrm{~h}$, followed by the drop wise addition of prop-2-yn-1-yl 4-((trimethylsilyl)ethynyl)benzoate (32) $\left(1.41 \mathrm{~g}, 5.50 \mathrm{mmol}, 1.1\right.$ equiv), which was dissolved in $\mathrm{CH}_{2} \mathrm{Cl}_{2}(1 \mathrm{~mL})$. The resulting suspension was stirred for $4 \mathrm{~h}$ at RT. A saturated solution of $\mathrm{NaHCO}_{3}(20 \mathrm{~mL})$ was then added and the mixture was stirred vigorously for 30 minutes, the two layers were separated and the organic layer was washed with sat. $\mathrm{NaHCO}_{3}(20 \mathrm{~mL})$, dried over $\mathrm{MgSO}_{4}$, filtered and evaporated under reduced pressure. The resulting solid was purified by column chromatography with pure ethyl acetate to afford 1k (174 mg, $0.404 \mathrm{mmol}, 8 \%$ ) as a white crystalline solid.

Mp (Dec.) $107-118^{\circ} \mathrm{C} .{ }^{1} \mathrm{H}$ NMR $(400 \mathrm{MHz}$, Chloroform- $d) \delta 8.44(\mathrm{dd}, J=6.3,2.8 \mathrm{~Hz}, 1 \mathrm{H}, \operatorname{Ar} H)$, $8.28-8.21(\mathrm{~m}, 1 \mathrm{H}, \operatorname{ArH}), 8.13(\mathrm{~d}, J=8.4 \mathrm{~Hz}, 2 \mathrm{H}, \operatorname{ArH}), 7.88-7.74(\mathrm{~m}, 2 \mathrm{H}, \operatorname{ArH}), 7.68(\mathrm{~d}, J=8.5$ $\mathrm{Hz}, 2 \mathrm{H}, \mathrm{ArH}), 4.96\left(\mathrm{~d}, J=2.5 \mathrm{~Hz}, 2 \mathrm{H}, \mathrm{OCH} \mathrm{H}_{2}\right), 2.55(\mathrm{t}, J=2.5 \mathrm{~Hz}, 1 \mathrm{H}, \mathrm{CH}) .{ }^{13} \mathrm{C}$ NMR $(101 \mathrm{MHz}$, Chloroform-d) $\delta 166.5,164.7,135.1,132.8,132.7,132.6,131.8,131.2,131.0,130.0,126.3,125.4$, 116.1, 104.9, 75.4, 54.2, 52.9. IR ( $\left.\mathrm{V}_{\max }, \mathrm{cm}^{-1}\right) 3278(\mathrm{w}), 2988(\mathrm{~m}), 2908(\mathrm{~m}), 2353(\mathrm{w}), 2119(\mathrm{w})$, $1725(\mathrm{~s}), 1608(\mathrm{~m}), 1577(\mathrm{w}), 1417(\mathrm{~m}), 1380(\mathrm{~m}), 1262(\mathrm{~s}), 1102(\mathrm{~s}), 1053(\mathrm{~m}), 1016(\mathrm{~m}), 967(\mathrm{w})$, $868(\mathrm{w}), 868(\mathrm{w}), 739(\mathrm{~m})$. HRMS (ESI/QTOF) m/z: $[\mathrm{M}+\mathrm{H}]^{+}$Calcd for $\mathrm{C}_{19} \mathrm{H}_{12} \mathrm{IO}_{4}{ }^{+}$430.9775; Found 430.9781 .

\section{Ph-VBX (34)}<smiles>O=C(O)c1ccccc1I</smiles>

14

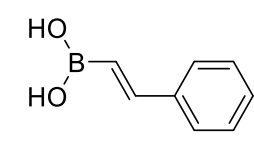

33

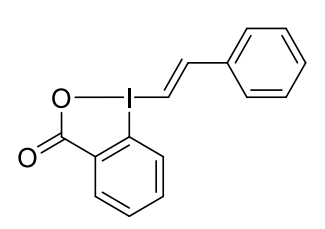

34

Following a reported procedure ${ }^{[11]} \mathrm{mCPBA}(1.25 \mathrm{~g}, 5.50 \mathrm{mmol}, 1.10$ equiv) was added to a solution of 2-iodobenzoic acid (14) (1.25 g, $5.00 \mathrm{mmol}, 1.00$ equiv) in $\mathrm{CH}_{2} \mathrm{Cl}_{2}$ (25 mL). The reaction mixture was cooled to $0{ }^{\circ} \mathrm{C}$ and trifluoromethanesulfonic acid $(0.66 \mathrm{~mL}, 7.5 \mathrm{mmol}, 1.5$ equiv) was added at this temperature before being stirred at r.t for $15 \mathrm{~min}$. The reaction mixture was again cooled to $0 \stackrel{\circ}{ } \mathrm{C}$ over $10 \mathrm{~min}$ and $(E)$-styrylboronic acid (33) $(1.0 \mathrm{~g}, 7.0 \mathrm{mmol}, 1.4$ equiv) and the mixture was stirred at $\mathrm{r} . \mathrm{t}$ for $1 \mathrm{~h}$. A saturated $\mathrm{NaHCO}_{3}$ solution $(25 \mathrm{~mL})$ was added and the mixture was stirred for $1 \mathrm{~h}$. The mixture was diluted with $\mathrm{CH}_{2} \mathrm{Cl}_{2}(25 \mathrm{~mL})$ and $\mathrm{H}_{2} \mathrm{O}(25 \mathrm{~mL})$ and the layers were separated. The aqueous layer was extracted with $\mathrm{CH}_{2} \mathrm{Cl}_{2}(3 \times 50 \mathrm{~mL})$ and the combined organic layers were washed with brine, dried $\left(\mathrm{Mg}_{2} \mathrm{SO}_{4}\right)$ and filtered. The solvent was removed under reduced pressure. The crude 
mixture contained a lot of impurities, so purification by column chromatography over silica gel (DCM with 2 to $10 \%$ Acetone) afforded $500 \mathrm{mg}$ of pure Ph-VBX 34 as off white crystalline solid (29\%).

${ }^{1} \mathrm{H}$ NMR $\left(400 \mathrm{MHz}\right.$, Methanol- $\left.d_{4}\right) \delta 8.28(\mathrm{dd}, J=5.8,3.4 \mathrm{~Hz}, 1 \mathrm{H}, \mathrm{ArH}), 7.96(\mathrm{~d}, J=15.4 \mathrm{~Hz}, 1 \mathrm{H}$, $\operatorname{ArH}), 7.74(\mathrm{dd}, J=5.9,3.5 \mathrm{~Hz}, 1 \mathrm{H}, \operatorname{ArH}), 7.70(\mathrm{dd}, J=7.5,3.5 \mathrm{~Hz}, 4 \mathrm{H}, \operatorname{Ar} H), 7.66(\mathrm{~d}, J=8.7 \mathrm{~Hz}, 1 \mathrm{H}$, $\operatorname{ArH}), 7.49(\mathrm{dd}, J=5.1,2.0 \mathrm{~Hz}, 3 \mathrm{H}, \operatorname{Ar} H)$. The NMR shifts match the literature data. ${ }^{[11]}$ 


\section{Library of organic dyes}

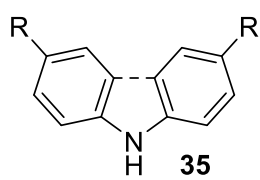

(6 equiv)

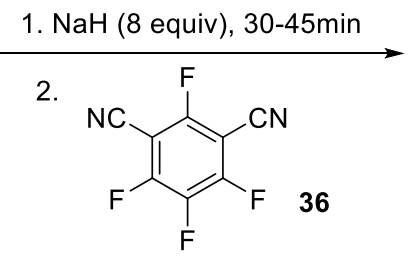

Solvent, $15 \mathrm{~h}$

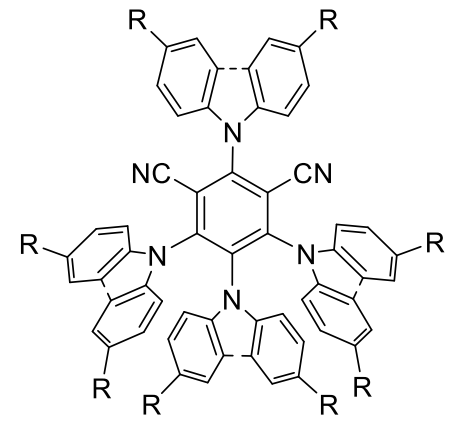

\section{General procedure 1:}

Sodium hydride (60\% suspension in mineral oil, 8.0 equiv) was added slowly to a stirred solution of substituted-carbazole 35a-d (5.0 equiv) in dry THF $(0.05 \mathrm{M})$ under a nitrogen atmosphere at RT. After 30 min, 2,4,5,6-tetrafluoroisophthalonitrile 36 (1.0 mmol, 1.0 equiv) was added. After stirring at $\mathrm{RT}$ for $15 \mathrm{~h}, 2 \mathrm{~mL}$ water was added to the reaction mixture to quench the excess of $\mathrm{NaH}$. The resulting mixture was then concentrated under reduced pressure. The crude product was purified by recrystallization from hexane $/ \mathrm{CH}_{2} \mathrm{Cl}_{2}$ then filtered. The brown liquid filtrate was concentrated and recrystallized as before. The combined solids were then purified by column chromatography on silica gel with DCM/Hexane.

\section{General procedure 2:}

Sodium hydride (60\% suspension in mineral oil, 8.0 equiv) was added slowly to a stirred solution of substituted-diphenylamine $35 \mathbf{d}-\mathbf{f}$ (6.0 equiv) in dry DMF $(0.1 \mathrm{M})$ under a nitrogen atmosphere at RT. After 45 min - 1 h, 2,4,5,6-tetrafluoroisophthalonitrile 36 (1.0 equiv) was added. After stirring at RT for $15 \mathrm{~h}$, water and ice were added to the reaction mixture to quench the excess of $\mathrm{NaH}$. The precipitate was filtered and purified by recrystallization from pentane/ $\mathrm{CH}_{2} \mathrm{Cl}_{2}$ then filtered. The brown liquid filtrate was concentrated and recrystallized as before. The combined solids were then purified by column chromatography on silica gel with DCM/Hexane.

\section{2,4,5,6-Tetra(9H-carbazol-9-yl)isophthalonitrile (4CzIPN, 2a)}

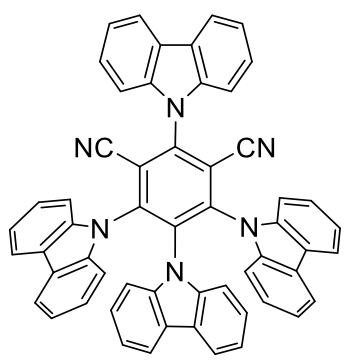

Following the general procedure 1 and starting from $9 \mathrm{H}$-carbazole $35 \mathrm{a}(1.67 \mathrm{~g}, 10.0 \mathrm{mmol}, 5.00$ equiv), sodium hydride $(0.60 \mathrm{~g}, 15 \mathrm{mmol}, 7.5$ equiv) and 2,4,5,6-tetrafluoroisophthalonitrile 36 ( 0.40 $\mathrm{g}, 2.0 \mathrm{mmol}$ ) in $40 \mathrm{~mL}$ of THF. Recrystallization (Hexanes $/ \mathrm{CH}_{2} \mathrm{Cl}_{2}(1: 1,90 \mathrm{~mL}$ )) afforded the crude product as a yellow powder. Column chromatography afforded 2,4,5,6-tetra(9H-carbazol-9yl)isophthalonitrile (2a) as a bright yellow crystalline solid (1.14 g, $1.45 \mathrm{mmol}, 73 \%$ yield).

$\mathrm{Rf}\left(\right.$ Hexane/DCM 1/1) $=0.29$. (yellow spot on TLC). ${ }^{1} \mathrm{H}$ NMR $(400 \mathrm{MHz}$, Chloroform- $d) \delta 8.2(\mathrm{~d}, J=$ $7.7 \mathrm{~Hz}, 2 \mathrm{H}, \operatorname{ArH}), 7.8-7.6(\mathrm{~m}, 8 \mathrm{H}, \operatorname{ArH}), 7.5$ (ddd, $J=8.0,6.6,1.6 \mathrm{~Hz}, 2 \mathrm{H}, \operatorname{ArH}), 7.3(\mathrm{~d}, J=7.5 \mathrm{~Hz}$, $2 \mathrm{H}, \operatorname{ArH}), 7.2(\mathrm{dd}, J=8.4,1.5 \mathrm{~Hz}, 4 \mathrm{H}, \operatorname{ArH}), 7.2-7.0(\mathrm{~m}, 8 \mathrm{H}, \operatorname{ArH}), 6.8(\mathrm{t}, J=7.8 \mathrm{~Hz}, 4 \mathrm{H}, \operatorname{ArH}), 6.6$ $(\mathrm{td}, J=7.6,1.2 \mathrm{~Hz}, 2 \mathrm{H}, \mathrm{ArH}) .{ }^{13} \mathrm{C}$ NMR $(101 \mathrm{MHz}$, Chloroform-d) $\delta 145.2,144.6,140.0,138.2,136.9$, 134.7, 127.0, 125.8, 124.9, 124.7, 124.5, 123.8, 122.4, 121.9, 121.4, 121.0, 120.4, 119.6, 116.3, $111.6,109.9,109.5,109.4 .{ }^{1} \mathrm{H}$ NMR shift in Chloroform- $d$ are consistent with reported data. ${ }^{[12]}$ 


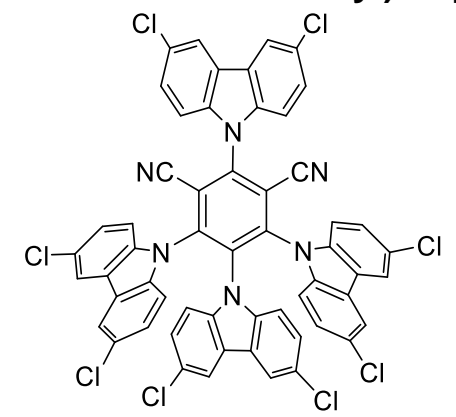

Following the general procedure 1 and starting from 3,6-dichloro-9H-carbazole 35b $(1.96 \mathrm{~g}, 6.00$ mmol, 6.0 equiv), sodium hydride $(320 \mathrm{mg}, 8.00 \mathrm{mmol}, 8.0$ equiv) and 2,4,5,6tetrafluoroisophthalonitrile $36(200 \mathrm{mg}, 1.00 \mathrm{mmol})$ in $20 \mathrm{~mL}$ of THF. Recrystallization (Hexanes $\left./ \mathrm{CH}_{2} \mathrm{Cl}_{2}(1: 2,80 \mathrm{~mL})\right)$ gave $900 \mathrm{mg}$ of yellow powder, then second recrystallization gave $325 \mathrm{mg}$ of brown powder. Column chromatography of the combined solid afforded $(2 \mathrm{r}, 4 \mathrm{~s}, 5 \mathrm{r})-2,4,5,6$ tetrakis(3,6-dichloro-9H-carbazol-9-yl)isophthalonitrile $\mathbf{( 2 b )}$ as a bright yellow crystalline solid (830 $\mathrm{mg}, 0.780 \mathrm{mmol}, 87 \%$ yield).

Rf (Hexane/DCM 1:1): 0.25. (yellow spot on TLC). ${ }^{1} \mathrm{H}$ NMR (400 MHz, DMSO- $\left.d_{6}\right) \delta 8.60(\mathrm{~d}, J=2.1$ $\mathrm{Hz}, 2 \mathrm{H}, \mathrm{ArH}), 8.15(\mathrm{~d}, J=2.1 \mathrm{~Hz}, 4 \mathrm{H}, \operatorname{Ar} H), 8.08(\mathrm{~d}, J=8.8 \mathrm{~Hz}, 2 \mathrm{H}, \operatorname{ArH}), 7.87$ (dd, $J=8.8,2.1 \mathrm{~Hz}$, $2 \mathrm{H}, \operatorname{Ar} H), 7.80(\mathrm{~d}, J=2.2 \mathrm{~Hz}, 2 \mathrm{H}, \operatorname{ArH}), 7.69(\mathrm{~d}, J=8.8 \mathrm{~Hz}, 4 \mathrm{H}, \operatorname{ArH}), 7.46(\mathrm{~d}, J=8.8 \mathrm{~Hz}, 2 \mathrm{H}, \operatorname{Ar} H)$, $7.32(\mathrm{dd}, J=8.8,2.2 \mathrm{~Hz}, 4 \mathrm{H}, \mathrm{ArH}), 6.93(\mathrm{dd}, J=8.8,2.2 \mathrm{~Hz}, 2 \mathrm{H}, \operatorname{ArH}) .{ }^{13} \mathrm{C}$ NMR $(101 \mathrm{MHz}$, DMSO$\left.d_{6}\right) \delta 145.0,144.5,138.5,137.4,136.5,135.8,134.5,127.8,127.0,126.4,125.7,125.3,124.2$, 123.8, 123.3, 121.6, 120.9, 120.3, 116.8, 112.6, 112.5, 112.3, 111.7. HRMS (ESI) calcd for $\mathrm{C}_{56} \mathrm{H}_{24} \mathrm{Cl}_{8} \mathrm{~N}_{6}[\mathrm{M}+]$ 1059.9565; found 1059.9573.

${ }^{1} \mathrm{H}$ NMR shift in $\mathrm{CDCl}_{3}$ are consistent with reported data. ${ }^{[13]}$ However, for better solubility and better resolution new ${ }^{1} \mathrm{H}$ and ${ }^{13} \mathrm{C}$ spectra were recorded in DMSO- $d_{6}$.

\section{(2r,4s,5r)-2,4,5,6-Tetrakis(3,6-di-tert-butyl-9H-carbazol-9-yl)isophthalonitrile (4tBuCzIPN, 2c)}

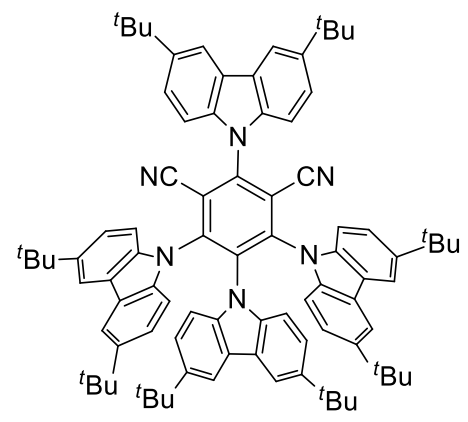

Following the general procedure 1 and starting from 3,6-tert-butyl-9H-carbazole $35 \mathrm{c}(1.22 \mathrm{~g}, 4.37$ mmol, 5.0 equiv), sodium hydride $(280 \mathrm{mg}, 7.00 \mathrm{mmol}, 8.0$ equiv) and 2,4,5,6tetrafluoroisophthalonitrile $36(175 \mathrm{mg}, 0.875 \mathrm{mmol})$ in $17.5 \mathrm{~mL}$ of THF, $(2 \mathrm{r}, 4 \mathrm{~s}, 5 \mathrm{r})-2,4,5,6-$ tetrakis(3,6-di-tert-butyl-9H-carbazol-9-yl)isophthalonitrile (2c) was obtained as a yellow (light orange) crystalline solid (940 $\mathrm{mg}, 0.759 \mathrm{mmol}, 87 \%$ yield) after recrystallization and column chromatography using dichloromethane:pentane (1:2).

${ }^{1} \mathrm{H}$ NMR $\left(400 \mathrm{MHz}\right.$, Acetone- $\left.d_{6}\right) \delta 8.42(\mathrm{~d}, J=1.8 \mathrm{~Hz}, 2 \mathrm{H}, \operatorname{Ar} H), 7.95(\mathrm{~d}, J=8.5 \mathrm{~Hz}, 2 \mathrm{H}, \mathrm{ArH}), 7.87$ $(\mathrm{d}, J=1.9 \mathrm{~Hz}, 4 \mathrm{H}, \mathrm{ArH}), 7.77(\mathrm{dd}, J=8.6,2.0 \mathrm{~Hz}, 2 \mathrm{H}, \mathrm{ArH}), 7.45(\mathrm{~d}, J=8.6 \mathrm{~Hz}, 4 \mathrm{H}, \mathrm{ArH}), 7.42(\mathrm{~d}, J$ $=1.9 \mathrm{~Hz}, 2 \mathrm{H}, \mathrm{ArH}), 7.14(\mathrm{dd}, J=8.7,2.0 \mathrm{~Hz}, 4 \mathrm{H}, \operatorname{ArH}), 7.10(\mathrm{~d}, J=8.6 \mathrm{~Hz}, 2 \mathrm{H}, \operatorname{ArH}), 6.65(\mathrm{dd}, J=$ 8.7, $2.0 \mathrm{~Hz}, 2 \mathrm{H}, \operatorname{ArH}), 1.50(\mathrm{~s}, 18 \mathrm{H}, t B u), 1.32(\mathrm{~s}, 36 \mathrm{H}, t B u), 1.24(\mathrm{~s}, 18 \mathrm{H}, t B u) .{ }^{13} \mathrm{C}$ NMR $(101 \mathrm{MHz}$, Acetone- $\left.d_{6}\right)^{2} \delta 146.0,144.9,144.6,144.1,143.1,139.0,137.6,136.5,136.2,124.6,124.3,124.2$,

2 The signals were not fully resolved. 
122.9, 121.8, 117.3, 116.8, 116.3, 115.3, 112.6, 110.4, 110.2, 109.8, 34.6, 34.3, 34.0, 31.4, 31.2. The characterization data matches the reference. ${ }^{[14]}$

\section{3,6-Dimethoxy-9H-carbazole (37)}<smiles>COc1ccc2[nH]c3ccc(OC)cc3c2c1</smiles>

Following a reported procedure, ${ }^{[15]}$ in a $25 \mathrm{~mL}$ microwave tube were placed 3,6-dibromo-9Hcarbazole ( $975 \mathrm{mg}, 3.00 \mathrm{mmol}, 1.0$ equiv) and copper(I) iodide (1.14 g, $6.00 \mathrm{mmol}, 2.0$ equiv) under a nitrogen atmosphere. DMF $(6 \mathrm{~mL})$ and a $30 \%$ solution of $\mathrm{NaOMe}$ in $\mathrm{MeOH}(4 \mathrm{~mL})$ were added. The tube was sealed and heated at $85^{\circ} \mathrm{C}$ for $15 \mathrm{~h}$, then at $105^{\circ} \mathrm{C}$ for $5 \mathrm{~h}$. The crude mixture was diluted in ethyl acetate and filtered over a silica pad, then concentrated under vacuum. The crude was purified by column chromatography with pentane/dichloromethane 1:1 to afford 3,6-dimethoxy9H-carbazole (37) (477 mg, $2.10 \mathrm{mmol}, 70.0 \%$ yield) as a white solid.

Rf (pentane/DCM 1:1): 0.6. ${ }^{1} \mathrm{H}$ NMR (400 MHz, Chloroform- $d$ ) $\delta 7.74$ (bs, $\left.1 \mathrm{H}, \mathrm{NH}\right), 7.51$ (d, J= 2.5 $\mathrm{Hz}, 2 \mathrm{H}, \operatorname{ArH}), 7.29$ (d, J = 8.8 Hz, 2H, ArH), 7.06 (dd, J=8.8, 2.5 Hz, 2H, ArH), 3.94 (s, 6H, OMe). ${ }^{13} \mathrm{C}$ NMR $(101 \mathrm{MHz}$, Chloroform-d) $\delta 153.6,135.2,123.7,115.2,111.5,102.8,56.0$. Consistent with reported data. ${ }^{[15]}$

(2r,4s,5r)-2,4,5,6-Tetrakis(3,6-dimethoxy-9H-carbazol-9-yl)isophthalonitrile (4MeOCzIPN, 2d)

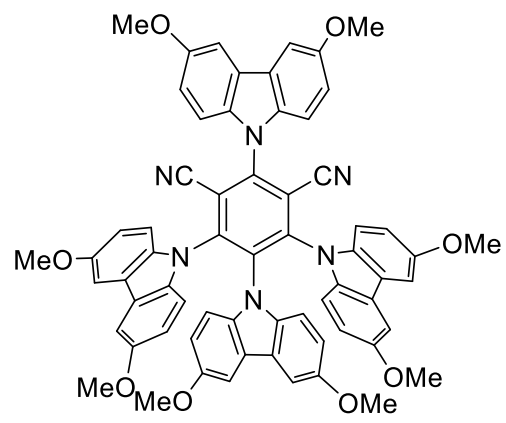

Following the general procedure 1 and starting from 3,6-dimethoxy-9H-carbazole 35d (443 mg, 1.95 mmol, 6.0 equiv), sodium hydride $(104 \mathrm{mg}, 2.60 \mathrm{mmol}, 8$ equiv) and 2,4,5,6tetrafluoroisophthalonitrile $36(65.0 \mathrm{mg}, 0.325 \mathrm{mmol})$ in $6.5 \mathrm{~mL}$ of THF. Recrystallization and column chromatography in pure dichloromethane afforded $(2 r, 4 s, 5 r)-2,4,5,6$-Tetrakis(3,6-dimethoxy- $9 \mathrm{H}$ carbazol-9-yl)isophthalonitrile (2d) as a red crystalline solid (321 mg, $0.312 \mathrm{mmol}, 94 \%$ yield).

${ }^{1} \mathrm{H}$ NMR (400 MHz, Chloroform- $d$ ) $\delta 7.48(\mathrm{~d}, J=2.5 \mathrm{~Hz}, 2 \mathrm{H}, \operatorname{ArH}), 7.41(\mathrm{~d}, J=8.8 \mathrm{~Hz}, 2 \mathrm{H}, \operatorname{ArH}), 7.18$ - $7.15(\mathrm{~m}, 2 \mathrm{H}, \operatorname{ArH}), 7.04(\mathrm{~d}, J=2.5 \mathrm{~Hz}, 4 \mathrm{H}, \operatorname{ArH}), 6.93(\mathrm{~d}, J=8.9 \mathrm{~Hz}, 4 \mathrm{H}, \operatorname{ArH}), 6.71(\mathrm{~d}, J=2.5 \mathrm{~Hz}$, $2 \mathrm{H}, \mathrm{ArH}), 6.64-6.53(\mathrm{~m}, 6 \mathrm{H}, \mathrm{ArH}), 6.19$ (dd, $J=8.9,2.5 \mathrm{~Hz}, 2 \mathrm{H}, \mathrm{ArH}), 3.87$ (s, 6H, OMe), 3.67 (s, $12 \mathrm{H}, \mathrm{OMe}), 3.57(\mathrm{~s}, 6 \mathrm{H}, \mathrm{OMe}) .{ }^{13} \mathrm{C}$ NMR $(101 \mathrm{MHz}$, Chloroform-d) $\delta 155.6,155.1,154.3,145.0$, 144.5, 135.3, 133.7, 133.5, 132.4, 125.6, 125.2, 124.5, 115.7, 114.9, 114.4, 113.6, 112.0, 110.8, $110.5,110.1,104.0,103.4,102.8,56.0,55.8,55.7$. The characterization data matches the reference. ${ }^{[16]}$ 


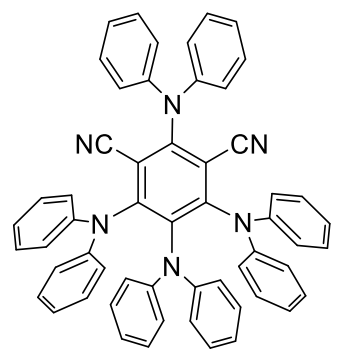

Following the general procedure 2 and starting from diphenylamine $35 \mathrm{e}(1.01 \mathrm{~g}, 6.00 \mathrm{mmol}, 6.0$ equiv), sodium hydride (320 mg, $8.00 \mathrm{mmol}, 8.0$ equiv) and 2,4,5,6-tetrafluoroisophthalonitrile 36 (200 mg, $1.00 \mathrm{mmol}$ ) in $10 \mathrm{~mL}$ of DMF. The deprotonation was performed at $50^{\circ} \mathrm{C}$ for $1 \mathrm{~h}$, followed by stirring at the same temperature for $4 \mathrm{~h}$. Recrystallization $\left(\mathrm{CH}_{2} \mathrm{Cl}_{2} /\right.$ pentane (1:2)) gave 2,4,5,6tetrakis(diphenylamino)isophthalonitrile (3a) as a yellow-orange crystalline solid (400 mg, 0.502 $\mathrm{mmol}, 50 \%$ yield).

Rf (pentane/DCM 1:1): 0.3. (yellow spot on TLC). ${ }^{1} \mathrm{H}$ NMR (400 MHz, Chloroform-d) $\delta 7.32-7.22$ $(\mathrm{m}, 4 \mathrm{H}, \mathrm{ArH}), 7.12-7.05(\mathrm{~m}, 12 \mathrm{H}, \mathrm{ArH}), 7.07-6.98(\mathrm{~m}, 2 \mathrm{H}, \mathrm{ArH}), 6.96-6.84(\mathrm{~m}, 8 \mathrm{H}, \mathrm{ArH}), 6.73-$ $6.63(\mathrm{~m}, 10 \mathrm{H}, \mathrm{ArH}), 6.56(\mathrm{~d}, J=7.4 \mathrm{~Hz}, 4 \mathrm{H}, \operatorname{Ar} H) .{ }^{13} \mathrm{C}$ NMR $(101 \mathrm{MHz}$, Chloroform- $d) \delta$ 154.2, 151.7, 145.5, 144.6, 143.1, 140.3, 129.4, 128.6, 127.5, 124.2, 123.9, 122.9, 122.6, 122.6, 121.1, 113.1, 113.0. IR ( $\left.v_{\max }, \mathrm{cm}^{-1}\right) 3065(\mathrm{w}), 3040(\mathrm{w}), 2361(\mathrm{w}), 1586(\mathrm{~m}), 1535(\mathrm{~m}), 1497(\mathrm{~s}), 1415(\mathrm{~s}), 1275$ (m), $1244(\mathrm{~m}), 1028(\mathrm{w}), 907(\mathrm{~m}), 742$ (s), 698 (s). HRMS (ESI/QTOF) m/z: [M + H] Calcd for $\mathrm{C}_{56} \mathrm{H}_{41} \mathrm{~N}_{6}{ }^{+}$797.3387; Found 797.3375.

The data were not in alignment with the reported values, ${ }^{[17]}$ a crystal structure confirmed the structure (see section 5).

\section{Bis(4-chlorophenyl)amine (38)}<smiles>Clc1ccc(Nc2ccc(Cl)cc2)cc1</smiles>

Following a reported procedure, ${ }^{[18]}$ in a $25 \mathrm{~mL}$ dry microwave vial was placed $\mathrm{Pd}_{2} \mathrm{dba}_{3}(27.5 \mathrm{mg}$, $0.0300 \mathrm{mmol}, 1 \mathrm{~mol} \%)$, BrettPhos (48.3 mg, $0.0900 \mathrm{mmol}, 3 \mathrm{~mol} \%)$, 1-chloro-4-iodobenzene (715 $\mathrm{mg}, 3.00 \mathrm{mmol}, 1$ equiv), 4-chloroaniline ( $459 \mathrm{mg}, 3.60 \mathrm{mmol}, 1.2$ equiv), $\mathrm{Cs}_{2} \mathrm{CO}_{3}$ (2.34 g, $7.20 \mathrm{mmol}$, 2.4 equiv). The vial was placed under an argon atmosphere and toluene $(6 \mathrm{~mL})$ was added. The reaction mixture was stirred at $70^{\circ} \mathrm{C}$ overnight and then at $95^{\circ} \mathrm{C}$ for $4 \mathrm{~h}$. The mixture was diluted with ethyl acetate, filtered over a celite pad and concentrated under vacuum. The crude was purified by column chromatography pentane/ethyl acetate 100:0 to $95: 5$ to afford bis(4-chlorophenyl)amine (38) (595 mg, $2.50 \mathrm{mmol}, 83 \%$ yield) as a yellow-brown solid.

Rf (pentane/ethyl acetate 95:5): 0.45. ${ }^{1} \mathrm{H}$ NMR (400 MHz, Chloroform- $d$ ) $\delta 7.25-7.17(\mathrm{~m}, 4 \mathrm{H}, \mathrm{ArH})$, $7.01-6.95(\mathrm{~m}, 4 \mathrm{H}, \mathrm{ArH}), 5.71$ (s, $1 \mathrm{H}, \mathrm{NH}) .{ }^{13} \mathrm{C}$ NMR (101 MHz, Chloroform-d) $\delta$ 141.3, 129.4, 126.2, 119.2. Consistent with reported data. ${ }^{[18]}$ 


\section{2,4,5,6-Tetrakis(bis(4-chlorophenyl)amino)isophthalonitrile (4CIDPAIPN, 3b)}

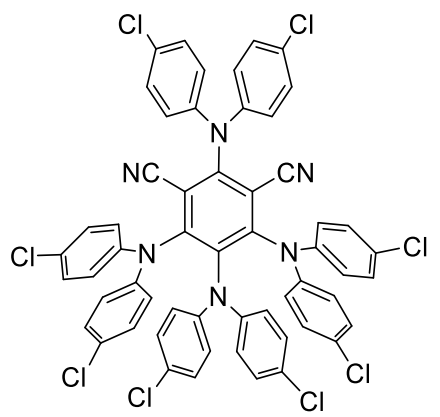

Following the general procedure 2, and starting from bis(4-chlorophenyl)amine $35 \mathbf{f}$ (550 $\mathrm{mg}, 2.31$ mmol, 6.0 equiv), sodium hydride $(123 \mathrm{mg}, 3.08 \mathrm{mmol}, 8.0$ equiv) and 2,4,5,6tetrafluoroisophthalonitrile $36(77.0 \mathrm{mg}, 0.385 \mathrm{mmol})$ in $4 \mathrm{~mL}$ of DMF. Recrystallization (Hexanes $/ \mathrm{CH}_{2} \mathrm{Cl}_{2}$ (1:1)) followed by column chromatography afforded 2,4,5,6-tetrakis(bis(4chlorophenyl)amino)isophthalonitrile (3b) as a bright yellow crystalline solid (332 $\mathrm{mg}, 0.310 \mathrm{mmol}$, $80 \%$ yield).

Rf (pentane/DCM 1:1): 0.57. (yellow spot on TLC). ${ }^{1} \mathrm{H}$ NMR (400 MHz, Chloroform- $d$ ) $\delta 7.28-7.23$ $(\mathrm{m}, 4 \mathrm{H}, \mathrm{ArH}), 7.13-7.07(\mathrm{~m}, 8 \mathrm{H}, \mathrm{ArH}), 6.98-6.91(\mathrm{~m}, 8 \mathrm{H}, \mathrm{ArH}), 6.59-6.54(\mathrm{~m}, 8 \mathrm{H}, \mathrm{ArH}), 6.46-$ $6.40(\mathrm{~m}, 4 \mathrm{H}, \mathrm{ArH}) .{ }^{13} \mathrm{C}$ NMR (101 MHz, Chloroform- $\left.d\right)^{3} \delta$ 153.2, 143.5, 142.8, 141.3, 138.8, 130.5, 130.2, 129.9, 129.2, 128.6, 128.3, 124.2, 123.6, 121.9, 112.5, 112.4. IR $\left(\mathrm{V}_{\max }, \mathrm{cm}^{-1}\right) 2367(\mathrm{w}), 1586$ (w), $1535(\mathrm{w}), 1485(\mathrm{~s}), 1409(\mathrm{w}), 1282(\mathrm{~m}), 1098(\mathrm{~m}), 1015(\mathrm{w}), 913(\mathrm{w}), 825(\mathrm{~m}), 729(\mathrm{~m}), 660(\mathrm{w})$. HRMS (APPI/LTQ-Orbitrap) m/z: [M+H] ${ }^{+}$Calcd for $\mathrm{C}_{56} \mathrm{H}_{33} \mathrm{Cl}_{8} \mathrm{~N}_{6}{ }^{+}$1069.0269; Found 1069.0277. A crystal structure confirmed the structure (see section 5 ).

\section{2,4,5,6-Tetrakis(bis(4-bromophenyl)amino)isophthalonitrile (4BrDPAIPN, 3c)}

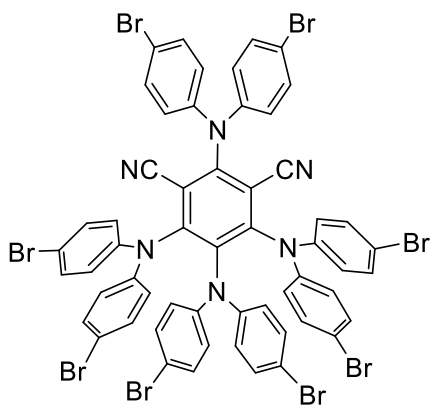

Following the general procedure 1 and starting from bis(4-bromophenyl)amine $\mathbf{3 5 g}(1.10 \mathrm{~g}, 4.47$ mmol, 5.00 equiv), sodium hydride $(0.286 \mathrm{~g}, 7.16 \mathrm{mmol}, 8.00$ equiv) and 2,4,5,6tetrafluoroisophthalonitrile $36(0.179 \mathrm{~g}, 0.895 \mathrm{mmol})$ in $18 \mathrm{~mL}$ of THF. Before recrystallization, water $(5 \mathrm{~mL})$ was added and the mixture was sonicated for $5 \mathrm{~min}$ before being filtered. Two recrystallization (pentane $/ \mathrm{CH}_{2} \mathrm{Cl}_{2}$ 2:1) afforded 2,4,5,6-tetrakis(bis(4-bromophenyl)amino)isophthalonitrile (3c) as a bright yellow crystalline solid (1.34 g, $0.938 \mathrm{mmol}, 94 \%$ yield).

Rf (pentane/DCM 1:1): 0.6. (yellow spot on TLC). ${ }^{1} \mathrm{H}$ NMR (400 MHz, Chloroform- $d$ ) $\delta 7.45-7.37$ $(\mathrm{m}, 4 \mathrm{H}, \mathrm{ArH}), 7.26-7.22(\mathrm{~m}, 8 \mathrm{H}, \mathrm{ArH}), 7.13-7.05(\mathrm{~m}, 4 \mathrm{H}, \mathrm{ArH}), 6.93-6.82(\mathrm{~m}, 4 \mathrm{H}, \mathrm{ArH}), 6.58-$ $6.45(\mathrm{~m}, 8 \mathrm{H}, \mathrm{ArH}), 6.42-6.33(\mathrm{~m}, 4 \mathrm{H}, \mathrm{ArH}) .{ }^{13} \mathrm{C}$ NMR $(101 \mathrm{MHz}$, Chloroform-d) $\delta 153.1,151.4$, 143.9, 143.2, 141.7, 138.7, 132.9, 132.3, 131.3, 124.6, 124.0, 122.2, 118.3, 118.0, 116.2, 112.6, 112.5. IR $\left(\mathrm{V}_{\max }, \mathrm{cm}^{-1}\right) 2367(\mathrm{w}), 1777(\mathrm{w}), 1586(\mathrm{w}), 1523(\mathrm{w}), 1485(\mathrm{~s}), 1421(\mathrm{~m}), 1389(\mathrm{~m}), 1307$

${ }^{3}$ One carbon is not resolved. 
(m), $1282(\mathrm{~m}), 1231(\mathrm{w}), 1072(\mathrm{~m}), 1009(\mathrm{~m}), 818(\mathrm{~m}), 710(\mathrm{w})$. HRMS (ESI/QTOF) m/z: [M + $\mathrm{K}]^{+}$Calcd for $\mathrm{C}_{56} \mathrm{H}_{32} \mathrm{Br}_{8} \mathrm{KN}_{6}{ }^{+}$1458.5787; Found 1458.5812 . 


\section{Physical measurements}

\subsection{Spectroscopic characterization}

4CZIPN and 4DPAIPN derivatives 2 and 3 were studied at 10 umol.L-1 in acetonitrile. Absorbance was recorded between 200 and $800 \mathrm{~nm}$. The excitation wavelength for fluorescence was $360 \mathrm{~nm}$.

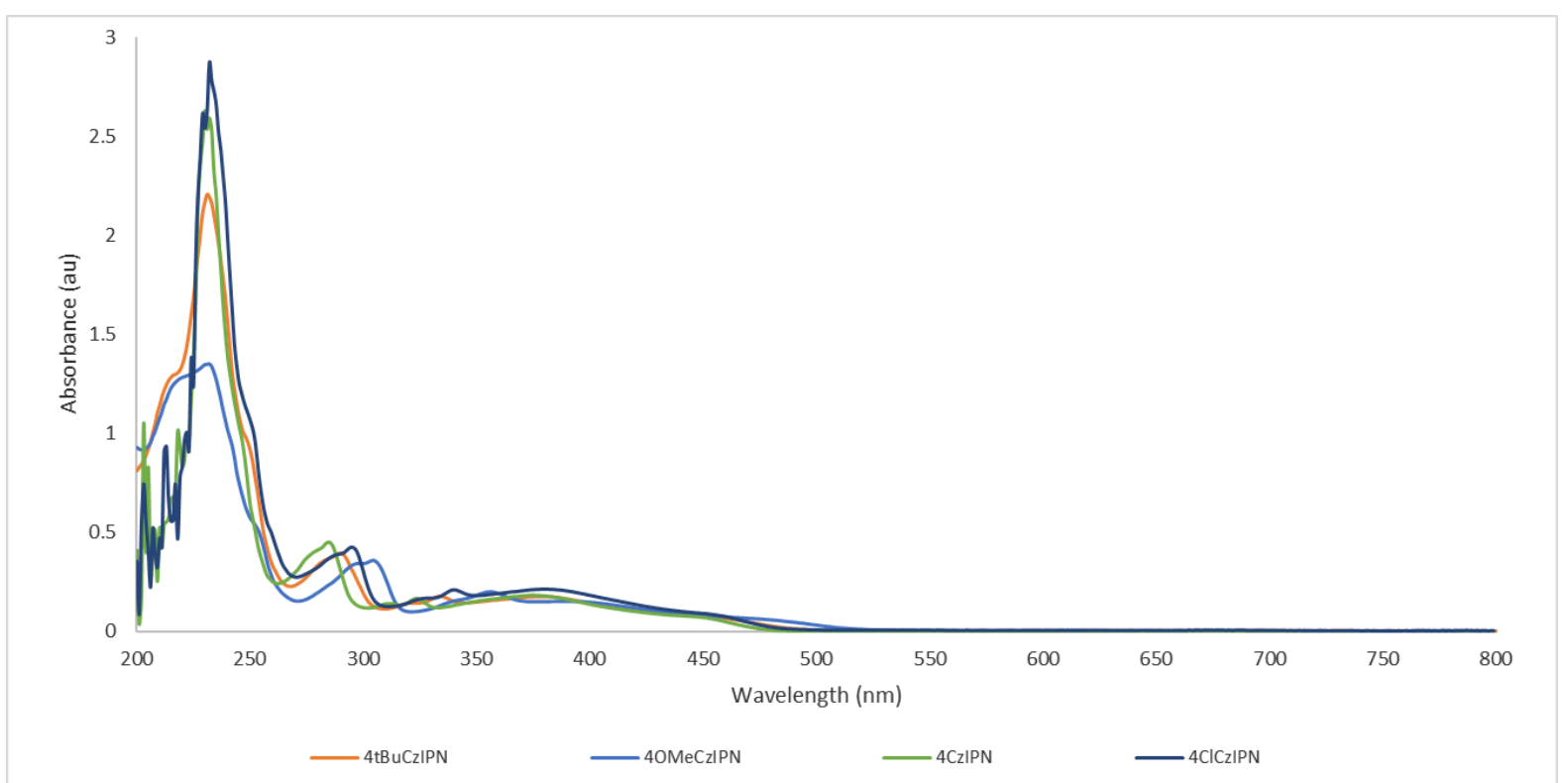

Figure S1: UV-Vis spectra of 4CzIPN derivatives 2 in acetonitrile

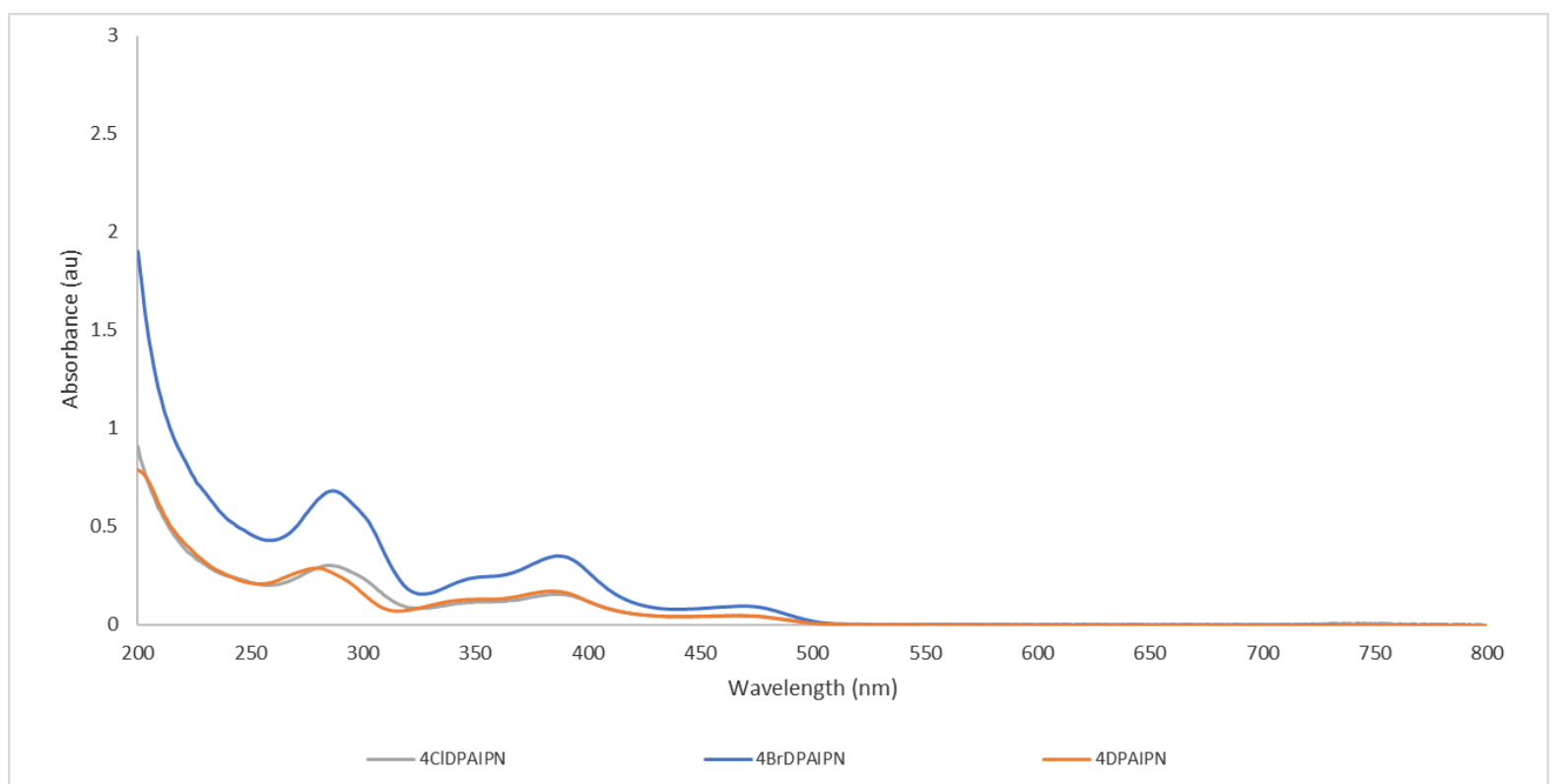

Figure S2: UV-Vis spectra of 4DPAIPN derivatives 3 in acetonitrile 


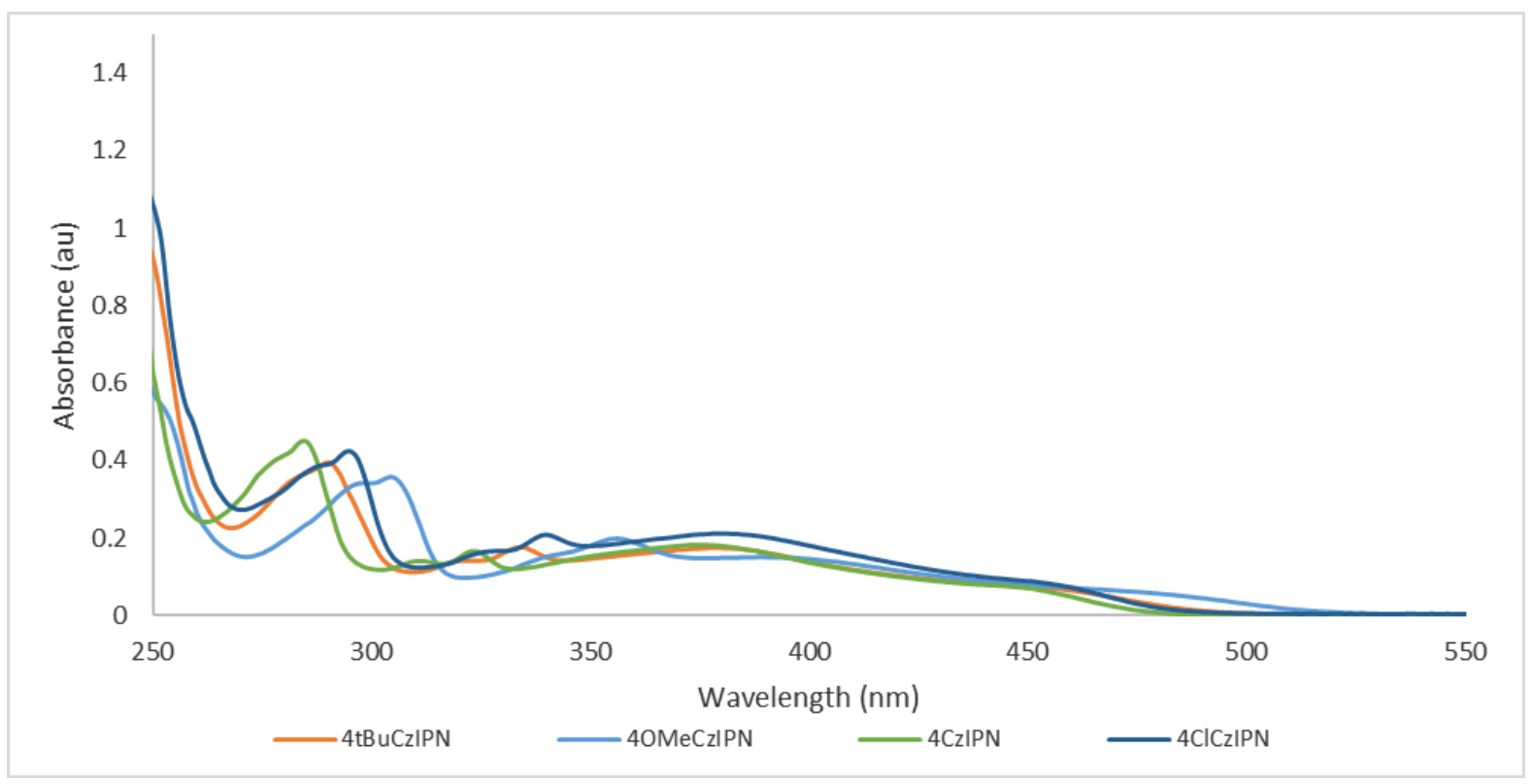

Figure S3: Zoom on the 250-550 nm of the UV-Vis spectra of 4CzIPN derivatives 2 in acetonitrile

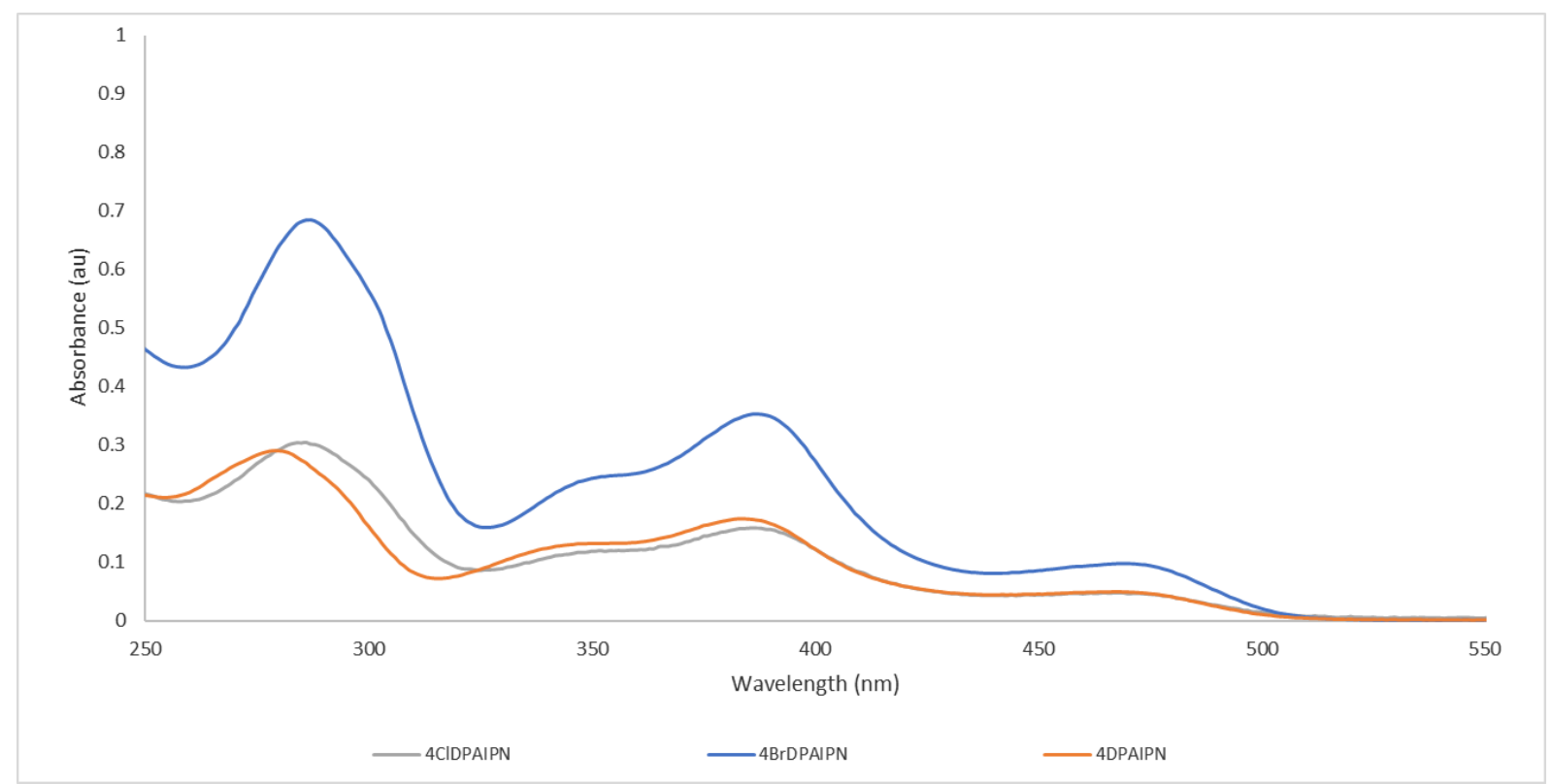

Figure S4: Zoom on the $250-550 \mathrm{~nm}$ of the UV-Vis spectra of 4DPAIPN derivatives 3 in acetonitrile 


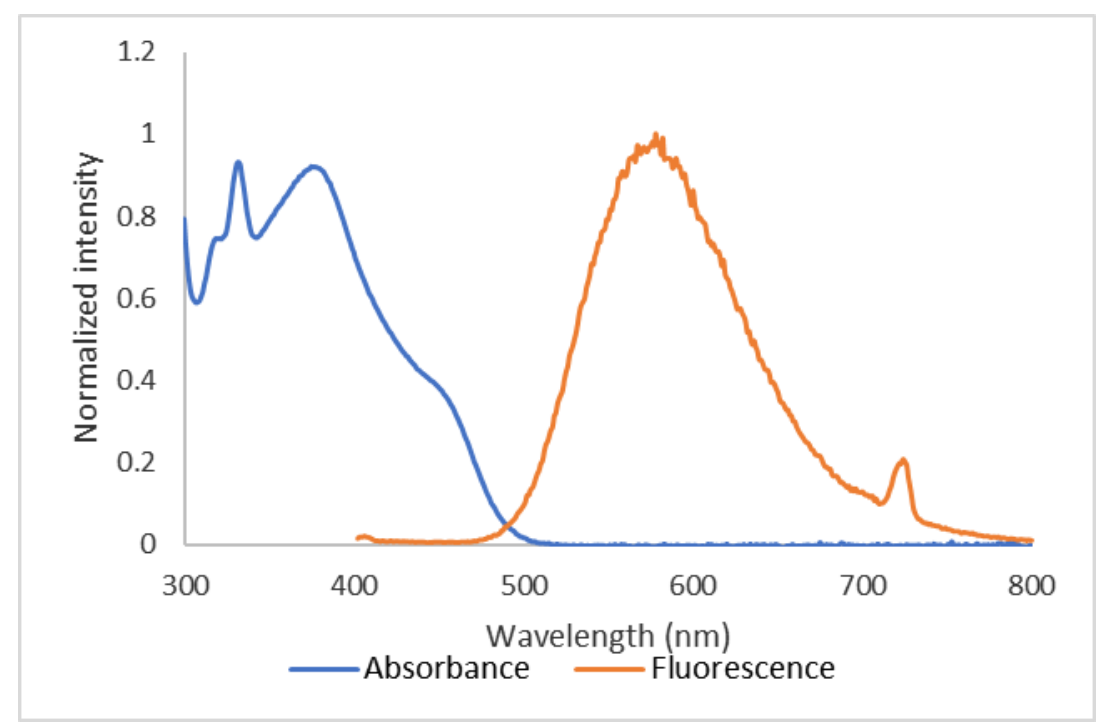

Figure S5 : Absorbance and emission of 4tBuCzIPN (2c) at 10 umol.L-1 in acetonitrile

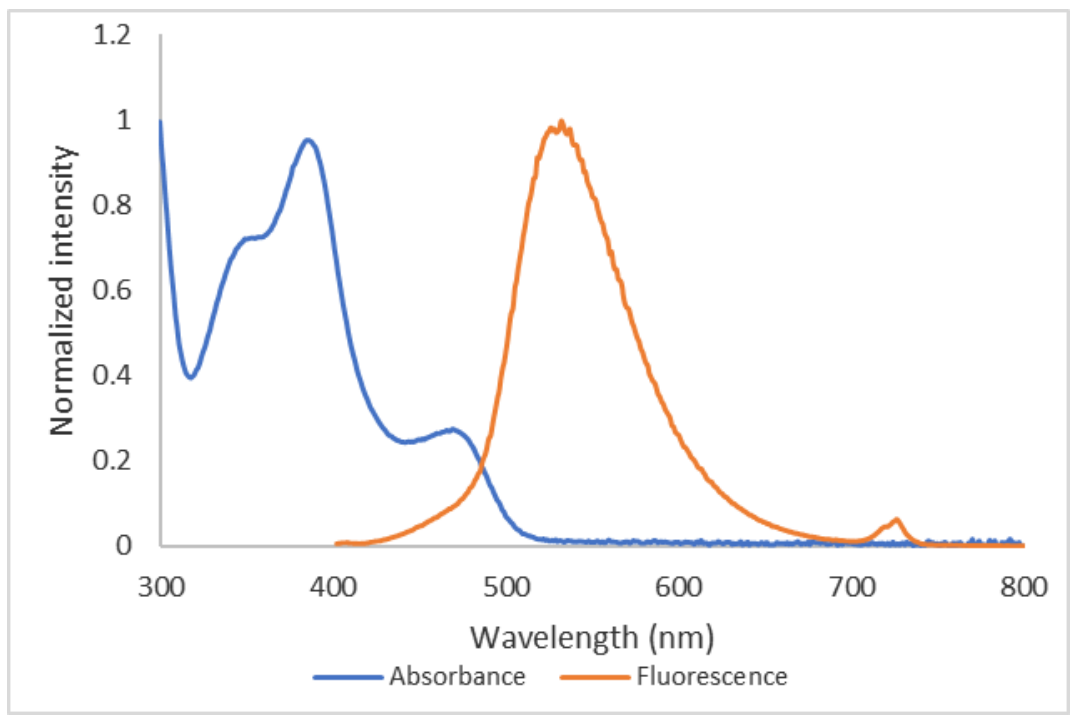

Figure S6 : Absorbance and emission of 4DPAIPN (3a) at 10 umol.L-1 in acetonitrile

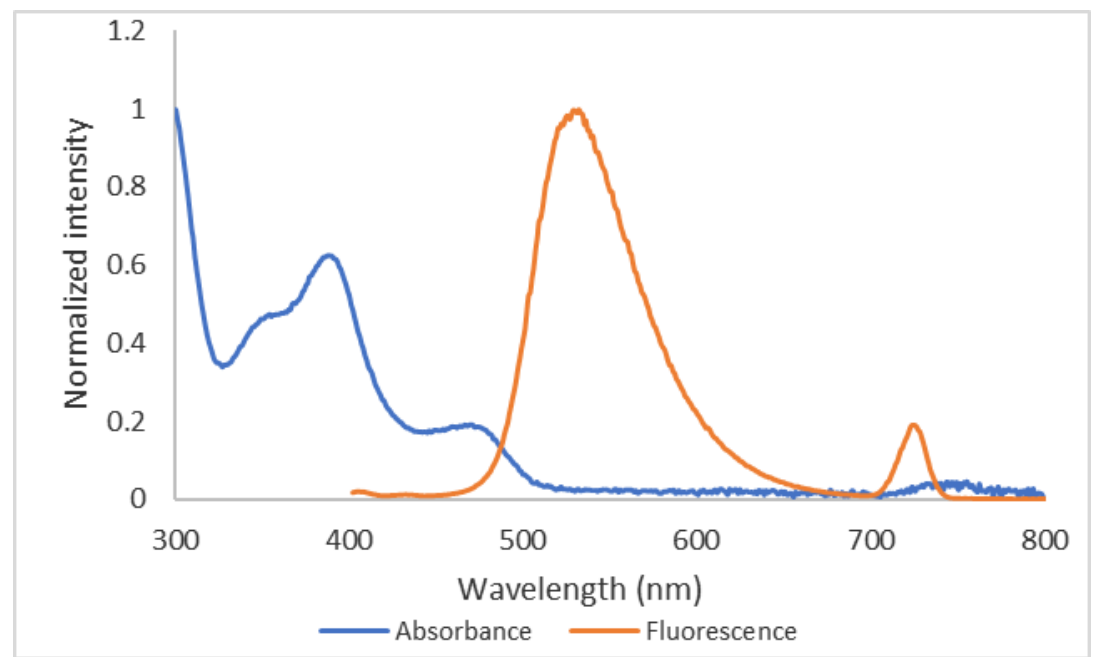

Figure S7 : Absorbance and emission of 4CIDPAIPN (3b) at 10 umol.L-1 in acetonitrile 


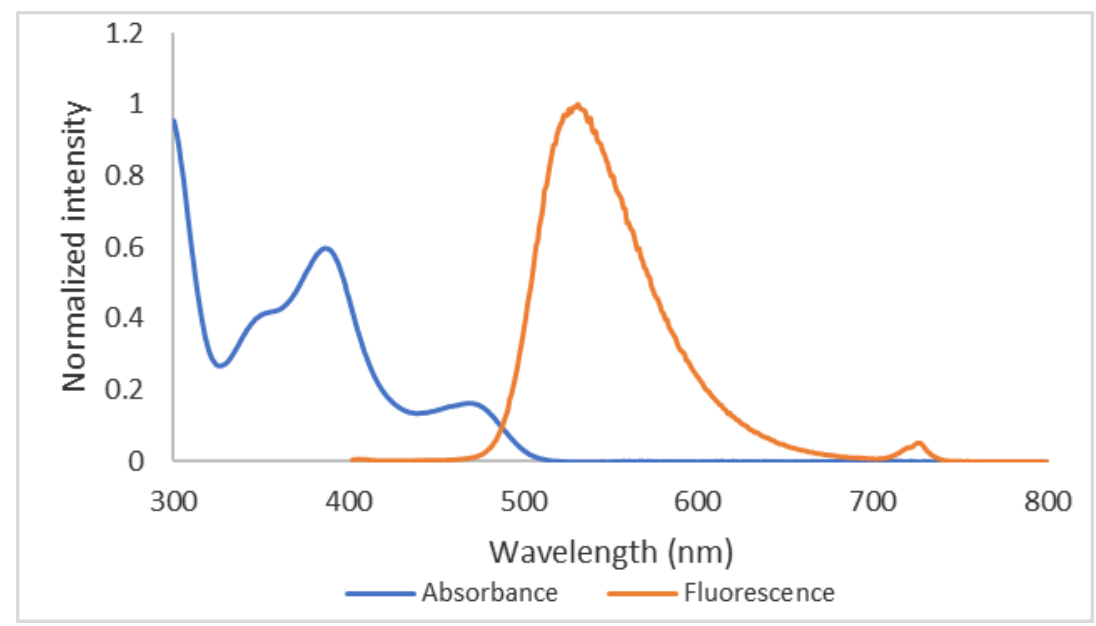

Figure S8: Absorbance and emission of 4BrDPAIPN (3c) at 10 umol.L-1 in acetonitrile

\subsection{Electrochemical measurements}

The cyclic voltammetry of the organic dyes were performed. For comparison, the organic dyes were studied in the same conditions as previous reports on 4CzIPN, at $1 \mathrm{mM}$ in degassed acetonitrile at scan rates of $0.1,0.5$ and $1 \mathrm{~V} / \mathrm{s} .{ }^{[10,17,19]}$ For clarity, curves are presented at $0.1 \mathrm{~V} / \mathrm{s}$ as well as a superposition.

In the case of 4CzIPN derivatives, irreversible waves were observed in the anodic scan and correspond to the oxidation of the carbazole moieties, as reported by Ishimatsu et al. ${ }^{[19]}$ The same group described that the introduction of $t$ Bu groups prevents this polymerization. ${ }^{[14]}$ Reversibility was evaluated through plots of the intensity of the peaks related to the square root of the scan rate. ${ }^{[20]}$ Reversible waves were observed in the cathodic scans in all cases. They correspond to the reduction of the dicyanobenzene group. ${ }^{[14]}$ For 4BrDPAIPN (3b), higher scan rates gave surprising curves for the reduction, hence the reversibility could not be determined by this method. With a peak to peak separation of $60 \mathrm{mV}$, this process in quasi-reversible.

In the conditions of this study, the expected anodic and cathodic shifts upon substitution effect were measured (Table S1). The trend in reduction potentials of 4DPAIPN derivatives $(3 \mathbf{a}<\mathbf{3 b}<\mathbf{3 c})$ follows the electronegativity of the substituents $(\mathrm{H}<\mathrm{Br}<\mathrm{Cl})$. This can be rationalized by a greater stabilization of the lowest unoccupied molecular orbital (LUMO) with substitution. ${ }^{[10]}$ Similarly, 4tBuCzIPN (2c) and 4 OMeCzIPN (2d) were less oxidant than 4CzIPN (2a) by destabilization of the LUMO.

The excitation energy $E_{0-0}$ was estimated by the point of intersection of the normalized absorbance and emission signals (Section 4.1). $E_{1 / 2}\left(P^{+} / P^{\star}\right)=E_{1 / 2}\left(P^{+} / P\right)-E_{0-0}$ and $E_{1 / 2}\left(P^{\star} / P^{-}\right)=E_{0-0}+E_{1 / 2}\left(P / P^{-}\right) .[21]$

Table S1: Electrochemical properties of 4CzIPN and DPAIPN derivatives 2 and $\mathbf{3}^{a}$

\begin{tabular}{cccccc}
\hline Photocatalyst & $\mathrm{E}_{0-0}(\mathrm{eV})$ & $\mathrm{E}_{1 / 2}\left(\mathrm{P} / \mathrm{P}^{-}\right)$ & $\mathrm{E}_{1 / 2}\left(\mathrm{P}^{*} / \mathrm{P}^{-}\right)$ & $\mathrm{E}_{1 / 2}\left(\mathrm{P}^{+} / \mathrm{P}\right)$ & $\mathrm{E}_{1 / 2}\left(\mathrm{P}^{+} / \mathrm{P}^{*}\right)$ \\
\hline 4CzIPN (2a) & 2.53 & -1.21 & +1.35 & +1.52 & -1.04 \\
4CzIPN (2a) & 2.64 & -1.32 & +1.32 & +1.39 & -1.25 \\
4tBuCzIPN (2c) & 2.53 & -1.32 & +1.21 & +1.22 & -1.31 \\
4OMeCzIPN (2d) & $2.61^{[16]}$ & -1.38 & +1.23 & +1.05 & -1.56 \\
4OMeCzIPN (2d) $)^{[16]}$ & 2.61 & -1.50 & +1.27 & +1.11 & -1.34 \\
4CIDPAIPN (3b) & 2.53 & -1.44 & +1.09 & +1.23 & -1.30 \\
4BrDPAIPN (3c) & 2.53 & -1.55 & +0.98 & +1.12 & -1.41 \\
"4DPAIPN" (3a) ${ }^{[17]}$ & 2.62 & -1.52 & +1.10 & +1.34 & -1.28 \\
4DPAIPN (3a) & 2.55 & -1.65 & +0.90 & +1.03 & -1.52 \\
\hline
\end{tabular}

apotentials in V vs SCE, wavelength in nanometers. 


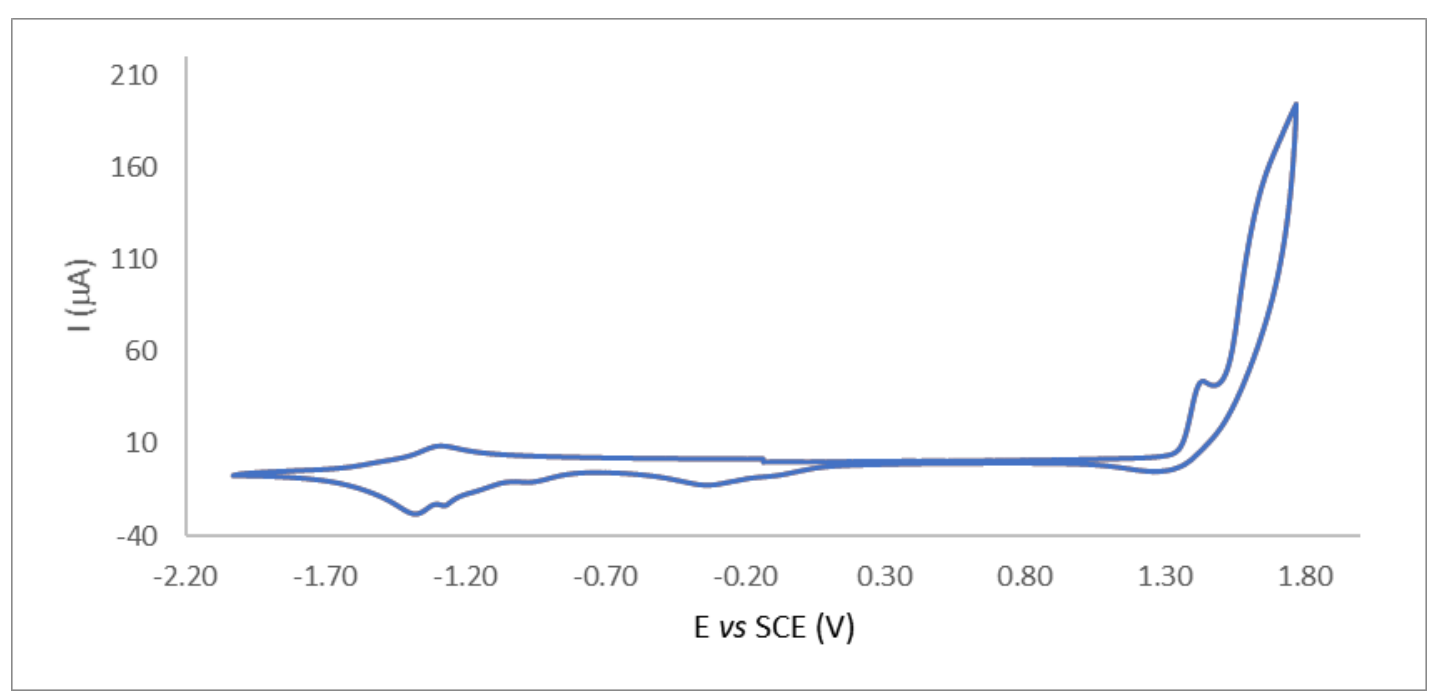

Figure S9: Cyclic voltammetry of $4 C z I P N(2 a)$ in acetonitrile at $0.1 \mathrm{~V} / \mathrm{S}$

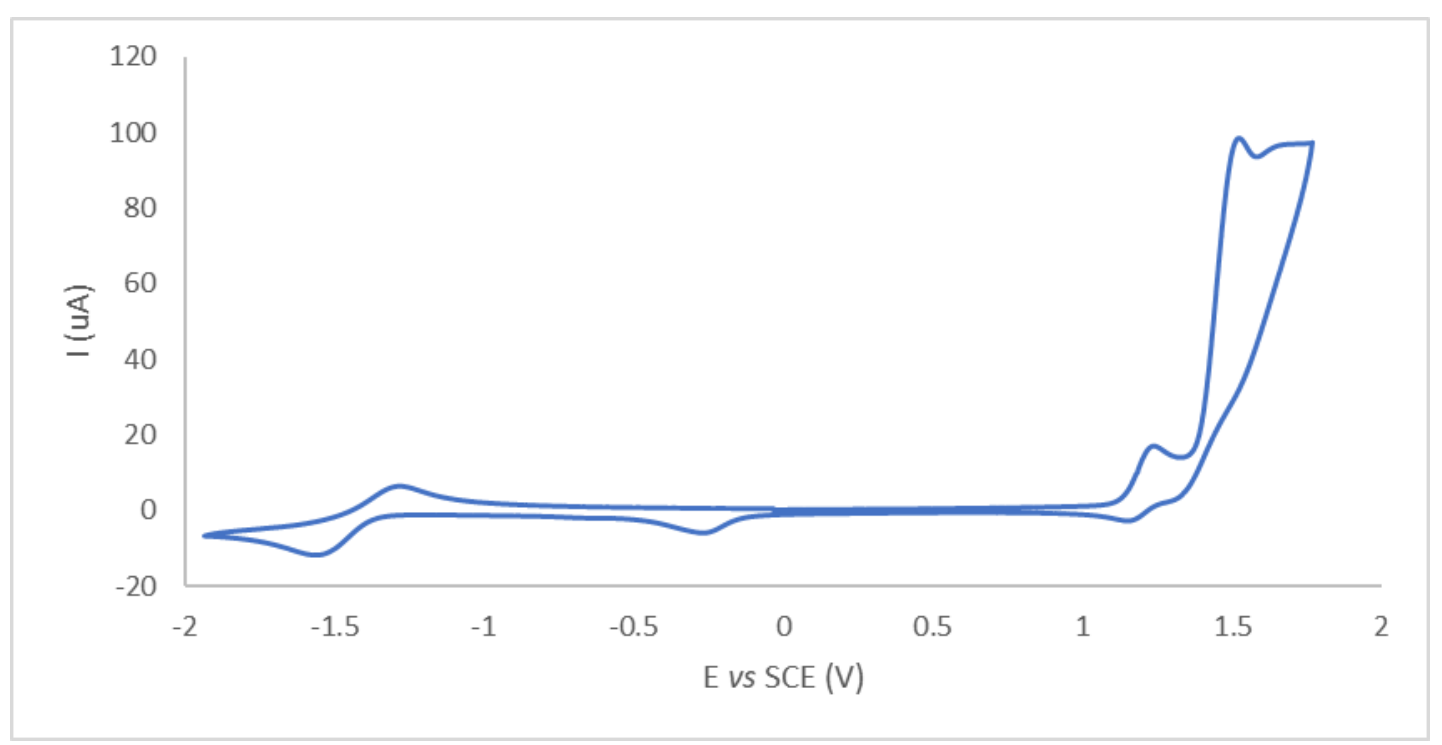

Figure S10 : Cyclic voltammetry of 4tBuCzIPN (2c) in acetonitrile $0.1 \mathrm{~V} / \mathrm{S}$

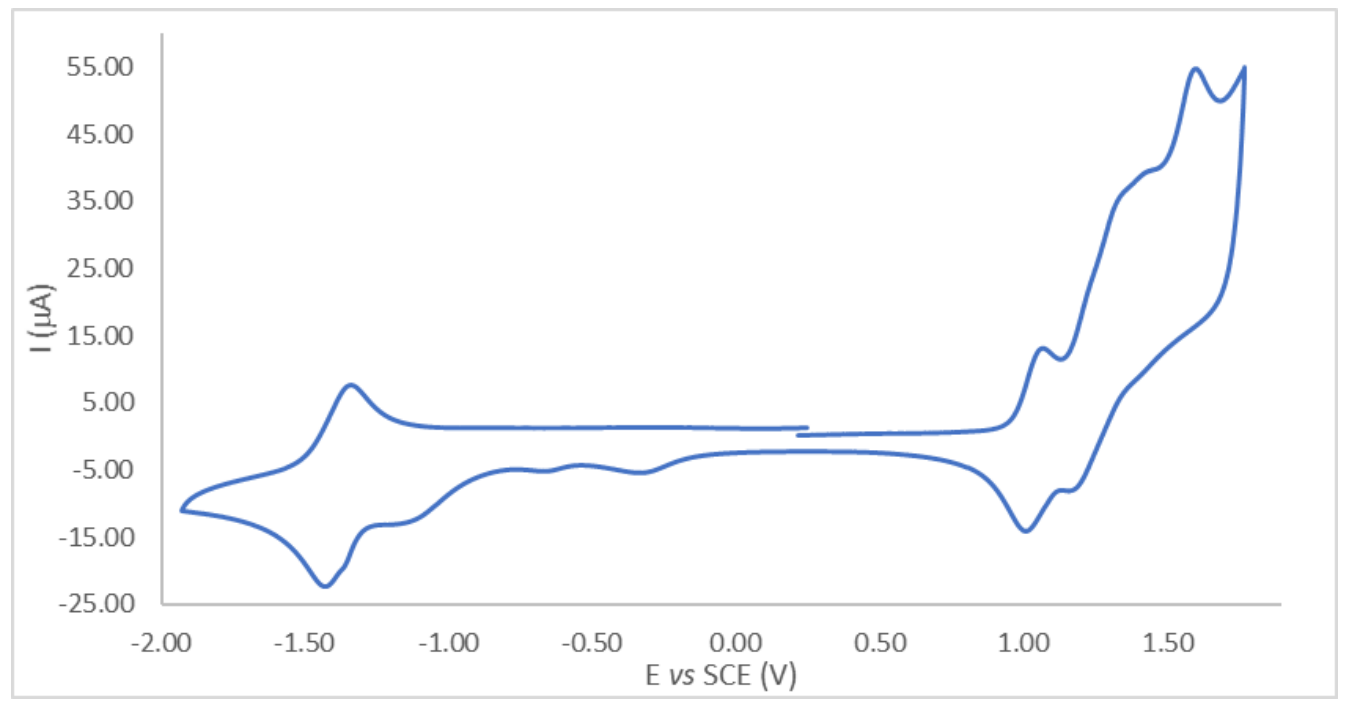

Figure S11: Cyclic voltammetry of $4 O M e C Z I P N(2 d)$ in acetonitrile $0.1 \mathrm{~V} / \mathrm{S}$ 


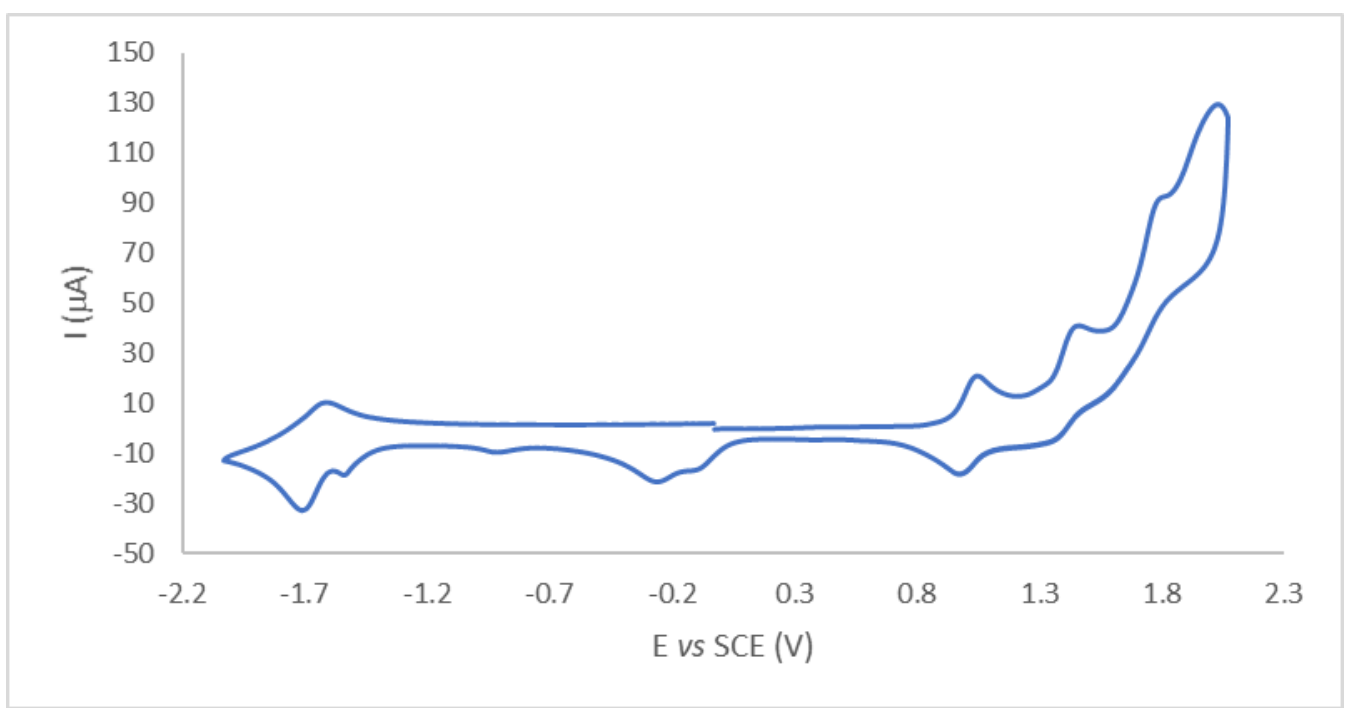

Figure S12: Cyclic voltammetry of $4 D P A I P N(3 a)$ in acetonitrile $0.1 \mathrm{~V} / \mathrm{S}$

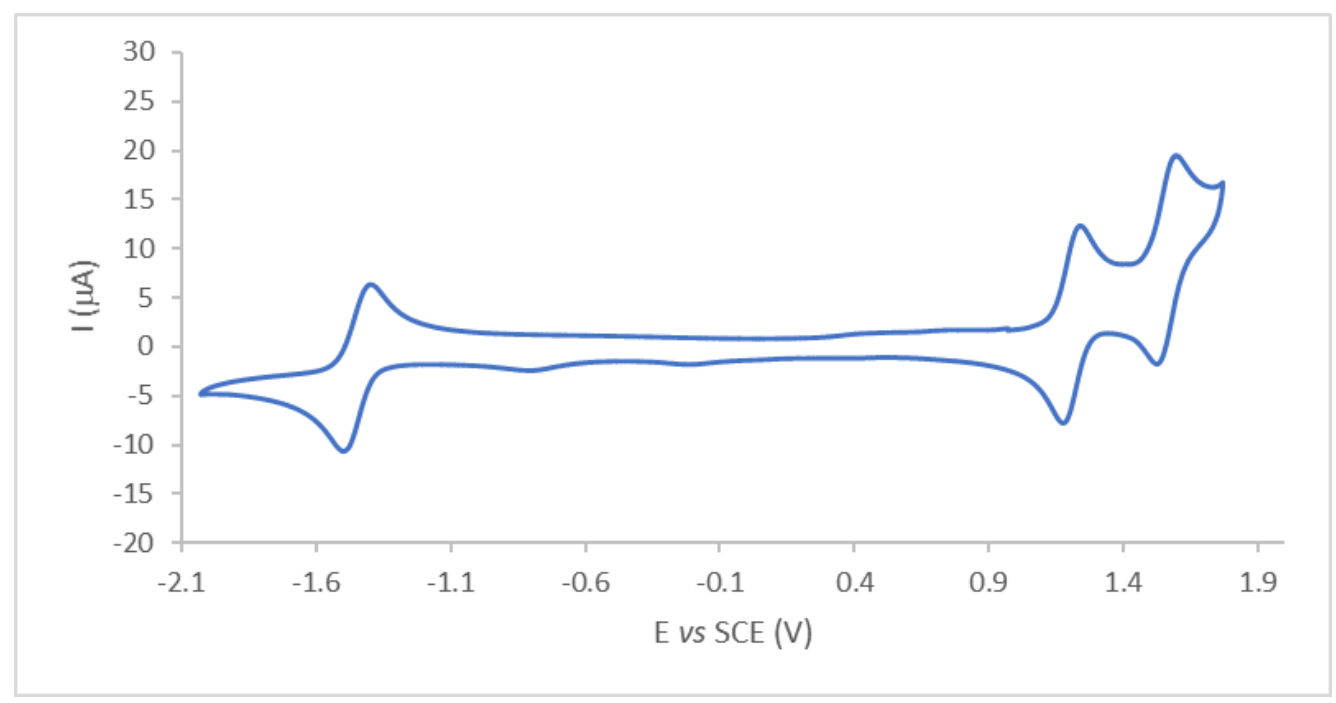

Figure S13: Cyclic voltammetry of 4 CIDPAIPN (3b) in acetonitrile $0.1 \mathrm{~V} / \mathrm{S}$

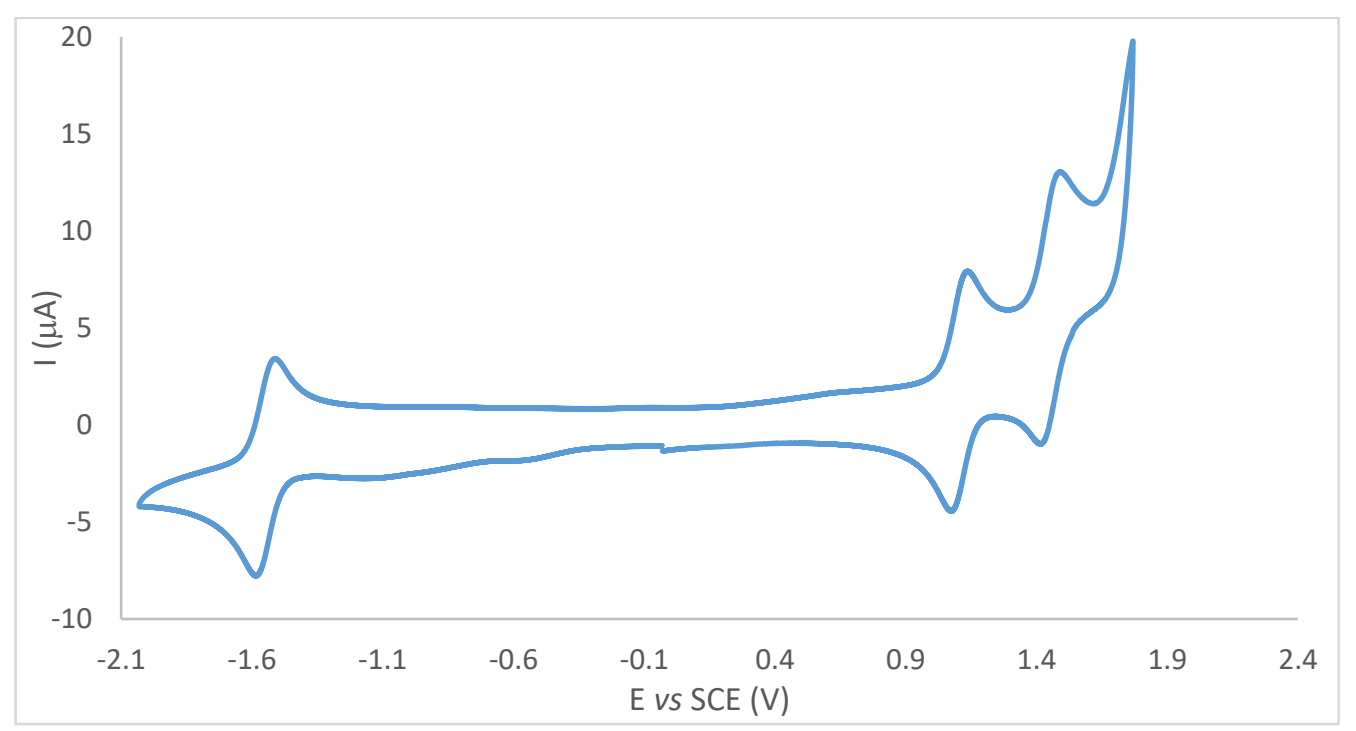

Figure S14: Cyclic voltammetry of 4BrDPAIPN (3c) in acetonitrile $0.1 \mathrm{~V} / \mathrm{S}$ 
Graphs for reversibility determination.

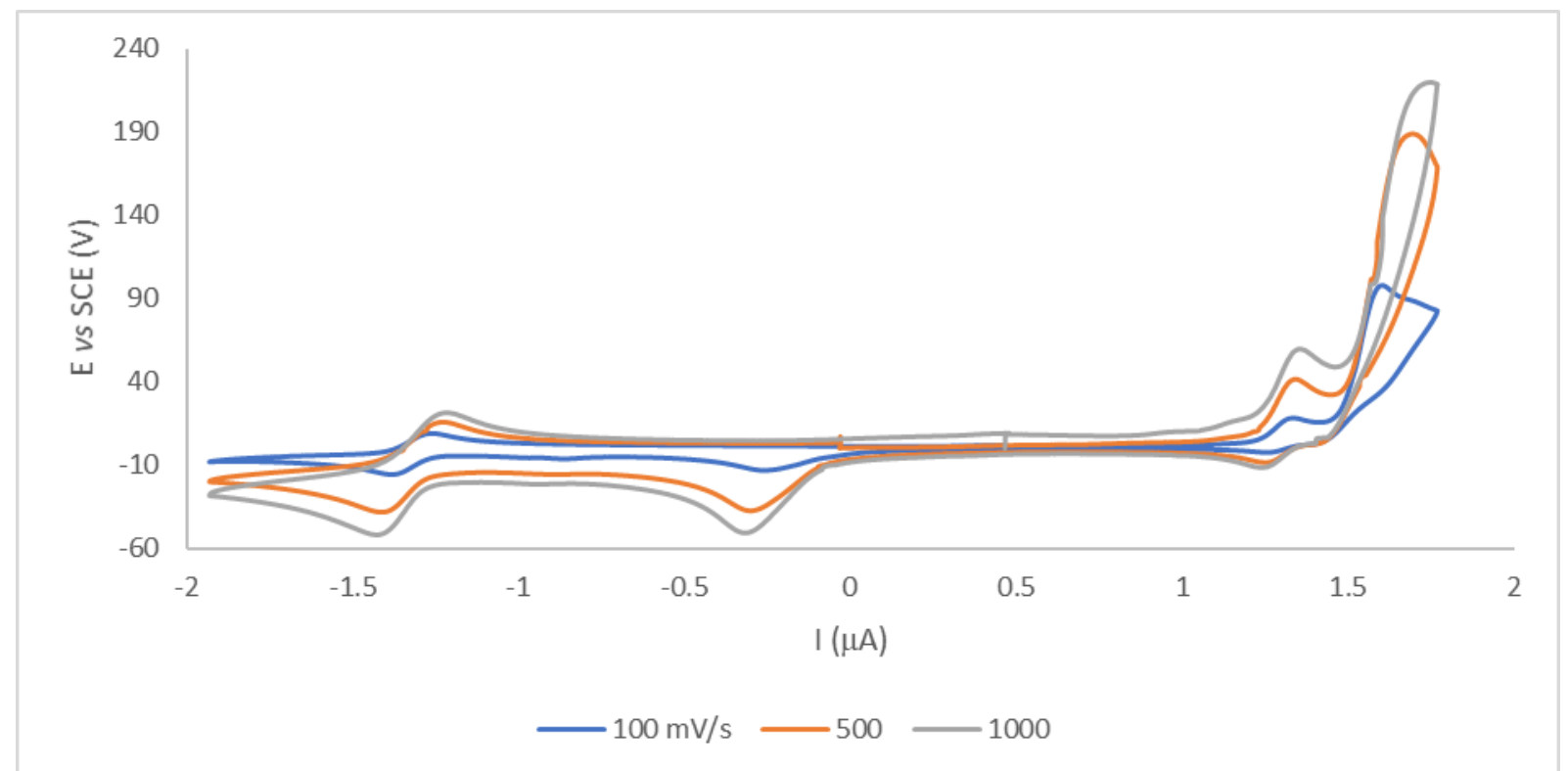

Figure 15: Cyclic voltammetry of 4tBuCzIPN (2c)

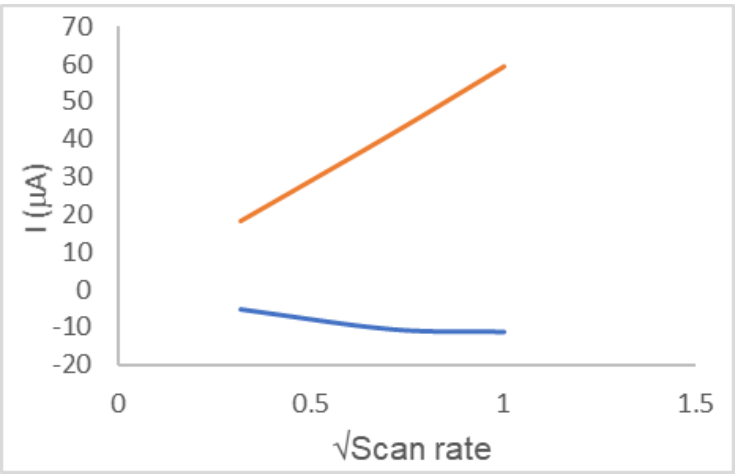

Figure 16: Intensity vs $\sqrt{ }$ scan rate, 4tBuCzIPN-Red

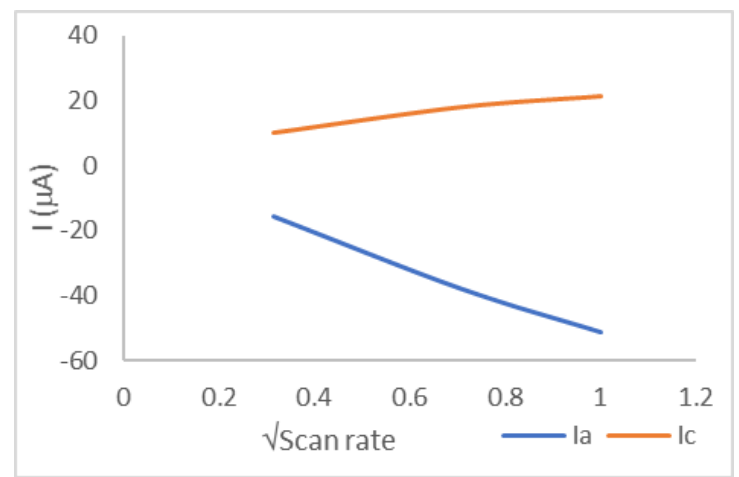

Figure 17: Intensity vs $\sqrt{s c a n}$ rate, $4 t B u C z I P N-O x$ 


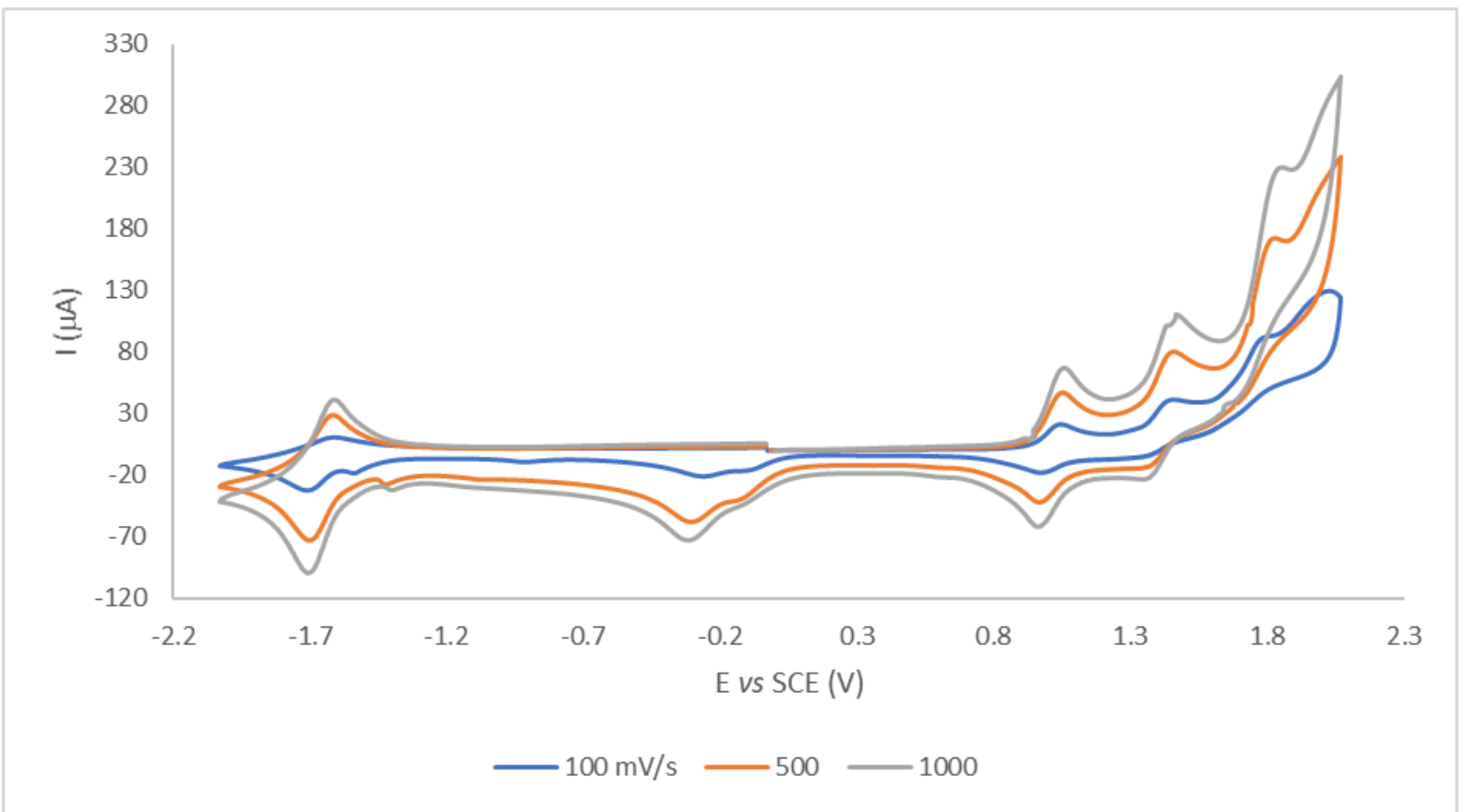

Figure 18: Cyclic voltammetry of 4DPAIPN (3a)

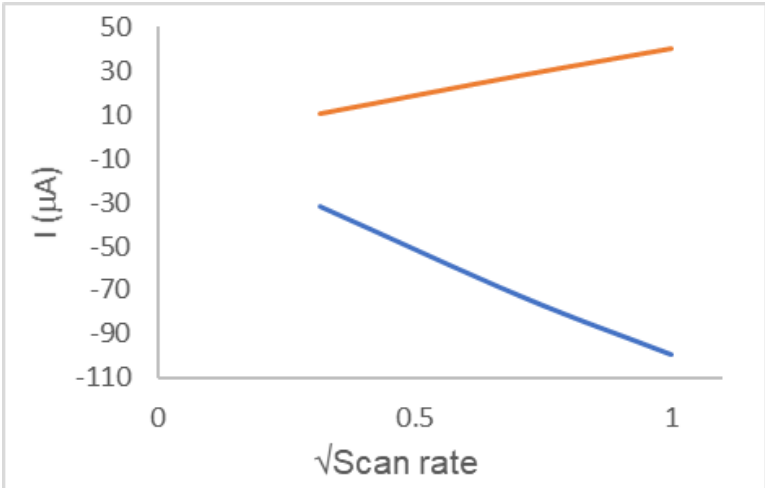

Figure 19: Intensity vs $\sqrt{ }$ scan rate, 4DPAIPN-Red

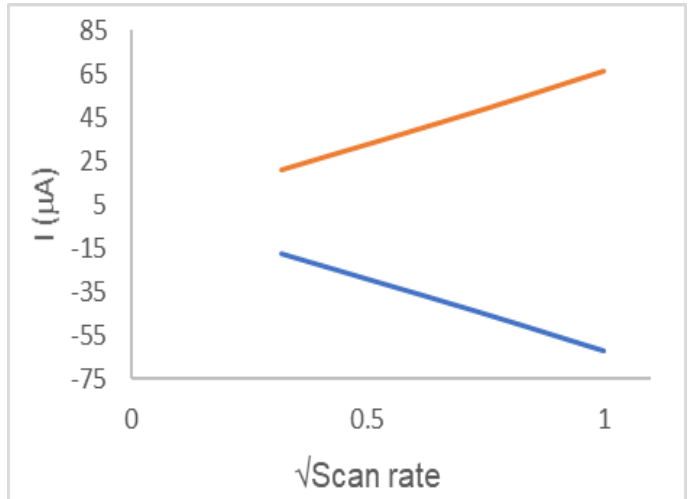

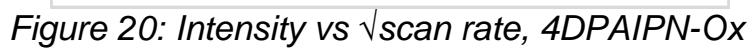




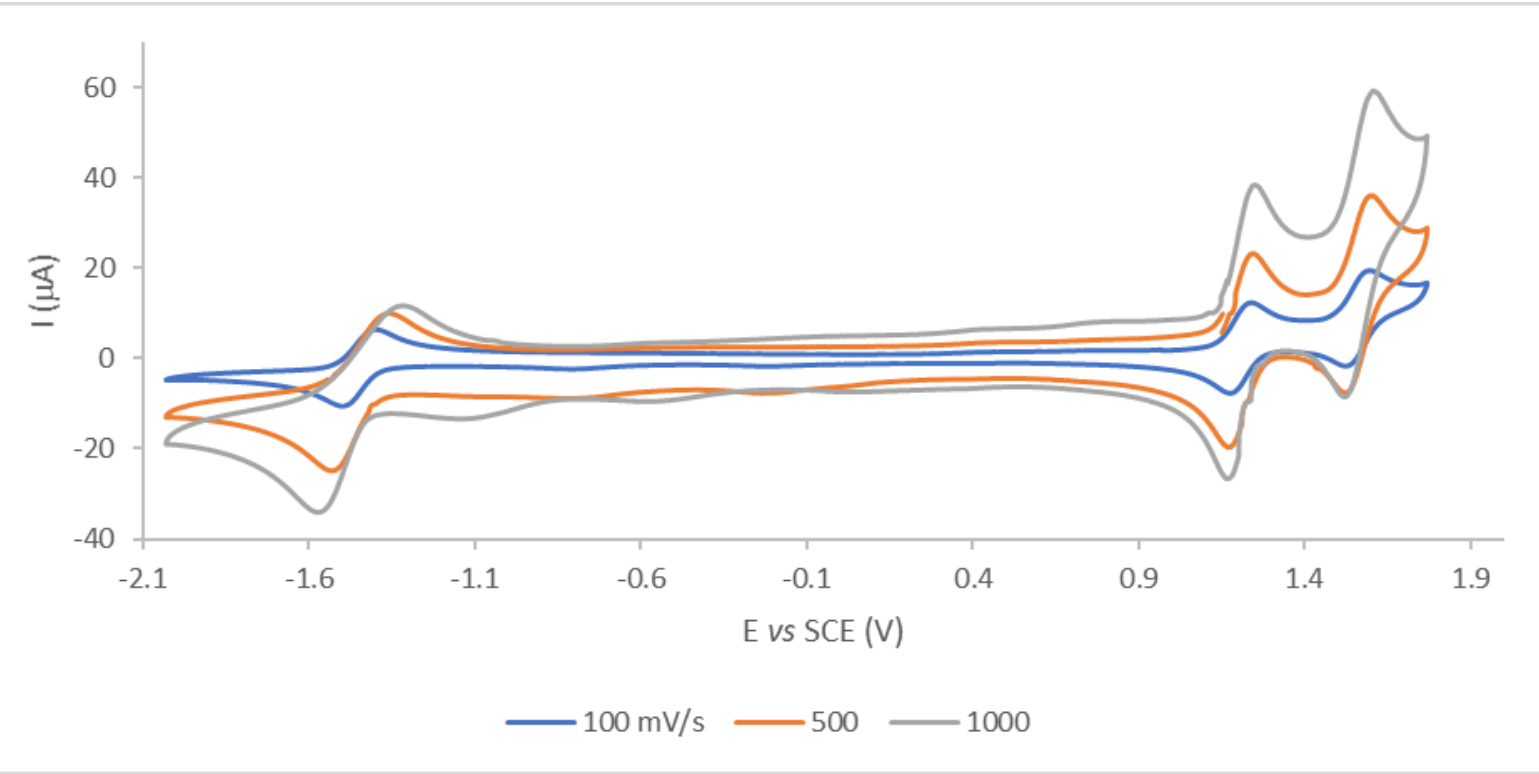

Figure 21: Cyclic voltammetry of 4 CIDPAIPN (3b)

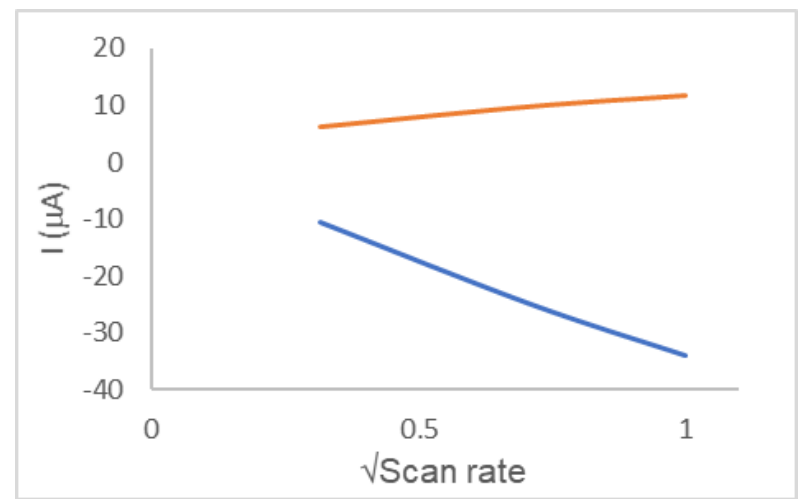

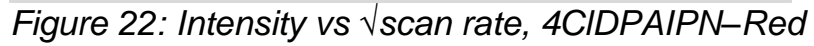

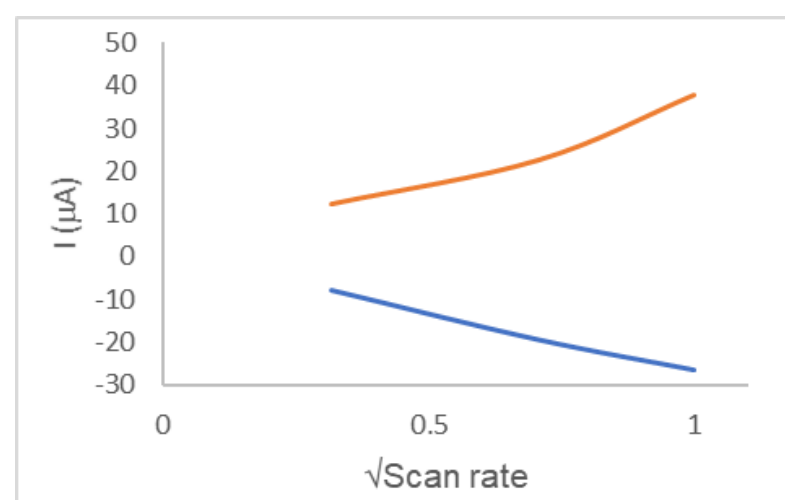

Figure 23: Intensity vs $\sqrt{ }$ scan rate, 4CIDPAIPN-Ox 


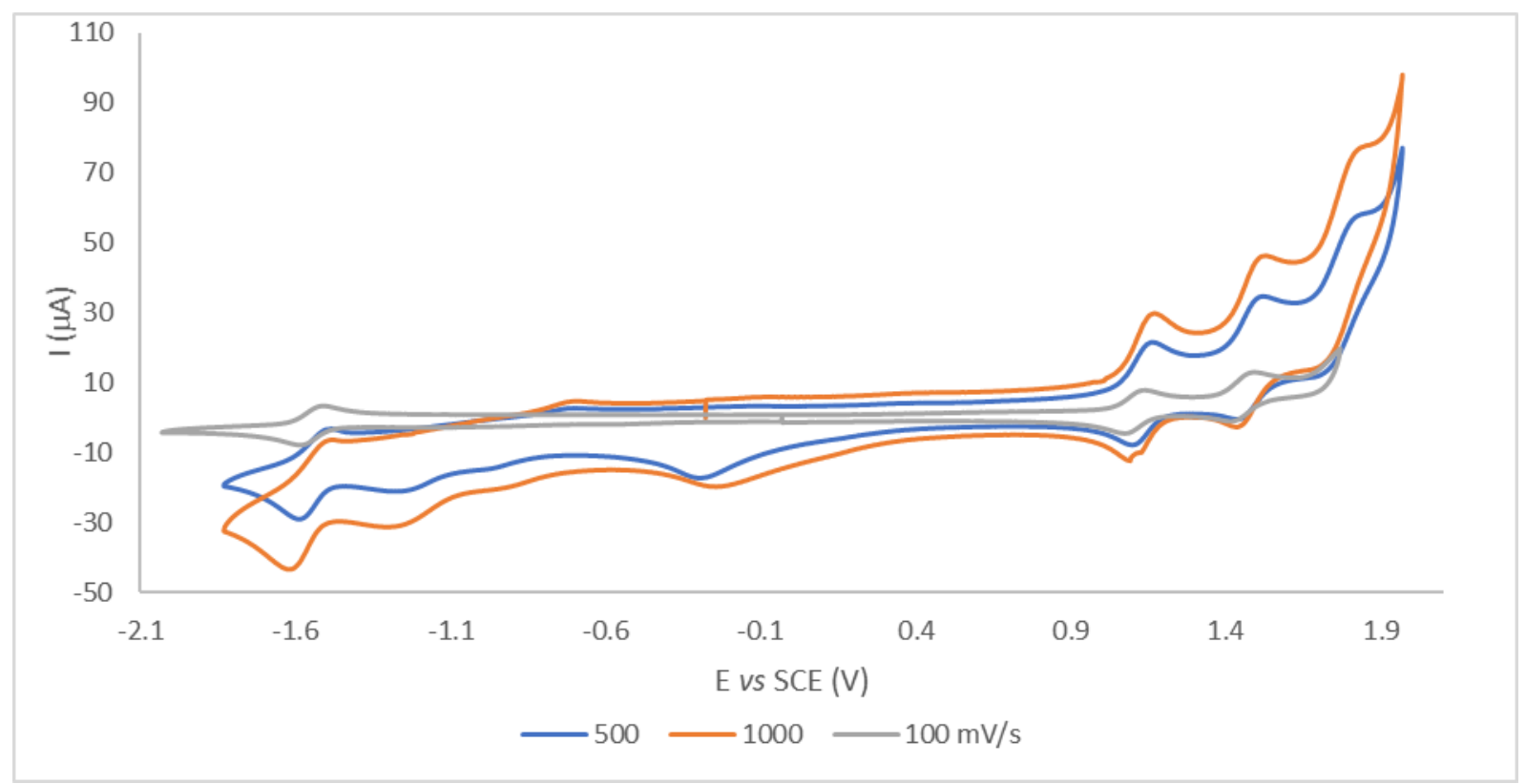

Figure 24: Cyclic voltammetry of 4BrDPAIPN (3c)

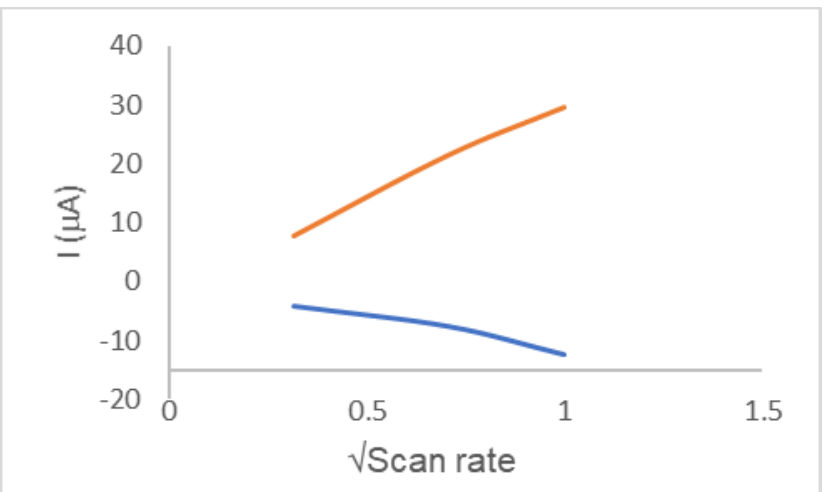

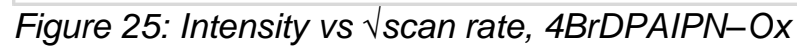




\section{Synthesis of peptide tetramers and Ac-GRGDNP}

All peptide tetramers were synthesized by solid phase peptide synthesis using a 2-chlorotrityl chloride resin (1.0-1.6 mmol/g, 100-200 mesh). The first amino acid was loaded on the resin by incubation of the Fmoc-protected monomer (3 equiv of the number of active sites on the resin), DIPEA (4 equiv) in dichloromethane for $2 \mathrm{~h}$. A cycle consisted first of the deprotection, achieved by stirring for 20 min with a $20 \%$ solution of piperidine in DMF, twice. Then the resin was washed with DMF (7x). Double couplings were performed by adding the Fmoc-protected monomer (4 equiv), HBTU (4 equiv), HOBt (4 equiv), NMM (4 equiv) and stirring for 45 min. Capping was carried out at the end of each cycle, followed by a DMF wash (7x). Acetylation of the N-terminal was achieved by incubating the resin with an Acetic Anhydride/DIPEA/DMF 10/15/75 solution for 30 min, twice. Cleavage of peptides with no protecting groups on the side-chains was performed by stirring the resin in a $20 \%$ solution of HFIP in dichloromethane for $30 \mathrm{~min}$. In the presence of protecting groups, a TFA/water/triisopropylsilane 95/2.5/2.5 was used instead and the stirring time increased to $2 \mathrm{~h}$. The cleavage mixture was poured into cold diethyl ether and precipitated peptides were recovered. The crude peptides were purified by preparative RP-HPLC using a gradient water-95\% acetonitrile in 20 min. Pure peptides were analyzed by RP-HPLC and HRMS. 


\section{Decarboxylative alkynylation}

\subsection{Reaction optimization}
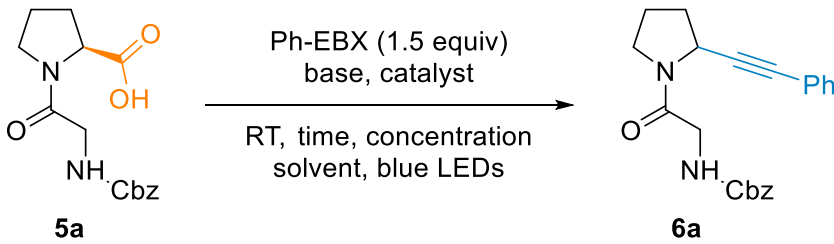

Degassed solvent $(10 \mathrm{~mL})$ was added in a $10 \mathrm{~mL}$ test tube containing a teflon coated stirring bar, ZGly-Pro (5a) (31 mg, $0.10 \mathrm{mmol}, 1.0$ equiv), Ph-EBX (1b) (52 mg, $0.15 \mathrm{mmol}, 1.5$ equiv), the base and the catalyst under a nitrogen atmosphere. The reaction mixture was irradiated using blue light LEDs at RT.

\section{Procedure for HPLC yields:}

$0.5 \mathrm{~mL}$ of the reaction mixture was diluted with acetonitrile ( $3 x$ volume). In the case of metal based catalysts, a filtration over reverse phase silica was performed. The yield was determined using a calibration curve based on absorbance at $214 \mathrm{~nm}$ after a linear regression with Excel. The calibration curve was obtained with samples between 0.25 to $1.25 \mathrm{mg} / \mathrm{mL}$ using the method A described in the general methods.

Procedure for isolated yields:

The crude mixture was diluted with $10 \mathrm{~mL}$ of brine and extracted with diethyl ether $(3 \times 50 \mathrm{~mL})$. The combined organic layers were washed with brine $(3 \times 20 \mathrm{~mL})$, dried over $\mathrm{MgSO}_{4}$, filtered and concentrated under vacuum. The crude product was purified by preparative TLC (pentane/ethyl acetate $1: 1$ or DCM/ethyl acetate 8:2).

Table S2. Optimization of the decarboxylative alkynylation on dipeptides

\begin{tabular}{|c|c|c|c|c|c|c|c|c|}
\hline Entry & Peptide & Solvent & $\begin{array}{c}\text { Concentration } \\
(\mathrm{mM})\end{array}$ & Catalyst (mol\%) & $\begin{array}{l}\text { Base } \\
\text { (equiv) }\end{array}$ & Time & $\begin{array}{c}\text { Ratio } \\
\text { SM/P[a] }\end{array}$ & $\begin{array}{c}\text { HPLC } \\
\text { yield (\%) }\end{array}$ \\
\hline $1^{[\mathrm{b}]}$ & Z-Gly-Pro & $\mathrm{DCE}^{[\mathrm{c}]}$ & 200 & Ir cat $(4)(1)$ & $\mathrm{CsOBz}(3)$ & $22 \mathrm{~h}$ & - & mixture \\
\hline 2 & Z-Gly-Pro & $\mathrm{THF}[\mathrm{c}]$ & 10 & Ir cat (4) (2) & $\mathrm{Cs}_{2} \mathrm{CO}_{3}(1)$ & $15 \mathrm{~h}$ & $>5: 95$ & $66^{[\mathrm{d}]}$ \\
\hline 3 & Z-Gly-Pro & $\operatorname{MeCN}[\mathrm{c}]$ & 10 & Ir cat (4) (2) & $\mathrm{Cs}_{2} \mathrm{CO}_{3}(1)$ & $15 \mathrm{~h}$ & $>5: 95$ & $55^{[\mathrm{d}]}$ \\
\hline 4 & Z-Gly-Pro & $\mathrm{DMF}^{[\mathrm{c}, \mathrm{e}]}$ & 5 & Ir cat (4) (6) & $\mathrm{K}_{2} \mathrm{HPO}_{4}(2)$ & $15 \mathrm{~h}$ & $>5: 95$ & 86 \\
\hline 5 & Z-Gly-Pro & $\mathrm{DMF}^{[\mathrm{c}, \mathrm{e}]}$ & 5 & 4CzIPN (2a) (6) & $\mathrm{K}_{2} \mathrm{HPO}_{4}(2)$ & $15 \mathrm{~h}$ & $>5: 95$ & 86 \\
\hline 6 & Z-Gly-Pro & $\mathrm{DMF}^{[\mathrm{c}]}$ & 5 & 4CzIPN (2a) (6) & $\mathrm{K}_{2} \mathrm{HPO}_{4}(2)$ & $15 \mathrm{~h}$ & $>5: 95$ & $>95$ \\
\hline 7 & Z-Gly-Pro & $\mathrm{DMF}[\mathrm{c}, \mathrm{e}]$ & 5 & $\operatorname{DCA}(6)$ & $\mathrm{K}_{2} \mathrm{HPO}_{4}(2)$ & $15 \mathrm{~h}$ & $24: 76$ & 33 \\
\hline 8 & Z-Ala-Ala & $\left.\mathrm{DMF}^{\mathrm{c}, \mathrm{e}]}\right]$ & 5 & 4CzIPN (2a) (6) & $\mathrm{K}_{2} \mathrm{HPO}_{4}(2)$ & $2 \mathrm{~h}$ & $>5: 95$ & 83 \\
\hline $9^{[f]}$ & Z-Ala-Ala & $\mathrm{DMF}[\mathrm{c}, \mathrm{e}]$ & 5 & 4CzIPN (2a) (6) & $\mathrm{K}_{2} \mathrm{HPO}_{4}(2)$ & $2 \mathrm{~h}$ & $25: 74$ & 27 \\
\hline 10 & Z-Ala-Ala & $\left.\mathrm{DMF}^{\mathrm{c}, \mathrm{g}]}\right]$ & 10 & 4CzIPN (2a) (3) & $\mathrm{K}_{2} \mathrm{HPO}_{4}(2)$ & $1 \mathrm{~h}$ & $>5: 95$ & $77-90$ \\
\hline 11 & Z-Ala-Ala & $\mathrm{DMF}^{[\mathrm{c}, \mathrm{g}]}$ & 10 & 4CzIPN (2a) (0.5-6) & $\mathrm{K}_{2} \mathrm{HPO}_{4}(2)$ & $30 \mathrm{~min}$ & $>5: 95$ & $65-72$ \\
\hline $12^{[\mathrm{h}]}$ & Z-Ala-Ala & $\mathrm{DMF}[\mathrm{g}]$ & 10 & 4CzIPN (2a) (3) & $\mathrm{K}_{2} \mathrm{HPO}_{4}(2)$ & $30 \mathrm{~min}$ & $>5: 95$ & 86 \\
\hline $13^{[i]}$ & Z-Ala-Ala & $\mathrm{DMF}^{[g]}$ & 10 & 4CzIPN (2a) (3) & $\mathrm{K}_{2} \mathrm{HPO}_{4}(2)$ & $30 \mathrm{~min}$ & $>5: 95$ & 71 \\
\hline
\end{tabular}

[a] Ratio of integration at $214 \mathrm{~nm}$ by RP-HPLC, ${ }^{[b]}$ using TIPS-EBX[[], ${ }^{[c]}$ degassed by nitrogen bubbling, ${ }^{[\mathrm{d}]}$ isolated yield, ${ }^{[\mathrm{e}]}$ DMF/ $\mathrm{H}_{2} \mathrm{O}$ 99:1, [i] $40 \mathrm{~W}$ CFL household bulb, ${ }^{[\mathrm{g}]} \mathrm{DMF} / \mathrm{H}_{2} \mathrm{O}$ 98:2, ${ }^{[\mathrm{h}]}$ freeze-pump-throw degassing, ${ }^{\left[{ }^{[i]}\right.}$ non-degassed solvents. 
With the optimized conditions (Table S2, entry 12), a catalyst screening of the novel organic dyes was performed (Table 3). Degassed (by freeze-pump-throw cycles) DMF (10 mL) and degassed water $(0.20 \mathrm{~mL})$ were added in a $15 \mathrm{~mL}$ microwave tube containing a teflon coated stirring bar, CbzGly-Pro (5a) (15.3 mg, $0.0500 \mathrm{mmol}, 1.0$ equiv), Ph-EBX (1b) (26.1 mg, $0.0750 \mathrm{mmol}, 1.5$ equiv), $\mathrm{K}_{2} \mathrm{HPO}_{4}(17.4 \mathrm{mg}, 0.100 \mathrm{mmol}, 2.0$ equiv) and the organic dye (3 mol\%) under a nitrogen atmosphere. The reaction mixture was irradiated using blue light LEDs at RT for $30 \mathrm{~min}$.
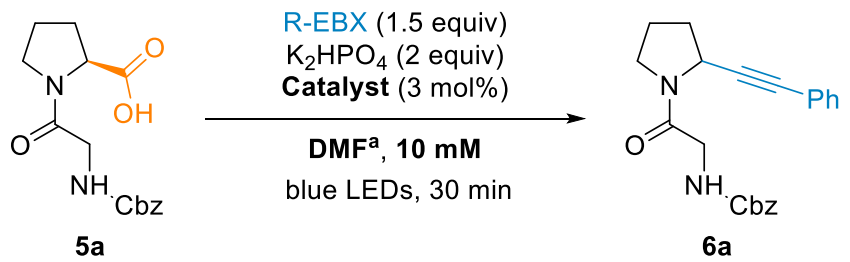

Table 3: Catalyst screening on Z-Gly-Pro

\begin{tabular}{|c|c|c|}
\hline Entry & Catalyst & HPLC yield (\%) \\
\hline 1 & 4CzIPN (2a) & 99 \\
\hline 2 & 4CICzIPN (2b) & 89 \\
\hline 3 & 4DPAIPN (3a) & 43 \\
\hline 4 & 4BrDPAIPN (3c) & 50 \\
\hline 5 & 4CIDPAIPN (3b) & 65 \\
\hline 6 & 4tBuCzIPN (2c) & 44 \\
\hline 7 & $4 \mathrm{MeOCzIPN}(\mathbf{2 d})$ & $<5$ \\
\hline 8 & $4 t \mathrm{BuCzIPN}(2 \mathrm{c})^{[\mathrm{b}]}$ & 57 \\
\hline 9 & Ir cat $(\mathbf{4})$ & 99 \\
\hline $10^{[c]}$ & 4CzIPN (2a) & 87 \\
\hline
\end{tabular}

Control experiments were carried out and only traces of the desired product were observed in the absence of light or catalyst. Interestingly, the presence of a base is not required for the transformation as $24 \%$ yield was still obtained without addition of base. 


\subsection{Robustness experiments}

Degassed DMF $(10 \mathrm{~mL})$ and degassed water $(0.20 \mathrm{~mL})$ were added in a $15 \mathrm{~mL}$ microwave tube containing a teflon coated stirring bar, Cbz-Gly-Pro (5a) $(31 \mathrm{mg}, 0.10 \mathrm{mmol}, 1.0$ equiv), the protected amino acid (0.10 mmol, 1.0 equiv), $\mathrm{Ph}-\operatorname{EBX}(\mathbf{1 b})\left(52 \mathrm{mg}, 0.15 \mathrm{mmol}, 1.5\right.$ equiv), $\mathrm{K}_{2} \mathrm{HPO}_{4}$ (35 mg, $0.20 \mathrm{mmol}, 2.0$ equiv) and 4CzIPN (2a) $(2.4 \mathrm{mg}, 3.0 \mu \mathrm{mol}, 3 \mathrm{~mol} \%)$ under a nitrogen atmosphere. The reaction mixture was irradiated using blue light LEDs at RT for $30 \mathrm{~min}$.

$0.5 \mathrm{~mL}$ of the crude was filtered under an inverse phase silica pad and eluted with $1.5 \mathrm{~mL}$ of acetonitrile. $1 \mathrm{~mL}$ was injected into HPLC. The yield was determined using a calibration curve based on absorbance at $214 \mathrm{~nm}$ after a linear regression with Excel.

Table 4. Robustness experiments
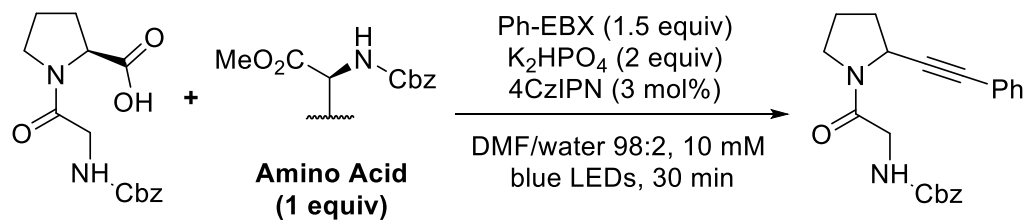

\begin{tabular}{cccc}
\hline Entry & Amino acid (1 equiv) & Ratio SM/Pa & HPLC yield (\%) \\
\hline 1 & Cbz-Met-OMe & $>5: 95$ & 117 \\
2 & Cbz-Ser-OMe & $5: 95$ & 108 \\
3 & Cbz-His-OMe & $6: 94$ & 81 \\
4 & Cbz-Arg-OMe & $19: 81$ & 79 \\
5 & Cbz-Tyr-OMe & $43: 57$ & 43 \\
6 & Cbz-Val-Trp-OMe & $65: 35$ & 35 \\
7 & Cbz-Gln-OMe & $55: 44$ & 60 \\
8 & Cbz-Lys-OMe & $42: 58$ & 58 \\
9 & (Cbz-Cys-OMe)2 & $43: 57$ & 50 \\
& & $2 \mathrm{~h}: 29: 71$ & $2 \mathrm{~h}: 71$ \\
10 & Cbz-Cys-OMe & $25: 75$ & 62 \\
\hline
\end{tabular}

a Ratio of product compared to remaining starting material by HPLC analysis.

In all cases the reactions were clean, with no byproducts. Unreacted starting material was detected when the yield were low. 

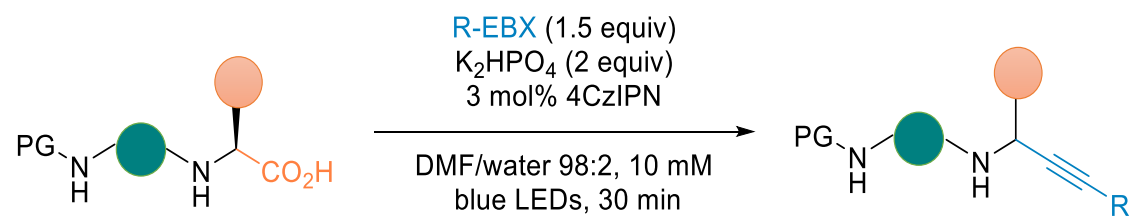

\section{General procedure 3 for the decarboxylative alkynylation of dipeptides}

Degassed DMF and water (30 mL, 98:2) were added in a $50 \mathrm{~mL}$ schlenk flask containing a teflon coated stirring bar, Cbz-Gly-Pro (5a) (92 mg, $0.10 \mathrm{mmol}, 1.0$ equiv), Ph-EBX (1b) $(0.16 \mathrm{~g}, 0.45 \mathrm{mmol}$, 1.5 equiv), $\mathrm{K}_{2} \mathrm{HPO}_{4}(0.11 \mathrm{~g}, 0.60 \mathrm{mmol}, 2.0$ equiv) and $4 \mathrm{CzIPN}$ (2a) $(7.1 \mathrm{mg}, 9.0 \mu \mathrm{mol}, 3 \mathrm{~mol} \%)$ under a nitrogen atmosphere. The reaction mixture was irradiated using blue light LEDs for 30 min at RT.

$0.5 \mathrm{~mL}$ of the crude was filtered under an inverse phase silica pad and eluted with $1.5 \mathrm{~mL}$ of acetonitrile. $1 \mathrm{~mL}$ was injected into HPLC to determine the conversion.

The crude mixture was diluted with $30 \mathrm{~mL}$ of brine and extracted with diethyl ether $(3 \times 150 \mathrm{~mL})$. The combined organic layers were washed with brine $(3 \times 50 \mathrm{~mL})$, dried over $\mathrm{MgSO}_{4}$, filtered and concentrated under vacuum. The crude product was purified by column chromatography DCM to DCM/ethyl acetate 8:2. In some cases a preparative TLC DCM/ethyl acetate 9:1 was peformed for characterization.

\section{Benzyl (2-oxo-2-(2-(phenylethynyl)pyrrolidin-1-yl)ethyl)carbamate (6a)}

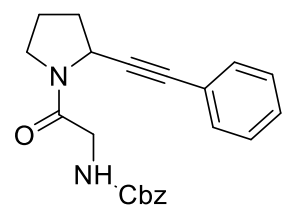

Starting with Cbz-Gly-Pro (5a) (92 $\mathrm{mg}, 0.30 \mathrm{mmol}, 1.0$ equiv), $6 \mathbf{a}$ was obtained after column chromatography DCM to DCM/ethyl acetate 8:2 as a pale yellow oil (100 mg, $0.276 \mathrm{mmol}, 92 \%)$.

$\mathrm{Rf}$ (DCM/ethyl acetate 95:5): 0.3. ${ }^{1} \mathrm{H}$ NMR (400 MHz, Chloroform-d, 6:4 mixture of rotamers (major/minor)) $)^{4} 7.47-7.28(\mathrm{~m}, 10 \mathrm{H}, \mathrm{ArH}$, (major+minor)), $5.84-5.55(\mathrm{~m}, 1 \mathrm{H}, \mathrm{NH}$ (major+minor)), $5.13\left(\mathrm{~s}, 2 \mathrm{H}, \mathrm{OCH} \mathrm{H}_{2} \mathrm{Ph}\right.$ (major+minor)), 5.01 (dd, $J=7.4,2.3 \mathrm{~Hz}, 0.4 \mathrm{H}, \mathrm{NCHC} \equiv \mathrm{C}$ (minor)), $4.69(\mathrm{t}, J=$ $4.9 \mathrm{~Hz}, 0.6 \mathrm{H}, \mathrm{NCHC} \equiv \mathrm{C}$ (major)), 4.31 (dd, $J=17.2,3.8 \mathrm{~Hz}, 0.6 \mathrm{H}, \mathrm{NC}(\mathrm{O}) \mathrm{CH}_{2} \mathrm{NHCbz}$ (major)), 4.18 (dd, $J=17.2,5.1 \mathrm{~Hz}, 0.6 \mathrm{H}, \mathrm{NC}(\mathrm{O}) \mathrm{CH}_{2} \mathrm{NHCbz}$ (major)), $4.06-3.90\left(\mathrm{~m}, 0.8 \mathrm{H}, \quad \mathrm{NC}(\mathrm{O}) \mathrm{CH}_{2} \mathrm{NHCbz}\right.$ (minor)), 3.70 (ddd, $J=11.4,8.0,3.3 \mathrm{~Hz}, 0.6 \mathrm{H}, \mathrm{NCH}_{2}\left(\mathrm{CH}_{2}\right)_{2} \mathrm{CHC} \equiv \mathrm{C}$ (major)), $3.63-3.47(\mathrm{~m}, 1 \mathrm{H}$, $\mathrm{NCH}_{2}\left(\mathrm{CH}_{2}\right)_{2} \mathrm{CHC} \equiv \mathrm{C}$ (major+minor)), $3.44-3.31\left(\mathrm{~m}, 0.4 \mathrm{H}, \mathrm{NCH}{ }_{2}\left(\mathrm{CH}_{2}\right)_{2} \mathrm{CHC} \equiv \mathrm{C}\right.$ (minor)), $2.38-1.91$ (m, $4 \mathrm{H}, \mathrm{NCH}_{2}\left(\mathrm{CH}_{2}\right)_{2} \mathrm{CHC} \equiv \mathrm{C}$ (major+minor)). ${ }^{13} \mathrm{C} \mathrm{NMR}(101 \mathrm{MHz}$, Chloroform-d, mixture of rotamers, signals not fully resolved) $\delta 167.1,166.3,156.2,136.4,131.8,131.7,128.7,128.4,128.3,128.2$, 128.1, 128.0, 128.0, 127.9, 122.7, 121.9, 88.0, 86.8, 84.4, 82.3, 66.8, 48.7, 48.3, 46.1, 45.2, 43.5, 34.5, 32.4, 24.7, 22.9. IR 3293 (w), 2986 (w), 2880 (w), 2362 (w), 2341 (w), 1718 (s), 1648 (s), 1542 $(\mathrm{m}), 1445(\mathrm{~m}), 1263(\mathrm{~m}), 1057(\mathrm{w})$. HRMS (ESI) calcd for $\mathrm{C}_{22} \mathrm{H}_{23} \mathrm{~N}_{2} \mathrm{O}_{3}{ }^{+}[\mathrm{M}+\mathrm{H}]^{+}$363.1703; found 363.1702.

\footnotetext{
${ }^{4}$ The signals of each rotamer were assigned by 2D NMR (see section 7). For clarity, only the spectra of $6 a$ are presented. Following products were assigned by analogy when a 1:1 ratio was measured.
} 


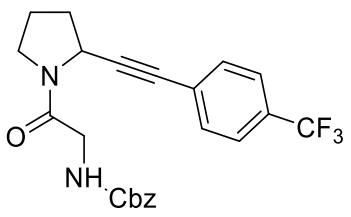

Starting with Cbz-Gly-Pro (5a) (92 mg, $0.30 \mathrm{mmol}, 1.0$ equiv) and $p \mathrm{CF}_{3}$-Ph-EBX (1c) (187 mg, $0.450 \mathrm{mmol}, 1.50$ equiv), $6 \mathrm{~b}$ was obtained after column chromatography DCM to DCM/ethyl acetate $8: 2$ as a yellow oil (124 $\mathrm{mg}$ at $95 \%$ purity, $0.274 \mathrm{mmol}, 91 \%$ ).

$\mathrm{Rf}\left(\mathrm{DCM} /\right.$ ethyl acetate 9:1): 0.3. ${ }^{1} \mathrm{H}$ NMR $\left(400 \mathrm{MHz}\right.$, Chloroform- $d, 1: 1$ mixture of rotamers $\left.\left(\mathrm{R}^{1} / \mathrm{R}^{2}\right)\right)$ ס 7.59 - $7.47\left(\mathrm{~m}, 4 \mathrm{H}, \mathrm{ArH},\left(\mathrm{R}^{1}+\mathrm{R}^{2}\right)\right), 7.45-7.27\left(\mathrm{~m}, 5 \mathrm{H}, \mathrm{ArH},\left(\mathrm{R}^{1}+\mathrm{R}^{2}\right)\right), 5.76$ (bs, $\left.1 \mathrm{H}, \mathrm{NH}\left(\mathrm{R}^{1}+\mathrm{R}^{2}\right)\right)$, $5.12\left(\mathrm{~s}, 2 \mathrm{H}, \mathrm{OCH}_{2} \mathrm{Ph},\left(\mathrm{R}^{1}+\mathrm{R}^{2}\right)\right), 5.01\left(\mathrm{dd}, J=7.3,2.7 \mathrm{~Hz}, 0.5 \mathrm{H}, \mathrm{NCHC} \equiv \mathrm{C}\left(\mathrm{R}^{1}\right)\right), 4.71(\mathrm{t}, J=4.9 \mathrm{~Hz}$, $\left.0.5 \mathrm{H}, \mathrm{NCHC} \equiv \mathrm{C}\left(\mathrm{R}^{2}\right)\right), 4.31\left(\mathrm{dd}, J=17.1,3.9 \mathrm{~Hz}, 0.5 \mathrm{H}, \mathrm{NC}(\mathrm{O}) \mathrm{CH}_{2} \mathrm{NHCbz}\left(\mathrm{R}^{2}\right)\right), 4.15(\mathrm{dd}, J=17.1$, $\left.4.7 \mathrm{~Hz}, 0.5 \mathrm{H}, \mathrm{NC}(\mathrm{O}) \mathrm{CH}_{2} \mathrm{NHCbz}\left(\mathrm{R}^{2}\right)\right), 3.99\left(\mathrm{~d}, J=4.3 \mathrm{~Hz}, 1 \mathrm{H}, \mathrm{NC}(\mathrm{O}) \mathrm{CH}_{2} \mathrm{NHCbz}\left(\mathrm{R}^{1}\right)\right), 3.75-3.66$ $\left(\mathrm{m}, 0.5 \mathrm{H}, \mathrm{NCH} \mathrm{H}_{2}\left(\mathrm{CH}_{2}\right)_{2} \mathrm{CHC} \equiv \mathrm{C}\left(\mathrm{R}^{2}\right)\right), 3.64-3.46\left(\mathrm{~m}, 1 \mathrm{H} \mathrm{NCH}{ }_{2}\left(\mathrm{CH}_{2}\right)_{2} \mathrm{CHC} \equiv \mathrm{C}\left(\mathrm{R}^{1}+\mathrm{R}^{2}\right)\right)$, $3.46-3.33$ $\left(\mathrm{m}, 0.5 \mathrm{H}, \mathrm{NCH} \mathrm{H}_{2}\left(\mathrm{CH}_{2}\right)_{2} \mathrm{CHC} \equiv \mathrm{C}\left(\mathrm{R}^{1}\right)\right), 2.39-1.93\left(\mathrm{~m}, 4 \mathrm{H}, \mathrm{NCH}_{2}\left(\mathrm{CH}_{2}\right)_{2} \mathrm{CHC} \equiv \mathrm{C},\left(\mathrm{R}^{1}+\mathrm{R}^{2}\right)\right) .{ }^{13} \mathrm{C} \mathrm{NMR}$ (101 MHz, Chloroform- $d$, mixture of rotamers, signals not fully resolved) ${ }^{5} \delta 167.1,166.4,162.0$, 156.2, 136.4, $132.0(\mathrm{~d}, J=3.8 \mathrm{~Hz}), 130.4(\mathrm{~d}, J=33.1 \mathrm{~Hz}), 129.9(\mathrm{~d}, J=32.7 \mathrm{~Hz}), 128.5,128.0(\mathrm{~d}, J$ $=8.8 \mathrm{~Hz}$ ), 126.1 (d, $J=79.2 \mathrm{~Hz}), 125.3(\mathrm{q}, J=4.0 \mathrm{~Hz}), 125.0(\mathrm{q}, J=3.5 \mathrm{~Hz}), 122.5(\mathrm{~d}, J=9.4 \mathrm{~Hz})$, 90.6, 89.2, 83.1, 81.0, 66.9, 48.5, 48.2, 46.2, 45.2, 43.5, 34.4, 32.3, 24.8, 22.9. ${ }^{19} \mathrm{~F}$ NMR (376 MHz, Chloroform-d) $\delta$-62.8, -62.9. IR 3290 (w), $2986(w), 2881(w), 2831(w), 2362(m), 2343(w), 2122$ (w), $1717(\mathrm{~m}), 1653(\mathrm{~s}), 1542(\mathrm{~m}), 1457(\mathrm{w}), 1437(\mathrm{w}), 1326(\mathrm{~s}), 1263(\mathrm{w}), 1170(\mathrm{~m}), 1129(\mathrm{~m}), 1068$ $(\mathrm{m}), 845(\mathrm{w})$. HRMS (ESI) calcd for $\mathrm{C}_{23} \mathrm{H}_{21} \mathrm{~F}_{3} \mathrm{~N}_{2} \mathrm{NaO}_{3}{ }^{+}[\mathrm{M}+\mathrm{Na}]^{+}$453.1396; found 453.1395.

\section{Benzyl (2-(2-((4-bromophenyl)ethynyl)pyrrolidin-1-yl)-2-oxoethyl)carbamate (6c)}

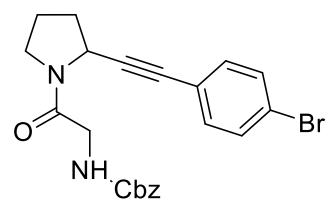

Starting with Cbz-Gly-Pro (5a) (92 mg, 0.30 mmol, 1.0 equiv) and pBr-Ph-EBX (1d) (192 mg, 0.450 mmol, 1.50 equiv), $6 \mathrm{c}$ was obtained after column chromatography DCM to DCM/ethyl acetate 8:2 as a yellow oil (122 $\mathrm{mg}, 0.276 \mathrm{mmol}, 92 \%)$.

$\mathrm{Rf}\left(\mathrm{DCM} /\right.$ ethyl acetate 9:1): 0.3. ${ }^{1} \mathrm{H}$ NMR $\left(400 \mathrm{MHz}\right.$, Chloroform- $d, 1: 1$ mixture of rotamers $\left.\left(\mathrm{R}^{1} / \mathrm{R}^{2}\right)\right)$ ס 7.46 - $7.28\left(\mathrm{~m}, 9 \mathrm{H}, \operatorname{ArH}\left(\mathrm{R}^{1}+\mathrm{R}^{2}\right)\right), 5.78\left(\mathrm{~s}, 1 \mathrm{H}, \mathrm{NH},\left(\mathrm{R}^{1}+\mathrm{R}^{2}\right)\right), 5.12\left(\mathrm{~s}, 2 \mathrm{H}, \mathrm{OCH} \mathrm{H}_{2} \mathrm{Ph},\left(\mathrm{R}^{1}+\mathrm{R}^{2}\right)\right), 4.98$ (dd, $\left.J=7.2,2.5 \mathrm{~Hz}, 0.5 \mathrm{H}, \mathrm{NCHC} \equiv \mathrm{C}\left(\mathrm{R}^{1}\right)\right), 4.67\left(\mathrm{t}, J=5.0 \mathrm{~Hz}, 0.5 \mathrm{H}, \mathrm{NCHC} \equiv \mathrm{C}\left(\mathrm{R}^{2}\right)\right.$ ), 4.29 (dd, $J=$ $\left.17.1,4.0 \mathrm{~Hz}, 0.5 \mathrm{H}, \mathrm{NC}(\mathrm{O}) \mathrm{CH}_{2} \mathrm{NHCbz}\left(\mathrm{R}^{2}\right)\right), 4.14$ (dd, $J=18.1,4.2 \mathrm{~Hz}, 0.5 \mathrm{H}, \mathrm{NC}(\mathrm{O}) \mathrm{CH}_{2} \mathrm{NHCbz}\left(\mathrm{R}^{2}\right)$ ), $4.06-3.89\left(\mathrm{~m}, 1 \mathrm{H}, \mathrm{NC}(\mathrm{O}) \mathrm{CH}_{2} \mathrm{NHCbz}\left(\mathrm{R}^{1}\right)\right), 3.74-3.63\left(\mathrm{~m}, 0.5 \mathrm{H}, \mathrm{NCH} \mathrm{H}_{2}\left(\mathrm{CH}_{2}\right)_{2} \mathrm{CHC} \equiv \mathrm{C}\left(\mathrm{R}^{2}\right)\right), 3.61-$ $3.46\left(\mathrm{~m}, 1 \mathrm{H}, \mathrm{NCH}_{2}\left(\mathrm{CH}_{2}\right)_{2} \mathrm{CHC} \equiv \mathrm{C}\left(\mathrm{R}^{1}+\mathrm{R}^{2}\right)\right), 3.44-3.27\left(\mathrm{~m}, 0.5 \mathrm{H}, \mathrm{NCH}_{2}\left(\mathrm{CH}_{2}\right)_{2} \mathrm{CHC} \equiv \mathrm{C}\left(\mathrm{R}^{1}\right)\right), 2.38-$ $1.90\left(\mathrm{~m}, 4 \mathrm{H}, \mathrm{NCH}_{2}\left(\mathrm{CH}_{2}\right)_{2} \mathrm{CHC} \equiv \mathrm{C}\left(\mathrm{R}^{1}+\mathrm{R}^{2}\right)\right) .{ }^{13} \mathrm{C} \mathrm{NMR}(101 \mathrm{MHz}$, Chloroform-d, mixture of rotamers, signals not fully resolved) $\delta 167.0,166.3,156.2,136.4,133.2,133.1,131.6,131.4,128.4,128.0$, 128.0, 123.0, 122.4, 121.6, 120.9, 89.3, 88.0, 83.4, 81.2, 66.8, 48.5, 48.2, 46.1, 45.2, 43.5, 34.4, 32.3, 24.7, 22.8. IR 3285 (w), 2976 (w), 2880 (w), 2361 (w), 2343 (w), 1718 (s), 1654 (s), $1542(\mathrm{~m})$, $1437(\mathrm{~m}), 1436(\mathrm{~m}), 1339(\mathrm{w}), 1260(\mathrm{~m}), 1176(\mathrm{w}), 1070(\mathrm{w}), 1011(\mathrm{w}), 830(\mathrm{w})$. HRMS (ESI) calcd for $\mathrm{C}_{22} \mathrm{H}_{21} \mathrm{BrN}_{2} \mathrm{NaO}_{3}{ }^{+}[\mathrm{M}+\mathrm{Na}]^{+} 463.0628$; found 463.0612 .

\footnotetext{
${ }^{5}$ The quadruplets corresponding to the $\mathrm{CF}_{3}$ couplings were not resolved and appeared as doublets.
} 


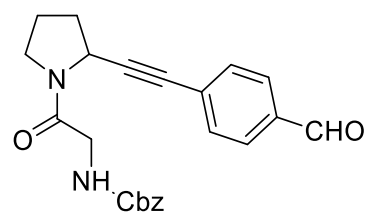

Starting with Cbz-Gly-Pro (5a) (92 mg, $0.30 \mathrm{mmol}, 1.0$ equiv) and pCHO-Ph-EBX (1e) (169 mg, $0.450 \mathrm{mmol}, 1.50$ equiv), $6 \mathrm{~d}$ was obtained after column chromatography DCM to DCM/ethyl acetate $8: 2$ as a yellow oil (105 mg, $0.269 \mathrm{mmol}, 90 \%)$.

$\mathrm{Rf}\left(\mathrm{DCM} /\right.$ ethyl acetate 9:1): 0.33. ${ }^{1} \mathrm{H}$ NMR $\left(400 \mathrm{MHz}\right.$, Chloroform-d, 1:1 mixture of rotamers $\left.\left(\mathrm{R}^{1} / \mathrm{R}^{2}\right)\right)$ $\delta 9.98\left(\mathrm{~s}, 0.5 \mathrm{H}, \mathrm{CHO}\left(\mathrm{R}^{1}\right)\right), 9.96\left(\mathrm{~s}, 0.5 \mathrm{H}, \mathrm{CHO}\left(\mathrm{R}^{2}\right)\right), 7.78\left(\mathrm{dd}, J=14.0,8.0 \mathrm{~Hz}, 2 \mathrm{H}, \mathrm{ArH}\left(\mathrm{R}^{1}+\mathrm{R}^{2}\right)\right)$, $7.53\left(\mathrm{t}, J=7.6 \mathrm{~Hz}, 2 \mathrm{H}, \mathrm{Ar} H\left(\mathrm{R}^{1}+\mathrm{R}^{2}\right)\right), 7.35-7.26\left(\mathrm{~m}, 5 \mathrm{H}, \mathrm{ArH}\left(\mathrm{R}^{1}+\mathrm{R}^{2}\right)\right), 5.81\left(\mathrm{~s}, 1 \mathrm{H}, \mathrm{NH}\left(\mathrm{R}^{1}+\mathrm{R}^{2}\right)\right)$, $5.11\left(\mathrm{~s}, 2 \mathrm{H}, \mathrm{OCH}_{2} \mathrm{Ph}\left(\mathrm{R}^{1}+\mathrm{R}^{2}\right)\right), 5.00\left(\mathrm{~d}, J=4.5 \mathrm{~Hz}, 0.5 \mathrm{H}, \mathrm{NCHC} \equiv \mathrm{C}\left(\mathrm{R}^{1}\right)\right), 4.72(\mathrm{t}, J=5.0 \mathrm{~Hz}, 0.5 \mathrm{H}$, $\left.\mathrm{NCHC} \equiv \mathrm{C}\left(\mathrm{R}^{2}\right)\right), 4.29\left(\mathrm{~d}, J=17.1 \mathrm{~Hz}, 0.5 \mathrm{H}, \mathrm{NC}(\mathrm{O}) \mathrm{CH}_{2} \mathrm{NHCbz}\left(\mathrm{R}^{2}\right)\right), 4.15(\mathrm{~d}, J=17.2 \mathrm{~Hz}, 0.5 \mathrm{H}$, $\left.\mathrm{NC}(\mathrm{O}) \mathrm{CH}_{2} \mathrm{NHCbz}\left(\mathrm{R}^{2}\right)\right), 4.05-3.91\left(\mathrm{~m}, 1 \mathrm{H}, \mathrm{NC}(\mathrm{O}) \mathrm{CH}_{2} \mathrm{NHCbz}\left(\mathrm{R}^{1}\right)\right), 3.76-3.63(\mathrm{~m}, 0.5 \mathrm{H}$, $\left.\mathrm{NCH}_{2}\left(\mathrm{CH}_{2}\right)_{2} \mathrm{CHC} \equiv \mathrm{C}\left(\mathrm{R}^{2}\right)\right), 3.63-3.29\left(\mathrm{~m}, 1.5 \mathrm{H}, \mathrm{NCH}_{2}\left(\mathrm{CH}_{2}\right)_{2} \mathrm{CHC} \equiv \mathrm{C}\left(\mathrm{R}^{1}+\mathrm{R}^{2}\right)\right), 2.35-1.93(\mathrm{~m}, 4 \mathrm{H}$, $\left.\mathrm{NCH}_{2}\left(\mathrm{CH}_{2}\right)_{2} \mathrm{CHC} \equiv \mathrm{C}\left(\mathrm{R}^{1}+\mathrm{R}^{2}\right)\right) .{ }^{13} \mathrm{C} \mathrm{NMR}(101 \mathrm{MHz}$, Chloroform-d, mixture of rotamers, signals not fully resolved) $\delta 191.4,191.3,167.1,166.4,156.2,136.3,135.7,135.3,132.3,132.2,129.4,129.3$, 128.9, 128.4, 128.0, 128.0, 127.9, 92.2, 90.7, 83.5, 81.4, 66.8, 65.7, 48.5, 48.2, 46.1, 45.2, 43.4, 34.3, 32.2, 24.8, 22.8, 15.2. IR $\left(v_{\max }, \mathrm{cm}^{-1}\right) 3654(w), 3401(w), 3321(w), 2982(w), 2883(w), 2729$ (w), $2347(\mathrm{w}), 2230(\mathrm{w}), 1700(\mathrm{~s}), 1657(\mathrm{~s}), 1601(\mathrm{~m}), 1515(\mathrm{~m}), 1435(\mathrm{~s}), 1336(\mathrm{w}), 1262(\mathrm{~m}), 1213$ (m), $1170(\mathrm{~m}), 1053(\mathrm{~m}), 985(\mathrm{w}), 911(\mathrm{w}), 825(\mathrm{~m}), 733$ (s). HRMS (ESI/QTOF) m/z: [M + Na] ${ }^{+}$Calcd for $\mathrm{C}_{23} \mathrm{H}_{22} \mathrm{~N}_{2} \mathrm{NaO}_{4}{ }^{+}$413.1472; Found 413.1478.

\section{Benzyl (2-(2-((4-cyanophenyl)ethynyl)pyrrolidin-1-yl)-2-oxoethyl)carbamate (6e)}

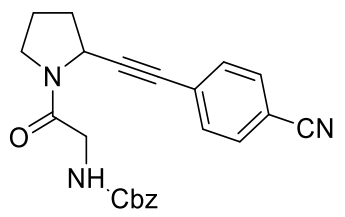

Starting with Cbz-Gly-Pro (5a) (92 mg, $0.30 \mathrm{mmol}, 1.0$ equiv) and pCN-Ph-EBX (1f) (168 mg, 0.450 mmol, 1.50 equiv), 6e was obtained after column chromatography DCM to DCM/ethyl acetate 8:2 as an amorphous solid (108 mg, $0.279 \mathrm{mmol}, 93 \%$ ).

Rf (DCM/ethyl acetate 9:1): 0.33. ${ }^{1} \mathrm{H}$ NMR (400 MHz, Acetonitrile- $d_{3}, 55: 45$ mixture of rotamers $\left.\left(\mathrm{R}^{1} / \mathrm{R}^{2}\right)\right) \delta 7.51-7.41\left(\mathrm{~m}, 2 \mathrm{H}, \mathrm{ArH}\left(\mathrm{R}^{1}+\mathrm{R}^{2}\right)\right), 7.35\left(\mathrm{~d}, J=8.0 \mathrm{~Hz}, 1 \mathrm{H}, \operatorname{Ar} H\left(\mathrm{R}^{1}+\mathrm{R}^{2}\right)\right), 7.28(\mathrm{~d}, J=8.1$ $\left.\mathrm{Hz}, 1 \mathrm{H}, \mathrm{ArH}\left(\mathrm{R}^{1}+\mathrm{R}^{2}\right)\right), 7.17-7.02\left(\mathrm{~m}, 5 \mathrm{H}, \mathrm{ArH}\left(\mathrm{R}^{1}+\mathrm{R}^{2}\right)\right), 5.76-5.54$ (bs, $\left.1 \mathrm{H}, \mathrm{NH}\left(\mathrm{R}^{1}+\mathrm{R}^{2}\right)\right), 4.86$ (s, $\left.2 \mathrm{H}, \mathrm{OCH}_{2} \mathrm{Ph}\left(\mathrm{R}^{1}+\mathrm{R}^{2}\right)\right), 4.70-4.64\left(\mathrm{~m}, 0.55 \mathrm{H}, \mathrm{NCHC} \equiv \mathrm{C}\left(\mathrm{R}^{1}\right)\right), 4.59(\mathrm{dd}, J=7.6,2.4 \mathrm{~Hz}, 0.45 \mathrm{H}$, $\left.\mathrm{NCHC} \equiv \mathrm{C}\left(\mathrm{R}^{2}\right)\right), 4.01\left(\mathrm{dd}, J=17.0,5.8 \mathrm{~Hz}, 0.5 \mathrm{H}, \mathrm{NC}(\mathrm{O}) \mathrm{CH}_{2} \mathrm{NHCbz}\left(\mathrm{R}^{2}\right)\right), 3.78(\mathrm{dd}, J=17.0,5.5 \mathrm{~Hz}$, $\left.0.5 \mathrm{H}, \mathrm{NC}(\mathrm{O}) \mathrm{CH}_{2} \mathrm{NHCbz}\left(\mathrm{R}^{2}\right)\right)$, $3.74-3.56\left(\mathrm{~m}, 1.1 \mathrm{H}, \mathrm{NC}(\mathrm{O}) \mathrm{CH}_{2} \mathrm{NHCbz}\left(\mathrm{R}^{1}\right)\right)$, $3.65(\mathrm{dd}, J=5.5,1.5$ $\left.\mathrm{Hz}, 1.1 \mathrm{H}, \mathrm{NC}(\mathrm{O}) \mathrm{CH}_{2} \mathrm{NHCbz}\left(\mathrm{R}^{1}\right)\right), 3.41-3.26\left(\mathrm{~m}, 1 \mathrm{H}, \mathrm{NCH} \mathrm{CH}_{2}\left(\mathrm{CH}_{2}\right)_{2} \mathrm{CHC} \equiv \mathrm{C}\left(\mathrm{R}^{1}+\mathrm{R}^{2}\right)\right), 3.24-3.11(\mathrm{~m}$, $\left.1 \mathrm{H}, \mathrm{NCH}_{2}\left(\mathrm{CH}_{2}\right)_{2} \mathrm{CHC} \equiv \mathrm{C}\left(\mathrm{R}^{1}+\mathrm{R}^{2}\right)\right), 2.14-1.66\left(\mathrm{~m}, 4 \mathrm{H}, \mathrm{NCH}_{2}\left(\mathrm{CH}_{2}\right)_{2} \mathrm{CHC} \equiv \mathrm{C}\left(\mathrm{R}^{1}+\mathrm{R}^{2}\right)\right) .{ }^{13} \mathrm{C} \mathrm{NMR}(101$ $\mathrm{MHz}$, Acetonitrile- $d_{3}$, mixture of rotamers, signals not fully resolved) $\delta 168.3,167.9,157.5,157.4$, 138.2, 133.3, 133.3, 133.1, 133.0, 129.4, 128.8, 128.7, 128.6, 128.1, 119.3, 119.2, 112.7, 112.3, 94.9, 93.2, 82.9, 80.8, 67.1, 49.2, 48.8, 46.8, 46.1, 44.0, 34.8, 32.9, 25.6, 23.5. IR $\left(\mathrm{V}_{\max }, \mathrm{cm}^{-1}\right) 3616$ (m), 3398 (w), $2918(\mathrm{w}), 2613(\mathrm{w}), 2370(\mathrm{~m}), 2264(\mathrm{~s}), 1728(\mathrm{~s}), 1659(\mathrm{~s}), 1510(\mathrm{~m}), 1453(\mathrm{~m}), 1348$ $(\mathrm{w}), 1254(\mathrm{~m}), 1048(\mathrm{~m}), 843(\mathrm{~m}), 749(\mathrm{w})$. HRMS (ESI/QTOF) m/z: $[\mathrm{M}+\mathrm{Na}]^{+}$Calcd for $\mathrm{C}_{23} \mathrm{H}_{21} \mathrm{~N}_{3} \mathrm{NaO}_{3}{ }^{+} 410.1475$; Found 410.1482 . 


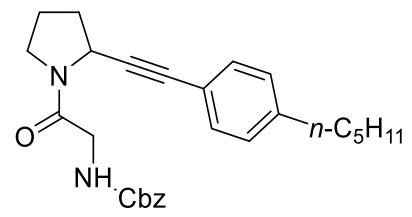

Starting with Cbz-Gly-Pro (5a) (92 mg, 0.30 mmol, 1.0 equiv) and $p C_{5} \mathrm{H}_{11}-\mathrm{Ph}-\mathrm{EBX}(\mathbf{1 g})(168 \mathrm{mg}$, $0.450 \mathrm{mmol}, 1.50$ equiv), $6 \mathrm{f}$ was obtained after column chromatography DCM to DCM/ethyl acetate $8: 2$ as a yellow oil (119 $\mathrm{mg}, 0.275 \mathrm{mmol}, 92 \%)$.

$\mathrm{Rf}$ (DCM/ethyl acetate 9:1): 0.36. ${ }^{1} \mathrm{H}$ NMR (400 MHz, Chloroform-d, 6:4 mixture of rotamers (major/minor)) $\delta 7.40-7.27$ (m, 7H, ArH (major + minor)), 7.09 (dd, $J=13.5,8.0 \mathrm{~Hz}, 2 \mathrm{H}, \mathrm{ArH}$ (major + minor)), $5.93-5.72\left(\mathrm{~m}, 1 \mathrm{H}, \mathrm{NH}\right.$ (major + minor)), $5.13\left(\mathrm{~s}, 2 \mathrm{H}, \mathrm{OCH}_{2} \mathrm{Ph}\right.$ (major+minor)), 5.06 - 4.96 (m, 0.4H, NCHC $\equiv \mathrm{C}$ (minor)), 4.68 (t, $J=4.9 \mathrm{~Hz}, 0.6 \mathrm{H}, \mathrm{NCHC} \equiv \mathrm{C}$ (major)), 4.31 (dd, $J=17.1,2.6 \mathrm{~Hz}$, $0.6 \mathrm{H}, \mathrm{NC}(\mathrm{O}) \mathrm{CH}_{2} \mathrm{NHCbz}$ (major)), 4.18 (dd, $J=17.1,4.0 \mathrm{~Hz}, 0.6 \mathrm{H}, \mathrm{NC}(\mathrm{O}) \mathrm{CH}_{2} \mathrm{NHCbz}$ (major)), 4.07 $-3.87\left(\mathrm{~m}, 0.5 \mathrm{H}, \mathrm{NC}(\mathrm{O}) \mathrm{CH}_{2} \mathrm{NHCbz}\right.$ (minor)), $3.74-3.61\left(\mathrm{~m}, 0.6 \mathrm{H}, \mathrm{NCH}_{2}\left(\mathrm{CH}_{2}\right)_{2} \mathrm{CHC} \equiv \mathrm{C}\right.$ (major)), 3.63 - $3.43\left(\mathrm{~m}, 1 \mathrm{H}, \mathrm{NCH}_{2}\left(\mathrm{CH}_{2}\right)_{2} \mathrm{CHC} \equiv \mathrm{C}\right.$ (major+minor)), $3.44-3.24\left(\mathrm{~m}, 0.4 \mathrm{H}, \mathrm{NCH}_{2}\left(\mathrm{CH}_{2}\right)_{2} \mathrm{CHC} \equiv \mathrm{C}\right.$

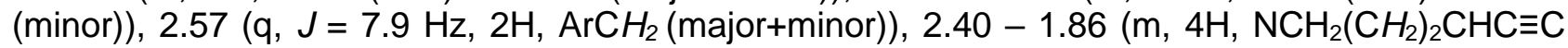
(major+minor)), $1.70-1.49\left(\mathrm{~m}, 2 \mathrm{H}, \mathrm{ArCH}_{2} \mathrm{CH}_{2}\right.$ (major+minor)), $1.44-1.20\left(\mathrm{~m}, 4 \mathrm{H}, \mathrm{CH}_{2} \mathrm{CH}_{2} \mathrm{CH}_{3}\right.$ (major+minor)), $0.97-0.76\left(\mathrm{~m}, 3 \mathrm{H}, \mathrm{CH}_{2} \mathrm{CH}_{3}\right.$ (major+minor)). ${ }^{13} \mathrm{C}$ NMR (101 MHz, Chloroform-d, mixture of rotamers, signals not fully resolved) $\delta 167.1,166.2,156.2,143.8,143.2,136.4,131.6$, 131.5, 128.4, 128.2, 127.9, 127.9, 127.9, 119.7, 119.0, 87.3, 86.1, 84.5, 82.3, 66.7, 48.5, 48.3, 46.0, 45.1, 43.4, 35.7, 35.7, 34.4, 32.4, 31.3, 30.8, 24.6, 22.8, 22.4, 13.9. IR ( $\left.\mathrm{V}_{\max }, \mathrm{cm}^{-1}\right) 3654(\mathrm{w}), 3407$ (m), 3333 (w), 2982 (s), 2926 (s), 2224 (w), 1725 (s), 1657 (s), 1509 (m), 1435 (s), 1336 (w), 1250 (s), $1164(\mathrm{~m}), 1059$ (s), $917(\mathrm{w}), 837(\mathrm{~m}), 745(\mathrm{~m})$. HRMS (ESI/QTOF) m/z: [M + Na] ${ }^{+}$Calcd for $\mathrm{C}_{27} \mathrm{H}_{32} \mathrm{~N}_{2} \mathrm{NaO}_{3}+455.2305$; Found 455.2310.

\section{Benzyl (2-(2-((3-fluorophenyl)ethynyl)pyrrolidin-1-yl)-2-oxoethyl)carbamate (6g)}

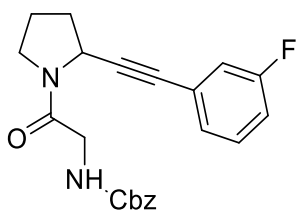

Starting with Cbz-Gly-Pro (5a) (92 mg, 0.30 mmol, 1.0 equiv) and mF-Ph-EBX (1h) (165 mg, 0.450 mmol, 1.50 equiv), $6 \mathrm{~g}$ was obtained after column chromatography DCM to DCM/ethyl acetate 8:2 as a yellow oil (101 mg, $0.266 \mathrm{mmol}, 89 \%$ ).

$\mathrm{Rf}\left(\mathrm{DCM} /\right.$ ethyl acetate 9:1): 0.25. ${ }^{1} \mathrm{H}$ NMR $\left(400 \mathrm{MHz}\right.$, Chloroform- $d, 1: 1$ mixture of rotamers $\left.\left(\mathrm{R}^{1} / \mathrm{R}^{2}\right)\right)$ ס $7.24-6.83\left(\mathrm{~m}, 9 \mathrm{H}, \mathrm{ArH}\left(\mathrm{R}^{1}+\mathrm{R}^{2}\right)\right), 5.74-5.60\left(\mathrm{~m}, 1 \mathrm{H}, \mathrm{NH}\left(\mathrm{R}^{1}+\mathrm{R}^{2}\right)\right), 5.02\left(\mathrm{~s}, 2 \mathrm{H}, \mathrm{OCH} \mathrm{Ph}_{2}\left(\mathrm{R}^{1}+\mathrm{R}^{2}\right)\right)$, $4.89\left(\mathrm{dd}, J=7.3,2.5 \mathrm{~Hz}, 0.5 \mathrm{H}, \mathrm{NCHC} \equiv \mathrm{C}\left(\mathrm{R}^{1}\right)\right), 4.58\left(\mathrm{t}, J=4.9 \mathrm{~Hz}, 0.5 \mathrm{H}, \mathrm{NCHC} \equiv \mathrm{C}\left(\mathrm{R}^{2}\right)\right), 4.19(\mathrm{dd}, J$ $\left.=17.1,4.0 \mathrm{~Hz}, 0.5 \mathrm{H}, \mathrm{NC}(\mathrm{O}) \mathrm{CH}_{2} \mathrm{NHCbz}\left(\mathrm{R}^{2}\right)\right), 4.06\left(\mathrm{dd}, J=17.1,5.1 \mathrm{~Hz}, 0.5 \mathrm{H}, \mathrm{NC}(\mathrm{O}) \mathrm{CH}_{2} \mathrm{NHCbz}\right.$ $\left.\left(\mathrm{R}^{2}\right)\right)$, $3.96-3.80\left(\mathrm{~m}, 1 \mathrm{H}, \mathrm{NC}(\mathrm{O}) \mathrm{CH}_{2} \mathrm{NHCbz}\left(\mathrm{R}^{1}\right)\right)$, $3.66-3.54\left(\mathrm{~m}, 0.5 \mathrm{H}, \mathrm{NCH} \mathrm{H}_{2}\left(\mathrm{CH}_{2}\right)_{2} \mathrm{CHC} \equiv \mathrm{C}\left(\mathrm{R}^{2}\right)\right)$, $3.53-3.35\left(\mathrm{~m}, 1 \mathrm{H}, \mathrm{NCH} \mathrm{CH}_{2}\left(\mathrm{CH}_{2}\right)_{2} \mathrm{CHC} \equiv \mathrm{C}\left(\mathrm{R}^{1}+\mathrm{R}^{2}\right)\right), 3.34-3.20\left(\mathrm{~m}, 0.5 \mathrm{H}, \mathrm{NCH}_{2}\left(\mathrm{CH}_{2}\right)_{2} \mathrm{CHC} \equiv \mathrm{C}\right.$ $\left.\left(\mathrm{R}^{1}+\mathrm{R}^{2}\right)\right), 2.27-1.78\left(\mathrm{~m}, 4 \mathrm{H}, \mathrm{NCH}_{2}\left(\mathrm{CH}_{2}\right)_{2} \mathrm{CHC} \equiv \mathrm{C}\left(\mathrm{R}^{1}+\mathrm{R}^{2}\right)\right) .{ }^{13} \mathrm{C}$ NMR (101 MHz, Chloroform-d, mixture of rotamers, signals not fully resolved) $\delta 167.01,166.3,162.2(\mathrm{~d}, J=246.9 \mathrm{~Hz}), 162.2(\mathrm{~d}, J$ $=246.2 \mathrm{~Hz}), 156.2,136.4,129.9(\mathrm{~d}, J=8.6 \mathrm{~Hz}), 129.7(\mathrm{~d}, J=8.7 \mathrm{~Hz}), 128.4,128.0,128.0,127.9$, $127.7(\mathrm{~d}, J=3.0 \mathrm{~Hz}), 127.6(\mathrm{~d}, J=3.0 \mathrm{~Hz}), 124.5(\mathrm{~d}, J=9.5 \mathrm{~Hz}), 123.7(\mathrm{~d}, J=9.3 \mathrm{~Hz}), 118.7(\mathrm{~d}, J$ $=6.1 \mathrm{~Hz}), 118.4(\mathrm{~d}, J=6.3 \mathrm{~Hz}), 116.1(\mathrm{~d}, J=21.1 \mathrm{~Hz}), 115.5(\mathrm{~d}, J=21.1 \mathrm{~Hz}), 89.1,87.7,83.2(\mathrm{~d}, J$ $=3.5 \mathrm{~Hz}), 81.1(\mathrm{~d}, J=3.1 \mathrm{~Hz}), 66.8,48.5,48.2,46.1,45.2,43.5,34.4,32.3,24.8,22.8 .{ }^{19} \mathrm{~F} \mathrm{NMR}$ (376 MHz, Chloroform-d) $\delta-112.6,-113.2 . I R\left(v_{\max }, \mathrm{cm}^{-1}\right) 3426(\mathrm{~m}), 3308(\mathrm{~m}), 3068(\mathrm{w}), 2976(\mathrm{~m})$, 
$2889(\mathrm{~m}), 2359(\mathrm{w}), 2329(\mathrm{w}), 1719(\mathrm{~s}), 1657(\mathrm{~s}), 1583(\mathrm{~m}), 1527(\mathrm{w}), 1435(\mathrm{~s}), 1343(\mathrm{~m}), 1262(\mathrm{~s})$, $1158(\mathrm{~m}), 1059(\mathrm{~s}), 991(\mathrm{~m}), 868(\mathrm{~m}), 794(\mathrm{~m}), 739(\mathrm{~m})$. HRMS (ESI/QTOF) m/z: [M + Na] ${ }^{+}$Calcd for $\mathrm{C}_{22} \mathrm{H}_{21} \mathrm{FN}_{2} \mathrm{NaO}_{3}{ }^{+}$403.1428; Found 403.1437.

\section{Benzyl (2-(2-((2-bromophenyl)ethynyl)pyrrolidin-1-yl)-2-oxoethyl)carbamate (6h)}

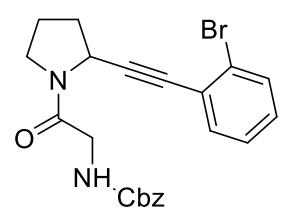

Starting with Cbz-Gly-Pro (5a) (92 mg, $0.30 \mathrm{mmol}, 1.0$ equiv) and o-Br-Ph-EBX (6i) (194 mg, 0.450 mmol, 1.50 equiv), $6 \mathrm{~h}$ was obtained after column chromatography DCM to DCM/ethyl acetate 8:2 as a yellow oil (127 $\mathrm{mg}, 0.290 \mathrm{mmol}, 95 \%)$.

$\mathrm{Rf}$ (DCM/ethyl acetate 9:1): 0.34. ${ }^{1} \mathrm{H}$ NMR $(400 \mathrm{MHz}$, Chloroform- $d$, 6:4 mixture of rotamers (major/minor)) $\delta 7.45$ (ddd, $J=9.1,7.9,1.3 \mathrm{~Hz}, 1 \mathrm{H}, \mathrm{ArH}$ (major+minor)), 7.33 (dd, $J=7.7,1.8 \mathrm{~Hz}$, $1 \mathrm{H}, \mathrm{ArH}$ (major+minor)), $7.29-6.95(\mathrm{~m}, 7 \mathrm{H}, \mathrm{ArH}$ (major+minor)), $5.70(\mathrm{~d}, J=14.9 \mathrm{~Hz}, 1 \mathrm{H}, \mathrm{NH}$ (major+minor)), $5.03\left(\mathrm{~s}, 2 \mathrm{H}, \mathrm{OCH}_{2} \mathrm{Ph}\right.$ (major+minor)), $4.94(\mathrm{~d}, J=6.1 \mathrm{~Hz}, 0.4 \mathrm{H}, \mathrm{NCHC} \equiv \mathrm{C}$ (minor)), $4.63\left(\mathrm{dd}, J=6.6,2.7 \mathrm{~Hz}, 0.6 \mathrm{H}, \mathrm{NCHC} \equiv \mathrm{C}\right.$ (major)), 4.27 (dd, $J=17.2,3.9 \mathrm{~Hz}, 0.6 \mathrm{H}, \mathrm{NC}(\mathrm{O}) \mathrm{CH}_{2} \mathrm{NHCbz}$ (major)), 4.10 (dd, $J=17.2,5.2 \mathrm{~Hz}, 0.6 \mathrm{H}, \mathrm{NC}(\mathrm{O}) \mathrm{CH}_{2} \mathrm{NHCbz}$ (major)), $3.95-3.80$ (m, $0.8 \mathrm{H}$, $\mathrm{NC}(\mathrm{O}) \mathrm{CH}_{2} \mathrm{NHCbz}$ (minor)), $3.67-3.56\left(\mathrm{~m}, 0.6 \mathrm{H}, \mathrm{NCH}_{2}\left(\mathrm{CH}_{2}\right)_{2} \mathrm{CHC} \equiv \mathrm{C}\right.$ (major)), $3.55-3.34(\mathrm{~m}, 1 \mathrm{H}$, $\mathrm{NCH}_{2}\left(\mathrm{CH}_{2}\right)_{2} \mathrm{CHC} \equiv \mathrm{C}$ (minor+major)), $3.35-3.24\left(\mathrm{~m}, 0.4 \mathrm{H}, \mathrm{NCH}_{2}\left(\mathrm{CH}_{2}\right)_{2} \mathrm{CHC} \equiv \mathrm{C}\right.$ (minor)), $2.39-1.78$ (m, $4 \mathrm{H}, \mathrm{NCH}_{2}\left(\mathrm{CH}_{2}\right)_{2} \mathrm{CHC} \equiv \mathrm{C}$ (major+minor)). ${ }^{13} \mathrm{C} \mathrm{NMR}(101 \mathrm{MHz}$, Chloroform-d, mixture of rotamers, signals not fully resolved) $\delta 167.1,166.3,156.2,136.4,136.4,133.4,133.3,132.3,132.2,129.8$, 129.4, 128.4, 128.0, 127.9, 127.0, 126.9, 125.8, 125.8, 124.7, 124.1, 92.9, 91.4, 83.0, 80.9, 66.8, 48.6, 48.3, 46.1, 45.1, 43.5, 43.5, 34.3, 32.3, 29.6, 24.7, 22.8. IR 3411 (w), 3316 (w), 2953 (w), 2880 (w), 2249 (w), $1717(\mathrm{~m}), 1651$ (s), $1511(\mathrm{w}), 1435$ (s), $1341(\mathrm{w}), 1250(\mathrm{~m}), 1175(\mathrm{w}), 1052(\mathrm{~m}), 1027$ (w), 908 (s). HRMS (ESI) calcd for $\mathrm{C}_{22} \mathrm{H}_{22} \mathrm{BrN}_{2} \mathrm{O}_{3}{ }^{+}[\mathrm{M}+\mathrm{H}]^{+} 441.0808$; found 441.0803.

\section{3-Azidopropyl 4-((1-(2-(((benzyloxy)carbonyl)amino)acetyl)pyrrolidin-2-yl)ethynyl)benzoate} (6i)

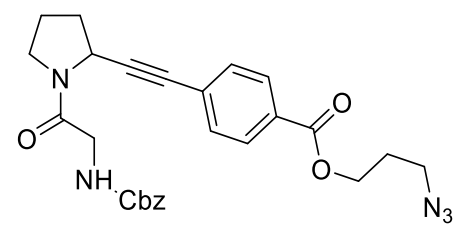

Starting with Cbz-Gly-Pro (5a) (15.3 mg, $0.0500 \mathrm{mmol}, 1.00$ equiv) and $\mathbf{1 j}$ (35.6 mg, $0.0750 \mathrm{mmol}$, 1.50 equiv), $6 \mathbf{i}$ was obtained after preparative TLC DCM/ethyl acetate 9:1 as a yellow oil (16.3 mg $\mathrm{mg}, 0.0330 \mathrm{mmol}, 67 \%)$.

$\mathrm{Rf}\left(\mathrm{DCM} /\right.$ ethyl acetate 9:1): 0.2. ${ }^{1} \mathrm{H}$ NMR $\left(400 \mathrm{MHz}\right.$, Chloroform- $d, 1: 1$ mixture of rotamers $\left.\left(\mathrm{R}^{1} / \mathrm{R}^{2}\right)\right)$ $\delta 8.02-7.91\left(\mathrm{~m}, 2 \mathrm{H}, \mathrm{ArH}\left(\mathrm{R}^{1}+\mathrm{R}^{2}\right)\right), 7.50-7.44\left(\mathrm{~m}, 2 \mathrm{H}, \operatorname{ArH}\left(\mathrm{R}^{1}+\mathrm{R}^{2}\right)\right), 7.40-7.28(\mathrm{~m}, 5 \mathrm{H}, \mathrm{ArH}$ $\left.\left(\mathrm{R}^{1}+\mathrm{R}^{2}\right)\right), 5.75\left(\mathrm{bs}, 1 \mathrm{H}, \mathrm{NH}\left(\mathrm{R}^{1}+\mathrm{R}^{2}\right)\right), 5.13\left(\mathrm{~s}, 2 \mathrm{H}, \mathrm{OCH}_{2} \mathrm{Ph}\left(\mathrm{R}^{1}+\mathrm{R}^{2}\right)\right), 5.02(\mathrm{dd}, J=7.3,2.6 \mathrm{~Hz}, 0.5 \mathrm{H}$, $\left.\mathrm{NCHC} \equiv \mathrm{C}\left(\mathrm{R}^{1}\right)\right), 4.72\left(\mathrm{t}, J=5.0 \mathrm{~Hz}, 0.5 \mathrm{H}, \mathrm{NCHC} \equiv \mathrm{C}\left(\mathrm{R}^{2}\right)\right), 4.41\left(\mathrm{q}, J=6.1 \mathrm{~Hz}, 2 \mathrm{H}, \mathrm{OC} H_{2}\left(\mathrm{R}^{1}+\mathrm{R}^{2}\right)\right)$, 4.30 (dd, $J=17.1,4.0 \mathrm{~Hz}, 0.5 \mathrm{H}, \mathrm{NC}(\mathrm{O}) \mathrm{CH}_{2} \mathrm{NHCbz}\left(\mathrm{R}^{2}\right)$ ), 4.17 (dd, $J=17.1,5.0 \mathrm{~Hz}, 0.5 \mathrm{H}$, $\left.\mathrm{NC}(\mathrm{O}) \mathrm{CH}_{2} \mathrm{NHCbz}\left(\mathrm{R}^{2}\right)\right), 4.05-3.93\left(\mathrm{~m}, 1 \mathrm{H}, \mathrm{NC}(\mathrm{O}) \mathrm{CH}_{2} \mathrm{NHCbz}\left(\mathrm{R}^{1}\right)\right)$, $3.71(\mathrm{ddd}, J=11.5,8.0,3.2$ $\left.\mathrm{Hz}, 0.5 \mathrm{H}, \mathrm{NCH}_{2}\left(\mathrm{CH}_{2}\right)_{2} \mathrm{CHC} \equiv \mathrm{C}\left(\mathrm{R}^{2}\right)\right), 3.61\left(\mathrm{t}, J=9.0 \mathrm{~Hz}, 0.5 \mathrm{H}, \mathrm{NCH}_{2}\left(\mathrm{CH}_{2}\right)_{2} \mathrm{CHC} \equiv \mathrm{C}\left(\mathrm{R}^{1}\right)\right), 3.57-3.35$ $\left(\mathrm{m}, 1 \mathrm{H}, \mathrm{NCH}_{2}\left(\mathrm{CH}_{2}\right)_{2} \mathrm{CHC} \equiv \mathrm{C}\left(\mathrm{R}^{1}+\mathrm{R}^{2}\right)\right), 3.48\left(\mathrm{td}, J=6.7,2.9 \mathrm{~Hz}, 2 \mathrm{H}, \mathrm{N}_{3} \mathrm{CH}_{2}\left(\mathrm{R}^{1}+\mathrm{R}^{2}\right)\right), 2.39-1.92(\mathrm{~m}$, $\left.4 \mathrm{H}, \mathrm{NCH}_{2}\left(\mathrm{CH}_{2}\right)_{2} \mathrm{CHC} \equiv \mathrm{C}\left(\mathrm{R}^{1}+\mathrm{R}^{2}\right)\right), 2.05\left(\mathrm{td}, \mathrm{J}=6.4,3.8 \mathrm{~Hz}, 2 \mathrm{H}, \mathrm{CH}_{2} \mathrm{CH}_{2}\left(\mathrm{R}^{1}+\mathrm{R}^{2}\right)\right) .{ }^{13} \mathrm{C} \mathrm{NMR}(101$ $\mathrm{MHz}$, Chloroform- $d$, mixture of rotamers, signals not fully resolved) $\delta 167.1,166.4,166.4,165.8$, 165.7, 156.2, 136.4, 131.8, 131.7, 129.8, 129.5, 129.3, 129.3, 128.5, 128.1, 128.0, 127.6, 126.8, 
91.3, 89.9, 83.7, 81.6, 66.9, 62.1, 62.0, 48.6, 48.3, 46.2, 45.3, 43.5, 34.4, 32.3, 29.7, 28.2, 24.8, 22.9. IR $\left(v_{\max }, \mathrm{cm}^{-1}\right) 3407(\mathrm{w}), 3333(\mathrm{w}), 2951(\mathrm{w}), 2107(\mathrm{~m}), 1719(\mathrm{~s}), 1657(\mathrm{~s}), 1534(\mathrm{w}), 1447(\mathrm{~m})$, $1275(\mathrm{~s}), 1182(\mathrm{w}), 1102(\mathrm{~m}), 1053(\mathrm{~m}), 862(\mathrm{w}), 776(\mathrm{~m})$. HRMS (ESI/QTOF) m/z: [M + Na] ${ }^{+}$Calcd for $\mathrm{C}_{26} \mathrm{H}_{27} \mathrm{~N}_{5} \mathrm{NaO}_{5}{ }^{+}$512.1904; Found 512.1912.

\section{Prop-2-yn-1-yl 4-((1-(2-(((benzyloxy)carbonyl)amino)acetyl)pyrrolidin-2-yl)ethynyl)benzoate} (6j)

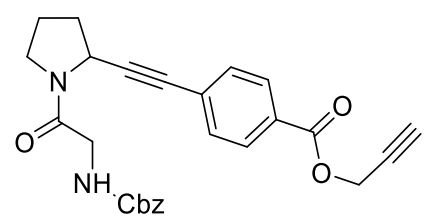

Starting with Cbz-Gly-Pro (5a) (30.6 mg, $0.100 \mathrm{mmol}, 1.00$ equiv) and $1 \mathrm{k}(64.5 \mathrm{mg}, 0.150 \mathrm{mmol}$, 1.50 equiv), 6j was obtained after preparative TLC DCM/ethyl acetate 8:2 as a yellow oil (31.1 mg pure at $95 \%, 0.066 \mathrm{mmol}, 67 \%)$. The isolated product contained traces of alkynylated DMF, a sample for was purified by a second preparative TLC DCM/ethyl acetate 9:1 for characterization.

$\mathrm{Rf}\left(\mathrm{DCM} /\right.$ ethyl acetate 9:1): 0.2. ${ }^{1} \mathrm{H}$ NMR $\left(400 \mathrm{MHz}\right.$, Chloroform- $d, 1: 1$ mixture of rotamers $\left.\left(\mathrm{R}^{1} / \mathrm{R}^{2}\right)\right)$ $\delta 7.99\left(\mathrm{dd}, J=13.1,8.1 \mathrm{~Hz}, 2 \mathrm{H}, \operatorname{Ar} H\left(\mathrm{R}^{1}+\mathrm{R}^{2}\right)\right), 7.60-7.28\left(\mathrm{~m}, 7 \mathrm{H}, \operatorname{Ar} H\left(\mathrm{R}^{1}+\mathrm{R}^{2}\right)\right), 5.88-5.64(\mathrm{~m}$, $\left.1 \mathrm{H}, \mathrm{NH}\left(\mathrm{R}^{1}+\mathrm{R}^{2}\right)\right), 5.12\left(\mathrm{~s}, 2 \mathrm{H}, \mathrm{OCH}_{2} \mathrm{Ph}\left(\mathrm{R}^{1}+\mathrm{R}^{2}\right)\right), 5.02\left(\mathrm{~d}, J=4.6 \mathrm{~Hz}, 0.5 \mathrm{H}, \mathrm{NCHC} \equiv \mathrm{C}\left(\mathrm{R}^{1}\right)\right), 4.92(\mathrm{dd}$, $\left.J=5.6,2.4 \mathrm{~Hz}, 2 \mathrm{H}, \mathrm{CH} \mathrm{H}_{2} \mathrm{C} \equiv \mathrm{C}\left(\mathrm{R}^{1}+\mathrm{R}^{2}\right)\right), 4.72\left(\mathrm{t}, J=4.8 \mathrm{~Hz}, 0.5 \mathrm{H}, \mathrm{NCHC} \equiv \mathrm{C}\left(\mathrm{R}^{2}\right)\right), 4.29(\mathrm{~d}, J=18.3$ $\left.\mathrm{Hz}, 0.5 \mathrm{H}, \mathrm{NC}(\mathrm{O}) \mathrm{CH}_{2} \mathrm{NHCbz}\left(\mathrm{R}^{2}\right)\right), 4.16\left(\mathrm{dd}, J=17.5,3.7 \mathrm{~Hz}, 0.5 \mathrm{H}, \mathrm{NC}(\mathrm{O}) \mathrm{CH}_{2} \mathrm{NHCbz}\left(\mathrm{R}^{2}\right)\right), 4.12-$ $3.92\left(\mathrm{~m}, 1 \mathrm{H}, \mathrm{NC}(\mathrm{O}) \mathrm{CH}_{2} \mathrm{NHCbz}\left(\mathrm{R}^{1}\right)\right), 3.78-3.65\left(\mathrm{~m}, 0.5 \mathrm{H}, \mathrm{NCH}_{2}\left(\mathrm{CH}_{2}\right)_{2} \mathrm{CHC} \equiv \mathrm{C}\left(\mathrm{R}^{2}\right)\right), 3.66-3.46$ $\left(\mathrm{m}, 1 \mathrm{H}, \mathrm{NCH}_{2}\left(\mathrm{CH}_{2}\right)_{2} \mathrm{CHC} \equiv \mathrm{C}\left(\mathrm{R}^{1}+\mathrm{R}^{2}\right)\right), 3.41\left(\mathrm{q}, \mathrm{J}=9.4 \mathrm{~Hz}, 0.5 \mathrm{H}, \mathrm{NCH}_{2}\left(\mathrm{CH}_{2}\right)_{2} \mathrm{CHC} \equiv \mathrm{C}\left(\mathrm{R}^{1}\right)\right), 2.52$ (dt, $\left.J=4.7,2.6 \mathrm{~Hz}, 1 \mathrm{H}, \mathrm{HC} \equiv \mathrm{C}\left(\mathrm{R}^{1}+\mathrm{R}^{2}\right)\right), 2.42-1.90\left(\mathrm{~m}, 4 \mathrm{H}, \mathrm{NCH}_{2}\left(\mathrm{CH}_{2}\right)_{2} \mathrm{CHC} \equiv \mathrm{C}\left(\mathrm{R}^{1}+\mathrm{R}^{2}\right)\right) .{ }^{13} \mathrm{C} \mathrm{NMR}(101$ $\mathrm{MHz}$, Chloroform- $d$, mixture of rotamers, signals not fully resolved) $\delta 167.1,166.4,165.2,165.1,156.2$, $136.4,131.8,131.7,129.7,129.5,129.2,128.7,128.5,128.1,128.0,128.0,127.9,127.0,91.5,90.0,83.6$, 81.5, 75.2, 75.1, 66.9, 52.6, 52.6, 48.6, 48.3, 46.2, 45.3, 43.5, 34.4, 32.3, 24.8, 22.9. IR ( $\left.\mathrm{v}_{\max }, \mathrm{cm}^{-1}\right) 3660$ (w), $3407(\mathrm{w}), 3271(\mathrm{w}), 2982(\mathrm{~m}), 1725(\mathrm{~s}), 1651(\mathrm{~m}), 1515(\mathrm{w}), 1429(\mathrm{~m}), 1256(\mathrm{~s}), 1102(\mathrm{~s}), 1059$ (s), $1010(\mathrm{~m}), 862(\mathrm{~m}), 751(\mathrm{~m}), 745(\mathrm{~m})$. HRMS (ESI/QTOF) m/z: [M + Na] ${ }^{+}$Calcd for $\mathrm{C}_{26} \mathrm{H}_{24} \mathrm{~N}_{2} \mathrm{NaO}_{5}{ }^{+}$ 467.1577; Found 467.1569.

\section{(E)-Benzyl (2-oxo-2-(2-styrylpyrrolidin-1-yl)ethyl)carbamate (8)}

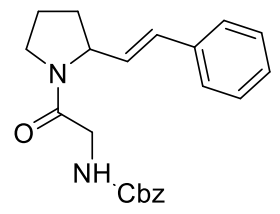

Starting with Cbz-Gly-Pro (5a) (92 mg, 0.30 mmol, 1.0 equiv) and Ph-VBX (34) (158 mg, $0.450 \mathrm{mmol}$, 1.50 equiv), 8 was obtained after column chromatography DCM to DCM/ethyl acetate 8:2 as a yellow oil (89.5 mg, $0.246 \mathrm{mmol}, 82 \%)$.

$\mathrm{Rf}$ (DCM/ethyl acetate 9:1): 0.17. ${ }^{1} \mathrm{H}$ NMR $(400 \mathrm{MHz}$, Chloroform-d, 6:4 mixture of rotamers (major/minor)) $\delta 7.44-7.23(\mathrm{~m}, 10 \mathrm{H}, \mathrm{ArH}$ (major+minor)), $6.42(\mathrm{dd}, J=15.8,7.7 \mathrm{~Hz}, 1 \mathrm{H},=\mathrm{CHPh}$ (major+minor)), 6.10 (dd, $J=15.9,6.1 \mathrm{~Hz}, 1 \mathrm{H}, \mathrm{NCHCH}=$ (major+minor)), $5.79(\mathrm{~s}, 0.4 \mathrm{H}, \mathrm{NH}$ (minor), $5.73\left(\mathrm{~s}, 0.6 \mathrm{H}, \mathrm{NH}\right.$ (major), $5.12\left(\mathrm{~s}, 0.8 \mathrm{H}, \mathrm{OCH}_{2} \mathrm{Ph}\right.$ (minor)), 5.09 (s, 1.2H, OCH $\mathrm{OHh}_{2}$ (major)), 4.83 (t, $J=6.3 \mathrm{~Hz}, 0.4 \mathrm{H}, \mathrm{NCHC} \equiv \mathrm{C}$ (minor)), $4.50(\mathrm{t}, J=7.0 \mathrm{~Hz}, 0.6 \mathrm{H}, \mathrm{NCHC} \equiv \mathrm{C}$ (major)), $4.17-3.84$ (m, $2 \mathrm{H}, \mathrm{NC}(\mathrm{O}) \mathrm{CH}_{2} \mathrm{NHCbz}$ (major+minor)), $3.71-3.36\left(\mathrm{~m}, 2 \mathrm{H}, \mathrm{NCH}_{2}\left(\mathrm{CH}_{2}\right)_{2}\right.$ (major+minor)), 2.27 - 1.83 (m, $\left.4 \mathrm{H}, \mathrm{NCH}_{2}\left(\mathrm{CH}_{2}\right)_{2} \mathrm{CHC} \equiv \mathrm{C}\right) .{ }^{13} \mathrm{C}$ NMR $(101 \mathrm{MHz}$, Chloroform-d, mixture of rotamers, signals not fully resolved) $\delta 167.3,166.5,156.2,156.1,136.5,136.4,135.8,133.6,130.6,130.2,128.7,128.7$, 128.6, 128.4, 128.3, 128.1, 128.0, 128.0, 127.9, 127.5, 126.5, 126.4, 126.4, 123.9, 123.2, 66.8, 58.8, 
46.5, 45.7, 43.5, 43.4, 33.2, 30.7, 23.8, 21.6. IR $\left(\mathrm{v}_{\max }, \mathrm{cm}^{-1}\right) 3666(\mathrm{w}), 3401(\mathrm{w}), 3308(\mathrm{w}), 2982(\mathrm{~s})$, $2902(\mathrm{~m}), 2341$ (w), 2187 (w), 1731 (s), 1657 (s), $1509(\mathrm{~m}), 1441(\mathrm{~m}), 1262(\mathrm{~m}), 1164(\mathrm{w}), 1065(\mathrm{~s})$, $905(\mathrm{w}), 751(\mathrm{~m})$. HRMS (ESI/QTOF) m/z: [M + Na] ${ }^{+}$Calcd for $\mathrm{C}_{22} \mathrm{H}_{24} \mathrm{~N}_{2} \mathrm{NaO}_{3}{ }^{+}$387.1679; Found 387.1684.

\section{Benzyl ((2S)-1-oxo-3-phenyl-1-(2-(phenylethynyl)pyrrolidin-1-yl)propan-2-yl)carbamate (9a)}

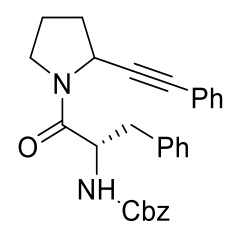

Starting with Cbz-Phe-Pro (5b) (119 $\mathrm{mg}, 0.300 \mathrm{mmol})$, the crude product was purified by column chromatography DCM to DCM/ethyl acetate $8: 2$ to afford 9 a as two separable diastereoisomers (ratio 2:1 by RP-HPLC analysis and NMR of the crude mixture): a pale yellow oil $(59.7 \mathrm{mg}, 0.132$ mmol, $44 \%$, major) as fraction 1 and an amorphous solid (47.0 mg, $0.104 \mathrm{mmol}, 35 \%$, minor) as fraction 2 .

$\operatorname{Rf}(\mathrm{DCM} /$ ethyl acetate 9:1): 0.44 (fraction 1); 0.32 (fraction 2).

Fraction 1 (major):

${ }^{1} \mathrm{H}$ NMR (400 MHz, Chloroform- $d, 6: 4$ mixture of rotamers (major/minor)) $\delta 7.52-7.02(\mathrm{~m}, 15 \mathrm{H}, \mathrm{ArH}$ (major+minor)), $5.81-5.62\left(\mathrm{~m}, 1 \mathrm{H}, \mathrm{NH}\right.$ (major+minor)), $5.13-5.03\left(\mathrm{~m}, 2.4 \mathrm{H}, \mathrm{OCH}_{2} \mathrm{Ph}\right.$ (major+minor) and $\mathrm{NC}(\mathrm{O}) \mathrm{CHN}$ (minor)), 4.98 (dd, $J=6.0,4.0 \mathrm{~Hz}, 0.6 \mathrm{H}, \mathrm{NCHC} \equiv \mathrm{C}$ (major)), 4.62 (td, $J=8.5,5.5 \mathrm{~Hz}, 0.6 \mathrm{H}, \mathrm{NC}(\mathrm{O}) \mathrm{CHN}$ (major)), $3.77-3.68(\mathrm{~m}, 0.4 \mathrm{H}, \mathrm{NCHC} \equiv \mathrm{C}$ (minor)), $3.67-3.57(\mathrm{~m}$, $0.6 \mathrm{H}, \mathrm{NCH}_{2}$ (major)), $3.43-3.22\left(\mathrm{~m}, 1 \mathrm{H}, \mathrm{NCH}_{2}\right.$ (major+minor)), 3.20 (dd, $J=12.7,4.9 \mathrm{~Hz}, 0.4 \mathrm{H}$, $\mathrm{CH}_{2} \mathrm{Ph}$ (minor)), $3.13-3.01\left(\mathrm{~m}, 1.2 \mathrm{H}, \mathrm{CH}_{2} \mathrm{Ph}\right.$ (major+minor)), 2.95 (dd, $J=12.7,10.0 \mathrm{~Hz}, 0.4 \mathrm{H}$, $\mathrm{CH}_{2} \mathrm{Ph}$ (minor)), $2.80-2.69\left(\mathrm{~m}, 0.4 \mathrm{H}, \mathrm{NCH}_{2}\right.$ (minor)), $2.06-1.51\left(\mathrm{~m}, 4 \mathrm{H}, \mathrm{NCH}_{2}\left(\mathrm{CH}_{2}\right)_{2} \mathrm{CHC} \equiv \mathrm{C}\right.$ (major+minor)). ${ }^{13} \mathrm{C}$ NMR (101 MHz, Chloroform- $d$, mixture of rotamers, signals not fully resolved) $\delta$ $169.9,169.5,155.6,155.1,136.4,136.4,135.8,131.9,131.7,129.8,129.5,128.5,128.5,128.4$, $128.4,128.2,128.2,128.1,128.0,127.9,127.0,126.9,123.0,122.2,88.2,87.0,84.2,82.3,66.8$, 66.6, 54.4, 53.8, 48.7, 48.3, 46.0, 45.6, 41.1, 40.0, 33.8, 32.2, 24.8, 22.7. IR $\left(\mathrm{v}_{\max }, \mathrm{cm}^{-1}\right) 3284(\mathrm{~m})$, 3031 (w), 2963 (w), 2359 (w), 2335 (w), 1712 (s), 1632 (s), $1534(\mathrm{~m}), 1435$ (s), 1336 (m), 1262 (s), $1041(\mathrm{~m}), 763(\mathrm{~m})$. HRMS (ESI/QTOF) m/z: $[\mathrm{M}+\mathrm{Na}]^{+}$Calcd for $\mathrm{C}_{29} \mathrm{H}_{28} \mathrm{~N}_{2} \mathrm{NaO}_{3}{ }^{+}$475.1992; Found 475.2008.

Fraction 2 (minor):

${ }^{1} \mathrm{H}$ NMR (400 MHz, Chloroform-d, 6:4 mixture of rotamers (major/minor)) $\delta 7.45-7.09(\mathrm{~m}, 15 \mathrm{H}, \mathrm{Ar} H$ (major+minor)), $5.83-5.71(\mathrm{~m}, 0.4 \mathrm{H}, \mathrm{NH}$ (minor)), 5.46 (bs, $0.6 \mathrm{H}, \mathrm{NH}$ (major)), 5.25 (dd, $J=5.9$, $3.7 \mathrm{~Hz}, 0.6 \mathrm{H}, \mathrm{NCHC} \equiv \mathrm{C}$ (major)), $5.15-4.95\left(\mathrm{~m}, 2.4 \mathrm{H}, \mathrm{OCH}_{2} \mathrm{Ph}\right.$ (major+minor) + $\mathrm{NC}(\mathrm{O}) \mathrm{CHN}$ (major)), 4.82 (dd, $J=7.8,2.0 \mathrm{~Hz}, 0.4 \mathrm{H}, \mathrm{NCHC} \equiv \mathrm{C}$ (minor)), $4.67(\mathrm{td}, J=9.0,5.7 \mathrm{~Hz}, 0.4 \mathrm{H}$, $\mathrm{NC}(\mathrm{O}) \mathrm{C} H \mathrm{~N}$ (major)), $3.72-3.47\left(\mathrm{~m}, 1.6 \mathrm{H}, \mathrm{NCH}_{2}\right.$ (major+minor)), $3.32(\mathrm{dd}, J=14.1,4.1 \mathrm{~Hz}, 0.6 \mathrm{H}$, $\mathrm{CH}_{2} \mathrm{Ph}$ (major)), $3.13-2.91$ (m, 1.4H, $\mathrm{CH}_{2} \mathrm{Ph}$ (major+minor)), 2.54 (td, $\mathrm{J}=9.2,6.9 \mathrm{~Hz}, 0.4 \mathrm{H}, \mathrm{NCH}$ (minor)), $2.36-1.89\left(\mathrm{~m}, 3.6 \mathrm{H}, \mathrm{NCH}_{2}\left(\mathrm{CH}_{2}\right)_{2}\right), 1.63$ (dtd, $\left.J=9.7,6.7,3.5 \mathrm{~Hz}, 0.4 \mathrm{H}, \mathrm{NCH}_{2}\left(\mathrm{CH}_{2}\right)_{2}\right) .{ }^{13} \mathrm{C}$ NMR (101 MHz, Chloroform- $d$, mixture of rotamers, signals not fully resolved) $\delta 171.0,169.3,156.0$, 155.5, 136.6, 136.3, 136.2, 131.8, 131.6, 129.5, 129.3, 128.6, 128.4, 128.4, 128.3, 128.0, 128.0, 128.0, 127.9, 127.8, 127.0, 126.6, 122.8, 122.1, 88.4, 88.2, 84.2, 82.1, 66.7, 54.1, 53.7, 49.3, 48.5, 46.1, 45.9, 40.3, 38.3, 34.5, 32.3, 24.3, 23.1. HRMS (ESI/QTOF) $\mathrm{m} / \mathrm{z}:[\mathrm{M}+\mathrm{Na}]^{+}$Calcd for $\mathrm{C}_{29} \mathrm{H}_{28} \mathrm{~N}_{2} \mathrm{NaO}_{3}+475.1992 ;$ Found 475.1994 .

Benzyl (2-((1,4-diphenylbut-3-yn-2-yl)amino)-2-oxoethyl)carbamate (9b)

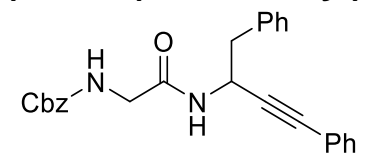


Starting with Cbz-Gly-Phe $(5 \mathrm{c})(107 \mathrm{mg}, 0.300 \mathrm{mmol})$, the crude product was purified by column chromatography DCM to DCM/ethyl acetate $9: 1$ to afford $9 \mathrm{~b}$ as a pale yellow solid (93 $\mathrm{mg}, 0.23$ $\mathrm{mmol}, 68 \%)$.

$\mathrm{Rf}\left(\mathrm{DCM} /\right.$ ethyl acetate 9:1): 0.36. Mp 128.9 - 130.8 ${ }^{\circ} \mathrm{C}$. ${ }^{1} \mathrm{H}$ NMR $(400 \mathrm{MHz} \text {, Chloroform- } d)^{6} \delta 7.33$ $(\mathrm{m}, 15 \mathrm{H}, \mathrm{ArH}), 6.15(\mathrm{~d}, J=8.4 \mathrm{~Hz}, 1 \mathrm{H}, \mathrm{NH}), 5.36(\mathrm{bs}, 1 \mathrm{H}, \mathrm{NH}), 5.29-5.20(\mathrm{~m}, 1 \mathrm{H}, \mathrm{NCHC} \equiv \mathrm{C}), 5.13$ (s, $2 \mathrm{H}, \mathrm{OCH}_{2} \mathrm{Ph}$ ), $3.95-3.77\left(\mathrm{~m}, 2 \mathrm{H}, \mathrm{NC}(\mathrm{O}) \mathrm{CH}_{2} \mathrm{NHCbz}\right), 3.07\left(\mathrm{~m}, 2 \mathrm{H}, \mathrm{Ph}-\mathrm{CH}_{2}\right) .{ }^{13} \mathrm{C} \mathrm{NMR}(101 \mathrm{MHz}$, Chloroform- $d)^{6} \delta 168.7,167.7,136.1,136.1,131.7,130.0,128.6,128.6,128.4,128.3,128.2,127.1$, 122.3, 87.2, 84.7, 67.4, 43.1, 41.5, 29.7. IR 3316 (w), 2988 (w), 2881 (w), 2361 (s), 2342 (s), 2125 (w), 2086 (w), 1772 (w), 1683 (s), 1542 (s), 1397 (w), 1267 (m), 1138 (w). HRMS (ESI) calcd for $\mathrm{C}_{26} \mathrm{H}_{24} \mathrm{~N}_{2} \mathrm{NaO}_{3}{ }^{+}\left[\mathrm{M}^{+} \mathrm{Na}\right]^{+} 435.1679$; found 435.1682 .

\section{Benzyl (1-oxo-1-((4-phenylbut-3-yn-2-yl)amino)propan-2-yl)carbamate (9c)}

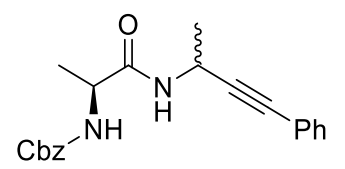

Starting with Cbz-Ala-Ala (5d) (88 $\mathrm{mg}, 0.30 \mathrm{mmol}, 1.0$ equiv), 9c was obtained after column chromatography DCM to DCM/ethyl acetate $8: 2$ as a white solid ( $83 \mathrm{mg}, 0.24 \mathrm{mmol}, 79 \%$ ) and as a mixture of unresolved diastereoisomers.

Rf (DCM/ethyl acetate 95:5): 0.28. Mp $171-172{ }^{\circ} \mathrm{C} .{ }^{1} \mathrm{H}$ NMR $(400 \mathrm{MHz}$, Chloroform- $d) \delta 7.45-$ $7.28(\mathrm{~m}, 10 \mathrm{H}, \mathrm{ArH}), 6.32$ (bs, $1 \mathrm{H}, \mathrm{NH}), 5.30$ (bs, $1 \mathrm{H}, \mathrm{NH}), 5.12\left(\mathrm{~s}, 2 \mathrm{H}, \mathrm{OCH}_{2} \mathrm{Ph}\right), 5.07-4.94(\mathrm{~m}, 1 \mathrm{H}$, $\mathrm{NCHC} \equiv \mathrm{C}), 4.24(\mathrm{~m}, 1 \mathrm{H}, \mathrm{CCHNHCbz}), 1.46\left(\mathrm{~d}, J=6.9 \mathrm{~Hz}, 3 \mathrm{H}, \mathrm{CH}_{3} \mathrm{CHC} \equiv \mathrm{C}\right), 1.40$ (d, J=7.0 Hz, 3H, $\mathrm{CH}_{3} \mathrm{CHNHCbz}$ ). ${ }^{13} \mathrm{C}$ NMR (101 MHz, Chloroform- $d$ ) $\delta$ 170.9, 156.0, 136.0, 131.7, 128.6, 128.4, 128.3, 128.1, 128.1, 122.5, 88.9, 82.5, 67.1, 50.5, 37.8, 37.8, 22.4. IR $3294(\mathrm{~m}), 3067(\mathrm{w}), 3035(\mathrm{w})$, $2984(\mathrm{w}), 2361$ (w), 1708 (s), $1656(\mathrm{~s}), 1540(\mathrm{~s}), 1456(\mathrm{~m}), 1330(\mathrm{w}), 1257(\mathrm{~m}), 1241(\mathrm{~m}), 1135(\mathrm{w})$, $1071(\mathrm{~m}), 1029$ (w), $956(\mathrm{w}), 915(\mathrm{w})$. HRMS (ESI) calcd for $\mathrm{C}_{21} \mathrm{H}_{23} \mathrm{~N}_{2} \mathrm{O}_{3}{ }^{+}[\mathrm{M}+\mathrm{H}]^{+} 351.1703$; found 351.1697.

\section{(S)-Benzyl 2-((3-phenylprop-2-yn-1-yl)carbamoyl)pyrrolidine-1-carboxylate (9d)}

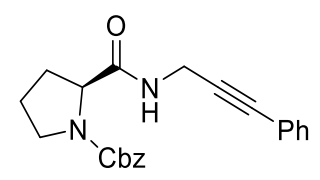

Starting with Cbz-Pro-Gly (5e) $(92 \mathrm{mg}, 0.30 \mathrm{mmol})$, and using 4CICzIPN (9.58 mg, $900 \mu \mathrm{mol}, 3.00$ mol\%) as catalyst, the crude product was purified by column chromatography DCM to DCM/ethyl acetate $85: 15$ to afford $9 \mathbf{d}$ as a pale yellow solid $(63.4 \mathrm{mg}, 0.175 \mathrm{mmol}, 58 \%)$.

Rf (DCM/ethyl acetate 9:1): 0.36. Mp $124.7-126.1^{\circ} \mathrm{C} .{ }^{1} \mathrm{H}$ NMR $\left(400 \mathrm{MHz}\right.$, Acetonitrile- $d_{3}$, mixture of rotamers, signals not fully resolved) $\delta 7.18-6.88(\mathrm{~m}, 10 \mathrm{H}, \operatorname{ArH}), 6.79(\mathrm{~d}, J=18.0 \mathrm{~Hz}, 1 \mathrm{H}, \mathrm{NH})$, $4.83-4.65\left(\mathrm{~m}, 2 \mathrm{H}, \mathrm{OCH} \mathrm{Ph}_{2}\right), 3.88(\mathrm{dd}, J=8.8,3.5 \mathrm{~Hz}, 1 \mathrm{H}, \mathrm{NCH}), 3.79$ (dd, $J=14.1,5.7 \mathrm{~Hz}, 2 \mathrm{H}$, $\left.\mathrm{CH}_{2} \mathrm{C} \equiv \mathrm{C}\right), 3.26-3.04\left(\mathrm{~m}, 2 \mathrm{H}, \mathrm{NCH}_{2}\right), 1.61$ (p, $\left.J=2.5 \mathrm{~Hz}, 2 \mathrm{H}, \mathrm{NCH}_{2} \mathrm{CH}_{2}\right), 1.53(\mathrm{q}, J=6.9 \mathrm{~Hz}, 2 \mathrm{H}$, $\mathrm{NCHCH}_{2}$ ). ${ }^{13} \mathrm{C}$ NMR (101 MHz, Acetonitrile- $d_{3}$, mixture of rotamers, signals not fully resolved) $\delta$ 173.4, 173.0, 138.1, 132.4, 129.4, 129.4, 129.3, 128.8, 128.6, 128.3, 123.6, 86.8, 82.6, 67.5, 67.4, $61.8,61.5,48.1,47.7,32.0,30.6,29.8,25.0,24.2$.

IR $\left(v_{\max }, \mathrm{cm}^{-1}\right) 3678(\mathrm{w}), 3302(\mathrm{w}), 2982(\mathrm{~s}), 2889(\mathrm{~m}), 2359(\mathrm{w}), 1700(\mathrm{~s}), 1534(\mathrm{~m}), 1417(\mathrm{~s}), 1349$ $(\mathrm{m}), 1244(\mathrm{~m}), 1195(\mathrm{w}), 1115(\mathrm{~m}), 1090(\mathrm{~m}), 924(\mathrm{w}), 757$ (s). HRMS (ESI/QTOF) m/z: [M + Na] Calcd for $\mathrm{C}_{22} \mathrm{H}_{22} \mathrm{~N}_{2} \mathrm{NaO}_{3}{ }^{+}$385.1523; Found 385.1519.

${ }^{6}$ One carbon is not resolved. 


\subsection{Peptide tetramers scope}

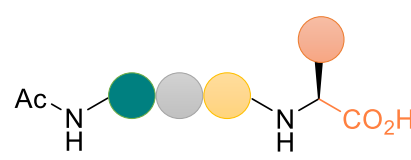

10

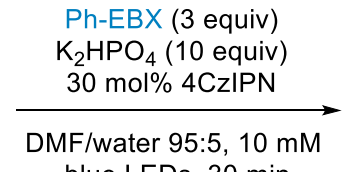

blue LEDs, $30 \mathrm{~min}$

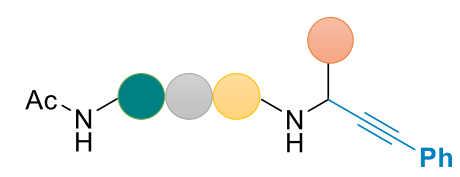

11

\section{General procedure 4 for the decarboxylative alkynylation of tetramers}

A $20 \mathrm{mM}$ solution of the tetramer in non-degassed DMF (50 $\mu \mathrm{L}, 1.0 \mu \mathrm{mol})$, a $60 \mu \mathrm{M}$ solution of PhEBX (1b) in DMF (25 $\mu \mathrm{L}, 3.0 \mu \mathrm{mol}, 3.0$ equiv), a $15 \mu \mathrm{M}$ solution of $4 \mathrm{CzIPN}(\mathbf{2 a})$ in DMF $(20 \mu \mathrm{L}, 0.30$ $\mu \mathrm{mol}, 30 \mathrm{~mol} \%$ ) and a $2 \mathrm{M}$ solution of $\mathrm{K}_{2} \mathrm{HPO}_{4}$ in milli-Q purified water ( $5 \mu \mathrm{L}, 10 \mu \mathrm{mol}, 10$ equiv) were placed into a vial. The vial was then caped and degassed by bubbling with argon for 1-2 min and the mixture was irradiated using blue light LEDs for 30 min at RT.

Intermediate samples were prepared by dilution of $10 \mu \mathrm{L}$ of the reaction mixture with $30 \mu \mathrm{L}$ of $\mathrm{MeOH}$. At the end of the reaction, the crude was diluted with $3 x$ the volume of MeCN/water 1:1 or with $3 x$ the volume of MeCN/water 3:1 and injected in RP-HPLC (volume of injection adapted to the dilution). The yields were determined as the ratio of $A_{\text {prod }} / A_{\text {total }}$ where $A_{\text {prod }}=$ area in $m A U$ of the product peak and $A_{\text {total }}=$ area in $\mathrm{mAU}$ of all peptides products (product, starting material, and side-products if present).

Reported results are an average of a minimum of 3 independent trials. The products are described as peptide- $\mathrm{AH}$.

\section{Reference HPLC-UV chromatograms of reagents at $214 \mathrm{~nm}$}
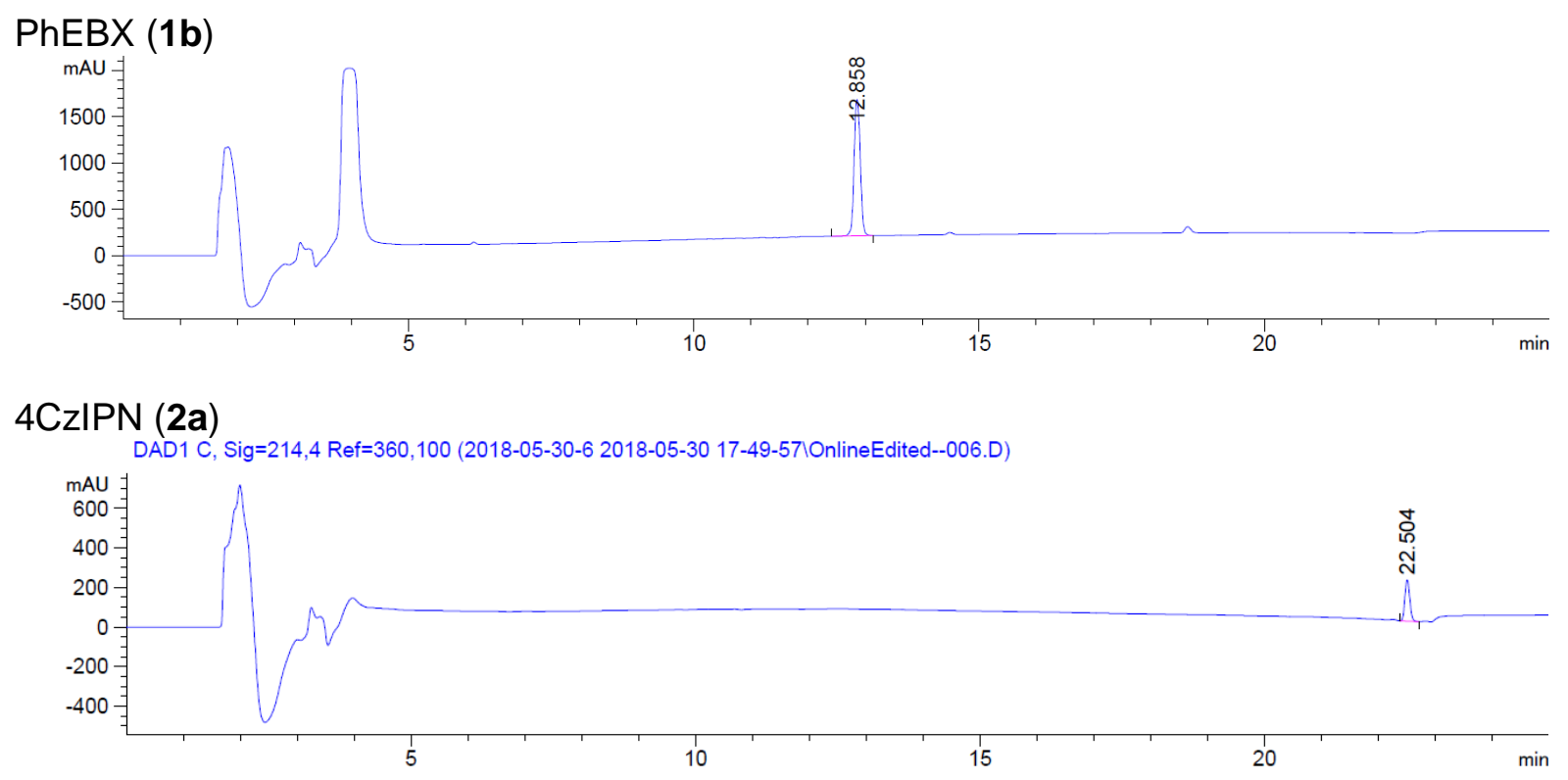

$\left\{\operatorname{lr}\left[\mathrm{dF}\left(\mathrm{CF}_{3}\right) \mathrm{ppy}_{2} \mathrm{dtbbpy}\right\} \mathrm{PF}_{6}(\mathbf{4})\right.$ 


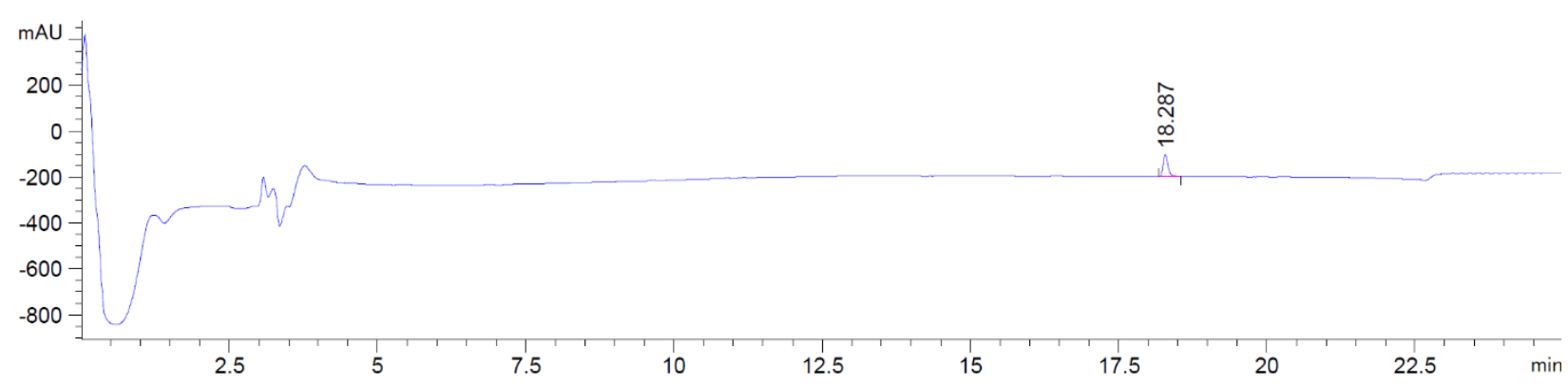

lodobenzoic acid (14)

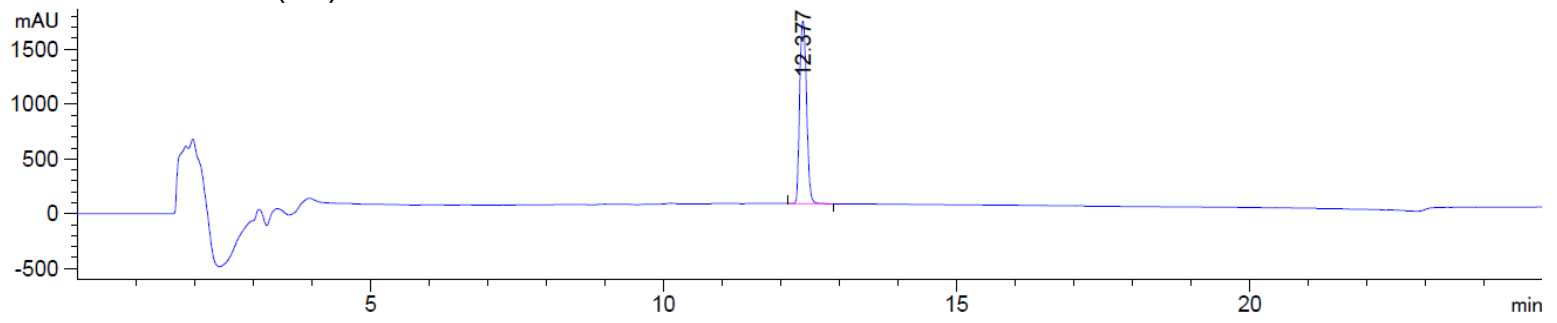

Control experiment without peptide

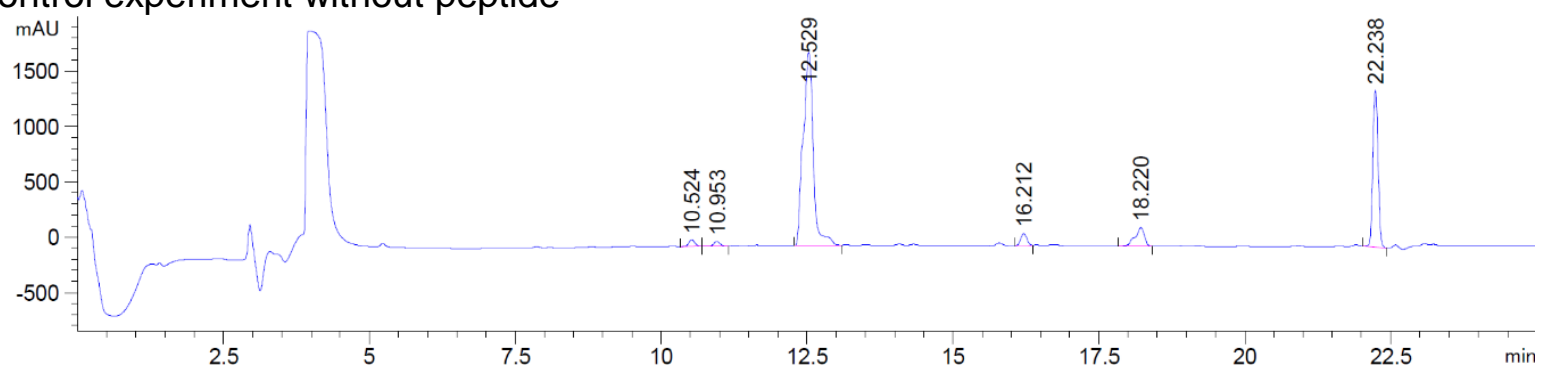

For clarity in the reaction chromatograms, the peaks of peptide-based products are indicated by an arrow. In all reactions, iodobenzoic acid, Ph-EBX and the catalyst were present, together with side products at $10.524,16.212$ and $18.220 \mathrm{~min}$. The alkyne dimer and alkynylated DMF were identified by NMR but low ionization did not allow confirmation of their retention time. 
<smiles>CC(=O)N[C@@H](C)C(=O)N[C@@H](Cc1ccccc1)C(=O)NCC(=O)N1CCCC1C#Cc1ccccc1</smiles>

Following the general procedure, Ac-Ala-Phe-Gly-Pro-OH (10a) afforded 11a in in more than 95\% yield (retention time 13.330).

HRMS (ESI/QTOF) m/z: [M + Na] ${ }^{+}$Calcd for $\mathrm{C}_{28} \mathrm{H}_{32} \mathrm{~N}_{4} \mathrm{NaO}_{4}{ }^{+} 511.2316$; Found 511.2315.

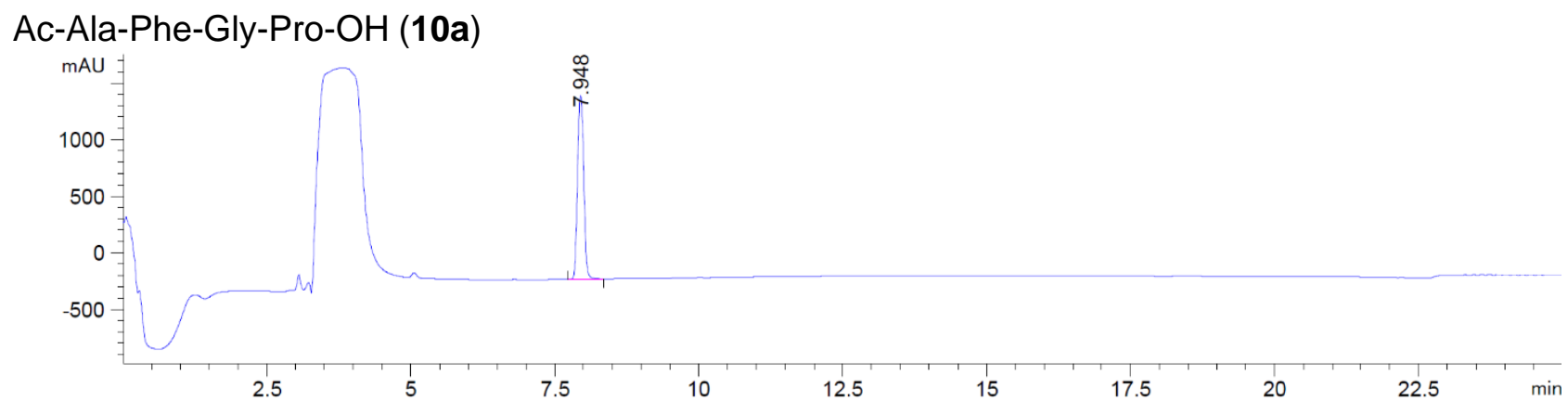

HRMS of 10a (ESI/QTOF) m/z: [M + $\left.\mathrm{H}_{-1}\right]^{-}$Calcd for $\mathrm{C}_{21} \mathrm{H}_{27} \mathrm{~N}_{4} \mathrm{O}_{6}{ }^{-} 431.1936$; Found 431.1938.

HPLC-UV chromatogram at $214 \mathrm{~nm}$ of the crude reaction mixture

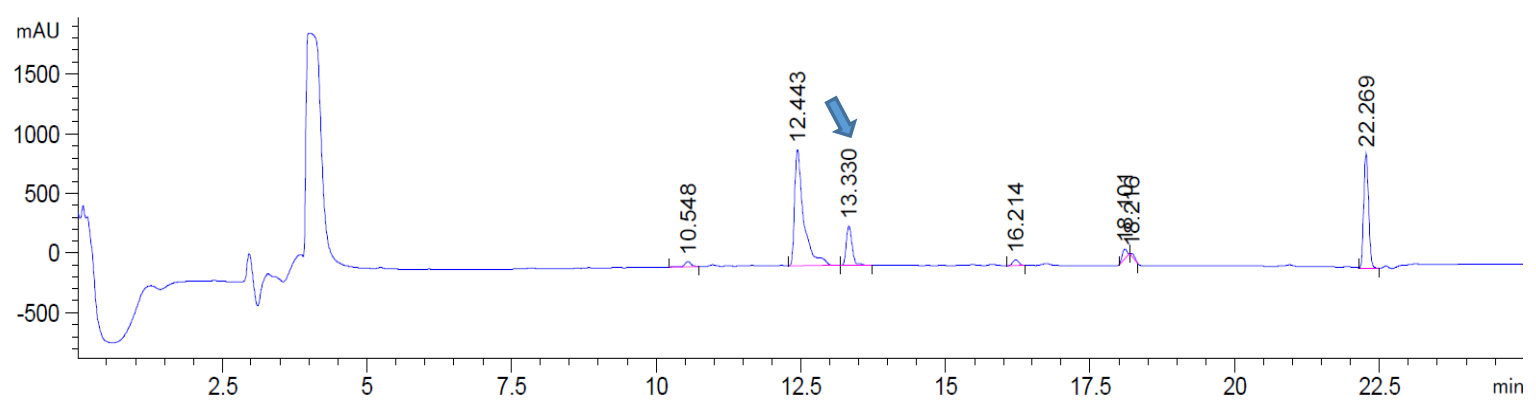

\section{Ac-Arg-Phe-Gly-Pro-AH (11b)}<smiles>CC(=O)N[C@@H](CCCNC(=N)N)C(=O)N[C@@H](Cc1ccccc1)C(=O)NCC(=O)N1CCCC1C#Cc1ccccc1</smiles>

Following the general procedure, Ac-Arg-Phe-Gly-Pro-OH (10b) afforded 11b in in more than 95\% yield (retention time 10.573). 
HRMS (ESI/QTOF) m/z: [M] ${ }^{+}$Calcd for $\mathrm{C}_{31} \mathrm{H}_{40} \mathrm{~N}_{7} \mathrm{O}_{4}{ }^{+}$574.3136; Found 574.3151.

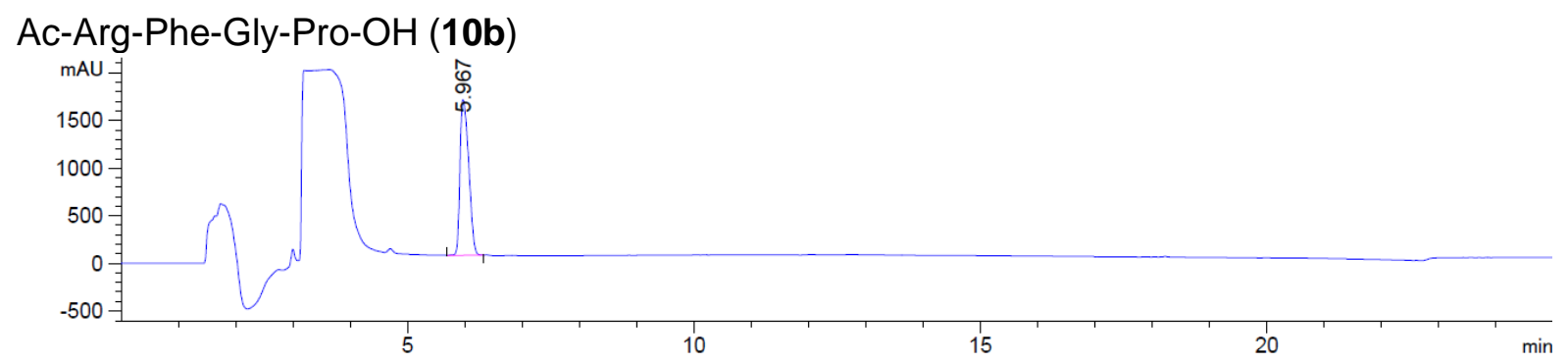

HRMS of 10b (ESI/QTOF) m/z: [M + $\left.\mathrm{H}_{-1}\right]^{-}$Calcd for $\mathrm{C}_{24} \mathrm{H}_{34} \mathrm{~N}_{7} \mathrm{O}_{6}{ }^{-}$516.2576; Found 516.2579.

HPLC-UV chromatogram at $214 \mathrm{~nm}$ of the crude reaction mixture

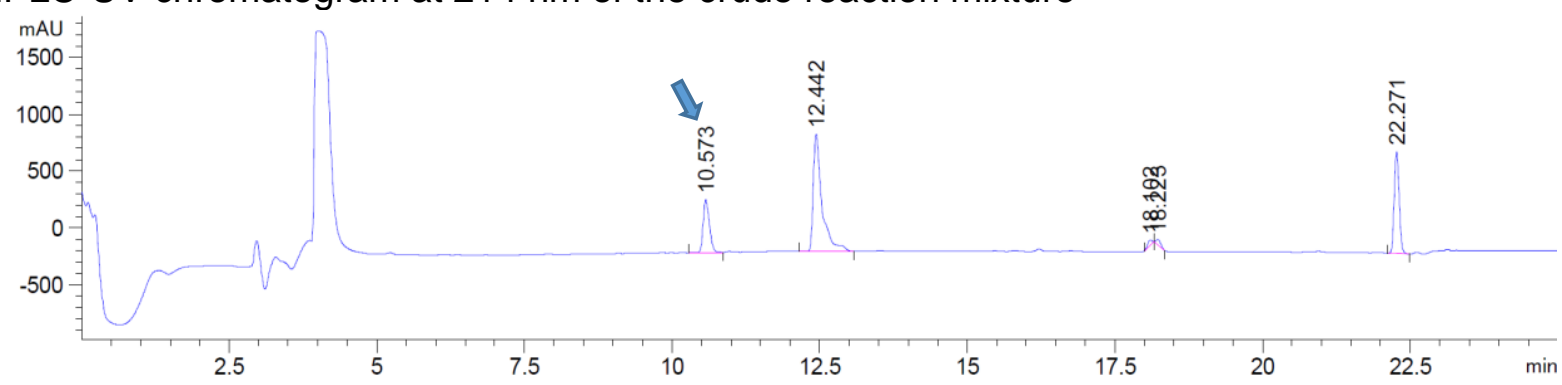

Ac-His-Phe-Gly-Pro-AH (11c)<smiles></smiles>

Following the general procedure, Ac-His-Phe-Gly-Pro-OH (10c) afforded 11c in $84 \%$ yield (retention time 10.778 with method A, 17.054 with method B for a better separation).

HRMS (ESI/QTOF) m/z: [M + H] ${ }^{+}$Calcd for $\mathrm{C}_{31} \mathrm{H}_{35} \mathrm{~N}_{6} \mathrm{O}_{4}{ }^{+}$555.2714; Found 555.2722.

Ac-His-Phe-Gly-Pro-OH (10c)

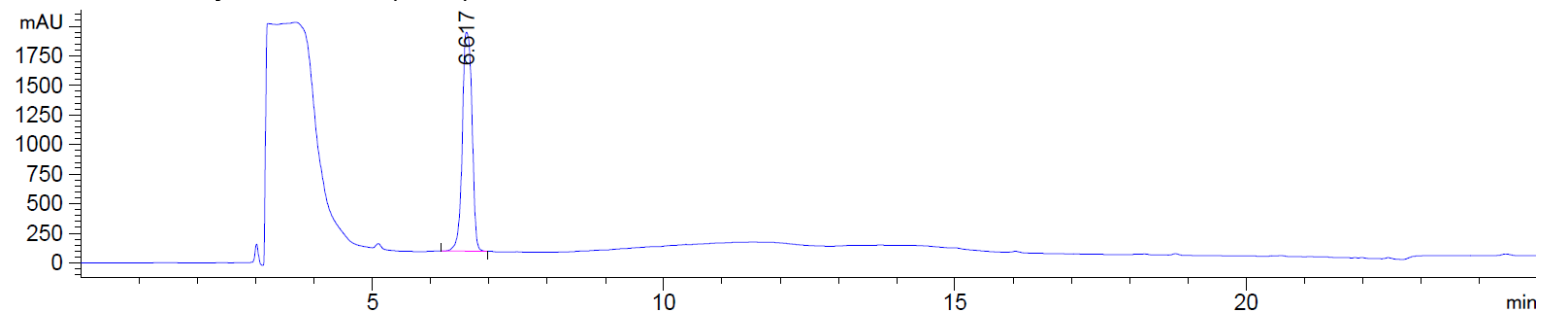

HRMS of 10c (ESI/QTOF) m/z: [M + $\left.\mathrm{H}_{-1}\right]^{-}$Calcd for $\mathrm{C}_{24} \mathrm{H}_{29} \mathrm{~N}_{6} \mathrm{O}_{6}{ }^{-}$497.2154; Found 497.2147.

HPLC-UV chromatogram at $214 \mathrm{~nm}$ of the crude reaction mixture (method $\mathrm{A}$ ) 


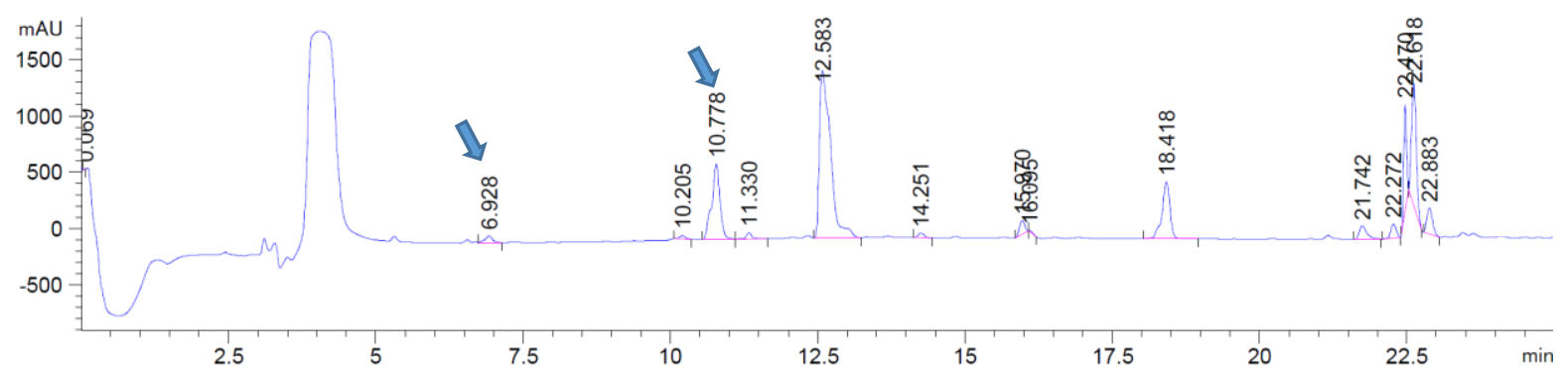

HPLC-UV chromatogram at $214 \mathrm{~nm}$ of the crude reaction mixture (method B)

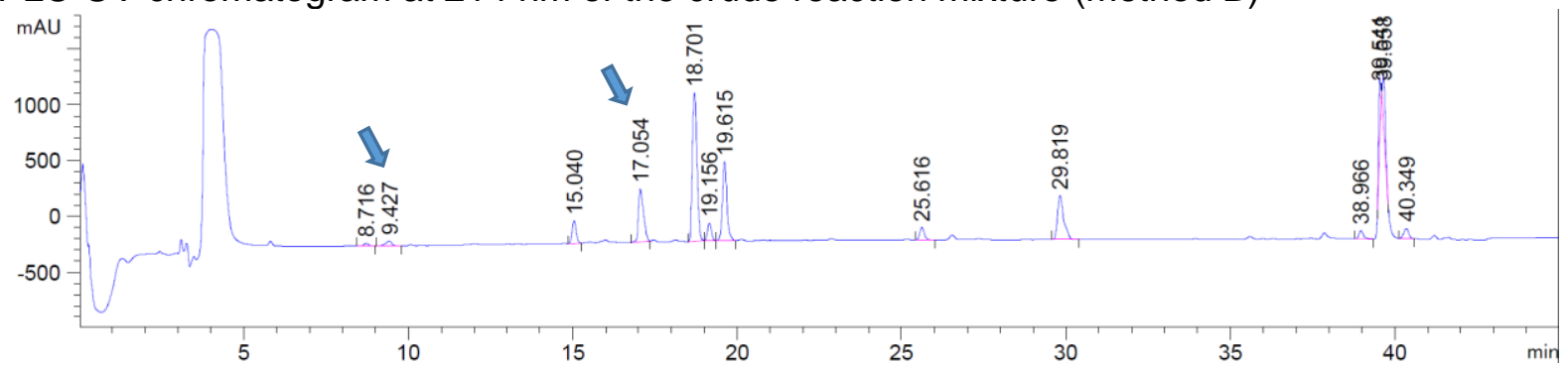

\section{Ac-Lys-Phe-Gly-Pro-AH (11d)}

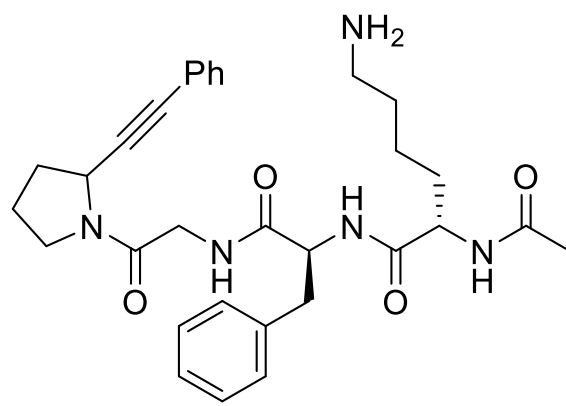

Following the general procedure, Ac-Lys-Phe-Gly-Pro-OH (10d) afforded 11d in 47\% yield (retention time 10.670).

HRMS (ESI/QTOF) m/z: [M] ${ }^{+}$Calcd for $\mathrm{C}_{31} \mathrm{H}_{40} \mathrm{~N}_{5} \mathrm{O}_{4}{ }^{+}$546.3075; Found 546.3071.

Ac-Lys-Phe-Gly-Pro-OH (10d)

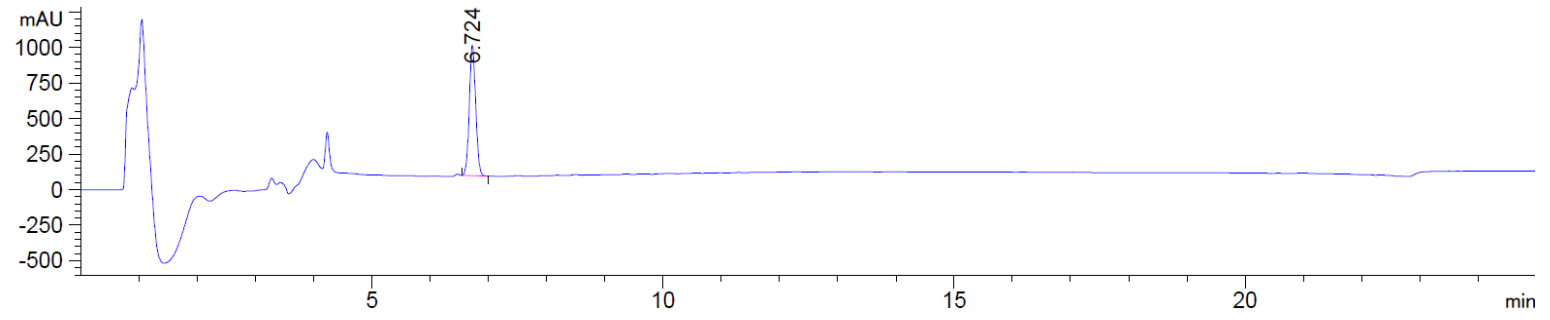

HRMS of 10d (ESI/QTOF) m/z: [M + $\left.\mathrm{H}_{-1}\right]^{-}$Calcd for $\mathrm{C}_{24} \mathrm{H}_{34} \mathrm{~N}_{5} \mathrm{O}_{6}{ }^{-}$488.2515; Found 488.2518.

HPLC-UV chromatogram at $214 \mathrm{~nm}$ of the crude reaction mixture 


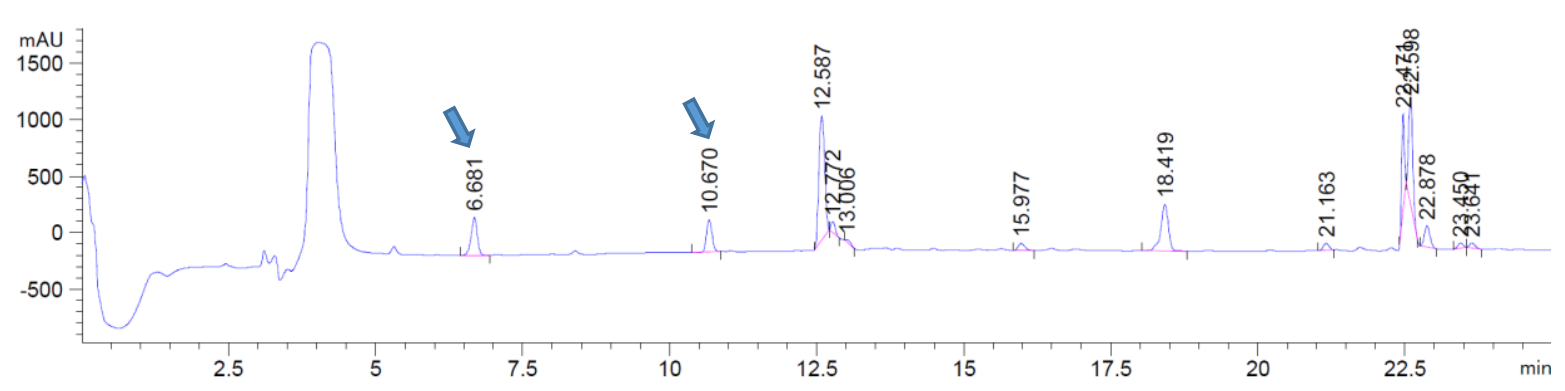

Ac-Tyr-Phe-Gly-Pro-AH (11e)<smiles>CC(=O)N[C@@H](Cc1ccc(O)cc1)C(=O)N[C@@H](Cc1ccccc1)C(=O)NCC(=O)N1CCCC1C#Cc1ccccc1</smiles>

Following the general procedure and using 4tBuCzIPN (2c) as catalyst, Ac-Tyr-Phe-Gly-Pro-OH (10e) afforded 11e in $20 \%$ yield (retention time 13.942).

Following the general procedure and using $\operatorname{Ir}(\mathbf{4})$ as catalyst, Ac-Tyr-Phe-Gly-Pro-OH (10e) afforded 11 in $54 \%$ yield (retention time 13.937).

HRMS (ESI/QTOF) m/z: [M + Na] ${ }^{+}$Calcd for $\mathrm{C}_{34} \mathrm{H}_{36} \mathrm{~N}_{4} \mathrm{NaO}_{5}{ }^{+}$603.2578; Found 603.2580.

Ac-Tyr-Phe-Gly-Pro-OH (10e)

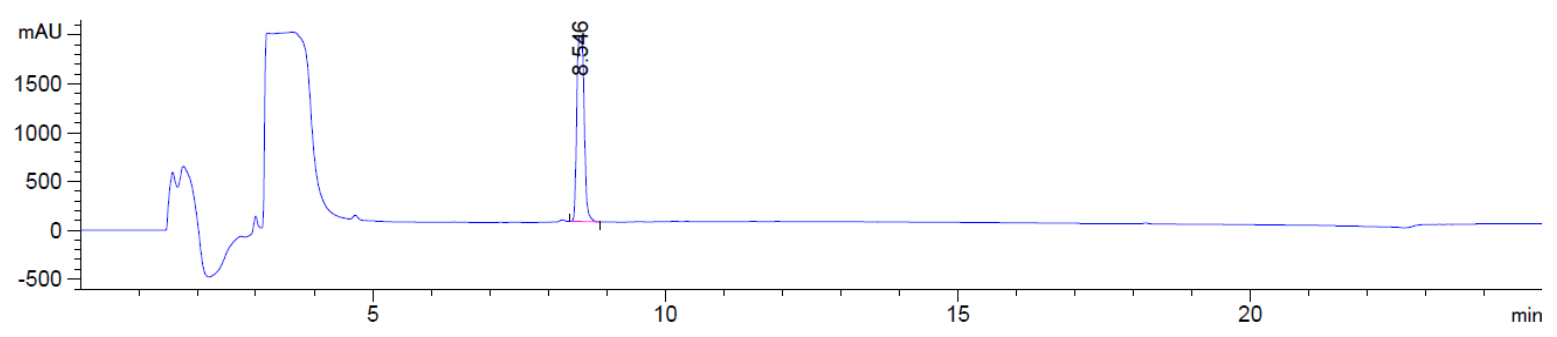

HRMS of 10e (ESI/QTOF) m/z: [M + $\left.\mathrm{H}_{-1}\right]^{-}$Calcd for $\mathrm{C}_{27} \mathrm{H}_{31} \mathrm{~N}_{4} \mathrm{O}_{7}-523.2198$; Found 523.2205.

4 tBuCzIPN (2c) as the catalyst

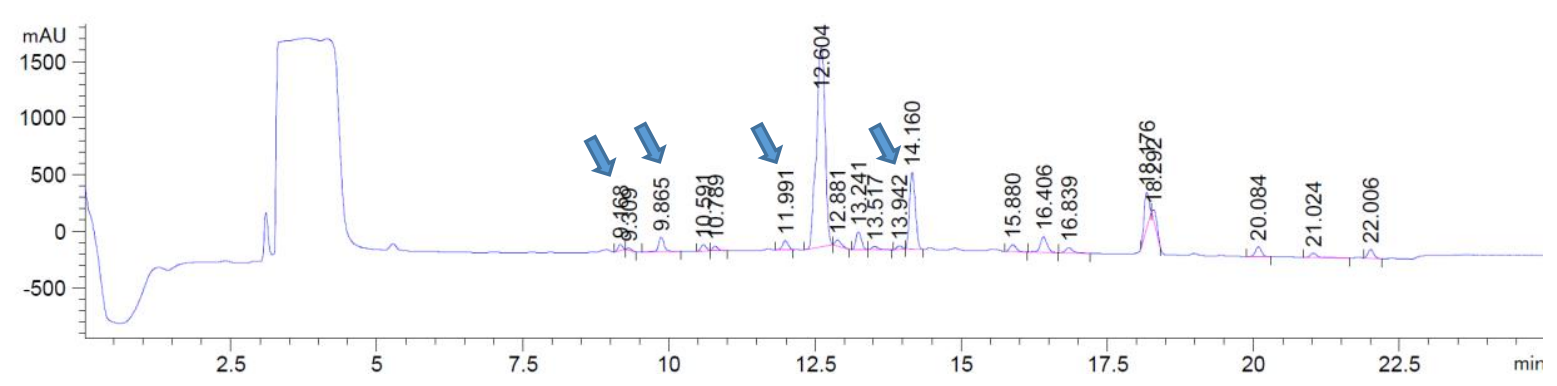

$\left\{\operatorname{Ir}\left[\mathrm{dF}\left(\mathrm{CF}_{3}\right) \mathrm{ppy}\right]_{2} \mathrm{dtbbpy}_{\mathrm{P}} \mathrm{PF}_{6}(\mathbf{4})\right.$ as the catalyst 


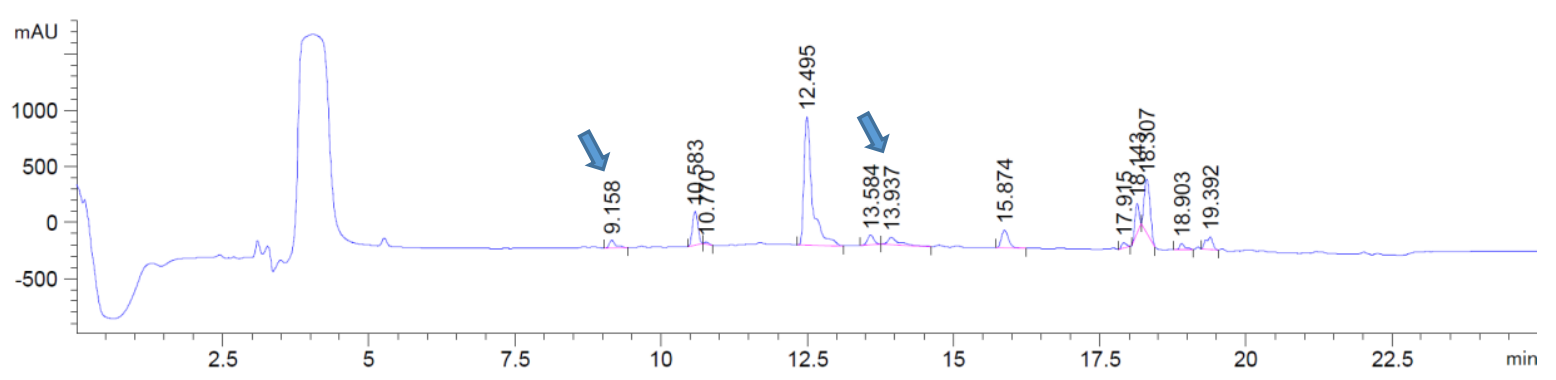

Ac-Trp-Phe-Gly-Pro-AH (11f)

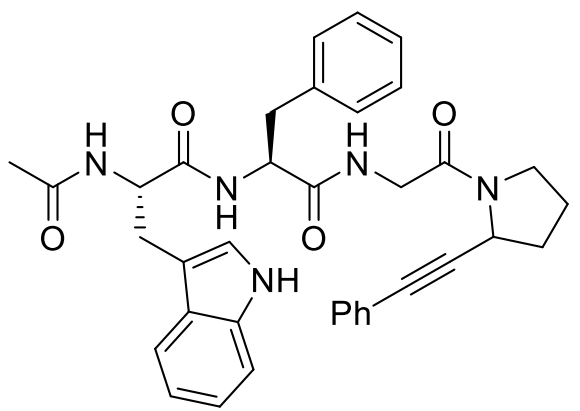

Following the general procedure, no desired product was detected from Ac-Trp-Phe-Gly-Pro-OH (10f) neither with 4CzIPN (2a), 4tBuCzIPN (2c) nor Ir cat (4).

Ac-Trp-Phe-Gly-Pro-OH (10f)

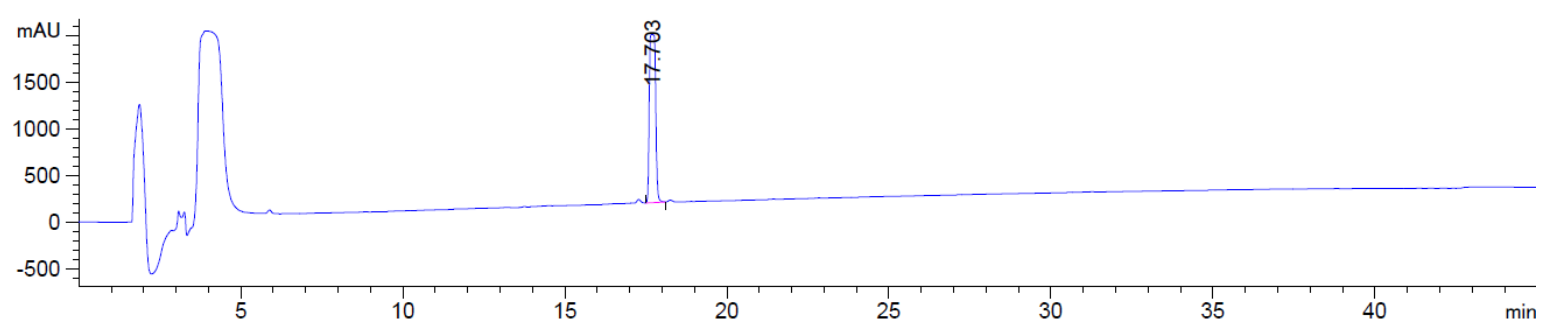

HRMS of 10 (ESI/QTOF) m/z: [M + $\left.\mathrm{H}_{-1}\right]^{-}$Calcd for $\mathrm{C}_{29} \mathrm{H}_{32} \mathrm{~N}_{5} \mathrm{O}_{6}{ }^{-}$546.2358; Found 546.2357.

\section{Ac-Trp(Boc)-Phe-Gly-Pro-AH (11f')}

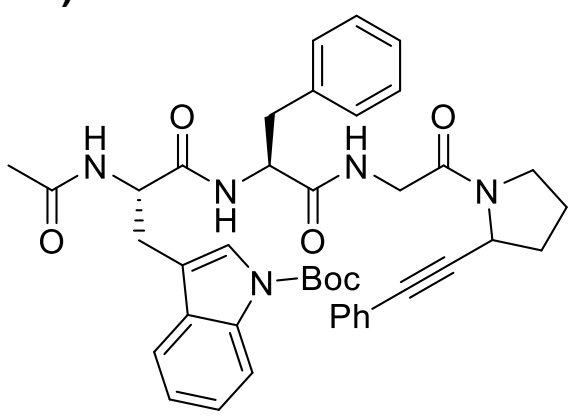

Following the general procedure, Ac-Trp(Boc)-Phe-Gly-Pro-OH (10f') afforded 11f' in 89\% yield (retention time 18.973).

HRMS (nanochip-ESI/LTQ-Orbitrap) $\mathrm{m} / \mathrm{z}$ : $[\mathrm{M}+\mathrm{H}]^{+}$Calcd for $\mathrm{C}_{41} \mathrm{H}_{46} \mathrm{~N}_{5} \mathrm{O}_{6}+704.3443$; Found 704.3460. 
Ac-Trp(Boc)-Phe-Gly-Pro-OH (10f)

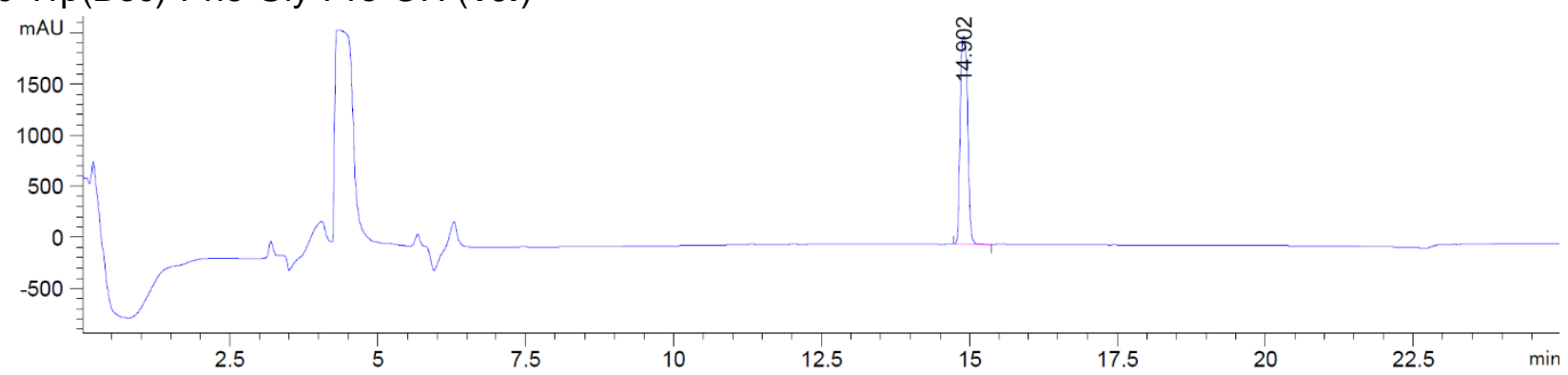

HRMS of 10f' (nanochip-ESI/LTQ-Orbitrap) m/z: [M + H] $]^{+}$Calcd for $\mathrm{C}_{34} \mathrm{H}_{42} \mathrm{~N}_{5} \mathrm{O}_{8}{ }^{+}$648.3028; Found 648.3050 .

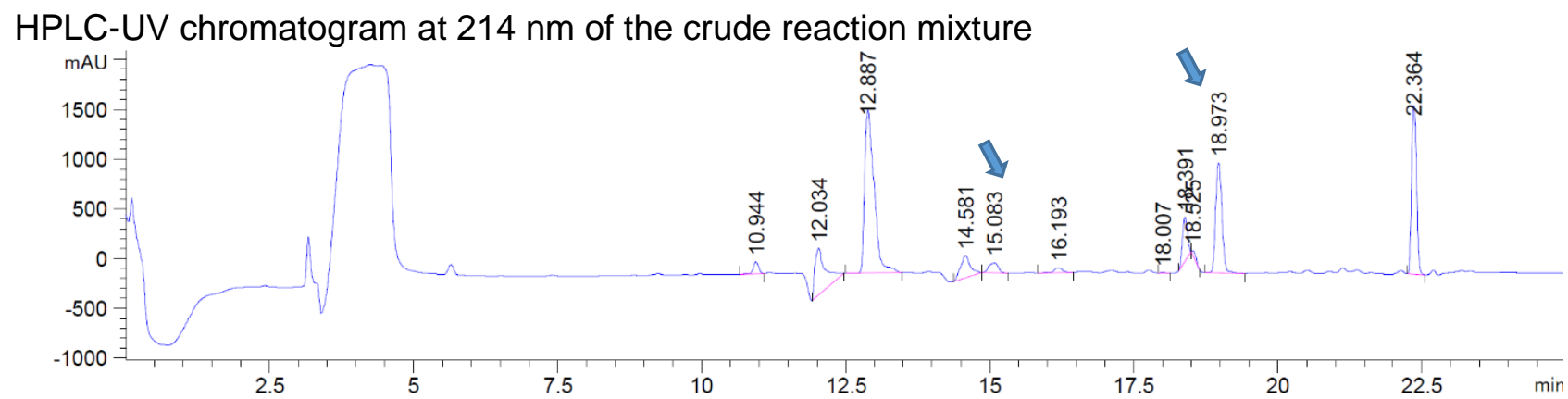

\section{Ac-Cys-Phe-Gly-Pro-AH (11g)}<smiles>CC(=O)N[C@@H](CSC#Cc1ccccc1)C(=O)N[C@@H](Cc1ccccc1)C(=O)NCC(=O)N1CCCC1C#Cc1ccccc1</smiles>

Following the general procedure, Ac-Cys-Phe-Gly-Pro-OH (10g) afforded $\mathbf{1 1 g}$ in more than 95\% yield (retention time 17.432).

HRMS (QTOF) m/z: [M + K]+ Calcd for $\mathrm{C}_{36} \mathrm{H}_{36} \mathrm{KN}_{4} \mathrm{O}_{4} \mathrm{~S}^{+}$659.2089; Found 659.2091.

Ac-Cys-Phe-Gly-Pro-OH (10g)

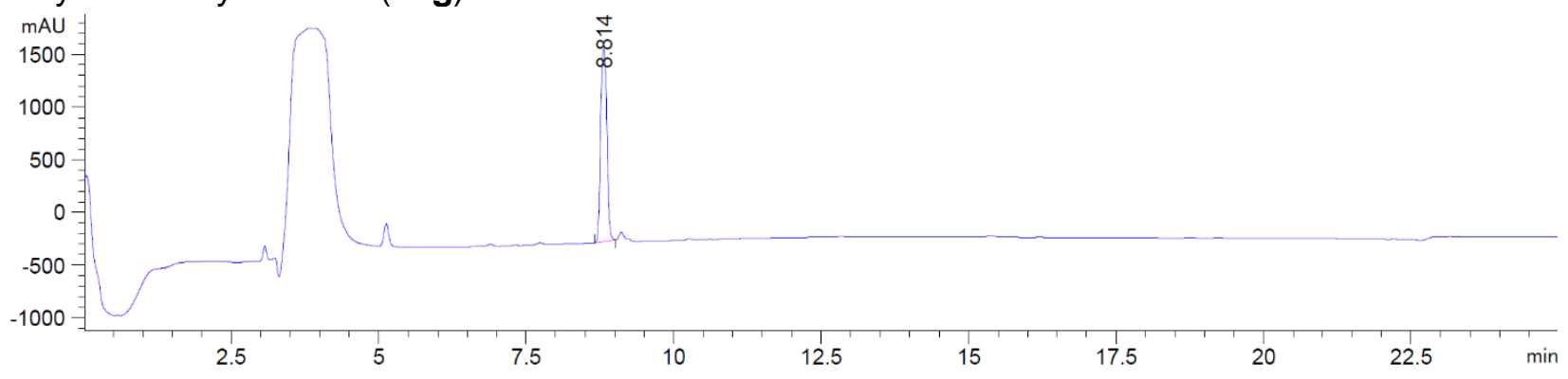

HRMS of $10 \mathrm{~g}$ (ESI/QTOF) m/z: [M + Na] $]^{+}$Calcd for $\mathrm{C}_{21} \mathrm{H}_{28} \mathrm{~N}_{4} \mathrm{NaO}_{6} \mathrm{~S}^{+}$487.1622; Found 487.1632. 
HPLC-UV chromatogram at $214 \mathrm{~nm}$ of the crude reaction mixture

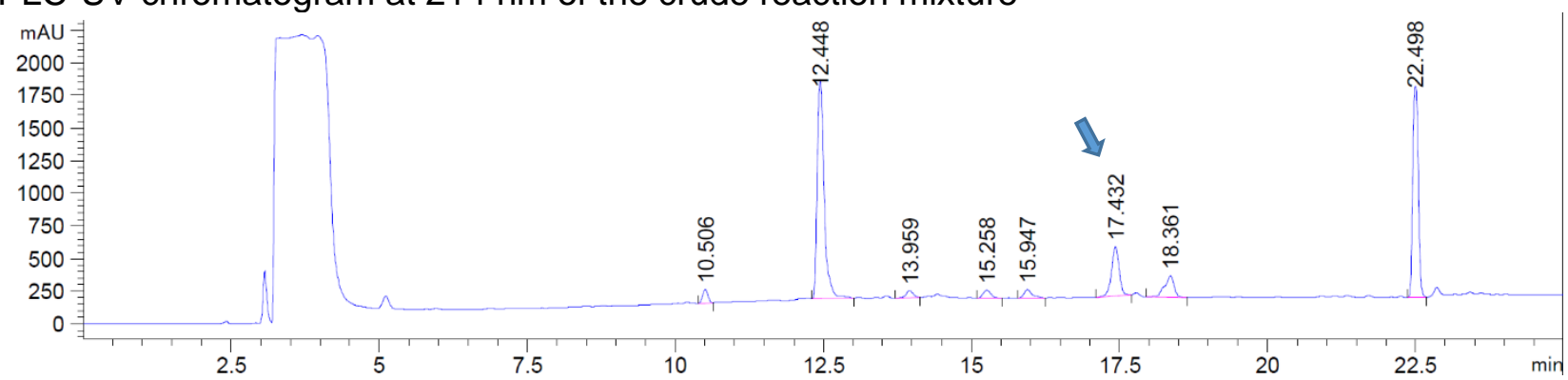

\section{Ac-Cys(Trt)-Phe-Gly-Pro-AH (11g')}<smiles>CC(=O)N[C@@H](CS[14CH3])C(=O)N[C@@H](Cc1ccccc1)C(=O)NCC(=O)N1CCCC1C#Cc1ccccc1</smiles>

Following the general procedure, Ac-Cys(Trt)-Phe-Gly-Pro-OH (10g') afforded 11g' in 94\% yield (retention time 20.083).

HRMS (nanochip-ESI/LTQ-Orbitrap) m/z: $[\mathrm{M}+\mathrm{H}]^{+}$Calcd for $\mathrm{C}_{47} \mathrm{H}_{47} \mathrm{~N}_{4} \mathrm{O}_{4} \mathrm{~S}^{+}$763.3313; Found 763.3333.

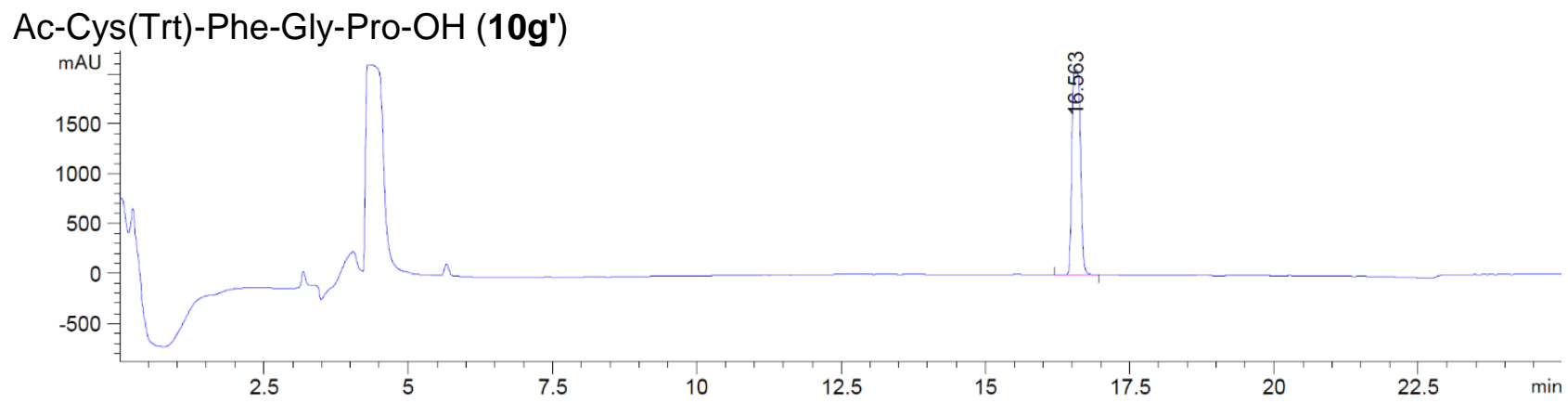

HRMS of 10g' (nanochip-ESI/LTQ-Orbitrap) m/z: [M + H] $]^{+}$Calcd for $\mathrm{C}_{40} \mathrm{H}_{43} \mathrm{~N}_{4} \mathrm{O}_{6} \mathrm{~S}^{+}$707.2898; Found 707.2909.

HPLC-UV chromatogram at $214 \mathrm{~nm}$ of the crude reaction mixture

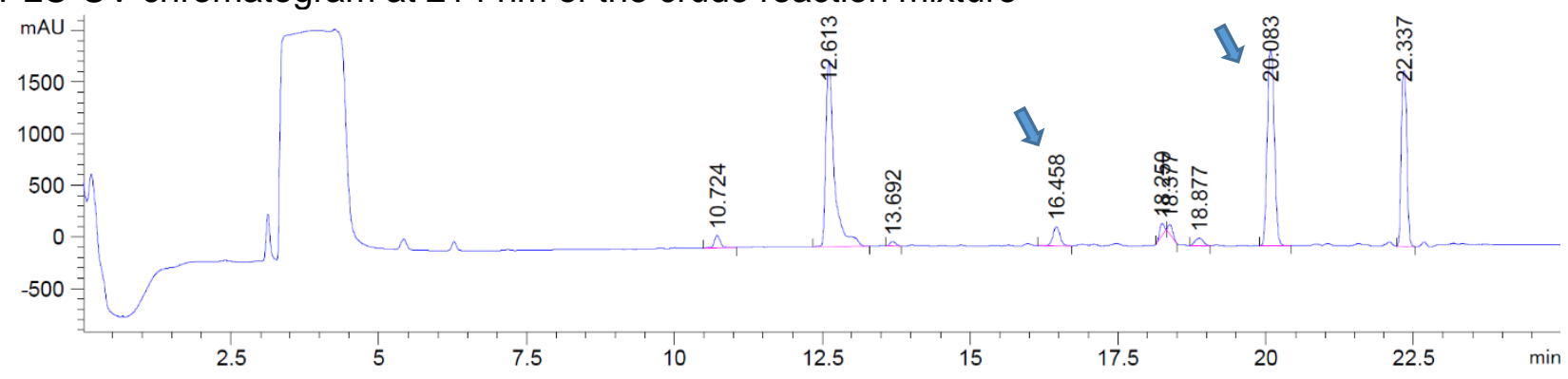

Ac-Ala-Phe-Gly-Ala-AH (11h) 
<smiles>CC(=O)N[C@@H](C)C(=O)N[C@@H](Cc1ccccc1)C(=O)NCC(=O)NC(C)C#Cc1ccccc1</smiles>

Following the general procedure, Ac-Ala-Phe-Gly-Ala-OH (10h) afforded $\mathbf{1 1 h}$ in more than 95\% yield (retention time 12.755).

HRMS (ESI/QTOF) m/z: [M + K]+ Calcd for $\mathrm{C}_{26} \mathrm{H}_{30} \mathrm{KN}_{4} \mathrm{O}_{4}{ }^{+}$501.1899; Found 501.1908.

Ac-Ala-Phe-Gly-Ala-OH (10h)

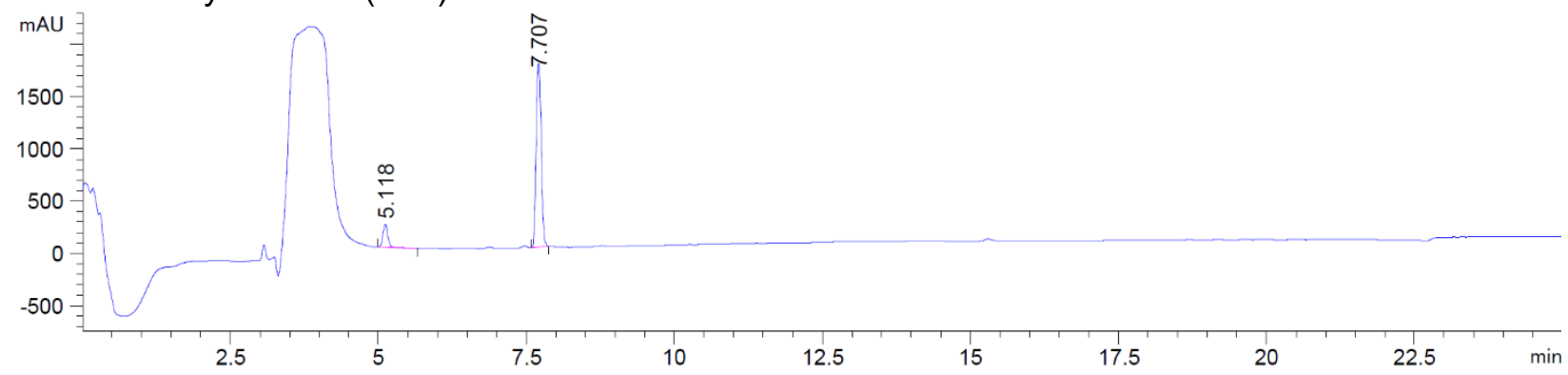

HRMS of 10h (ESI/QTOF) m/z: [M + Na] ${ }^{+}$Calcd for $\mathrm{C}_{19} \mathrm{H}_{26} \mathrm{~N}_{4} \mathrm{NaO}_{6}{ }^{+}$429.1745; Found 429.1736.

HPLC-UV chromatogram at $214 \mathrm{~nm}$ of the crude reaction mixture

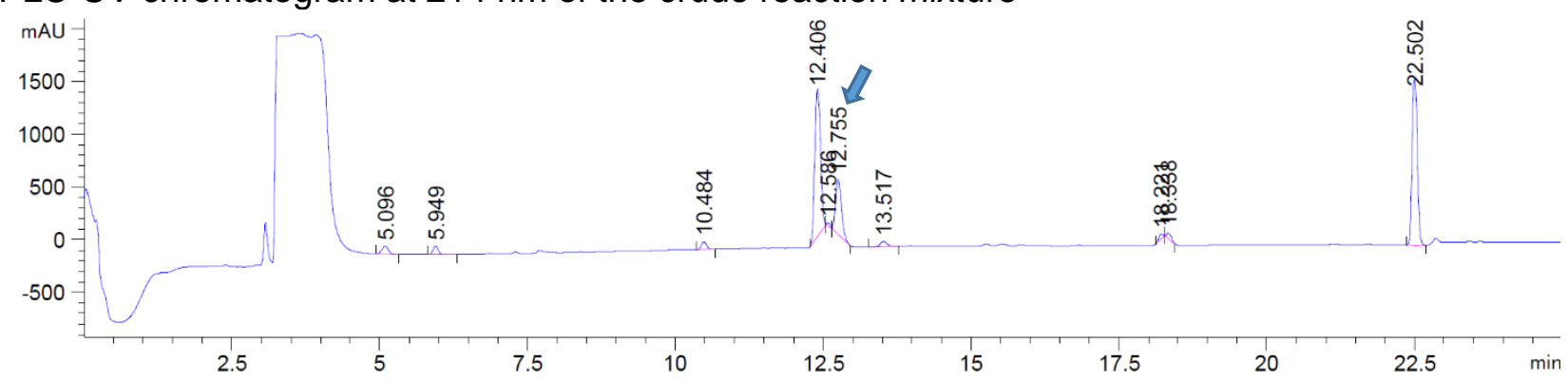

Ac-Ala-Phe-Gly-Phe-AH (11i)<smiles>CC(=O)N[C@@H](C)C(=O)N[C@@H](Cc1ccccc1)C(=O)NCC(=O)NC(C#Cc1ccccc1)Cc1ccccc1</smiles>

Following the general procedure, Ac-Ala-Phe-Gly-Phe-OH (10i) afforded 11i in more than 95\% yield (retention time 15.242).

HRMS (QTOF) m/z: [M + K] $]^{+}$Calcd for $\mathrm{C}_{32} \mathrm{H}_{34} \mathrm{KN}_{4} \mathrm{O}_{4}{ }^{+}$577.2212; Found 577.2225.

Ac-Ala-Phe-Gly-Phe-OH (10i) 


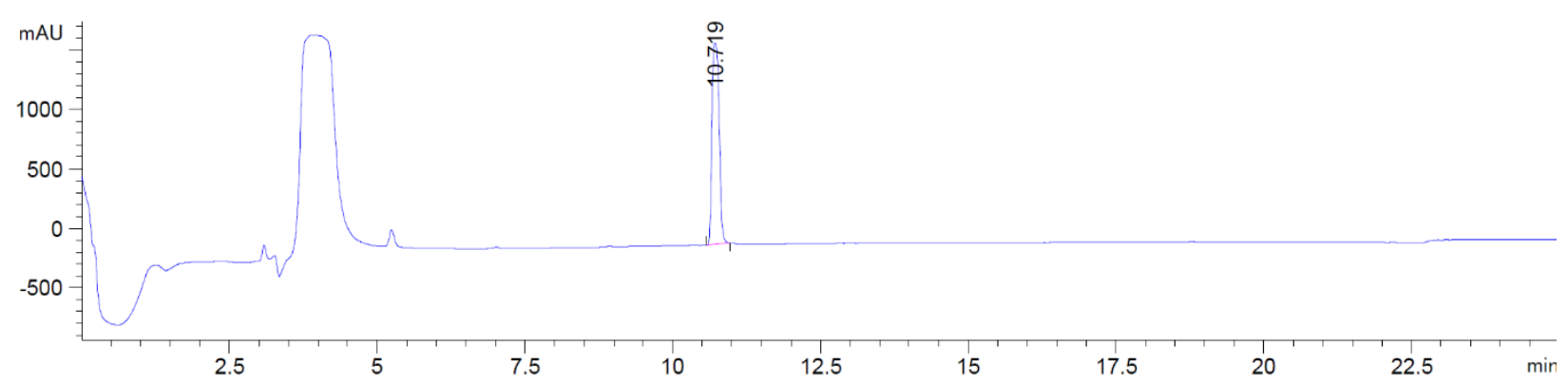

HRMS of 10i (ESI/QTOF) m/z: [M + Na] ${ }^{+}$Calcd for $\mathrm{C}_{25} \mathrm{H}_{30} \mathrm{~N}_{4} \mathrm{NaO}_{6}{ }^{+}$505.2058; Found 505.2069.

HPLC-UV chromatogram at $214 \mathrm{~nm}$ of the crude reaction mixture

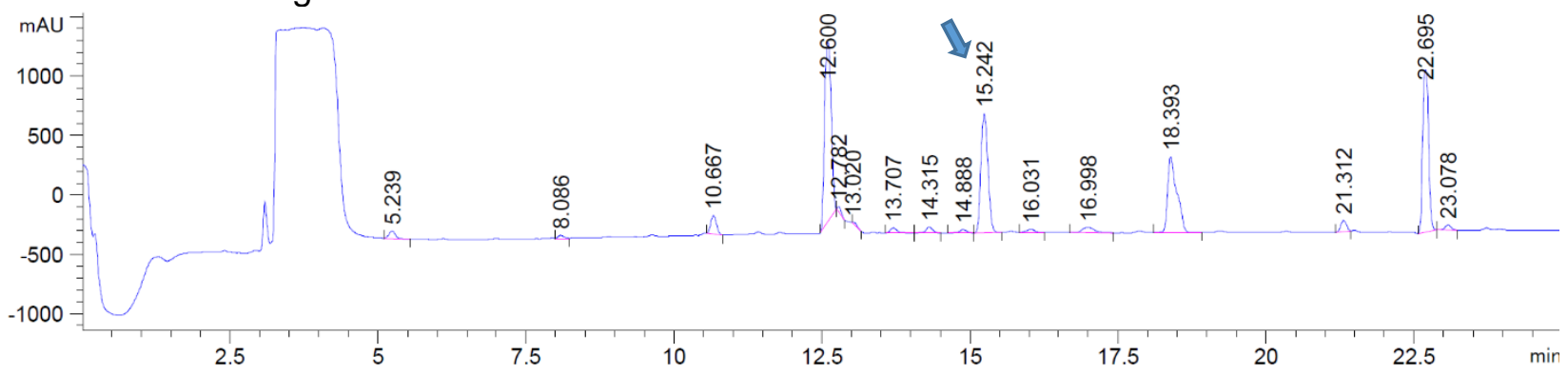

Ac-Ala-Phe-Gly-Arg-AH (11j)<smiles>CC(=O)N[C@@H](C)C(=O)N[C@@H](Cc1ccccc1)C(=O)NCC(=O)NC(C#CCc1ccccc1)CCCNC(=N)N</smiles>

Following the general procedure, Ac-Ala-Phe-Gly-Arg-OH (10j) afforded $\mathbf{1 1} \mathbf{j}$ in more than $95 \%$ yield (retention time 9.735).

HRMS (ESI/QTOF) m/z: [M + H] Calcd for $\mathrm{C}_{29} \mathrm{H}_{38} \mathrm{~N}_{7} \mathrm{O}_{4}+$ 548.2980; Found 548.2988.

Ac-Ala-Phe-Gly-Arg-OH (10j)

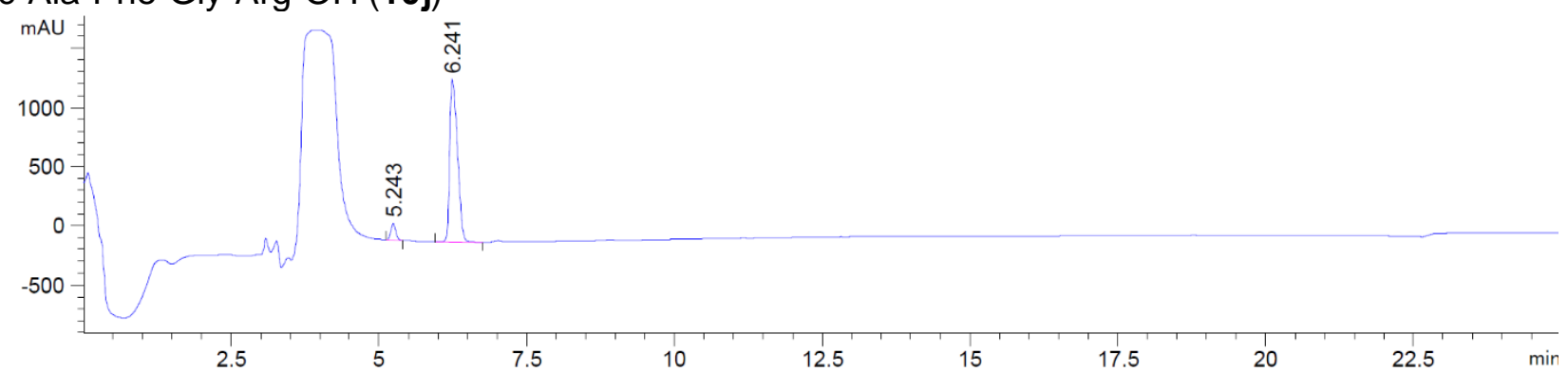

HRMS of 10j (ESI/QTOF) m/z: [M + H] $]^{+}$Calcd for $\mathrm{C}_{22} \mathrm{H}_{34} \mathrm{~N}_{7} \mathrm{O}_{6}+492.2565$; Found 492.2576.

HPLC-UV chromatogram at $214 \mathrm{~nm}$ of the crude reaction mixture 


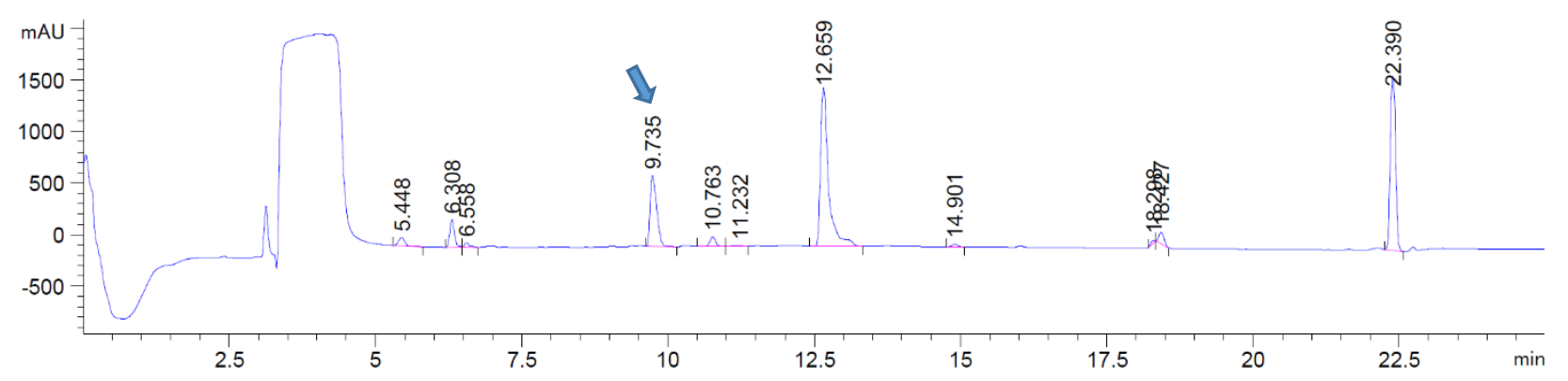

Ac-Ala-Phe-Gly-Ser-AH (11k)<smiles>CC(=O)N[C@@H](C)C(=O)NC(Cc1ccccc1)C(=O)NCC(=O)NC(C#Cc1ccccc1)CO</smiles>

Following the general procedure, Ac-Ala-Phe-Gly-Ser-OH (10k) afforded 11k in more than 95\% yield (retention time 11.436).

HRMS (ESI/QTOF) m/z: [M + Na] ${ }^{+}$Calcd for $\mathrm{C}_{26} \mathrm{H}_{30} \mathrm{~N}_{4} \mathrm{NaO}_{5}{ }^{+}$501.2108; Found 501.2107.

Ac-Ala-Phe-Gly-Ser-OH (10k)

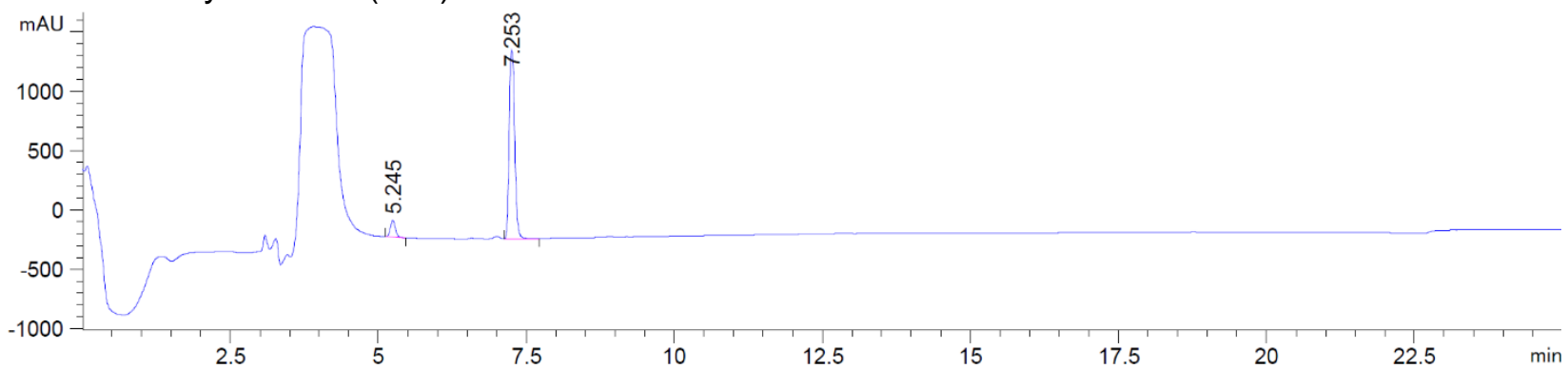

HRMS of 10k (ESI/QTOF) m/z: [M + Na] ${ }^{+}$Calcd for $\mathrm{C}_{19} \mathrm{H}_{26} \mathrm{~N}_{4} \mathrm{NaO}_{7}{ }^{+} 445.1694$; Found 445.1688

HPLC-UV chromatogram at $214 \mathrm{~nm}$ of the crude reaction mixture

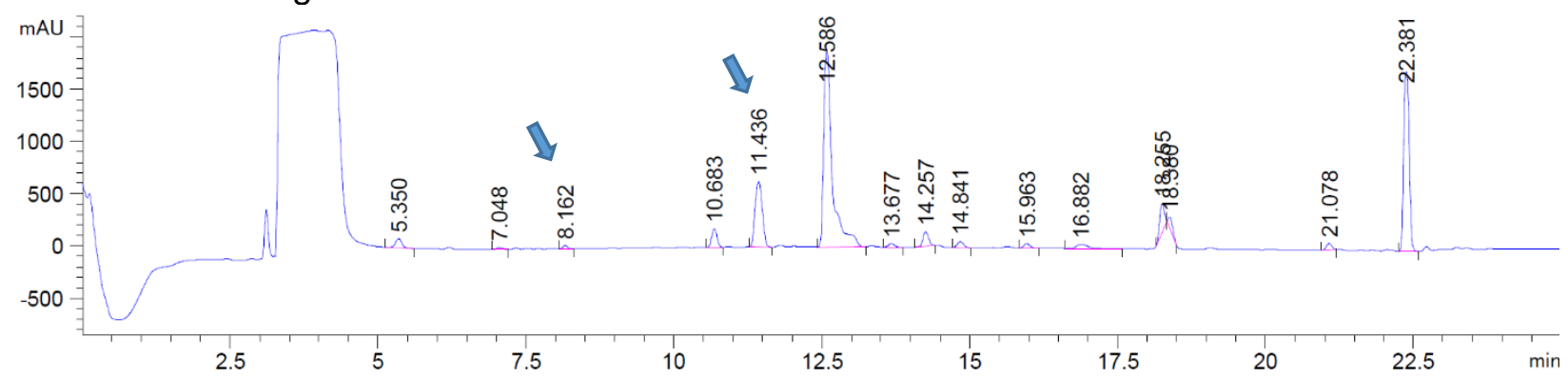

Ac-Ala-Phe-Gly-Met-AH (11) 
<smiles>CSCCC(C#CPc1ccccc1)NC(=O)CNC(=O)C(Cc1ccccc1)NC(=O)[C@H](C)NC(C)=O</smiles>

Following the general procedure, Ac-Ala-Phe-Gly-Met-OH (10I) afforded $\mathbf{1 1 I}$ in more than 95\% yield (retention time 12.124).

HRMS (ESI/QTOF) m/z: [M + H] $]^{+}$Calcd for $\mathrm{C}_{28} \mathrm{H}_{35} \mathrm{~N}_{4} \mathrm{O}_{4} \mathrm{~S}^{+}$523.2374; Found 523.2375

Ac-Ala-Phe-Gly-Met-OH (10l)

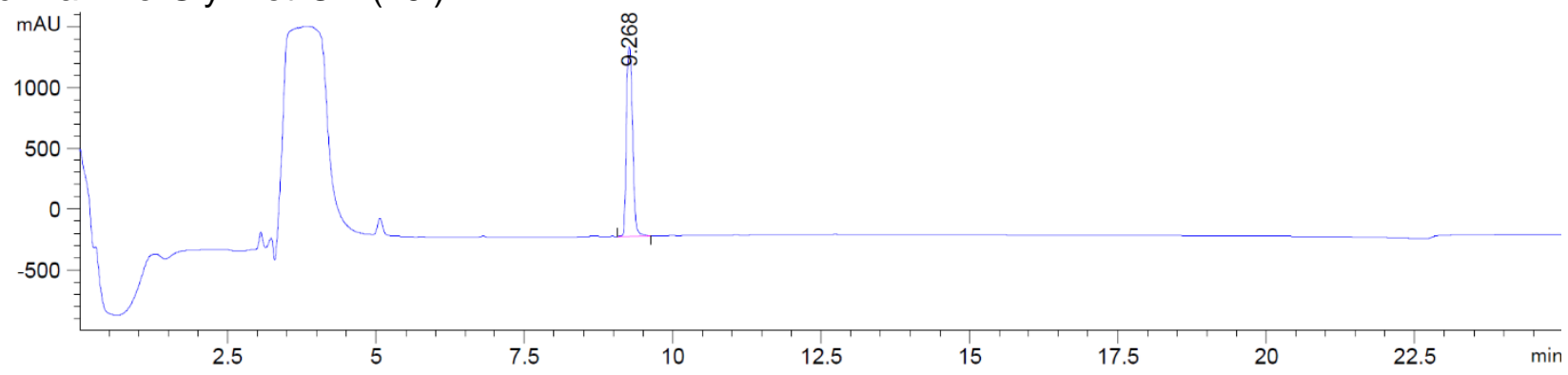

HRMS of 10l (ESI/QTOF) m/z: [M + Na]+ Calcd for C21H30N4NaO6S+ 489.1778; Found 489.1774.

HPLC-UV chromatogram at $214 \mathrm{~nm}$ of the crude reaction mixture

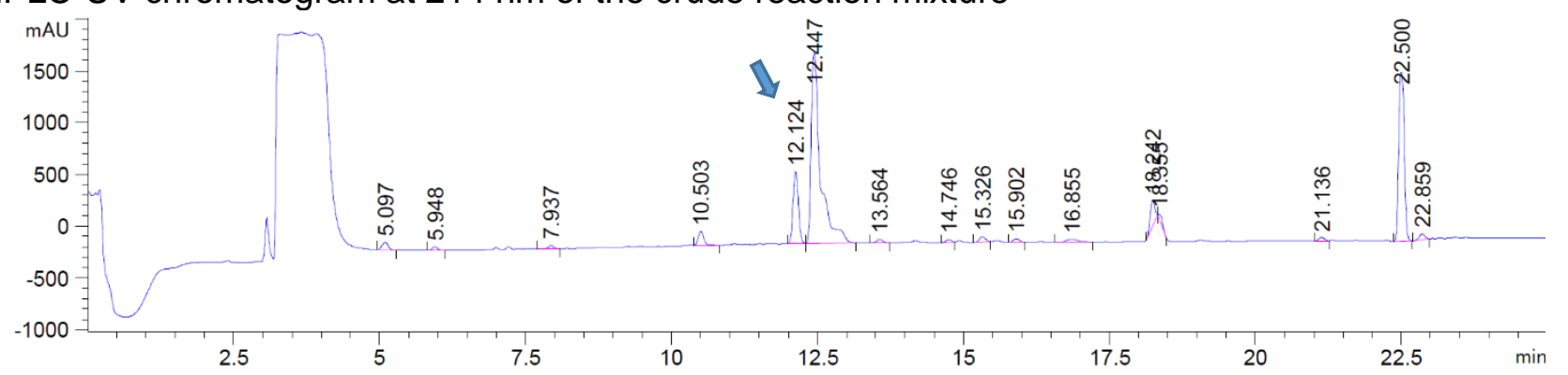

\section{Ac-Ala-Phe-Gly-Asn-AH (11m)}<smiles>CC(=O)N[C@@H](C)C(=O)N[C@@H](Cc1ccccc1)C(=O)NCC(=O)NC(C#CPc1ccccc1)CC(N)=O</smiles>

Following the general procedure, Ac-Ala-Phe-Gly-Asn-OH (10m) afforded $\mathbf{1 1 h}$ in more than $95 \%$ yield (retention time 10.724).

HRMS (ESI/QTOF) m/z: [M + Na] ${ }^{+}$Calcd for $\mathrm{C}_{27} \mathrm{H}_{31} \mathrm{~N}_{5} \mathrm{NaO}_{5}{ }^{+}$528.2217; Found 528.2223.

Ac-Ala-Phe-Gly-Asn-OH (10m) 


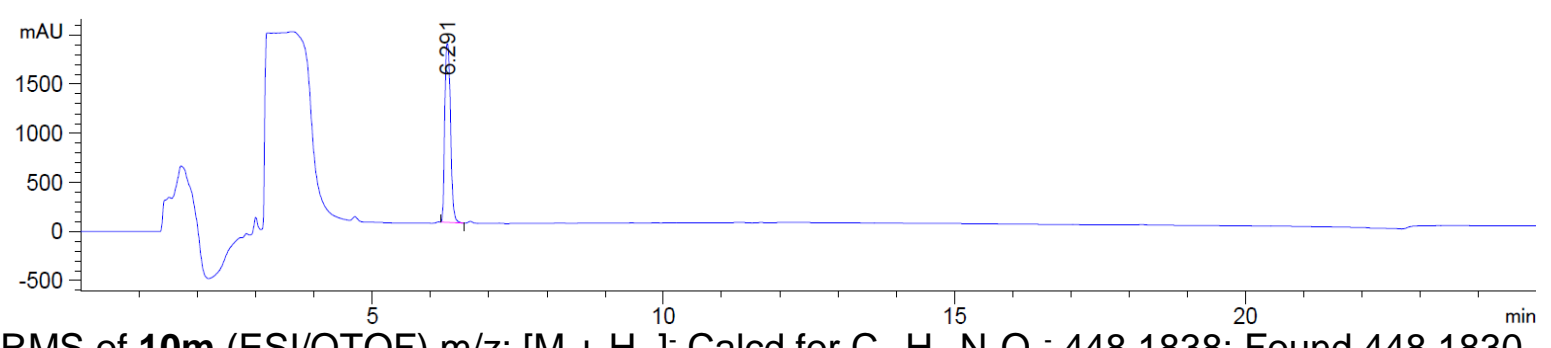

HRMS of $10 \mathrm{~m}$ (ESI/QTOF) m/z: [M + $\left.\mathrm{H}_{-1}^{10}\right]^{-}$Calcd for $\mathrm{C}_{20} \mathrm{H}_{26}^{15} \mathrm{~N}_{5} \mathrm{O}_{7}^{-} 448.1838^{20}$ Found 448.1830.

HPLC-UV chromatogram at $214 \mathrm{~nm}$ of the crude reaction mixture

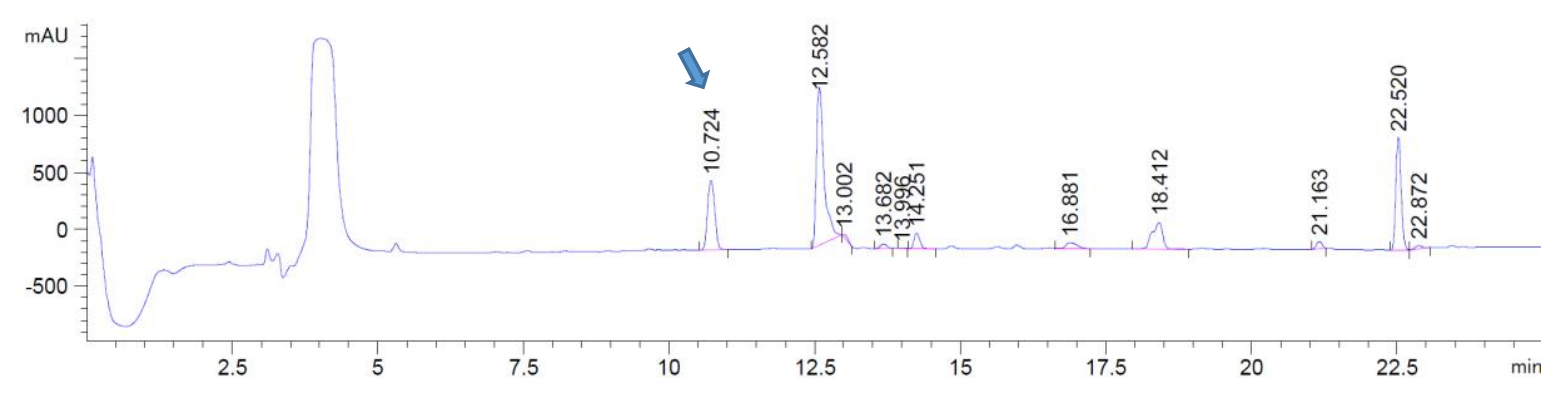

\section{Ac-Ala-Phe-Gly-Gly-AH (11n)}<smiles>CC(=O)N[C@@H](C)C(=O)N[C@@H](Cc1ccccc1)C(=O)NCC(=O)NCC#Cc1ccccc1</smiles>

Following the general procedure, Ac-Ala-Phe-Gly-Gly-OH (10n) afforded 11n in more than 95\% yield (retention time 11.995).

HRMS (ESI/QTOF) m/z: [M + K] $]^{+}$Calcd for $\mathrm{C}_{25} \mathrm{H}_{28} \mathrm{KN}_{4} \mathrm{O}_{4}{ }^{+}$487.1742; Found 487.1757.

Ac-Ala-Phe-Gly-Gly-OH (10n)

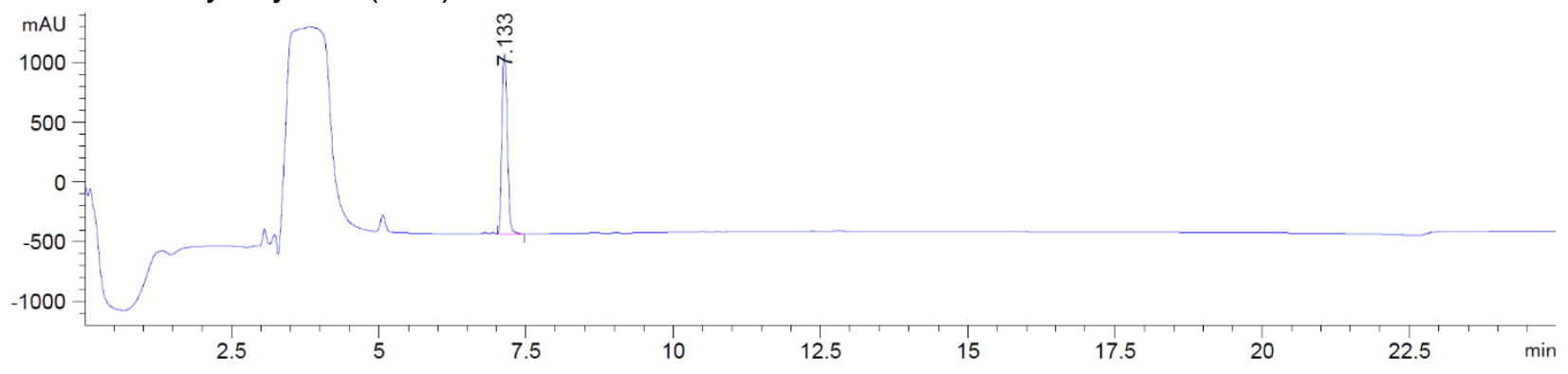

HRMS of 10n (ESI/QTOF) m/z: [M + Na] ${ }^{+}$Calcd for $\mathrm{C}_{18} \mathrm{H}_{24} \mathrm{~N}_{4} \mathrm{NaO}_{6}{ }^{+} 415.1588$; Found 415.1585.

HPLC-UV chromatogram at $214 \mathrm{~nm}$ of the crude reaction mixture 


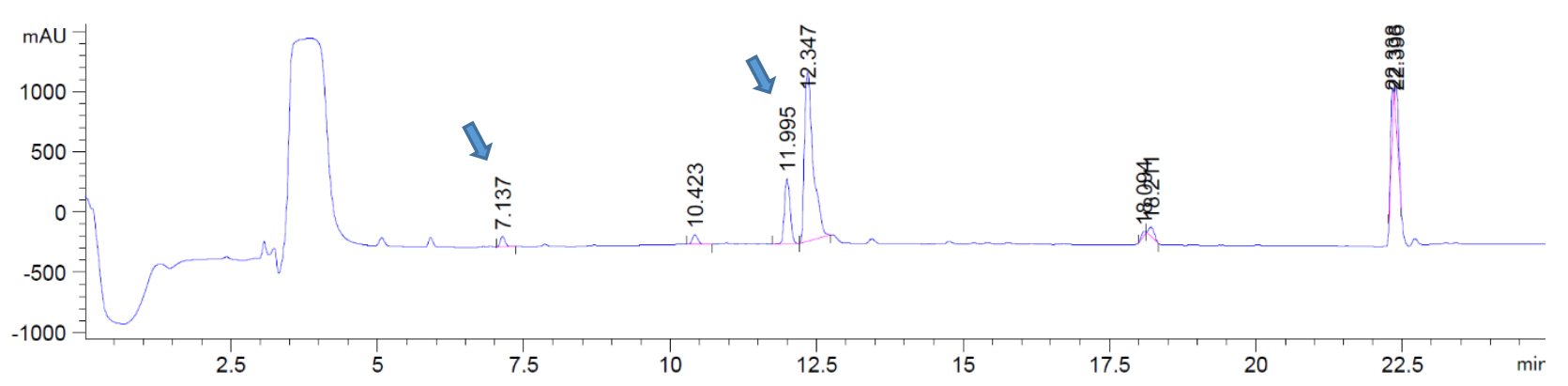

\section{Ac-Ala-Phe-Gly-His-AH (110)}<smiles>CC(=O)N[C@@H](C)C(=O)N[C@@H](Cc1ccccc1)C(=O)NCC(=O)NC(C#Cc1ccccc1)Cc1cnc[nH]1</smiles>

Following the general procedure, Ac-Ala-Phe-Gly-His-OH (100) afforded 110 in an average of $76 \%$ yield (retention time 9.386).

HRMS (ESI/QTOF) m/z: [M + H] Calcd for $\mathrm{C}_{29} \mathrm{H}_{33} \mathrm{~N}_{6} \mathrm{O}_{4}{ }^{+}$529.2558; Found 529.2566.

Ac-Ala-Phe-Gly-His-OH (100)

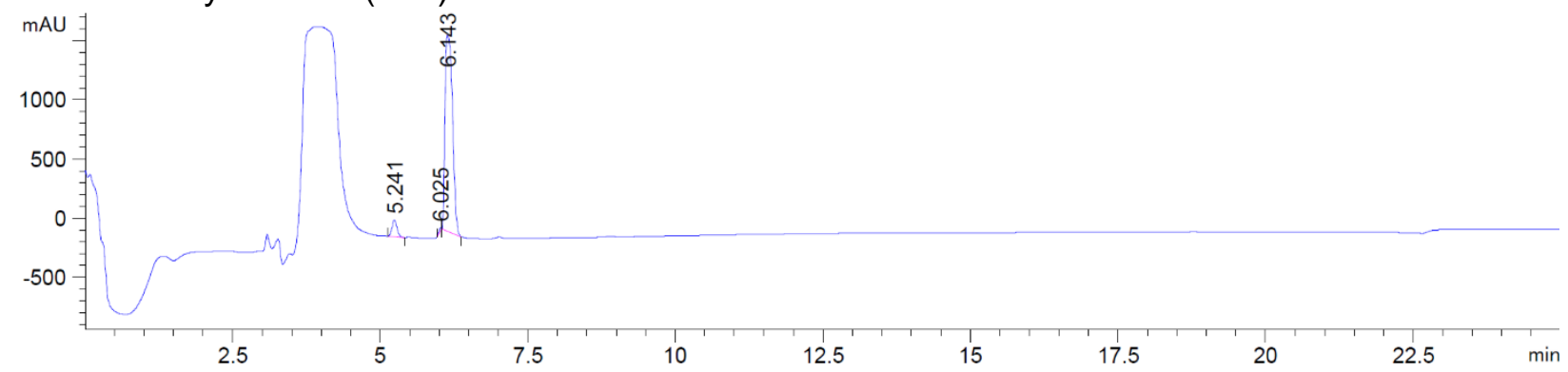

HRMS of 100 (ESI/QTOF) m/z: [M + H] $]^{+}$Calcd for $\mathrm{C}_{22} \mathrm{H}_{29} \mathrm{~N}_{6} \mathrm{O}_{6}{ }^{+}$473.2143; Found 473.2148.

HPLC-UV chromatogram at $214 \mathrm{~nm}$ of the crude reaction mixture

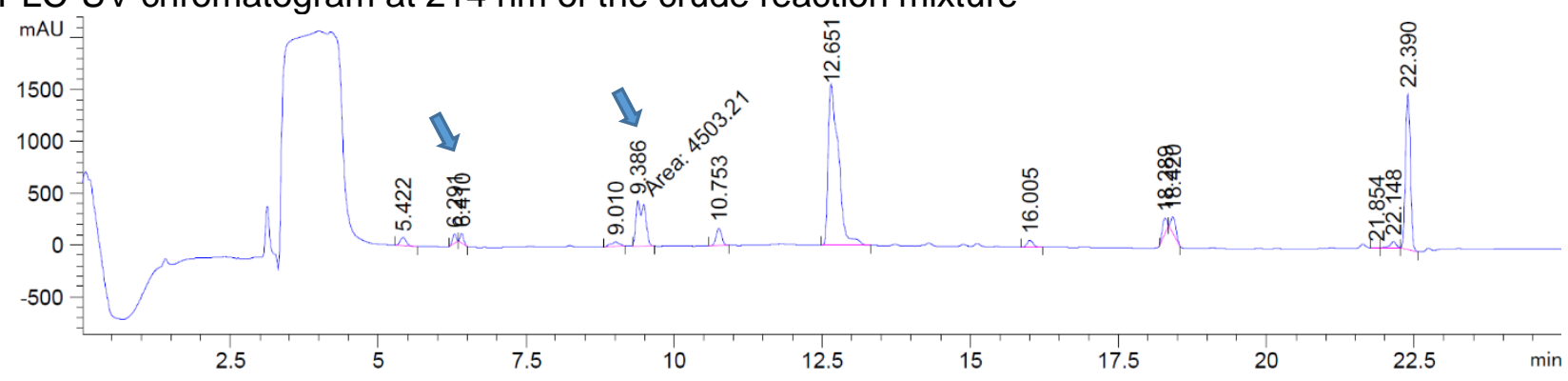

Ac-Ala-Phe-Gly-Lys-AH (11p)<smiles>CC(=O)N[C@@H](C)C(=O)N[C@@H](Cc1ccccc1)C(=O)NCC(=O)NC(C#Cc1ccccc1)CCCCN</smiles> 
Following the general procedure, Ac-Ala-Phe-Gly-Lys-OH (10p) afforded 11p in an average of 17\% yield (retention time 9.432).

HRMS (ESI/QTOF) m/z: [M + H] $]^{+}$Calcd for $\mathrm{C}_{29} \mathrm{H}_{38} \mathrm{~N}_{5} \mathrm{O}_{4}{ }^{+}$520.2918; Found 520.2922.

Ac-Ala-Phe-Gly-Lys-OH (10p)

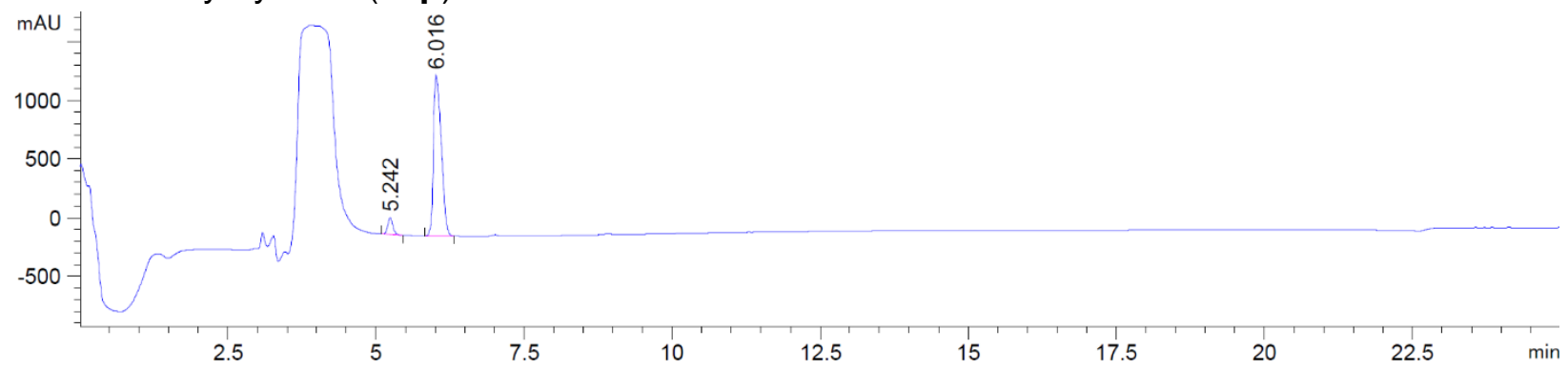

HRMS of 10p (ESI/QTOF) m/z: [M + H] $]^{+}$Calcd for $\mathrm{C}_{22} \mathrm{H}_{34} \mathrm{~N}_{5} \mathrm{O}_{6}{ }^{+} 464.2504$; Found 464.2514.

HPLC-UV chromatogram at $214 \mathrm{~nm}$ of the crude reaction mixture

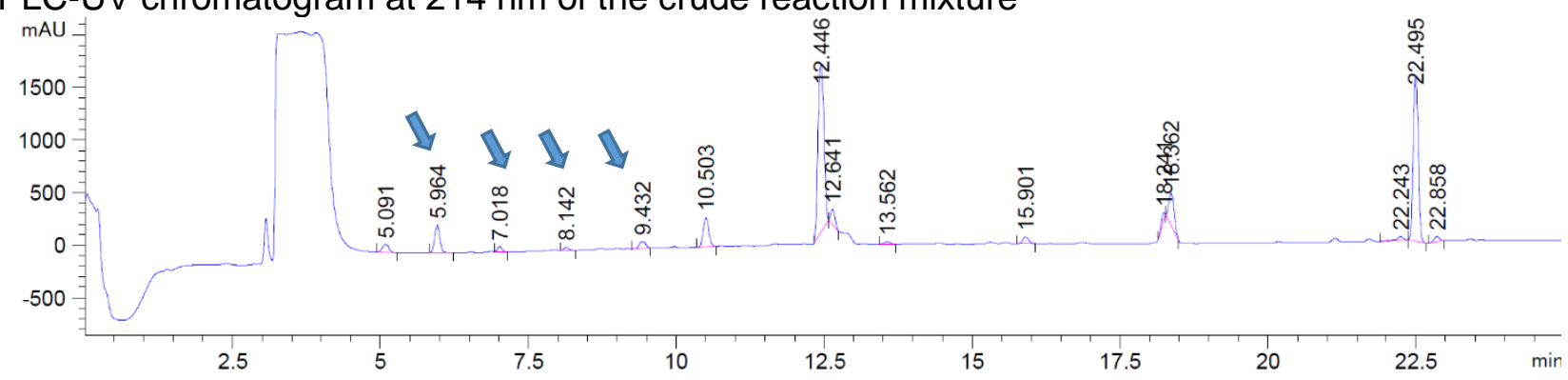

\section{Ac-Ala-Phe-Gly-Lys(Boc)-AH (11p')}

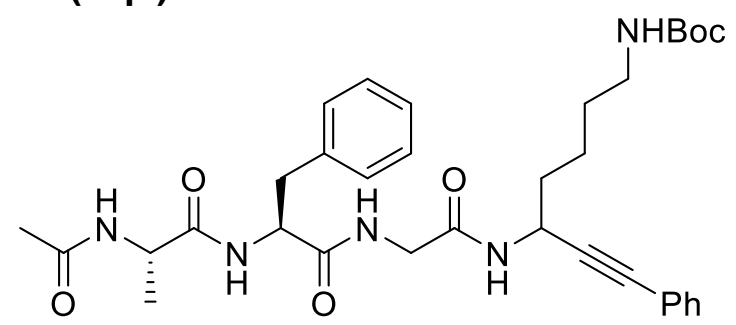

Following the general procedure, Ac-Ala-Phe-Gly-Lys(Boc)-OH (10p') afforded 11p' in more than $95 \%$ yield (retention time 15.359).

HRMS (nanochip-ESI/LTQ-Orbitrap) $\mathrm{m} / \mathrm{z}:[\mathrm{M}+\mathrm{H}]^{+}$Calcd for $\mathrm{C}_{34} \mathrm{H}_{46} \mathrm{~N}_{5} \mathrm{O}_{6}+620.3443$; Found 620.3455.

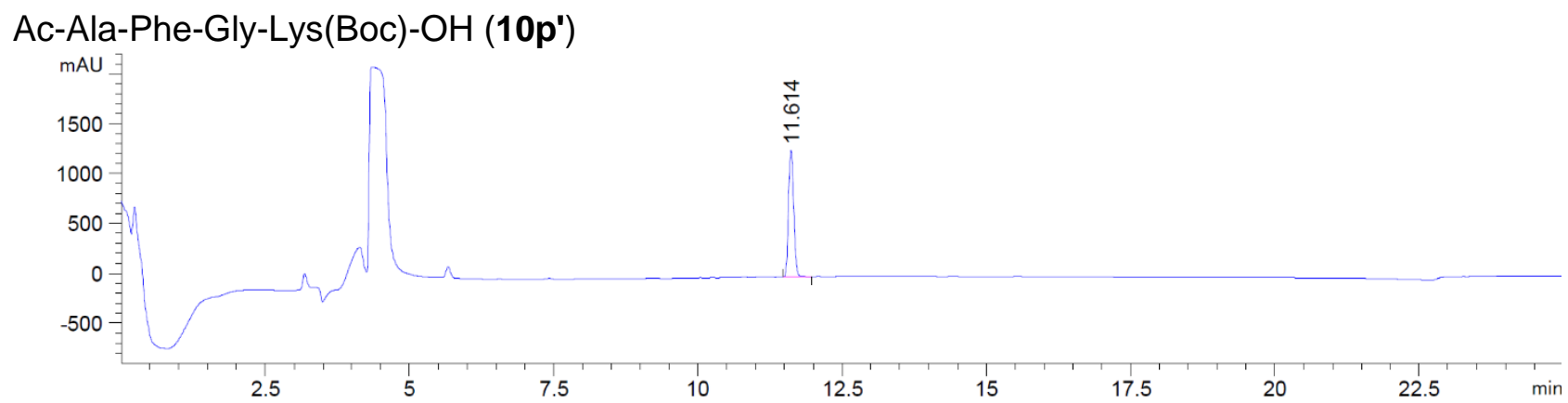


HRMS of 10p' (ESI/QTOF) m/z: [M + Na] ${ }^{+}$Calcd for $\mathrm{C}_{27} \mathrm{H}_{41} \mathrm{~N}_{5} \mathrm{NaO}_{8}{ }^{+}$586.2847; Found 586.2860.

HPLC-UV chromatogram at $214 \mathrm{~nm}$ of the crude reaction mixture

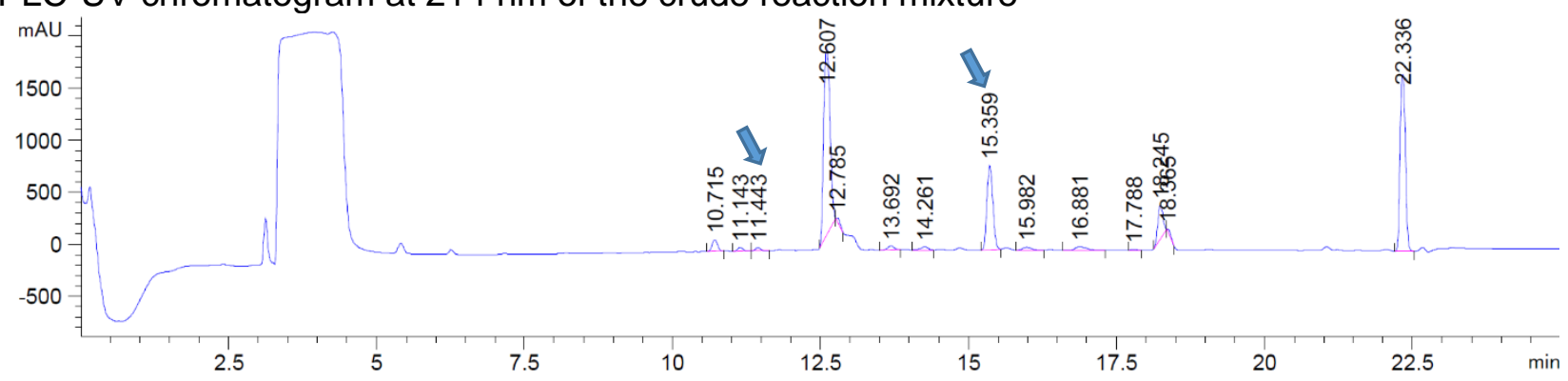

\section{Ac-Ala-Phe-Gly-Tyr-AH (11q)}<smiles>CC(=O)N[C@@H](C)C(=O)N[C@@H](Cc1ccccc1)C(=O)NCC(=O)NC(C#Cc1ccccc1)Cc1ccc(O)cc1</smiles>

Following the general procedure and using Ir (4) as catalyst, Ac-Ala-Phe-Gly-Tyr-OH (10q) afforded $11 q$ in an average of $29 \%$ yield (retention time 13.243).

HRMS (ESI/QTOF) m/z: [M + K] $]^{+}$Calcd for $\mathrm{C}_{32} \mathrm{H}_{34} \mathrm{KN}_{4} \mathrm{O}_{5}{ }^{+}$593.2161; Found 593.2172.

Ac-Ala-Phe-Gly-Tyr-OH (10q)

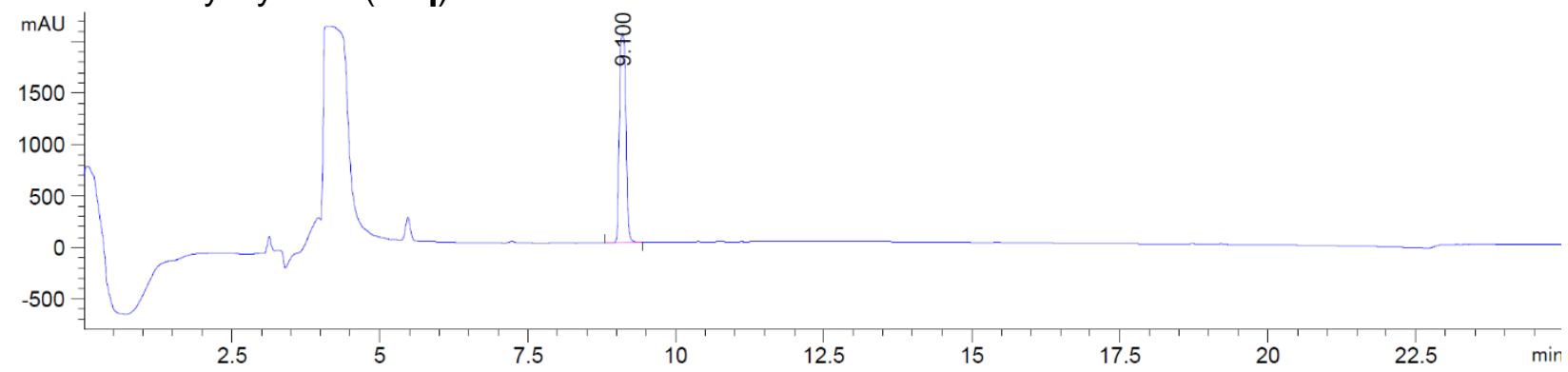

HRMS of $10 q$ (ESI/QTOF) m/z: [M + H] ${ }^{+}$Calcd for $\mathrm{C}_{25} \mathrm{H}_{31} \mathrm{~N}_{4} \mathrm{O}_{7}+499.2187$; Found 499.2187.

HPLC-UV chromatogram at $214 \mathrm{~nm}$ of the crude reaction mixture

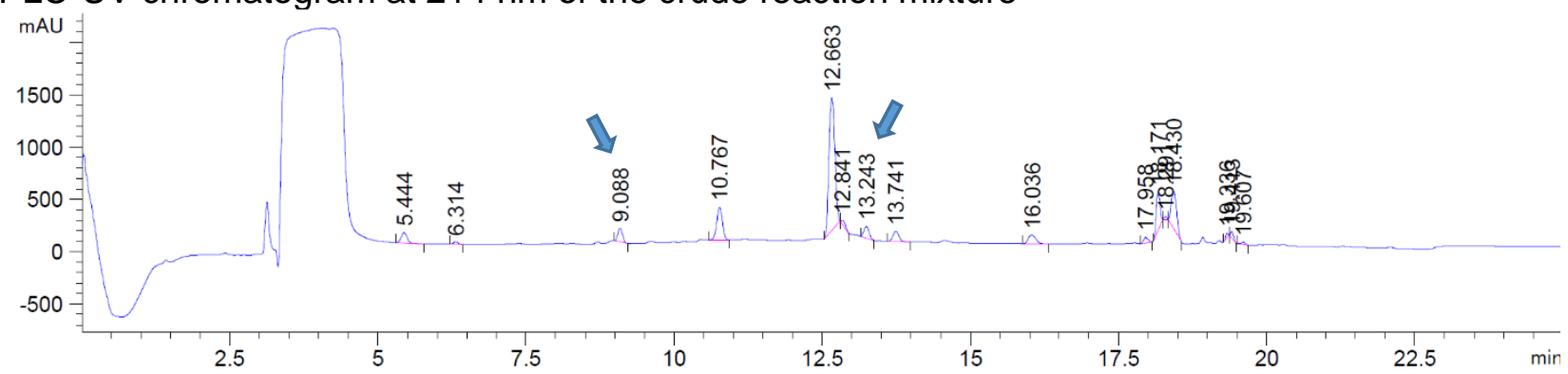


<smiles>CC(=O)N[C@@H](C)C(=O)N[C@@H](Cc1ccccc1)C(=O)NCC(=O)NC(C#Cc1ccccc1)CCC(=O)O</smiles>

Following the general procedure, Ac-Ala-Phe-Gly-Glu-OH (10r) afforded 11r in more than 95\% yield (retention time 11.730). The product with double alkynylation was detected at a retention time of 16.9 as less than $5 \%$ as the single alkynyation at the C-terminal position.

HRMS (ESI/QTOF) m/z: [M + K] $]^{+}$Calcd for $\mathrm{C}_{28} \mathrm{H}_{32} \mathrm{KN}_{4} \mathrm{O}_{6}{ }^{+}$559.1953; Found 559.1962.

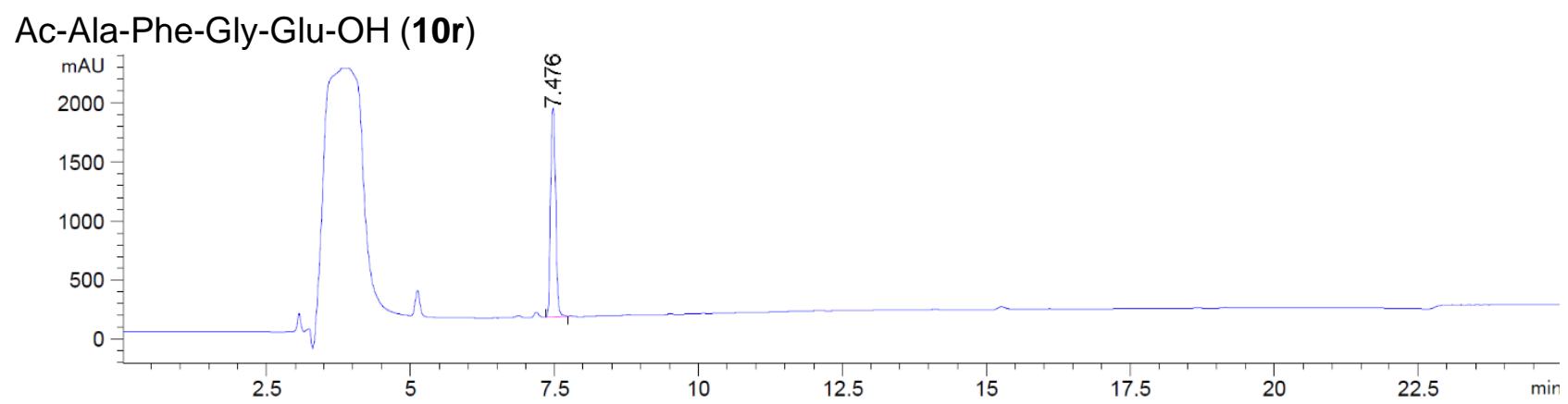

HRMS of $10 \mathrm{r}$ (ESI/QTOF) m/z: $[\mathrm{M}+\mathrm{Na}]^{+}$Calcd for $\mathrm{C}_{21} \mathrm{H}_{28} \mathrm{~N}_{4} \mathrm{NaO}_{8}{ }^{+}$487.1799; Found 487.1807.

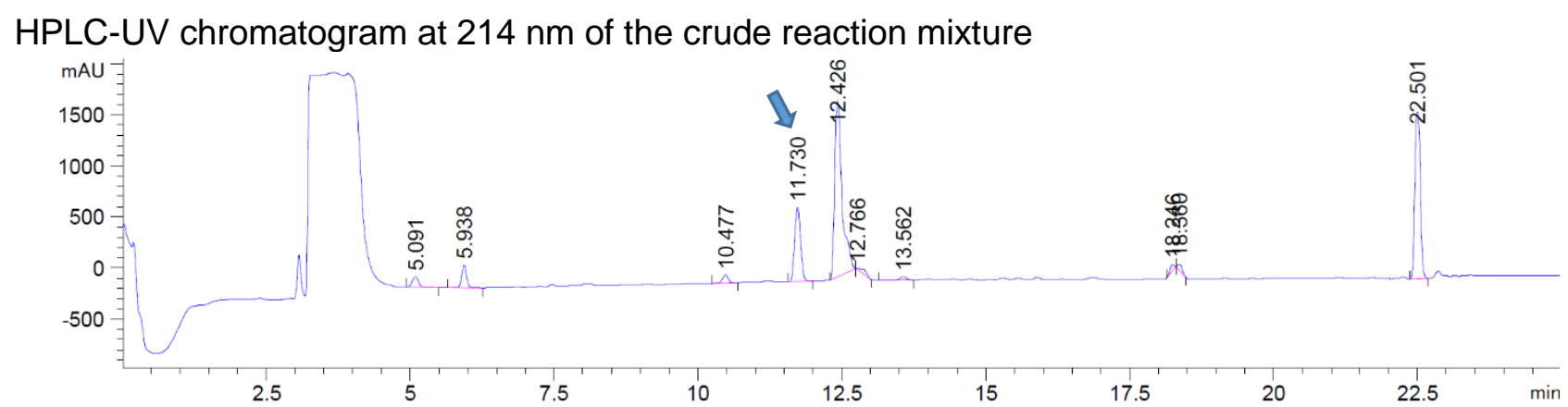

The 3 independent trials were gathered and purified by preparative HPLC using a gradient of 100\% A to $100 \%$ B in 20 minutes (see general methods). The desired peptide was obtained as a mixture at $40 \%$ pure together with iodobenzoic acid. The site of decarboxylative alkynylation was assigned by NMR. The signal in $\alpha$ position of the alkyne was attributed with the ${ }^{1} \mathrm{H}$ and the HMBC NMR. As an integration of 1 was measured, selective alkynylation at the C-terminal position was achieved. The signals are reported bellow, with signals of iodobenzoic acid in italics in brackets.

${ }^{1} \mathrm{H}$ NMR (800 MHz, DMF- $d_{7}, 1: 1$ mixture of diastereoisomers, signals only partially resolved, only relevant protons were attributed) $\delta 8.29-8.10(\mathrm{~m}, 4 \mathrm{H}, \mathrm{ArH}),(8.07(\mathrm{dd}, \mathrm{J}=7.9,1.1 \mathrm{~Hz}, 2 \mathrm{H}), 8.03(\mathrm{~s}$, 4H), $7.85(d d, J=7.7,1.7 \mathrm{~Hz}, 2 \mathrm{H}), 7.55(t d, J=7.5,1.2 \mathrm{~Hz}, 2 \mathrm{H})) ,7.46(\mathrm{tdd}, J=4.8,3.1,1.9 \mathrm{~Hz}, 2 \mathrm{H}$, $\operatorname{ArH}), 7.42-7.37(\mathrm{~m}, 3 \mathrm{H}, \mathrm{ArH}),(7.32-7.27(\mathrm{~m}, 6 \mathrm{H})) 7.24-,7.18(\mathrm{~m}, 1 \mathrm{H}, \operatorname{Ar} H), 5.08-4.97(\mathrm{~m}, 1 \mathrm{H}$, $\mathrm{NCHC} \equiv \mathrm{C}$ ), 4.56 (ddd, $J=9.5,7.5,4.8 \mathrm{~Hz}, 0.5 \mathrm{H}, \mathrm{NC}(\mathrm{O}) \mathrm{CH}_{2} \mathrm{NH}$ ), 4.52 (ddd, $J=9.6,7.4,4.8 \mathrm{~Hz}, 0.5 \mathrm{H}$, $\left.\mathrm{NC}(\mathrm{O}) \mathrm{CH}_{2} \mathrm{NH}\right), 4.32-4.24\left(\mathrm{~m}, 1 \mathrm{H}, \mathrm{NC}(\mathrm{O}) \mathrm{CHCH}_{3} \mathrm{NH}\right), 3.97(\mathrm{dd}, J=6.3,1.6 \mathrm{~Hz}, 1 \mathrm{H}), 3.95(\mathrm{dd}, J=$ 6.3, $1.5 \mathrm{~Hz}, 1 \mathrm{H}), 3.82(\mathrm{dd}, J=16.7,5.6 \mathrm{~Hz}, 0.5 \mathrm{H}), 3.79(\mathrm{dd}, J=16.7,5.6 \mathrm{~Hz}, 0.5 \mathrm{H}$ ), 3.25 (ddd, $J=$ 14.0, 6.8, 4.8 Hz, 1H), $3.06-2.97(\mathrm{~m}, 1 \mathrm{H}), 2.59-2.48\left(\mathrm{~m}, 2 \mathrm{H}, \mathrm{CHCH}_{2} \mathrm{CH}_{3}\right), 2.12-2.02(\mathrm{~m}, 2 \mathrm{H}$, $\left.\mathrm{NHCHCH}_{2}\right), 1.90(\mathrm{~d}, J=3.4 \mathrm{~Hz}, 3 \mathrm{H}, A c-\mathrm{NH}), 1.22\left(\mathrm{t}, J=6.8 \mathrm{~Hz}, 3 \mathrm{H}, \mathrm{NC}(\mathrm{O}) \mathrm{CHCH}_{3} \mathrm{NH}\right)$.

${ }^{13} \mathrm{C}$ NMR $\left(201 \mathrm{MHz}, \mathrm{DMF}-d_{7}\right) \delta 174.3,174.2,173.6,173.5,171.8,170.6,170.5,168.7,(168.3$, 141.4), 138.6, 138.6, (137.4, 132.8), 132.6, 131.9, 131.9, (130.8), 129.6, 129.3, 128.9, 128.9, 128.9, 
128.6, (128.5), 126.6, 123.0, (93.9), 89.5, 89.5, 82.6, 82.6, 55.4, 55.3, 49.7, 42.8, 40.9, 40.9, 37.2, $31.2,22.4,17.3,17.3$.

\section{Ac-Ala-Phe-Gly-Asp-AH (11s)}<smiles>CC(=O)N[C@@H](C)C(=O)N[C@@H](Cc1ccccc1)C(=O)NCC(=O)NC(C#Cc1ccccc1)CC(=O)O</smiles>

Following the general procedure, Ac-Ala-Phe-Gly-Asp-OH (10s) afforded 11s in an average of 37\% yield (retention time 11.684). The product with double alkynylation was not detected in the HPLC chromatogram.

HRMS (nanochip-ESI/LTQ-Orbitrap) m/z: [M] - Calcd for $\mathrm{C}_{27} \mathrm{H}_{29} \mathrm{~N}_{4} \mathrm{O}_{6}{ }^{-}$505.2093; Found 505.2077.

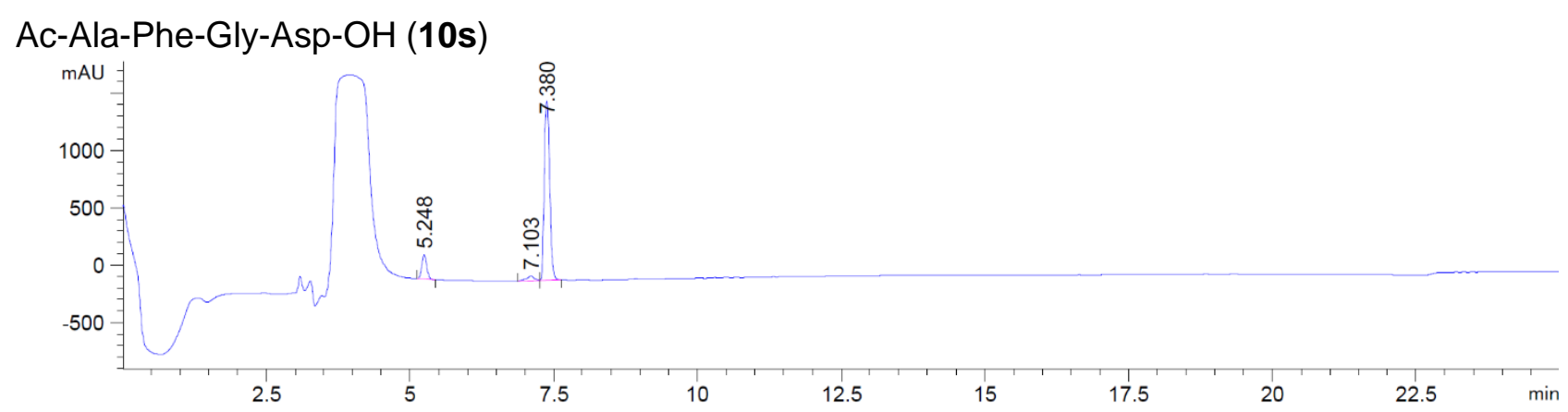

HRMS of 10s (ESI/QTOF) m/z: [M + Na] $]^{+}$Calcd for $\mathrm{C}_{20} \mathrm{H}_{26} \mathrm{~N}_{4} \mathrm{NaO}_{8}{ }^{+}$473.1643; Found 473.1646.

HPLC-UV chromatogram at $214 \mathrm{~nm}$ of the crude reaction mixture

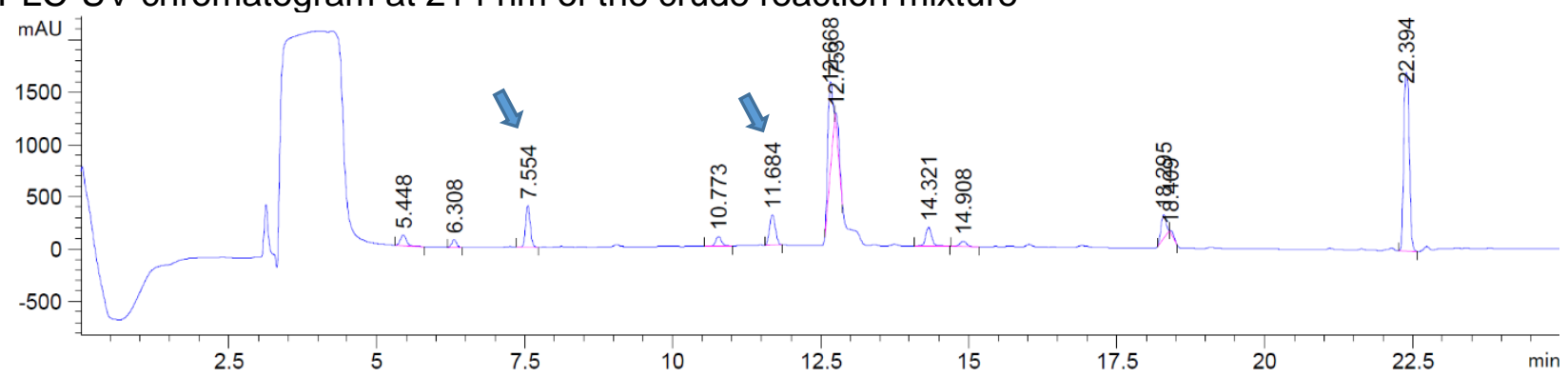

The 3 independent trials were gathered and purified by preparative HPLC using a gradient of 100\% A to $100 \%$ B in 20 minutes (see general methods). The desired peptide was obtained as a mixture at $75 \%$ pure together with iodobenzoic acid. The site of decarboxylative alkynylation was assigned by NMR by analogy to Ac-Ala-Phe-Gly-Glu-AH (11r) as not enough material was obtained to record an interpretable ${ }^{13} \mathrm{C}$ or HMBC NMR. The signal in $\alpha$ position of the alkyne was attributed with the ${ }^{1} \mathrm{H}$ NMR. As an integration of 1 was measured, selective alkynylation at the C-terminal position was achieved. The signals are reported bellow, with signals of iodobenzoic acid in italics in brackets.

${ }^{1} \mathrm{H}$ NMR $\left(800 \mathrm{MHz}, \mathrm{DMF}-d_{7}, 1: 1\right.$ mixture of diastereoisomers, signals only partially resolved, only relevant protons were attributed) $\delta 8.32-8.18(\mathrm{~m}, 2 \mathrm{H}, \mathrm{ArH}),(7.81(\mathrm{~d}, \mathrm{~J}=7.5 \mathrm{~Hz}, 0.3 \mathrm{H}), 7.54-7.49$ $(m, 0.3 H)), 7.46-7.38(\mathrm{~m}, 5 \mathrm{H}, \mathrm{ArH}), 7.33-7.26(\mathrm{~m}, 2 \mathrm{H}, \mathrm{ArH}($ and $2 \mathrm{H})), 7.24-7.19(\mathrm{~m}, 1 \mathrm{H}, \mathrm{ArH})$, $5.33-5.23(\mathrm{~m}, 1 \mathrm{H}, \mathrm{NCHC} \equiv \mathrm{C}), 4.58$ (ddd, $\left.J=9.2,7.6,4.7 \mathrm{~Hz}, 1 \mathrm{H}, \mathrm{NC}(\mathrm{O}) \mathrm{CH}_{2} \mathrm{NH}\right), 4.31-4.21(\mathrm{~m}$, $\left.1 \mathrm{H}, \mathrm{NC}(\mathrm{O}) \mathrm{CH}_{2} \mathrm{NH}\right), 4.00-3.97(\mathrm{~m}, 0.5 \mathrm{H}), 3.97-3.95(\mathrm{~m}, 0.5 \mathrm{H}), 3.81(\mathrm{dd}, J=16.7,5.5 \mathrm{~Hz}, 0.5 \mathrm{H})$, $3.77(\mathrm{dd}, J=16.7,5.2 \mathrm{~Hz}, 0.5 \mathrm{H}), 3.27(\mathrm{dt}, J=14.0,4.6 \mathrm{~Hz}, 1 \mathrm{H}), 3.06-2.99(\mathrm{~m}, 2 \mathrm{H}), 1.93(\mathrm{~d}, J=$ $14.2 \mathrm{~Hz}, 3 \mathrm{H}, A c-\mathrm{NH}), 1.22\left(\mathrm{t}, J=7.5 \mathrm{~Hz}, 3 \mathrm{H}, \mathrm{NC}(\mathrm{O}) \mathrm{CHCH}_{3} \mathrm{NH}\right)$. 
<smiles>CC(=O)NC(CC(=O)O)C(=O)N[C@@H](Cc1ccccc1)C(=O)NCC(=O)N1CCCC1C#Cc1ccccc1</smiles>

Following the general procedure, Ac-Asp-Phe-Gly-Pro-OH (10t) afforded 11t in more than 95\% yield (retention time 13.020).

HRMS (ESI/QTOF) m/z: [M + Na] ${ }^{+}$Calcd for $\mathrm{C}_{22} \mathrm{H}_{27} \mathrm{~N}_{4} \mathrm{O}_{8}^{-}$555.2214; Found 555.2218.

Ac-Asp-Phe-Gly-Pro-OH (10t)

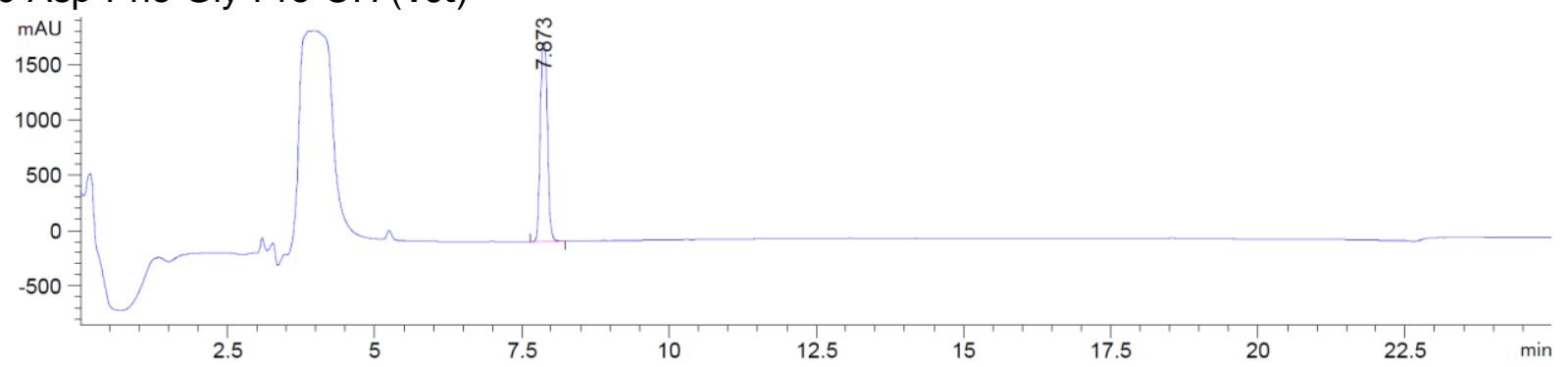

HRMS of 10t (ESI/QTOF) m/z: [M + $\left.\mathrm{H}_{-1}\right]^{-}$Calcd for $\mathrm{C}_{24} \mathrm{H}_{34} \mathrm{~N}_{7} \mathrm{O}_{6}{ }^{-}$475.1834; Found 475.1832.

HPLC-UV chromatogram at $214 \mathrm{~nm}$ of the crude reaction mixture

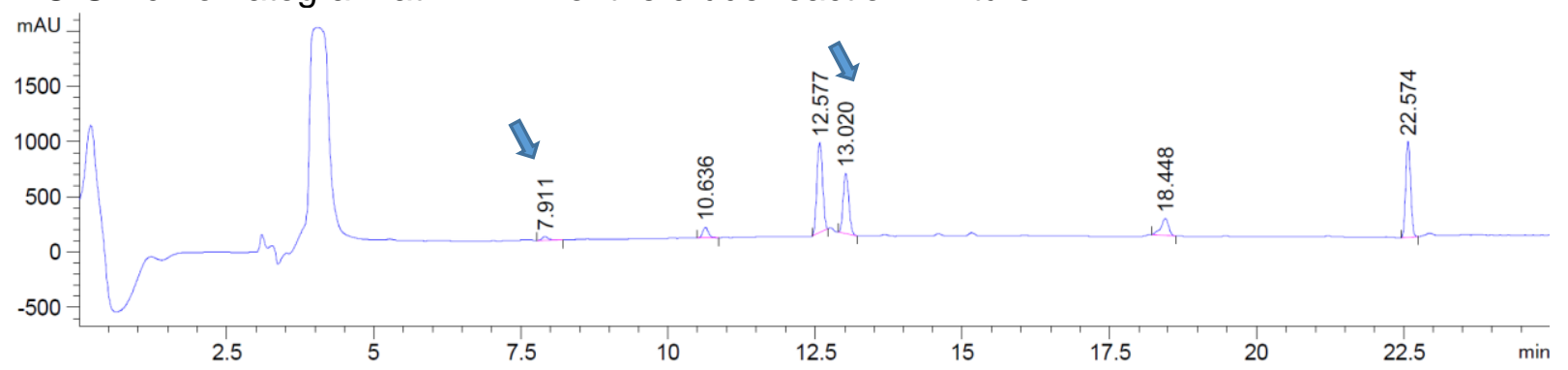

MSMS (nanochip-ESI/LTQ-Orbitrap): selected ion 533.2399. Measured $\boldsymbol{b}$ and $\boldsymbol{y}$ ions are reported in the table below.

\begin{tabular}{ccccc} 
& $\mathbf{D}$ & $\mathbf{F}$ & $\mathbf{G}$ & $\mathbf{P}^{*}$ \\
\hline $\begin{array}{c}\text { N-terminal } \\
\mathbf{b}\end{array}$ & 1 & 2 & 3 & 4 \\
\hline & - & 305.11 & 362.13 & - \\
\hline $\begin{array}{c}\text { C-terminal } \\
\mathbf{y}\end{array}$ & 4 & & & 1 \\
\hline
\end{tabular}

\section{Ac-Asp-Phe-Gly-Ala-AH (11u)}


<smiles>CC(=O)N[C@@H](CC(=O)O)C(=O)N[C@@H](Cc1ccccc1)C(=O)NCC(=O)NC(C)C#Cc1ccccc1</smiles>

Following the general procedure, Ac-Asp-Phe-Gly-Ala-OH (10u) afforded $\mathbf{1 1} \mathbf{u}$ in more than 95\% yield (retention time 12.426). The product with double alkynylation was detected at a retention time of 15.88 as less than $5 \%$ as the single alkynyation at the C-terminal position.

HRMS (nanochip-ESI/LTQ-Orbitrap) m/z: $[\mathrm{M}+\mathrm{H}]^{+}$Calcd for $\mathrm{C}_{27} \mathrm{H}_{31} \mathrm{~N}_{4} \mathrm{O}_{6}{ }^{+}$507.2238; Found 507.2235 .

Ac-Asp-Phe-Gly-Ala-OH (10u)
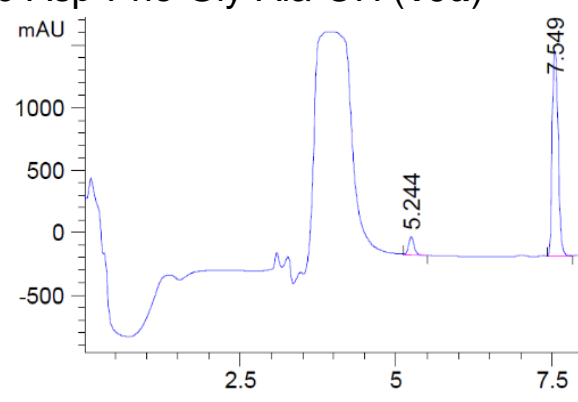

10

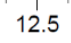

15

17.5

20

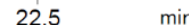

HRMS of 10u (ESI/QTOF) m/z: [M + Na] ${ }^{+}$Calcd for $\mathrm{C}_{20} \mathrm{H}_{26} \mathrm{~N}_{4} \mathrm{NaO}_{8}{ }^{+}$473.1643; Found 473.1646.

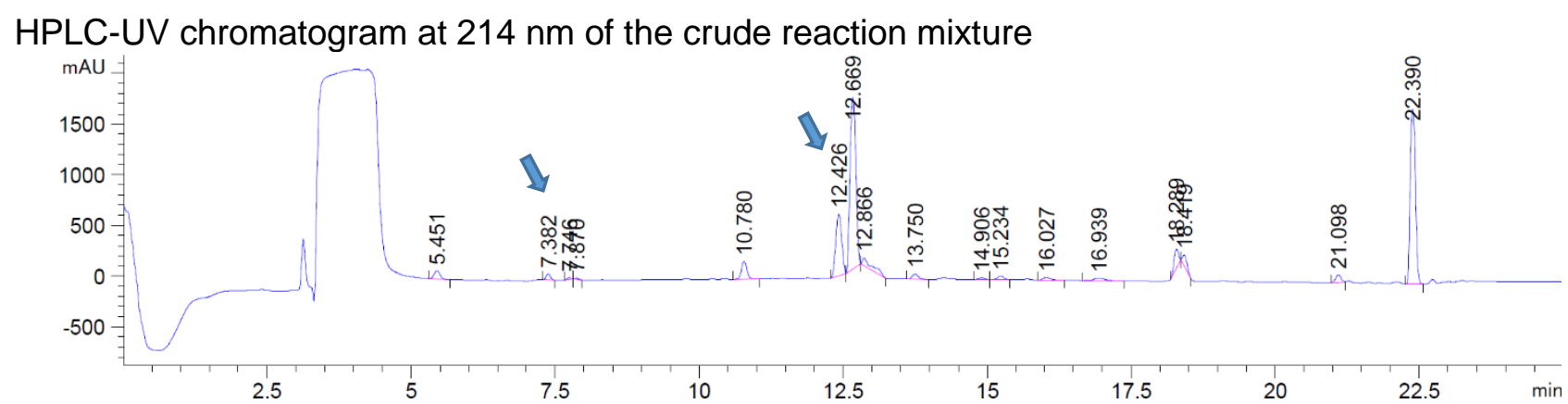

MSMS (nanochip-ESI/LTQ-Orbitrap): selected ion 507.2235. Measured $\boldsymbol{b}$ and $\boldsymbol{y}$ ions are reported in the table below.<smiles>CC(=O)NC(CC(=O)O)C(=O)N[C@@H](Cc1ccccc1)C(=O)NCC(=O)NC(C)C#CPc1ccccc1</smiles>

\begin{tabular}{ccccc} 
& D & F & G & A $^{*}$ \\
\hline $\begin{array}{c}\text { N-terminal } \\
\text { b }\end{array}$ & 1 & 2 & 3 & 4 \\
\hline C-terminal & 158.0448 & 305.1134 & 362.1348 & - \\
$\mathbf{y}$ & & & & \\
\hline
\end{tabular}


<smiles>CC(=O)NC(CC(=O)O)C(=O)N[C@@H](Cc1ccccc1)C(=O)NCC(=O)NCC#Cc1ccccc1</smiles>

Following the general procedure, Ac-Asp-Phe-Gly-Gly-OH (10v) afforded 11v in an average of $48 \%$ yield (retention time 11.503). The product with double alkynylation was detected at a retention time of 15.38 as less than $5 \%$ as the single alkynyation at the C-terminal position.

HRMS (ESI/QTOF) m/z: [M + Na] ${ }^{+}$Calcd for $\mathrm{C}_{26} \mathrm{H}_{28} \mathrm{~N}_{4} \mathrm{NaO}_{6}{ }^{+}$515.1901; Found 515.1896.

Ac-Asp-Phe-Gly-Gly-OH (10v)

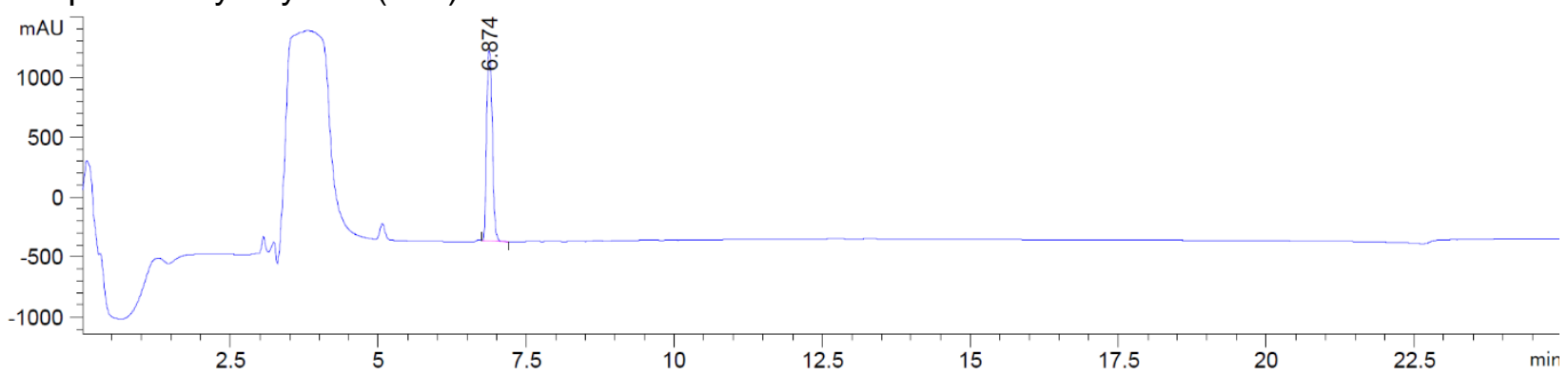

HRMS of 10v (ESI/QTOF) m/z: [M + Na] ${ }^{+}$Calcd for $\mathrm{C}_{19} \mathrm{H}_{24} \mathrm{~N}_{4} \mathrm{NaO}_{8}{ }^{+} 459.1486$; Found 459.1481.

HPLC-UV chromatogram at $214 \mathrm{~nm}$ of the crude reaction mixture

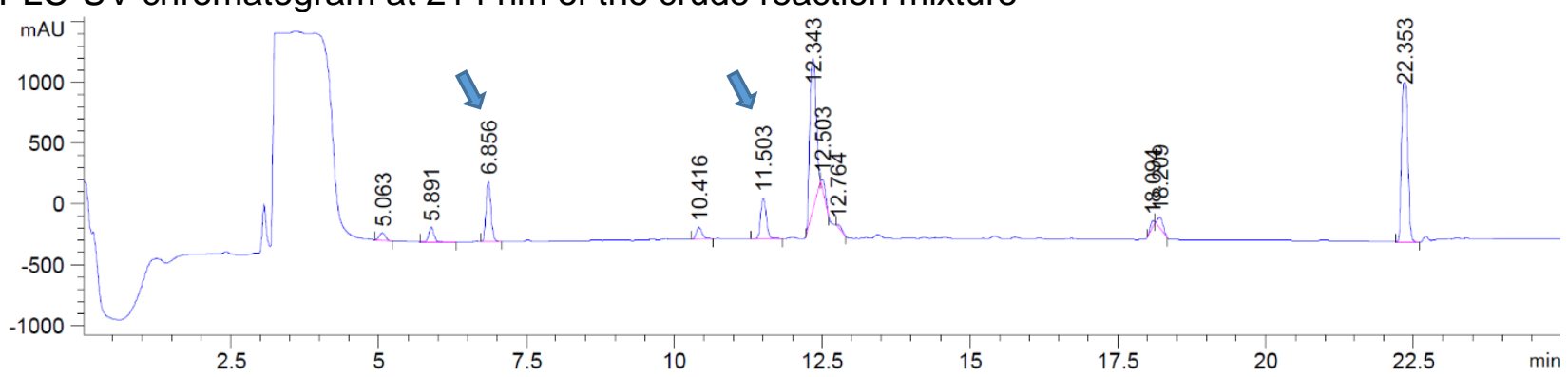

MSMS (nanochip-ESI/LTQ-Orbitrap): selected ion 493.2085. Measured $\boldsymbol{b}$ and $\boldsymbol{y}$ ions are reported in the table below.<smiles>CC(=O)NC(CC(=O)O)C(=O)N[C@@H](Cc1ccccc1)C(=O)NCC(=O)NCC#Cc1ccccc1</smiles>

\begin{tabular}{ccccc} 
& D & F & G & $\mathbf{G}^{*}$ \\
\hline $\begin{array}{c}\text { N-terminal } \\
\text { b }\end{array}$ & 1 & 2 & 3 & 4 \\
\hline
\end{tabular}




\section{Ac-Glu-Phe-Gly-Pro-AH (11w)}<smiles>CC(=O)N[C@@H](CCC(=O)O)C(=O)N[C@@H](Cc1ccccc1)C(=O)NCC(=O)N1CCCC1C#Cc1ccccc1</smiles>

Following the general procedure, Ac-Glu-Phe-Gly-Pro-OH (10w) afforded $\mathbf{1 1 w}$ in more than 95\% yield (retention time 13.017). The product with double alkynylation was not detected.

HRMS (nanochip-ESI/LTQ-Orbitrap) m/z: $[\mathrm{M}+\mathrm{H}]^{+}$Calcd for $\mathrm{C}_{30} \mathrm{H}_{35} \mathrm{~N}_{4} \mathrm{O}_{6}+547.2551$; Found 547.2547.

Ac-Glu-Phe-Gly-Pro-OH (10w)

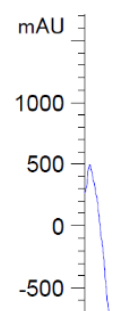

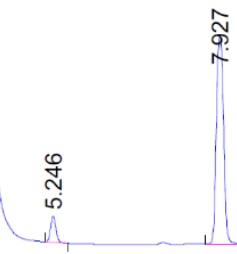

(5)

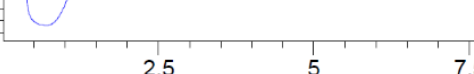

12.5

15

17.5

20

$225 \quad \min$

HRMS of 10w (ESI/QTOF) m/z: [M + Na] ${ }^{+}$Calcd for $\mathrm{C}_{23} \mathrm{H}_{30} \mathrm{~N}_{4} \mathrm{NaO}_{8}{ }^{+}$513.1956; Found 513.1958.

HPLC-UV chromatogram at $214 \mathrm{~nm}$ of the crude reaction mixture

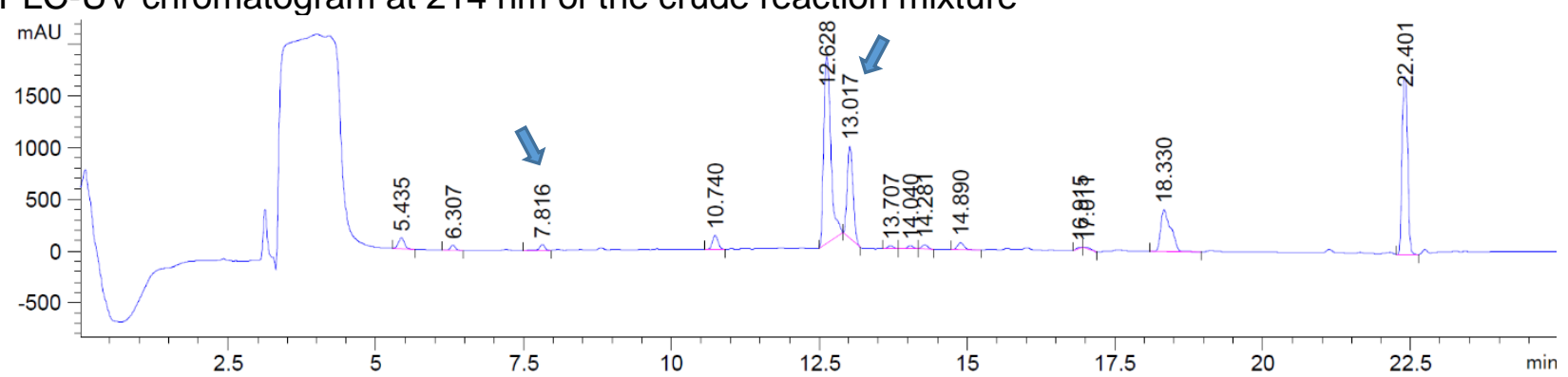

MSMS (nanochip-ESI/LTQ-Orbitrap): selected ion 547.3604. Measured $\boldsymbol{b}$ and $\boldsymbol{y}$ ions are reported in the table below.<smiles>CC(=O)N[C@@H](CC(C)C(=O)O)C(=O)N[C@@H](Cc1ccccc1)C(=O)NCC(=O)N1CCCC1C#CC#Cc1ccccc1</smiles>

$\mathbf{E}$

$\mathbf{F}$

G

$\mathbf{P}^{*}$ 


\begin{tabular}{ccccc}
\hline $\begin{array}{c}\text { N-terminal } \\
\mathbf{b}\end{array}$ & 1 & 2 & 3 & 4 \\
\hline & 172.0606 & 319.1288 & 376.1500 & - \\
\hline $\begin{array}{c}\text { C-terminal } \\
\mathbf{y}\end{array}$ & 4 & & & 1 \\
\hline
\end{tabular}

\section{Ac-Glu-Phe-Gly-Gly-AH (11x)}<smiles>CC(=O)N[C@@H](CCC(=O)O)C(=O)N[C@@H](Cc1ccccc1)C(=O)NCC(=O)NCC#Cc1ccccc1</smiles>

Following the general procedure, Ac-Glu-Phe-Gly-Gly-OH (10x) afforded 11x in an average of $75 \%$ yield (retention time 11.738). The product with double alkynylation was only detected in HRMS of the crude, not in the HPLC chromatogram.

HRMS (ESI/QTOF) m/z: [M + Na] ${ }^{+}$Calcd for $\mathrm{C}_{27} \mathrm{H}_{30} \mathrm{~N}_{4} \mathrm{NaO}_{6}{ }^{+}$529.2058; Found 529.2059.

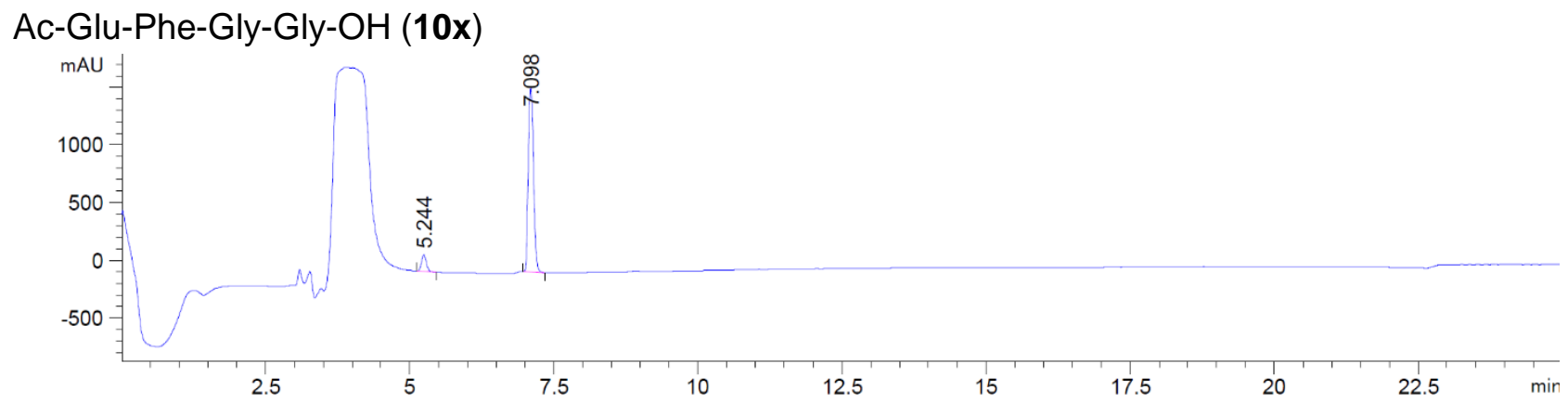

HRMS of 10x (ESI/QTOF) m/z: [M + Na] ${ }^{+}$Calcd for $\mathrm{C}_{20} \mathrm{H}_{26} \mathrm{~N}_{4} \mathrm{NaO}_{8}{ }^{+}$473.1643; Found 473.1648.

HPLC-UV chromatogram at $214 \mathrm{~nm}$ of the crude reaction mixture

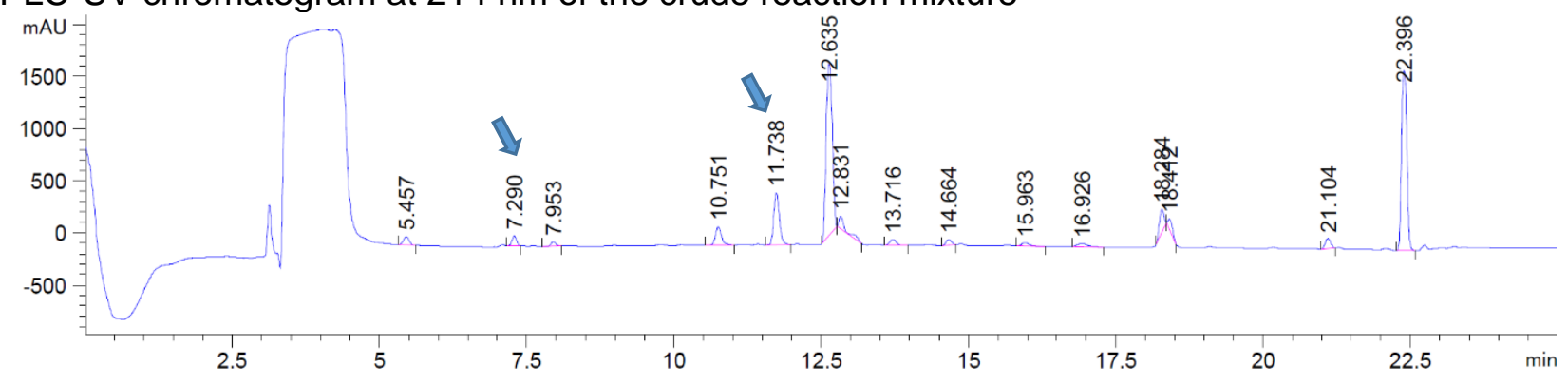

MSMS (nanochip-ESI/LTQ-Orbitrap): selected ion 507.2235. Measured $\boldsymbol{b}$ and $\boldsymbol{y}$ ions are reported in the table below. 


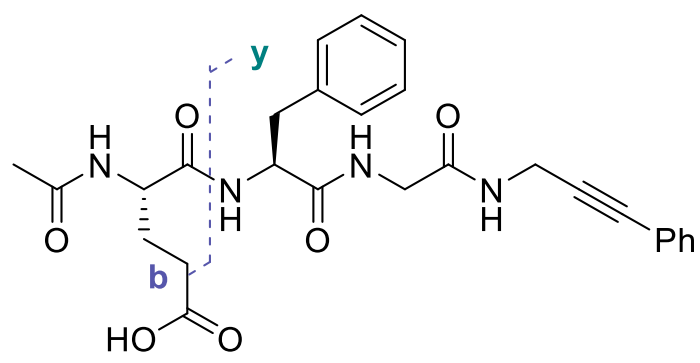

\begin{tabular}{ccccc} 
& $\mathbf{E}$ & $\mathbf{F}$ & $\mathbf{G}$ & $\mathbf{G}^{*}$ \\
\hline $\begin{array}{c}\text { N-terminal } \\
\mathbf{b}\end{array}$ & 1 & 2 & 3 & 4 \\
\hline & 172.0605 & 319.1289 & 376.1503 & - \\
\hline $\begin{array}{c}\text { C-terminal } \\
\mathbf{y}\end{array}$ & 4 & & & \\
\hline
\end{tabular}




\subsection{Scope on Gly-Arg-Gly-Asp-Asn-Pro-OH}

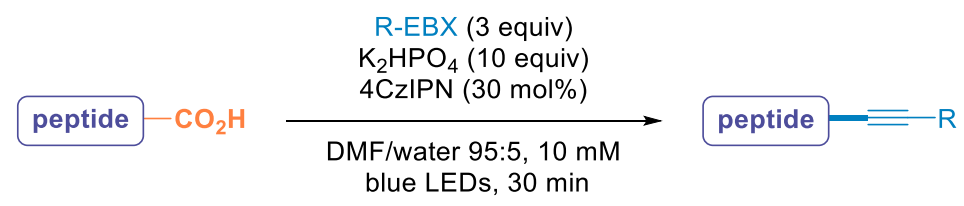

\section{General procedure 5 for the decarboxylative alkynylation of Gly-Arg-Gly-Asp-Asn-Pro-OH}

A $20 \mathrm{mM}$ solution of the peptide in non-degassed DMF (50 $\mu \mathrm{L}, 1.0 \mu \mathrm{mol})$, a $60 \mu \mathrm{M}$ solution of R-EBX (1) in DMF (25 $\mu \mathrm{L}, 3.0 \mu \mathrm{mol}, 3.0$ equiv), a $15 \mu \mathrm{M}$ solution of $4 \mathrm{CzIPN}(\mathbf{2 a})$ in DMF $(20 \mu \mathrm{L}, 0.30 \mu \mathrm{mol}$, $30 \mathrm{~mol} \%)$ and a $2 \mathrm{M}$ solution of $\mathrm{K}_{2} \mathrm{HPO}_{4}$ in milli-Q purified water $(5 \mu \mathrm{L}, 10 \mu \mathrm{mol}, 10$ equiv) were placed into a vial. The vial was then caped and degassed by bubbling with argon for 1-2 min and the mixture was irradiated using blue light LEDs for 30 min at RT.

At the end of the reaction, the crude was diluted with $3 x$ the volume of MeCN/water 1:1 and injected in RP-HPLC. The yields were determined as the ratio of $A_{\text {prod }} / A_{\text {total }}$ where $A_{\text {prod }}=$ area in $m A U$ of the product peak and $A_{\text {total }}=$ area in $\mathrm{mAU}$ of all peptides products (product, starting material, and sideproducts if present).

Reported results are an average of 3 independent trials.

The products are described as peptide-AR with $\mathrm{R}$ standing for the substitution on the aryl ring.

Reference HPLC-UV chromatograms of reagents at $214 \mathrm{~nm}$ (lodobenzoic acid, and 4CzIPN in section 0 )

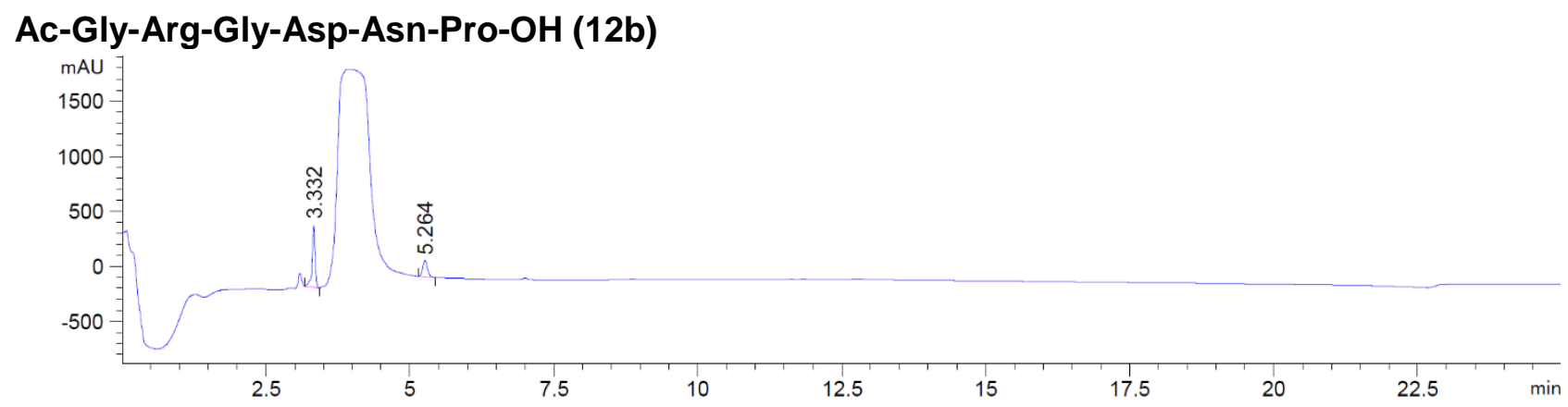

HRMS (ESI/QTOF) m/z: [M + $\left.\mathrm{H}_{-1}\right]^{-}$Calcd for $\mathrm{C}_{25} \mathrm{H}_{39} \mathrm{~N}_{10} \mathrm{O}_{11}{ }^{-}$655.2805; Found 655.2802.

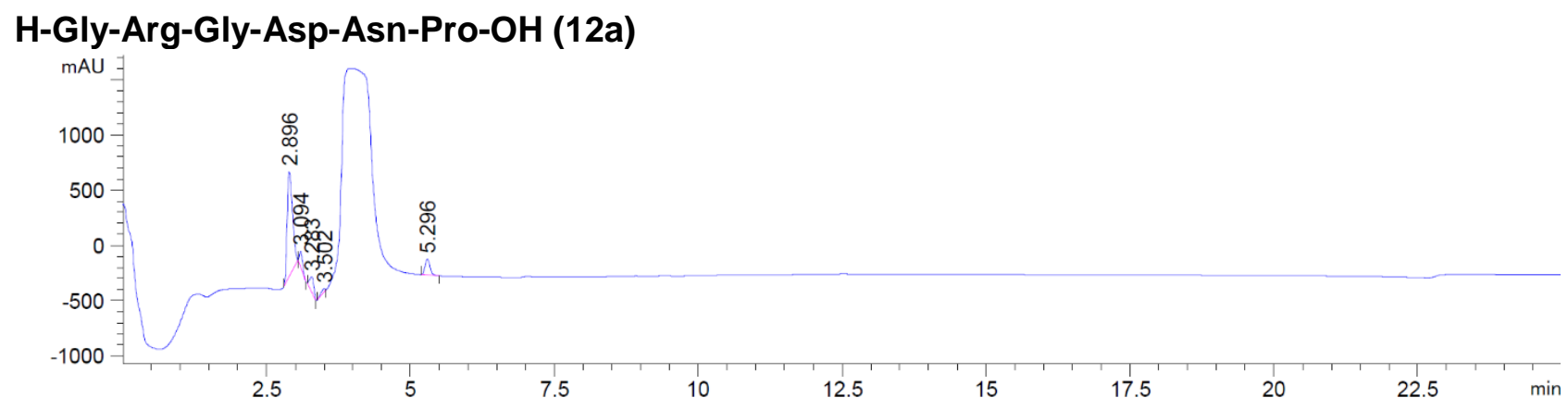


1-[4-Bromophenylethynyl]-1,2-benziodoxol-3(1H)-one (pBr-Ph-EBX, 1d)

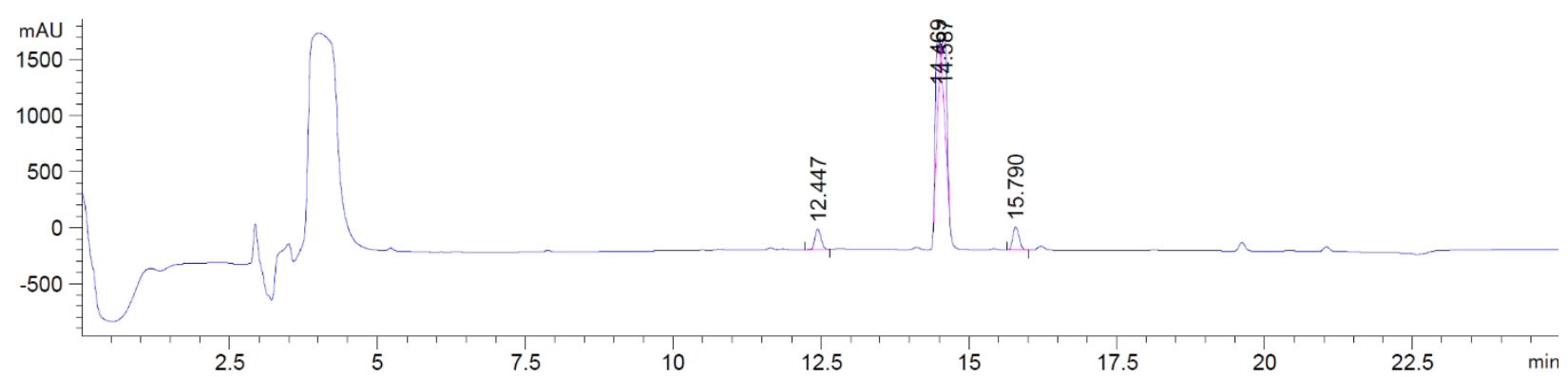

1-((4-(Prop-2-yn-1-yl-benzoate)ethynyl-1,2-benziodoxol-3(1H)-one $\left(\mathrm{N}_{3}\right.$-Ar-EBX, 1j)

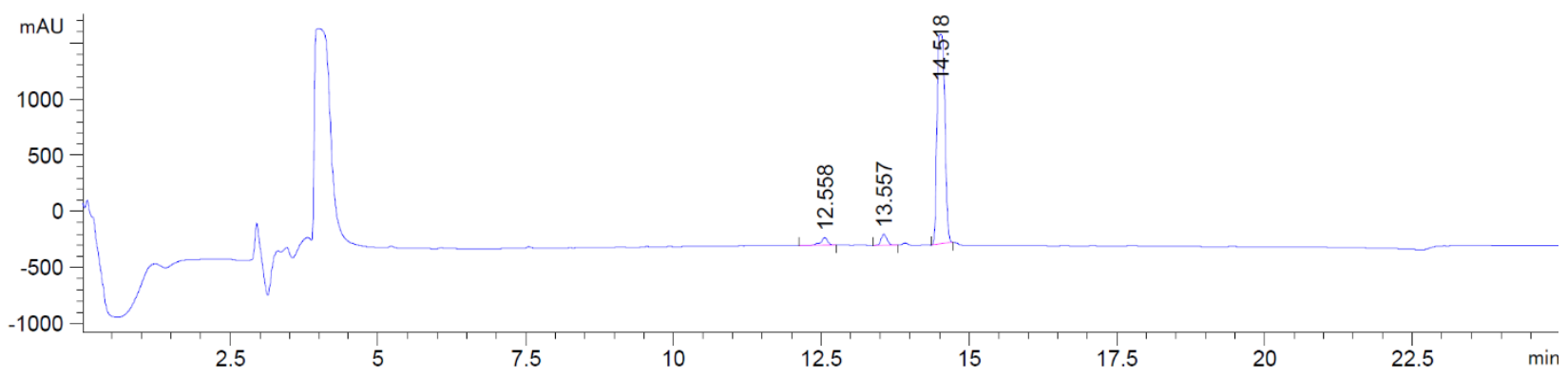

1-((4-Formylphenyl)ethynyl)-1,2-benziodoxol-3(1H)-one (CHO-Ph-EBX, 1e)

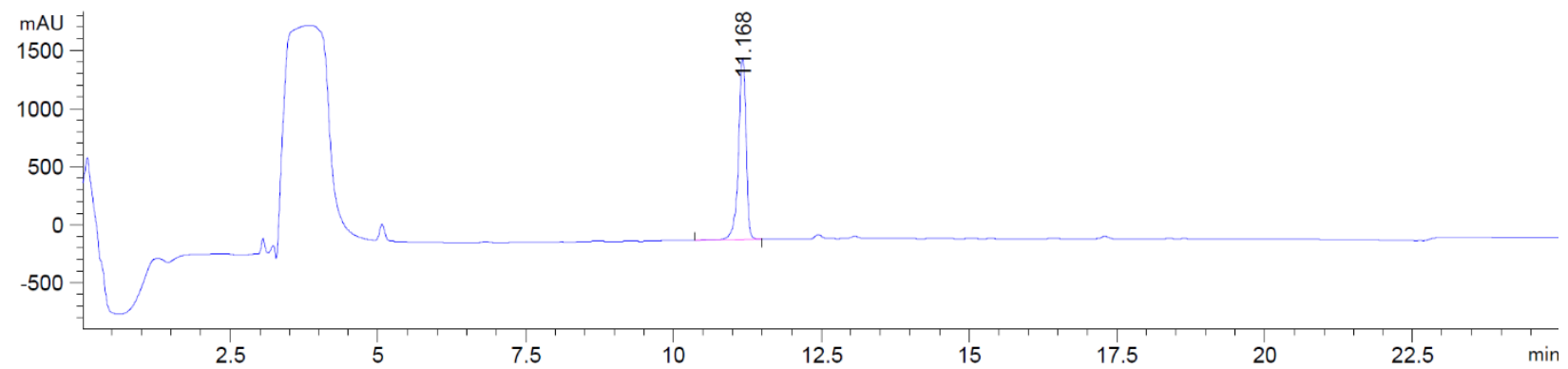


<smiles>CC(=O)NCC(=O)N[C@@H](CCCNC(=N)N)C(=O)NCC(=O)N[C@@H](CC(=O)O)C(=O)N[C@@H](CC(N)=O)C(=O)N1CCCC1C#Cc1ccc(C(=O)OCCCN)cc1</smiles>

Following the general procedure, Ac-Gly-Arg-Gly-Asp-Asn-Pro-OH (12a) afforded 13a in quantitative yield (retention time 9.874).

HRMS (nanochip-ESI/LTQ-Orbitrap) $\mathrm{m} / \mathrm{z}$ : $[\mathrm{M}+\mathrm{H}]^{+}$Calcd for $\mathrm{C}_{36} \mathrm{H}_{50} \mathrm{~N}_{13} \mathrm{O}_{11}+840.3747$; Found 840.3739 .

HPLC-UV chromatogram at $214 \mathrm{~nm}$ :

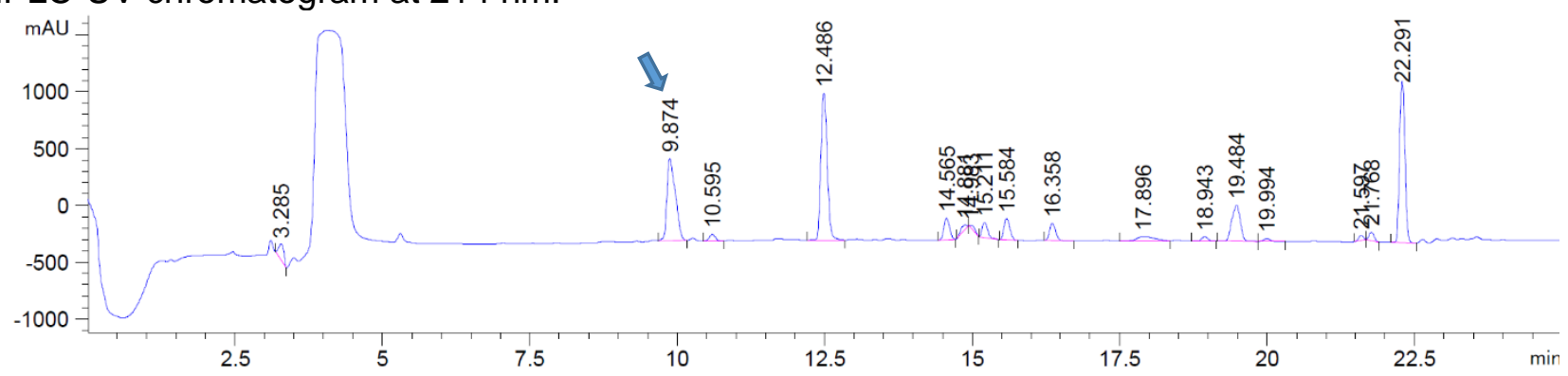

MSMS (nanochip-ESI/LTQ-Orbitrap): selected ion 840.3739. Identified $\boldsymbol{b}$ ions are reported in the table below. $\boldsymbol{y}$ ions could not be identified due to non-controlled fragmentation.

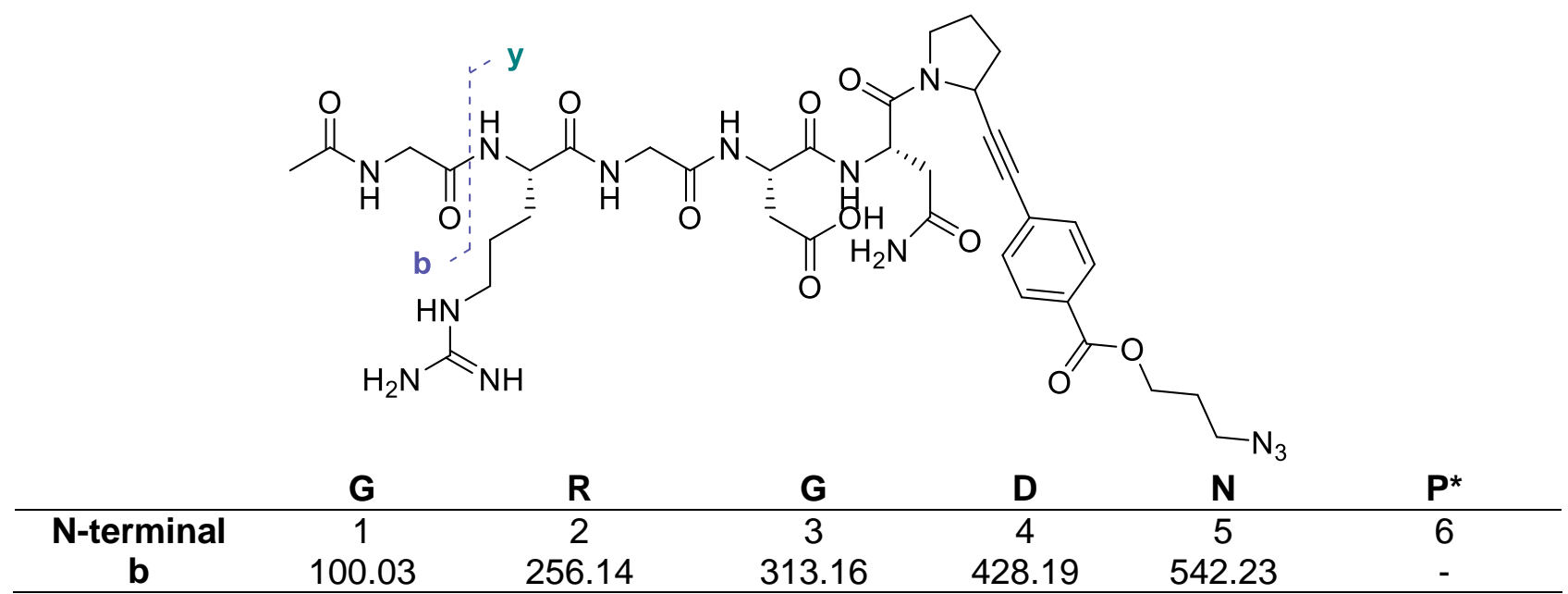




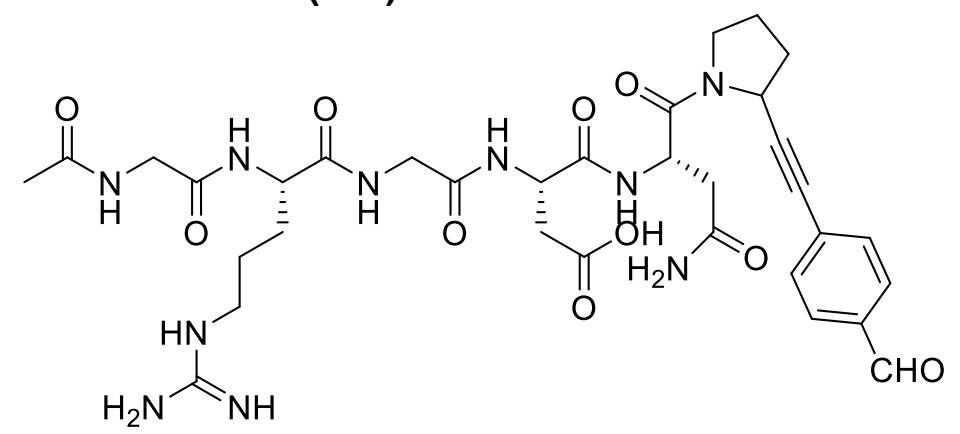

Following the general procedure, Ac-Gly-Arg-Gly-Asp-Asn-Pro-OH (12a) afforded 13b in quantitative yield (retention time 7.594).

HRMS (nanochip-ESI/LTQ-Orbitrap) $\mathrm{m} / \mathrm{z}$ : $[\mathrm{M}+\mathrm{H}]^{+}$Calcd for $\mathrm{C}_{33} \mathrm{H}_{45} \mathrm{~N}_{10} \mathrm{O}_{10}{ }^{+} 741.3315$; Found 741.3302.

HPLC-UV chromatogram at $214 \mathrm{~nm}$ :

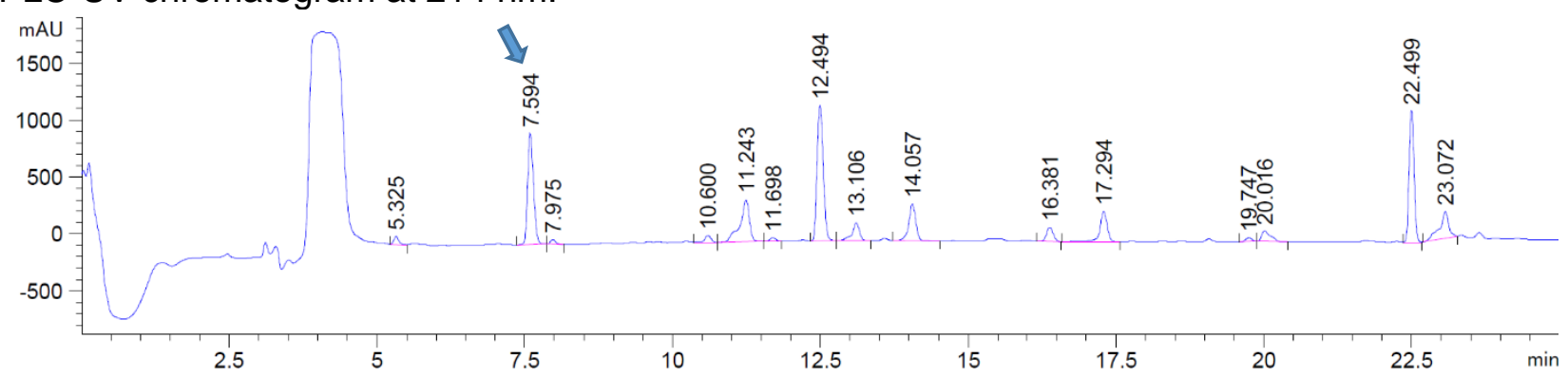

MSMS (nanochip-ESI/LTQ-Orbitrap): selected ion 741.3302. Identified $\boldsymbol{b}$ and $\boldsymbol{y}$ ions are reported in the table below.

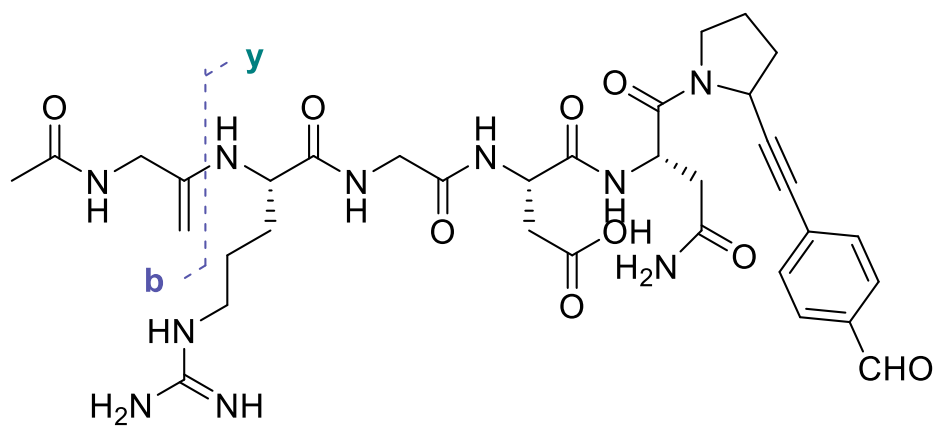

\begin{tabular}{ccccccc} 
& $\mathbf{G}$ & $\mathbf{R}$ & $\mathbf{G}$ & $\mathbf{D}$ & $\mathbf{N}$ & $\mathbf{P}^{*}$ \\
\hline $\begin{array}{c}\text { N-terminal } \\
\mathbf{b}\end{array}$ & 1 & 2 & 3 & 4 & 5 & 6 \\
\hline & 100.03 & 256.14 & 313.16 & 428.19 & 542.23 & - \\
\hline $\begin{array}{c}\text { C-terminal } \\
\mathbf{y}\end{array}$ & 6 & 5 & 4 & 3 & 2 & 1 \\
\hline
\end{tabular}




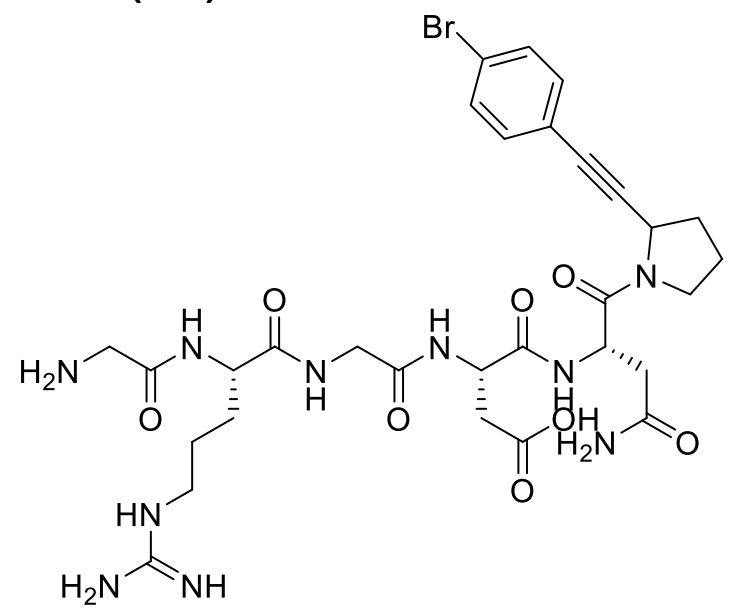

Following the general procedure, H-Gly-Arg-Gly-Asp-Asn-Pro-OH (12b) afforded 13c in 52\% yield (retention time 8.326).

HRMS (ESI/QTOF) m/z: [M + H] ${ }^{+}$Calcd for $\mathrm{C}_{30} \mathrm{H}_{42}{ }^{79} \mathrm{BrN}_{10} \mathrm{O}_{8}{ }^{+} 749.2365$, 751.2345; Found 749.2350, 751.2342.

HPLC-UV chromatogram at $214 \mathrm{~nm}$ :

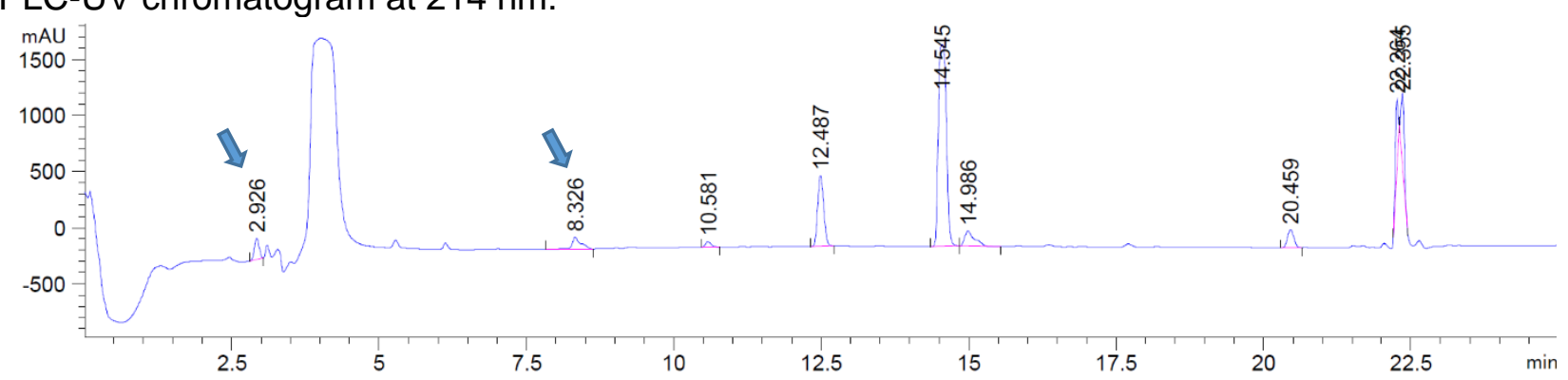

MSMS (nanochip-ESI/LTQ-Orbitrap): selected ion 749.2350. Identified $\boldsymbol{b}$ and $\boldsymbol{y}$ ions are reported in the table below.<smiles>N=C(N)NCCC[C@H](NC(=O)CN)C(=O)NCC(=O)N[C@@H](CC(=O)O)C(=O)N[C@@H](CC(N)=O)C(=O)N1CCCC1C#Cc1ccc(Br)cc1</smiles>

\begin{tabular}{ccccccc} 
& $\mathbf{G}$ & $\mathbf{R}$ & $\mathbf{G}$ & $\mathbf{D}$ & $\mathbf{N}$ & $\mathbf{P}^{*}$ \\
\hline $\begin{array}{c}\text { N-terminal } \\
\mathbf{b}\end{array}$ & 1 & 2 & 3 & 4 & 5 & 6 \\
\hline & - & 214.13 & 271.15 & 386.18 & 500.22 & - \\
\hline $\begin{array}{c}\text { C-terminal } \\
\mathbf{y}\end{array}$ & 6 & 5 & 4 & 3 & 2 & 1 \\
\hline
\end{tabular}


<smiles>N=C(N)NCCC[C@H](NC(=O)CN)C(=O)NCC(=O)N[C@@H](CC(=O)O)C(=O)N[C@@H](CC(N)=O)C(=O)N1CCCC1C#Cc1ccc(C(=O)OCCCN)cc1</smiles>

Following the general procedure, H-Gly-Arg-Gly-Asp-Asn-Pro-OH (12b) afforded 13c in 57\% yield (retention time 8.921).

HRMS (QTOF) m/z: [M + H] $]^{+}$Calcd for $\mathrm{C}_{34} \mathrm{H}_{48} \mathrm{~N}_{13} \mathrm{O}_{10}{ }^{+}$798.3642; Found 798.3640.

HPLC-UV chromatogram at $214 \mathrm{~nm}$ :

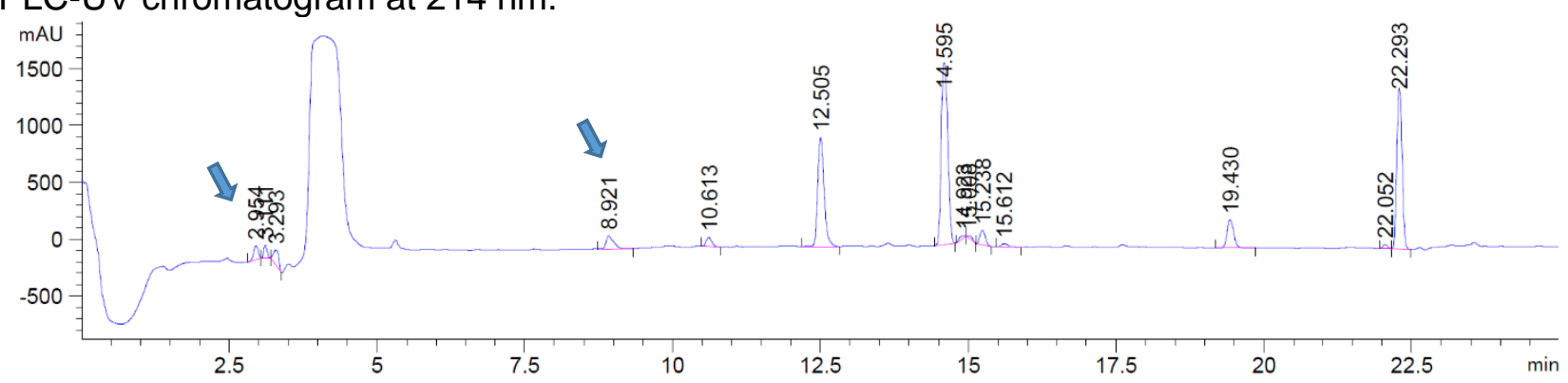

MSMS (nanochip-ESI/LTQ-Orbitrap): selected ion 798.3640. Identified $\boldsymbol{b}$ ions are reported in the table below. $\boldsymbol{y}$ ions could not be identified due to non-controlled fragmentation.<smiles>N=C(N)CCC[C@H](NC(=O)CN)C(=O)NCC(=O)N[C@@H](CC(=O)O)C(=O)N[C@@H](CC(N)=O)C(=O)N1CCCC1C#Cc1ccc(C(=O)OCCCN)cc1</smiles>

\begin{tabular}{ccccccc} 
& G & $\mathbf{R}$ & $\mathbf{G}$ & $\mathbf{D}$ & $\mathbf{N}$ & $\mathbf{P}^{*}$ \\
\hline N-terminal & 1 & 2 & 3 & 4 & 5 & 6 \\
b & - & 214.12 & 271.15 & 386.17 & 500.22 & - \\
\hline
\end{tabular}




\section{Crystal structures}

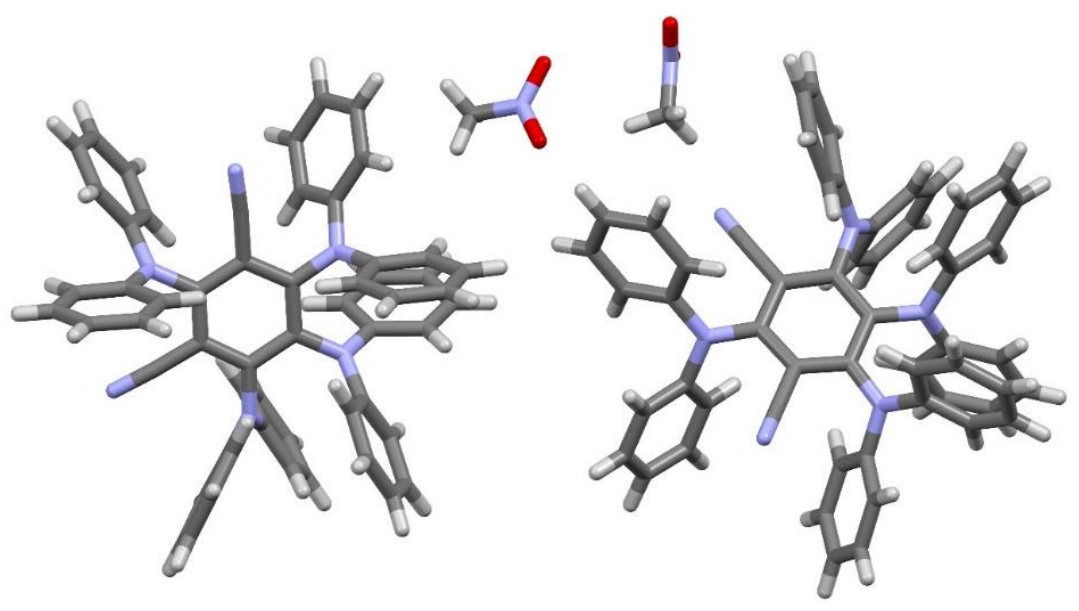

A single crystal was grown by slow diffusion of a solution of $\mathbf{3 a}$ in nitromethane. Supplementary crystallographic data for this compound have been deposited at Cambridge Crystallographic Data Centre (CCDC 1879098) and can be obtained free of charge via www.ccdc.cam.ac.uk/data_request/cif.

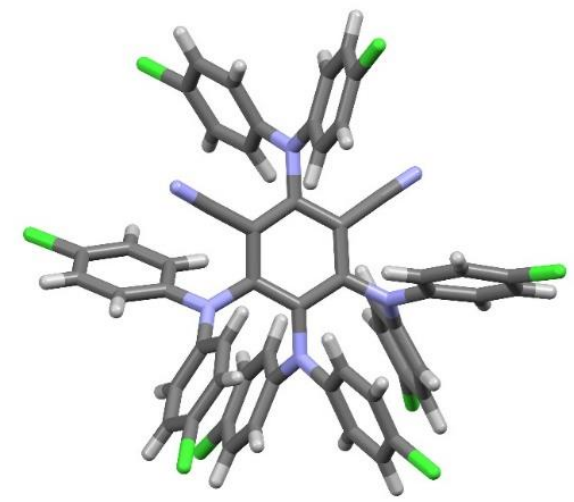

A single crystal was grown by slow diffusion of a solution of $\mathbf{3 b}$ in $\mathrm{CDCl}_{3}$. Supplementary crystallographic data for this compound have been deposited at Cambridge Crystallographic Data Centre (CCDC 1879097) and can be obtained free of charge via www.ccdc.cam.ac.uk/data_request/cif. 


\section{Bibliography}

[1] J. P. Brand, C. Chevalley, R. Scopelliti, J. Waser, Chem. Eur. J. 2012, 18, 5655-5666.

[2] C. J. Helal, P. A. Magriotis, E. J. Corey, J. Am. Chem. Soc. 1996, 118, 10938-10939.

[3] J. P. Brand, J. Waser, Angew. Chem. Int. Ed. 2010, 49, 7304-7307.

[4] B. Lu, J. Wu, N. Yoshikai, J. Am. Chem. Soc. 2014, 136, 11598-11601.

[5] K. Jia, F. Zhang, H. Huang, Y. Chen, J. Am. Chem. Soc. 2016, 138, 1514-1517.

[6] F. Le Vaillant, T. Courant, J. Waser, Angew. Chem. Int. Ed. 2015, 54, 11200-11204.

[7] H. Huang, G. Zhang, L. Gong, S. Zhang, Y. Chen, J. Am. Chem. Soc. 2014, 136, 2280-2283.

[8] Y. S. Feng, C. Q. Xie, W. L. Qiao, H. J. Xu, Org. Lett. 2013, 15, 936-939.

[9] R. J. Rahaim, J. T. Shaw, J. Org. Chem. 2008, 73, 2912-2915.

[10] F. Le Vaillant, M. Garreau, S. Nicolai, G. Gryn'Ova, C. Corminboeuf, J. Waser, Chem. Sci. 2018, 9, 5883-5889.

[11] E. Stridfeldt, A. Seemann, M. J. Bouma, C. Dey, A. Ertan, B. Olofsson, Chem. Eur. J. 2016, 22, 16066-16070.

[12] H. Uoyama, K. Goushi, K. Shizu, H. Nomura, C. Adachi, Nature 2012, 492, 234-238.

[13] A. Kretzschmar, C. Patze, S. T. Schwaebel, U. H. F. Bunz, J. Org. Chem. 2015, 80, 9126-9131.

[14] R. Ishimatsu, T. Edura, C. Adachi, K. Nakano, T. Imato, Chem. Eur. J. 2016, 22, 48894898.

[15] M. L. Louillat, F. W. Patureau, Org. Lett. 2013, 15, 164-167.

[16] E. Speckmeier, T. Fischer, K. Zeitler, J. Am. Chem. Soc. 2018, 140, 15353-15365.

[17] J. Luo, J. Zhang, ACS Catal. 2016, 6, 873-877.

[18] D. Maiti, B. P. Fors, J. L. Henderson, Y. Nakamura, S. L. Buchwald, Chem. Sci. 2011, 2, 57-68.

[19] R. Ishimatsu, S. Matsunami, T. Kasahara, J. Mizuno, T. Edura, C. Adachi, K. Nakano, T. Imato, Angew. Chem. Int. Ed. 2014, 53, 6993-6996.

[20] N. Elgrishi, K. J. Rountree, B. D. McCarthy, E. S. Rountree, T. T. Eisenhart, J. L. Dempsey, J. Chem. Educ. 2018, 197-206.

[21] N. A. Romero, D. A. Nicewicz, Chem. Rev. 2016, 116, 10075-10166. 


\section{NMR spectra}

${ }^{1} \mathrm{H}-\mathrm{NMR}$ (400 MHz, Chloroform-d)

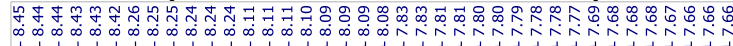
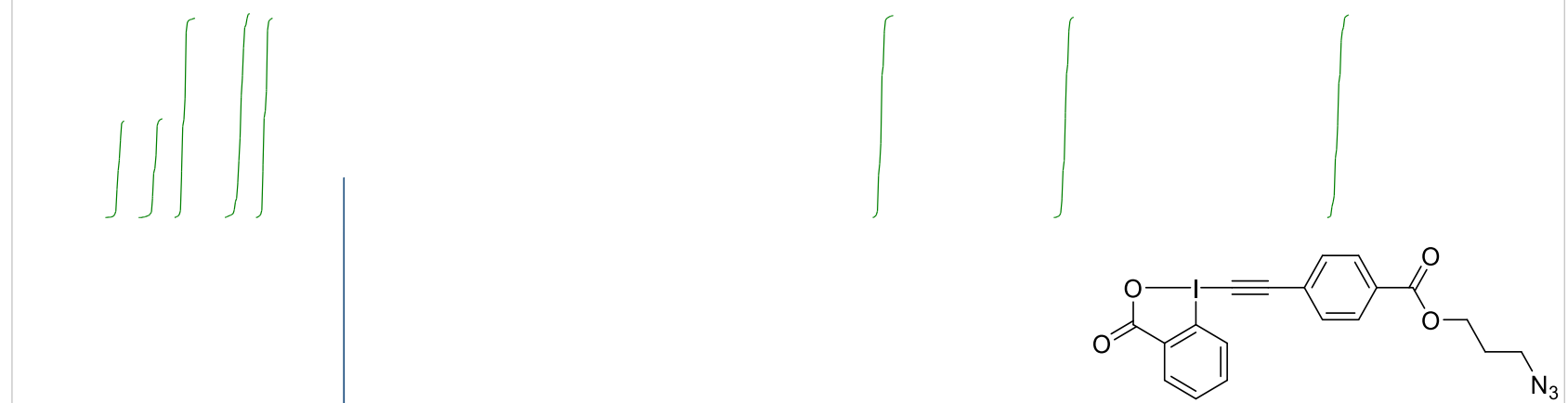

$1 j$
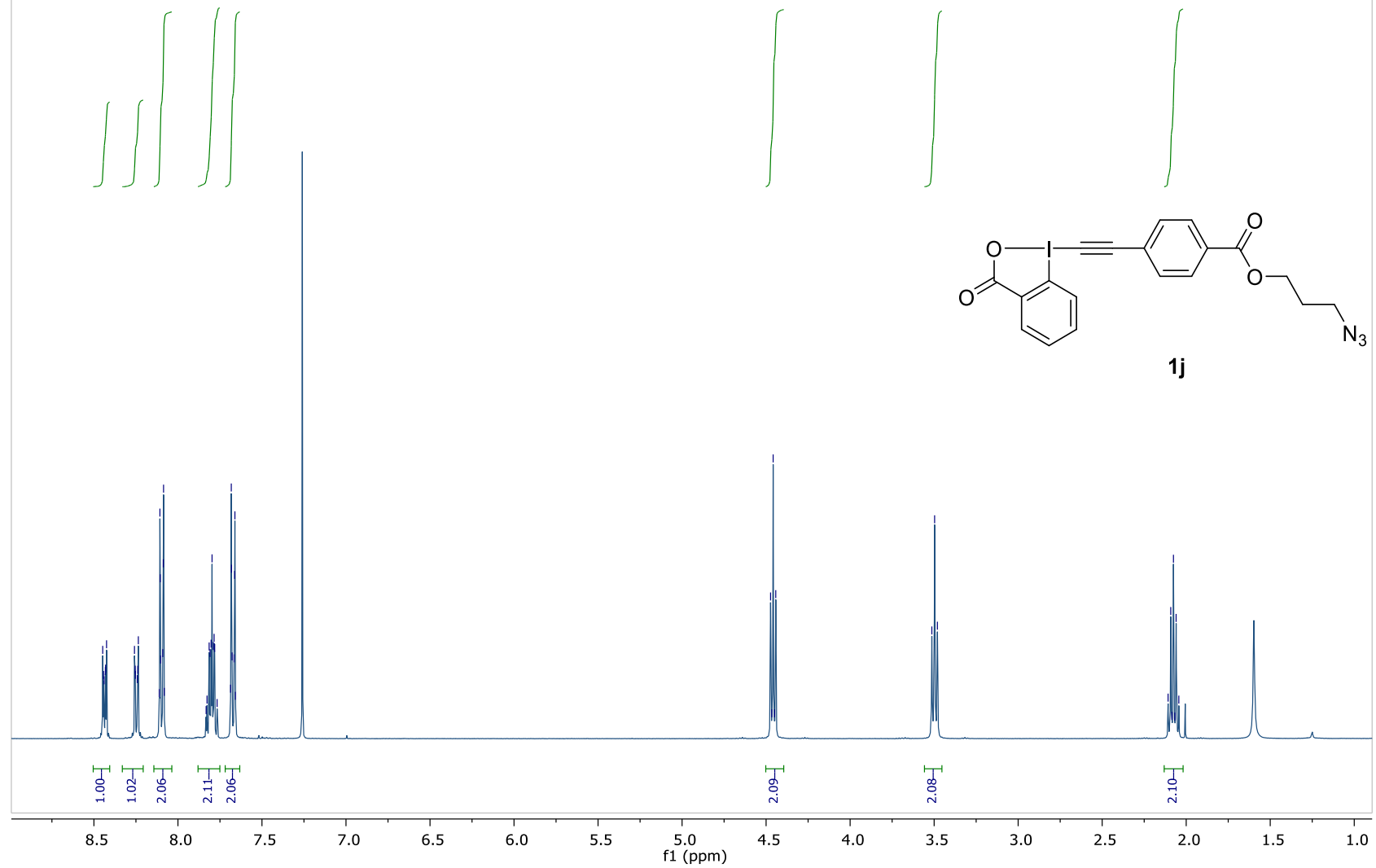

${ }^{13} \mathrm{C}-\mathrm{NMR}$ (101 MHz, Chloroform-d)

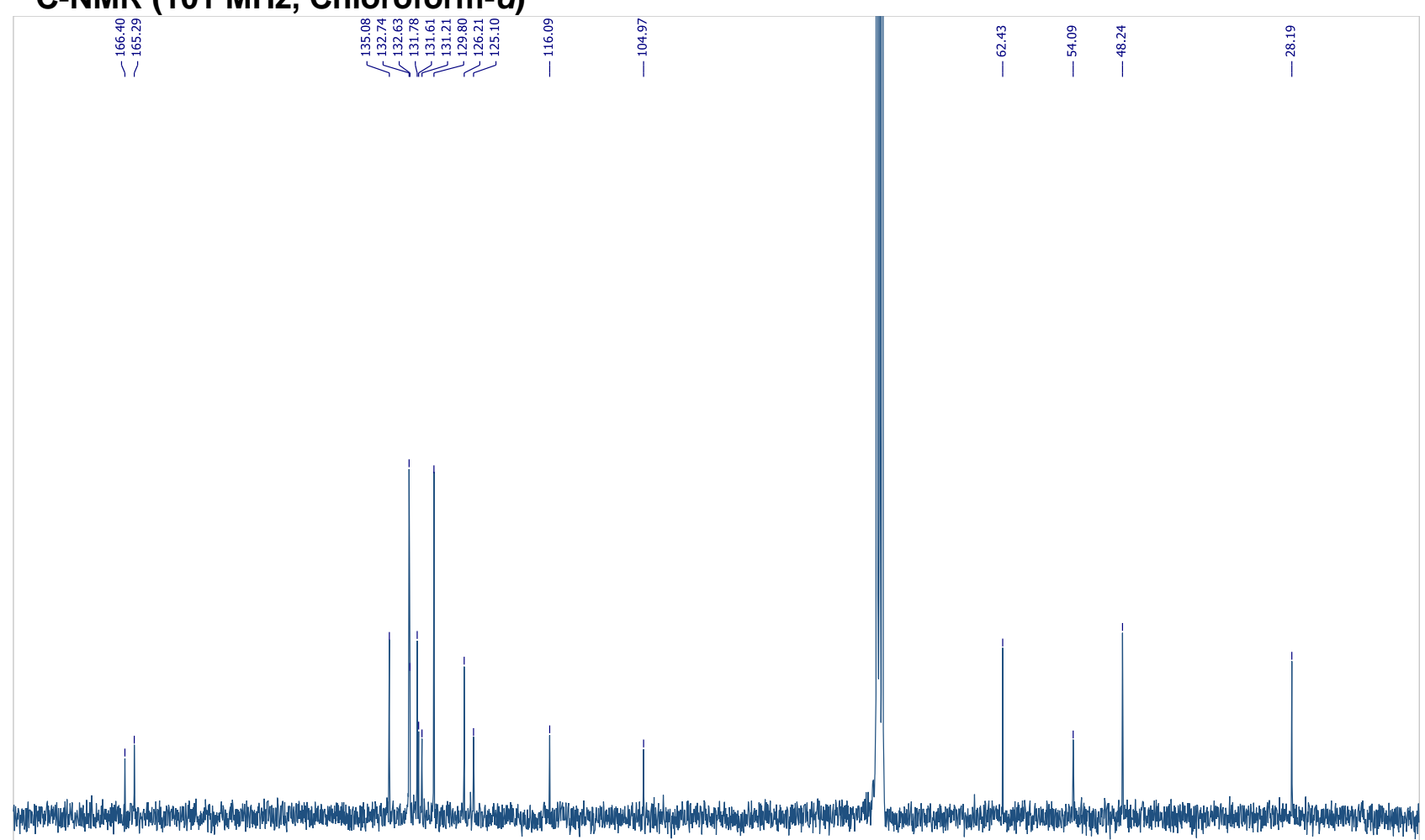

$170 \quad 160$

$150 \quad 140$

${ }_{\mathrm{f} 1(\mathrm{ppm})}^{100} 90$

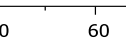

50 


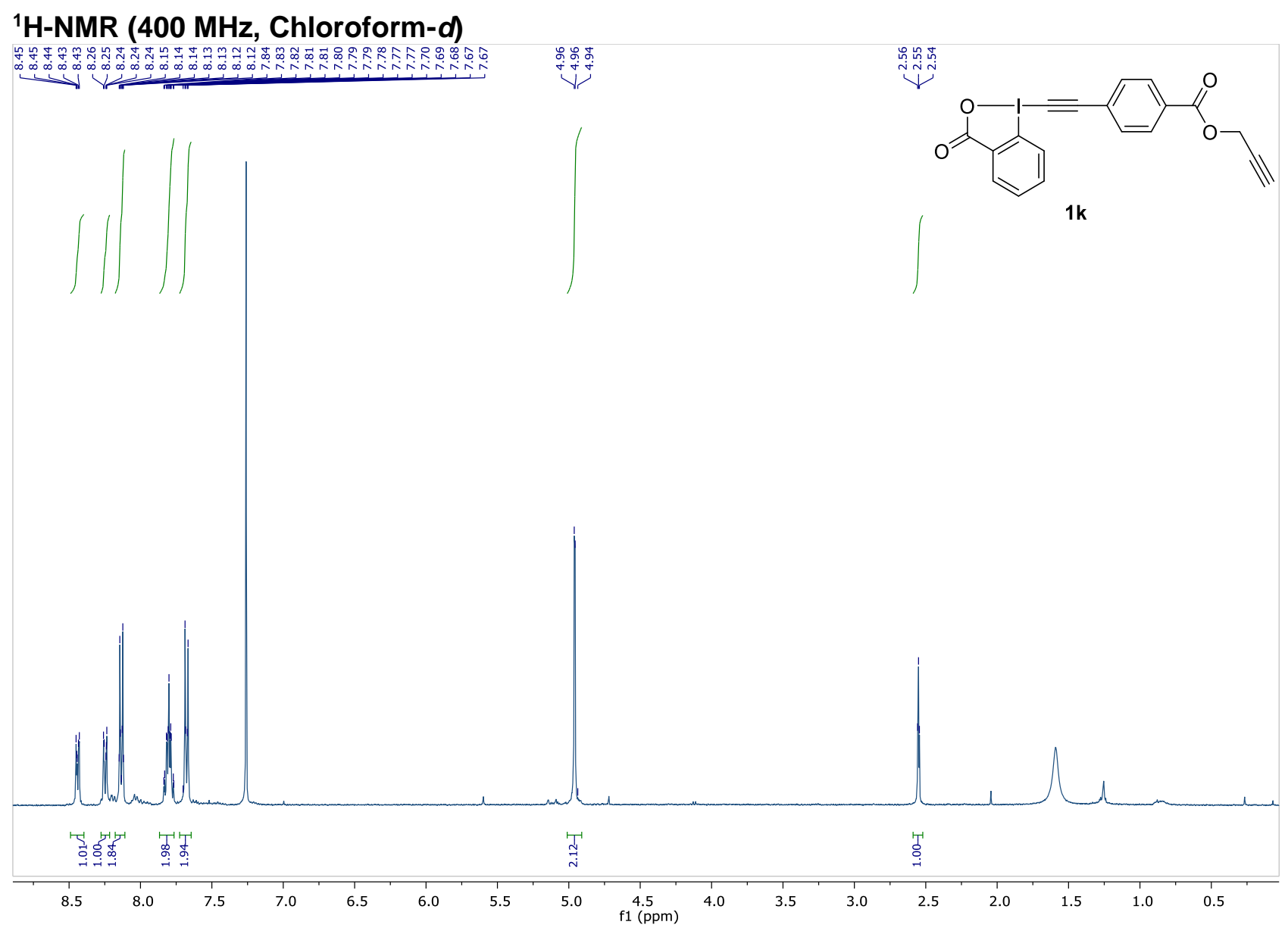

${ }^{13}$ C-NMR (101 MHz, Chloroform- $d$ )

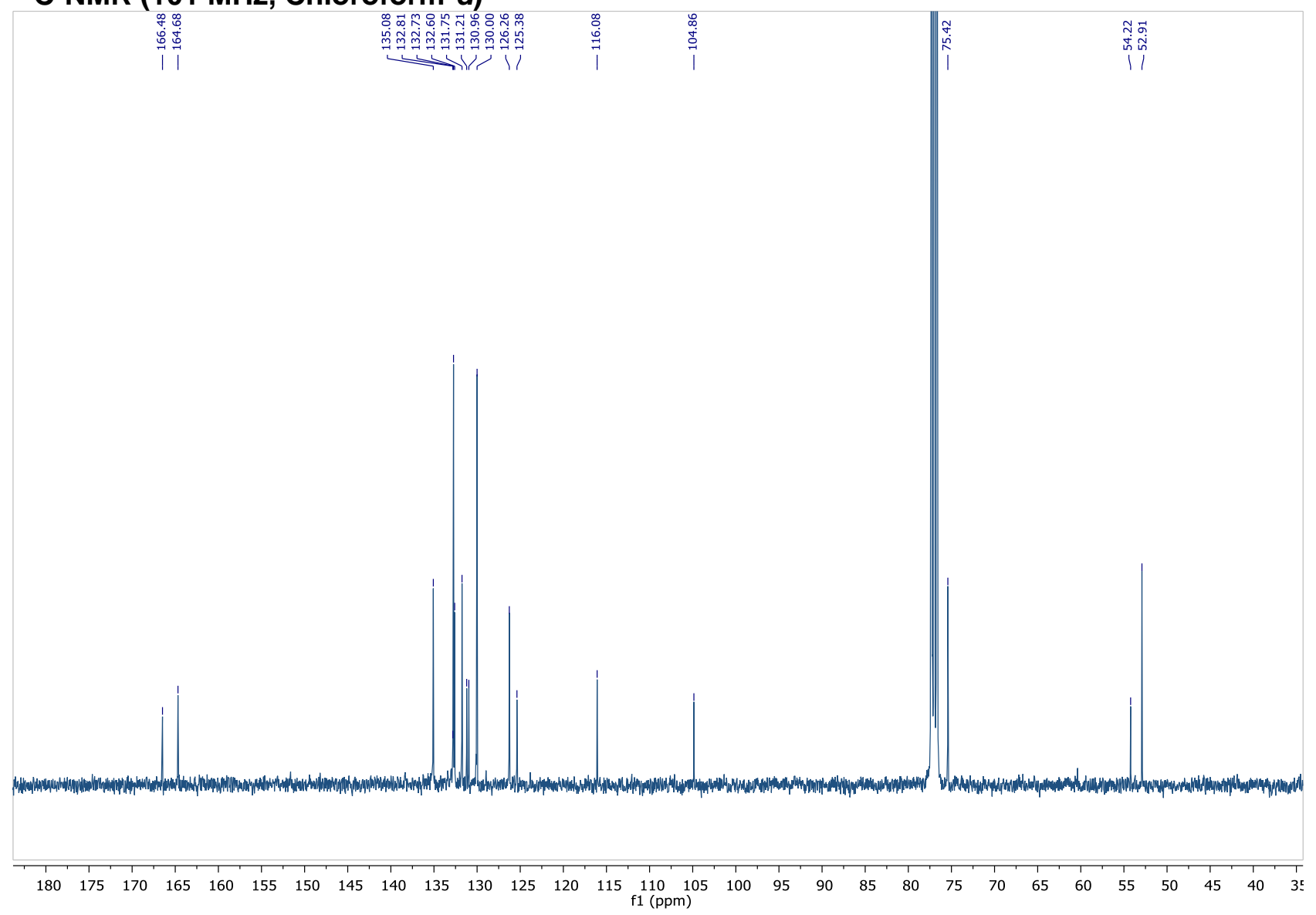

74 
${ }^{1} \mathrm{H}-\mathrm{NMR}$ (400 MHz, Chloroform- $d$ )

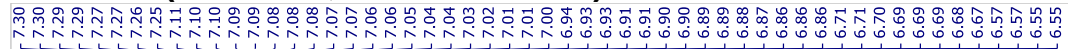

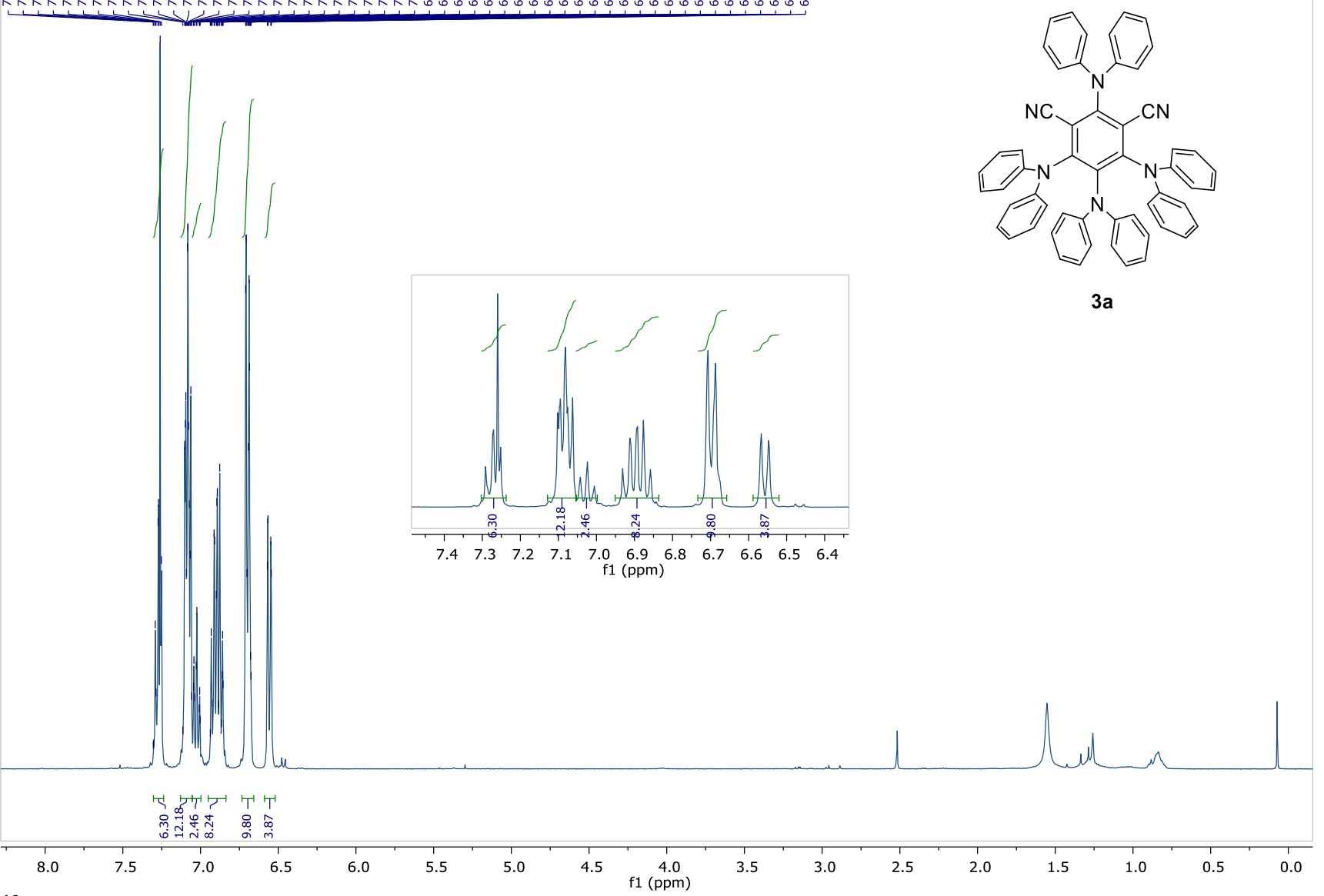

${ }^{13} \mathrm{C}-N M R$ (101 MHz, Chloroform- $d$ )

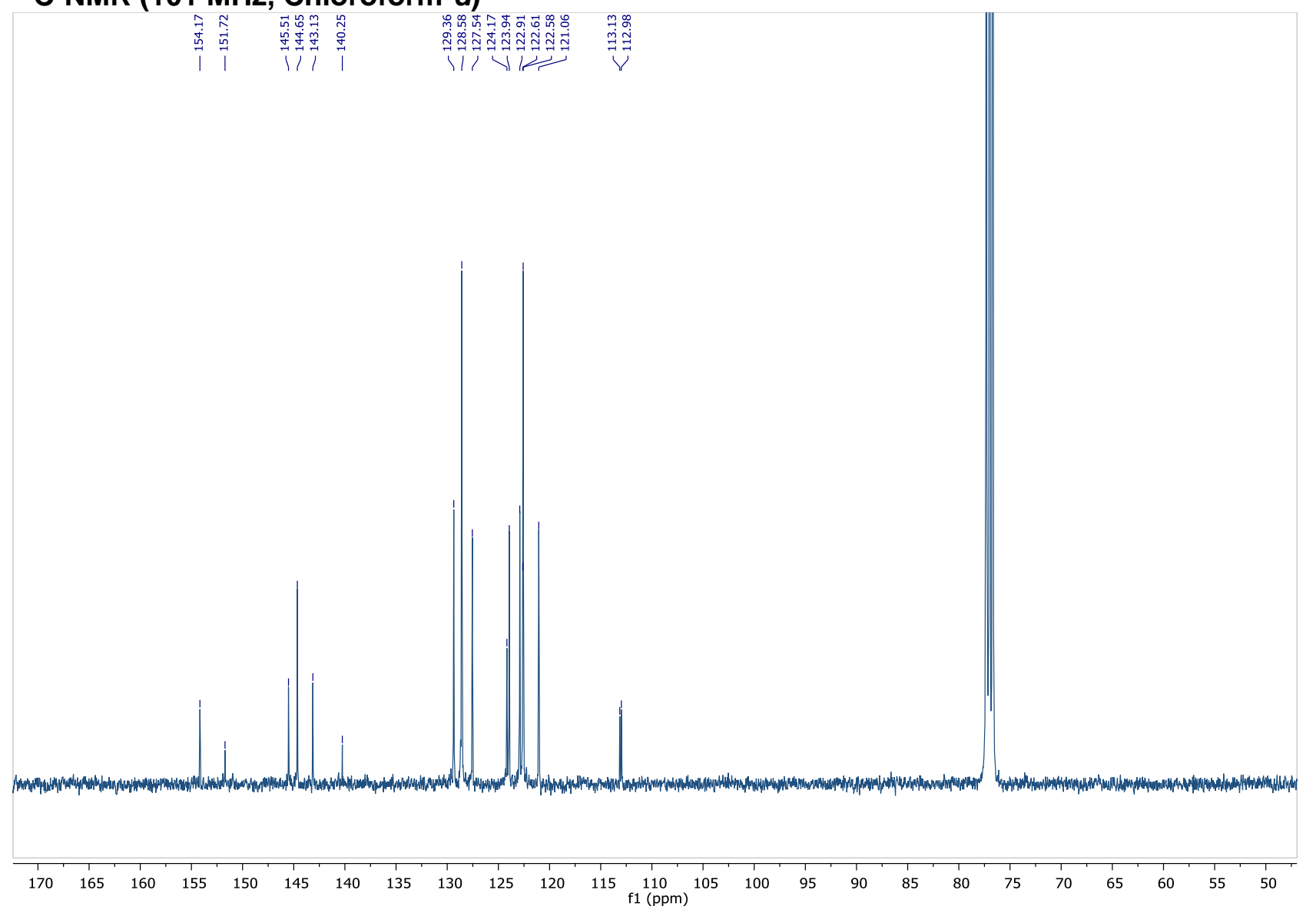


${ }^{1} \mathrm{H}-\mathrm{NMR}$ (400 MHz, Chloroform-d)

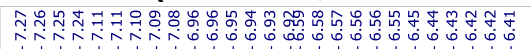

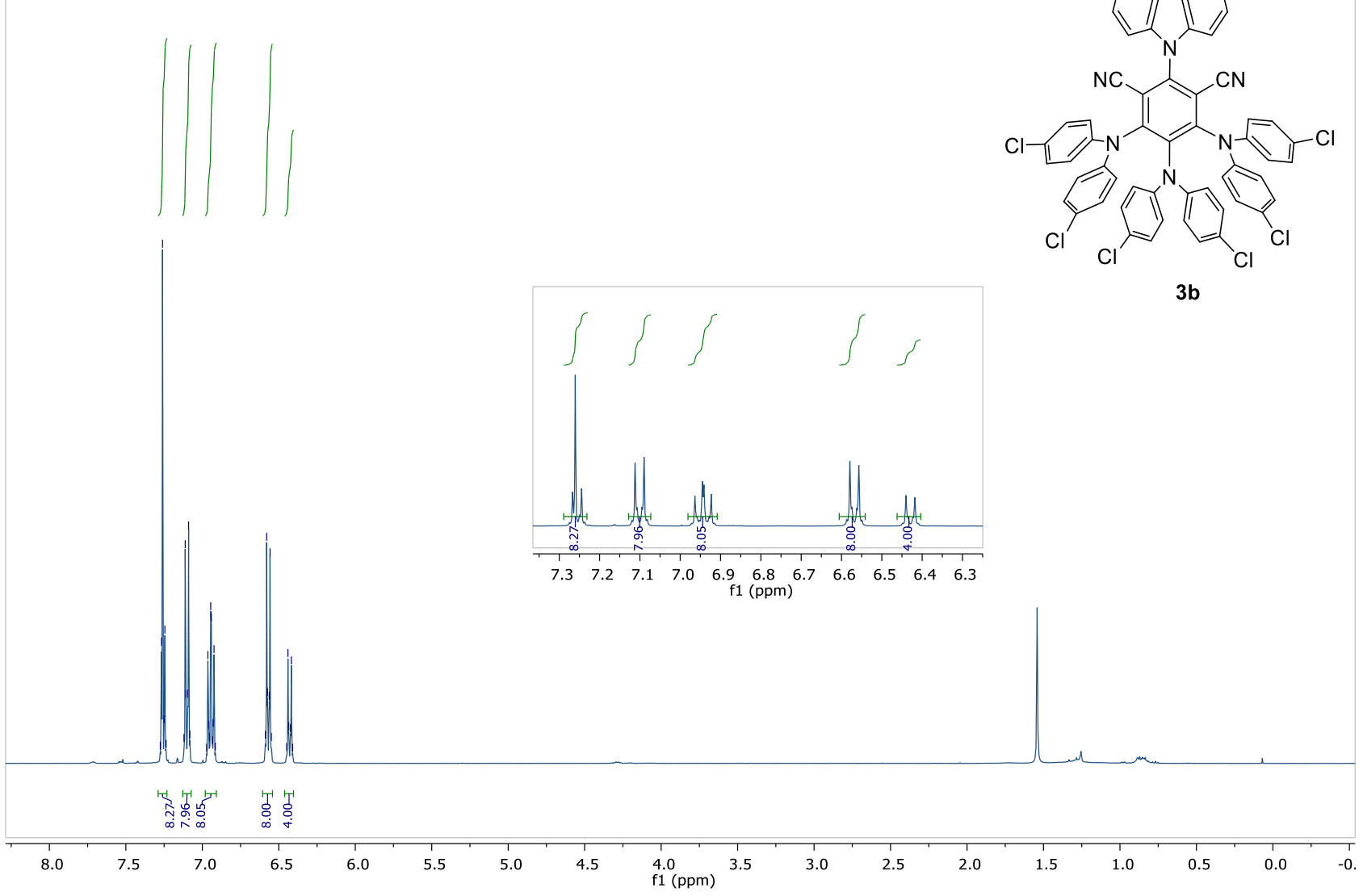

${ }^{13} \mathrm{C}-\mathrm{NMR}$ (101 MHz, Chloroform- $d$ )

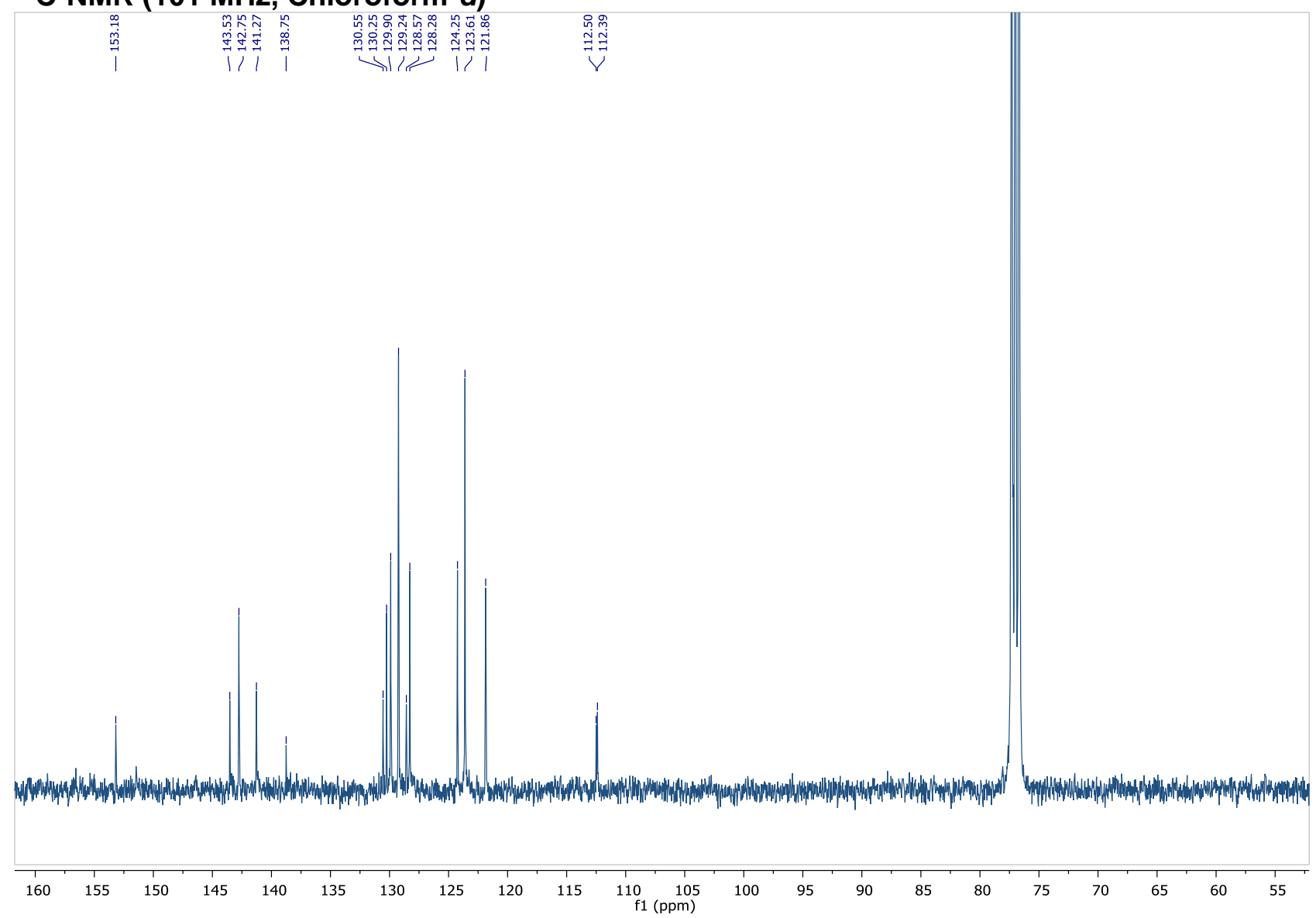


${ }^{1} \mathrm{H}-\mathrm{NMR}$ (400 MHz, Chloroform- $d$ )

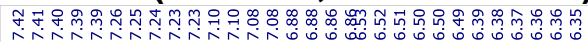

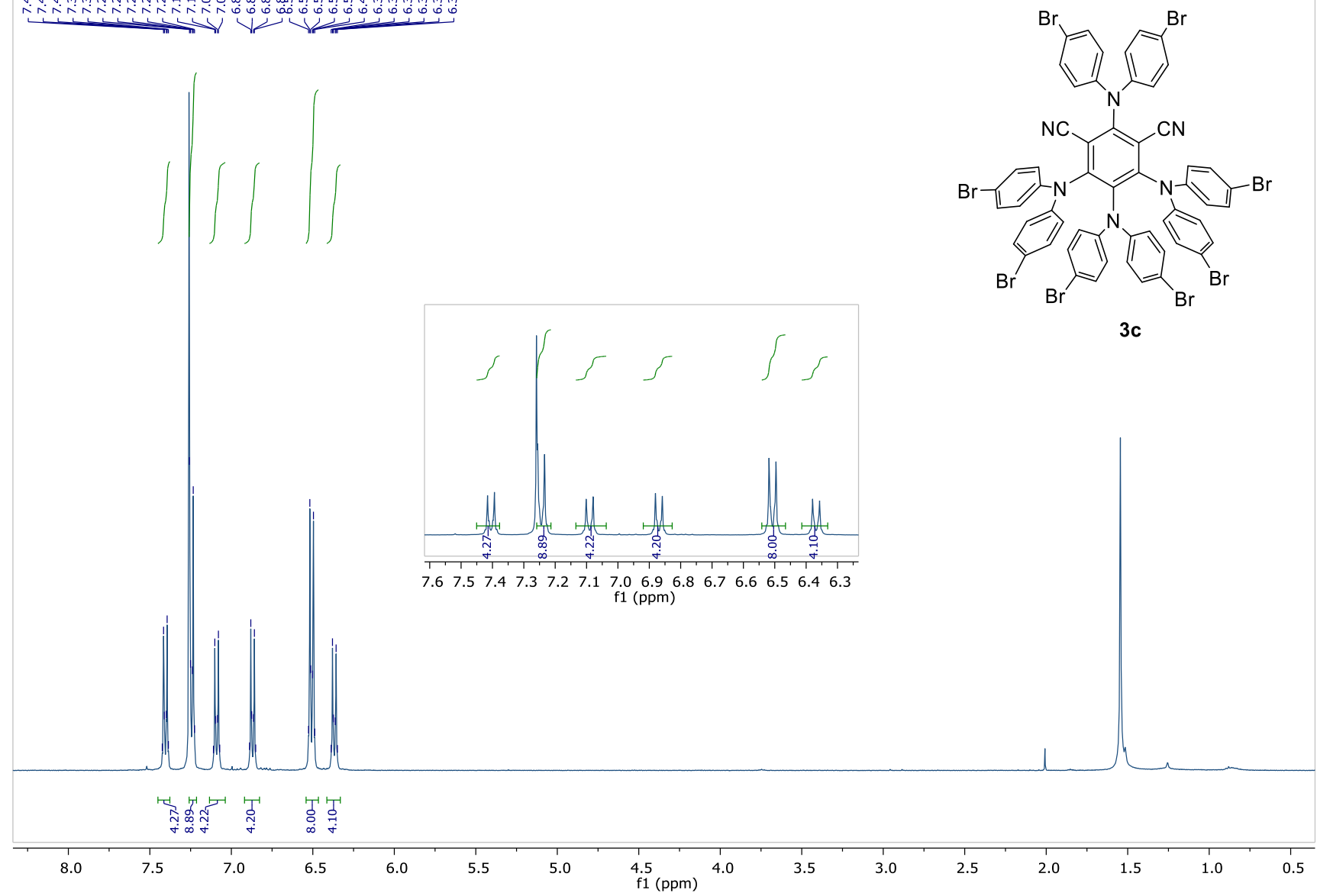

${ }^{13}$ C-NMR (101 MHz, Chloroform- $d$ )

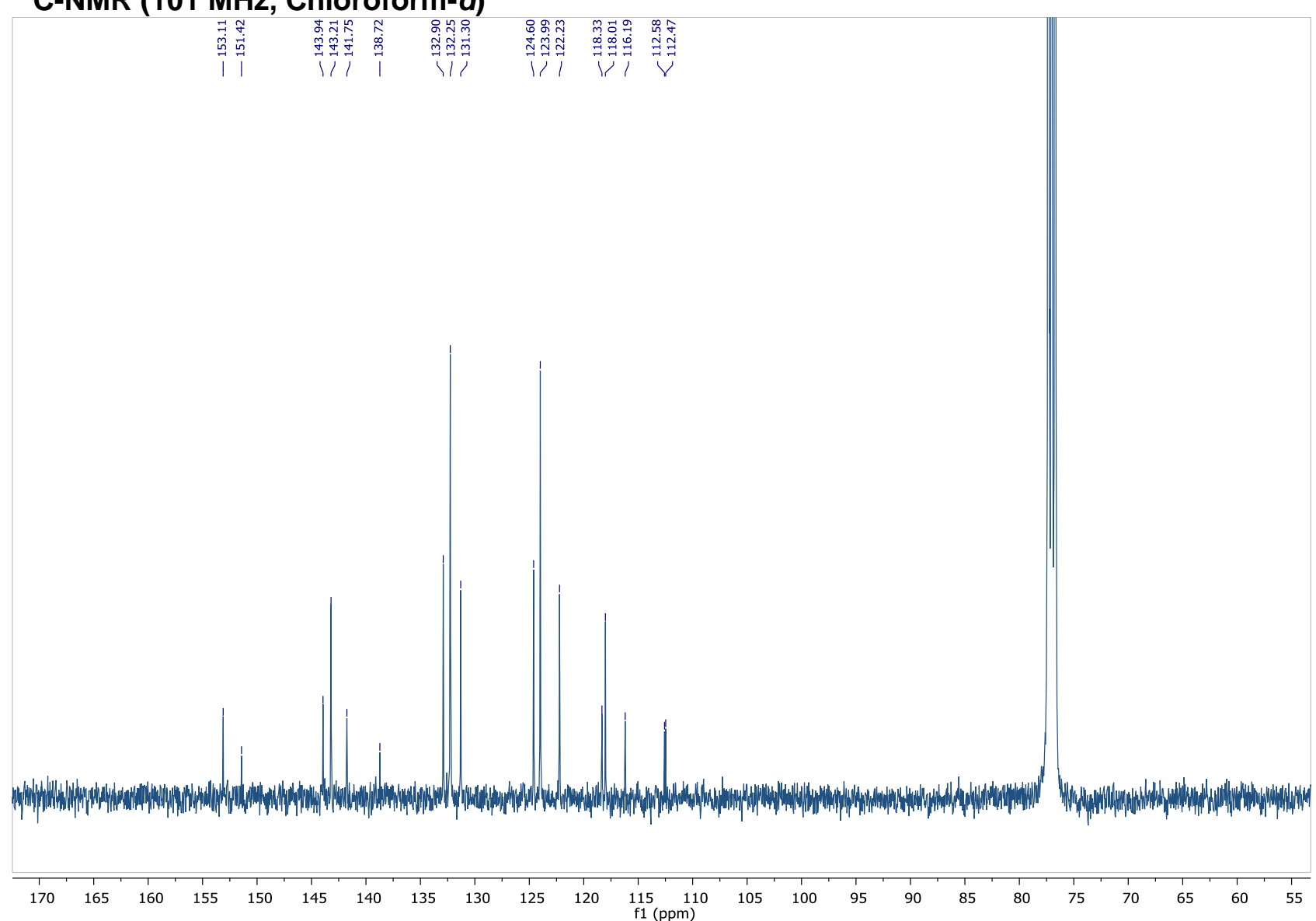


${ }^{1} \mathrm{H}-\mathrm{NMR}(400 \mathrm{MHz}$, Chloroform- $d)$

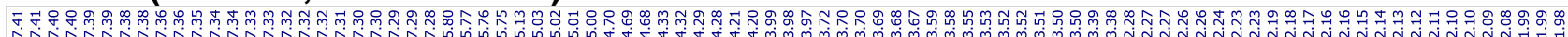

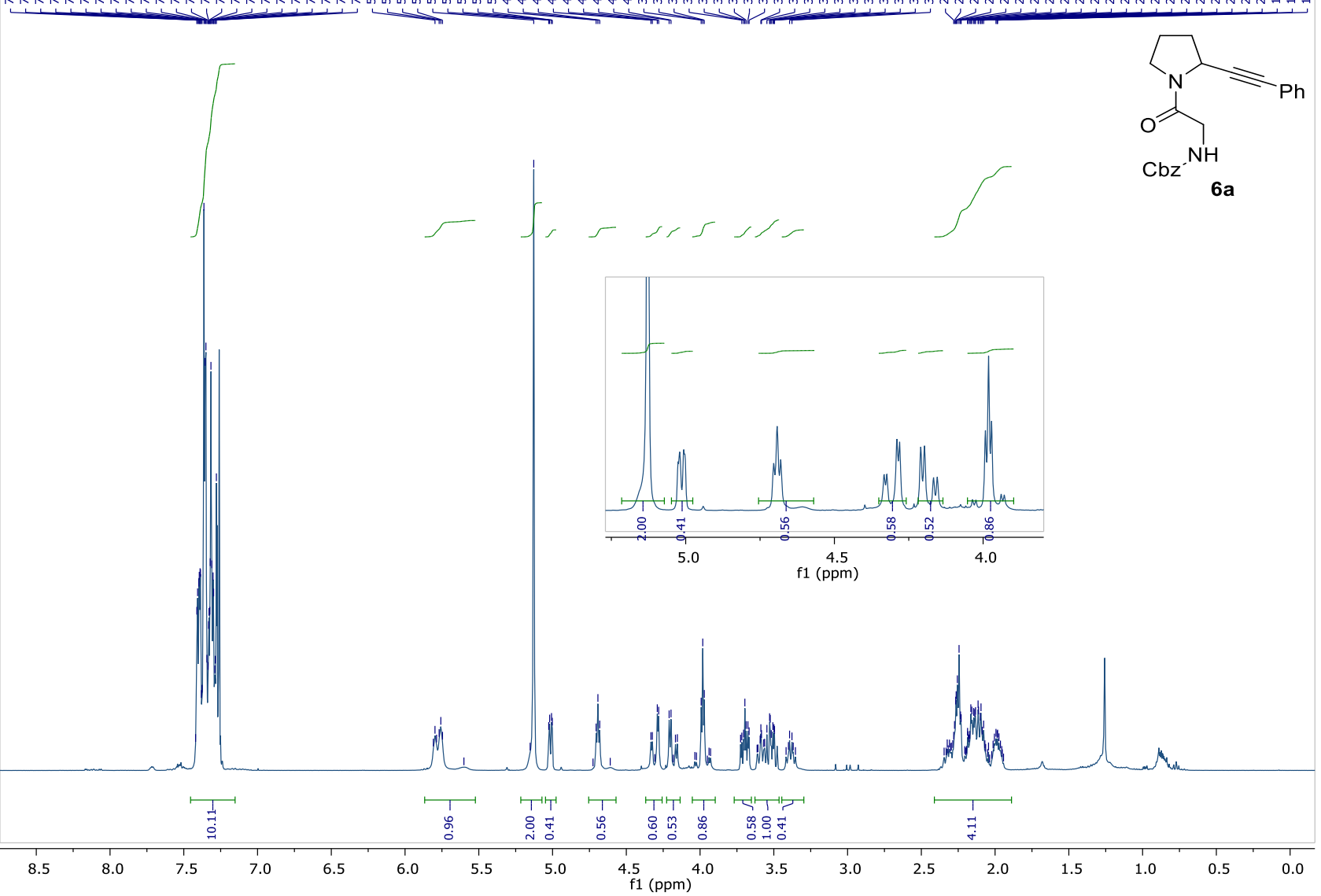

${ }^{13} \mathrm{C}-\mathrm{NMR}$ (101 MHz, Chloroform- $d$ )

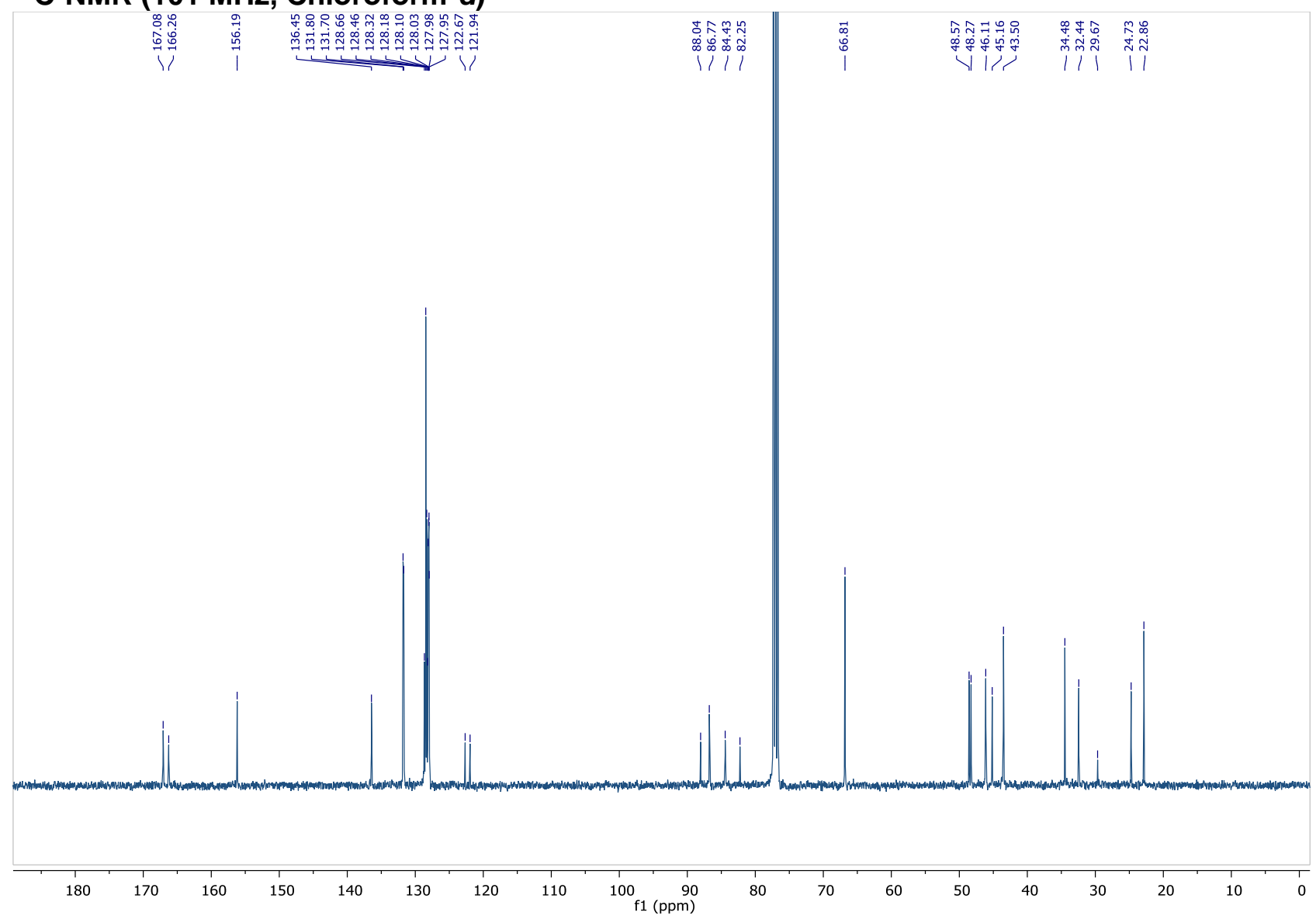


cosy (Chloroform- $d$ )

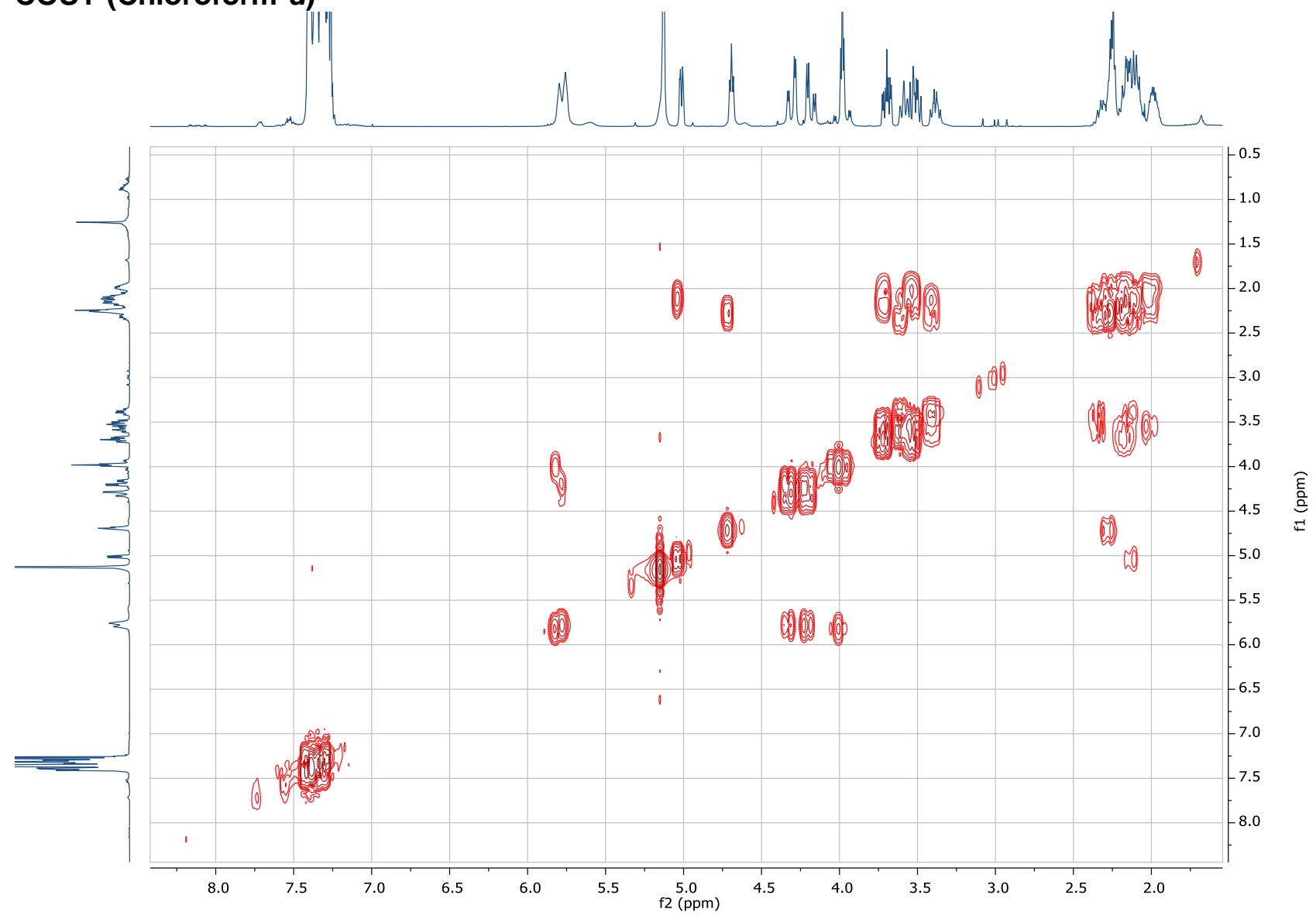

HSQC (Chloroform- $d$ )

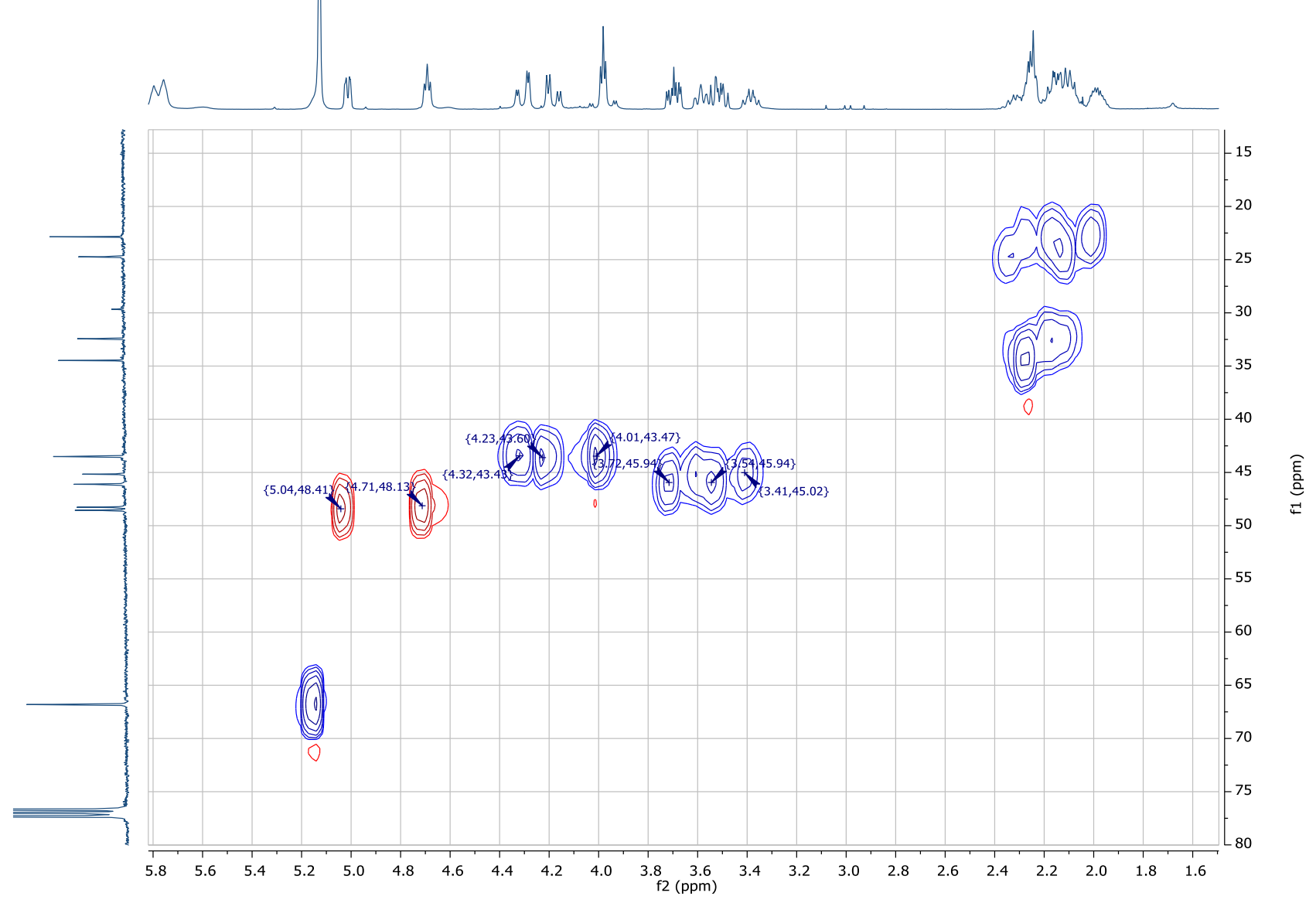




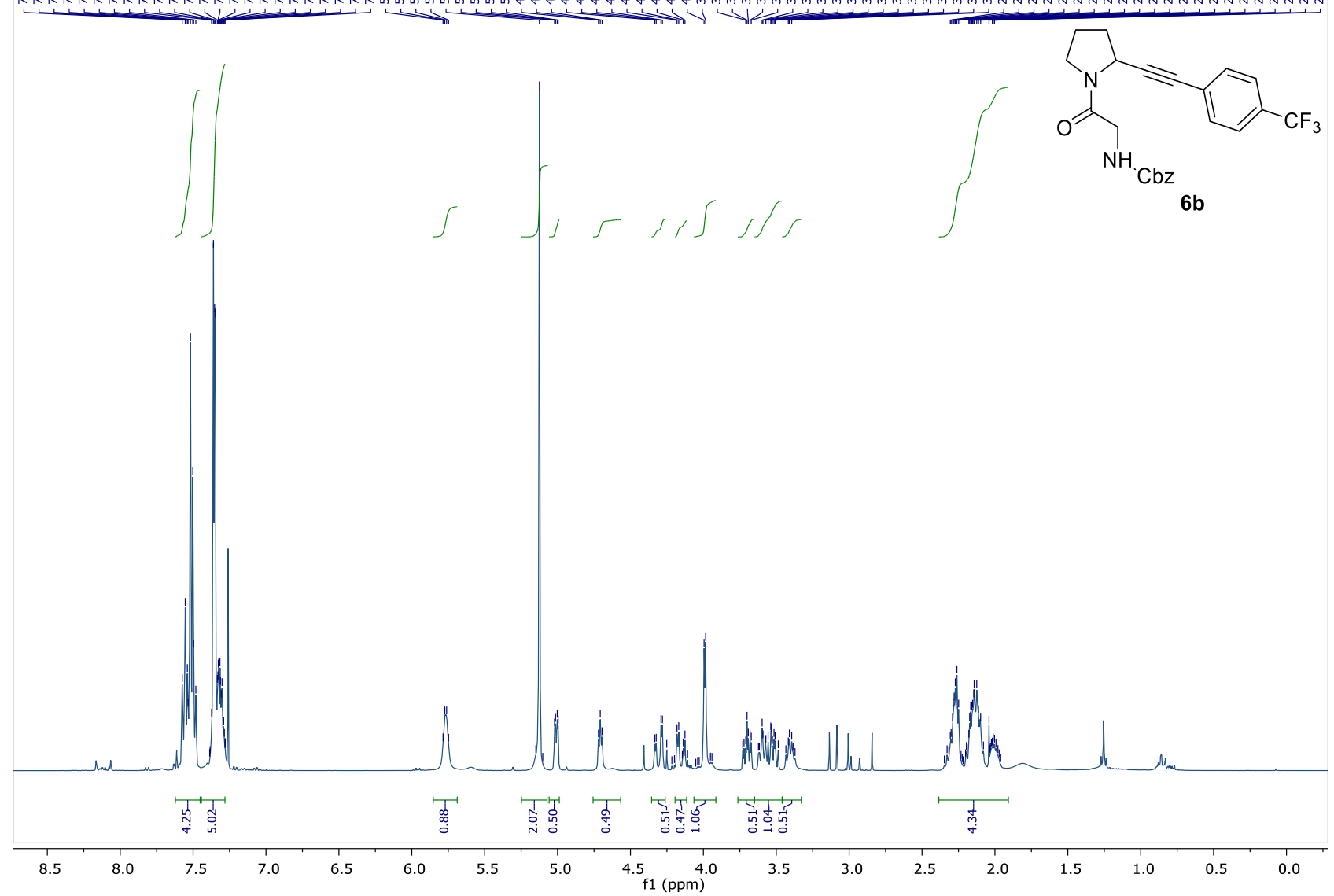

${ }^{13} \mathrm{C}-\mathrm{NMR}(101 \mathrm{MHz}$, Chloroform- $d)$

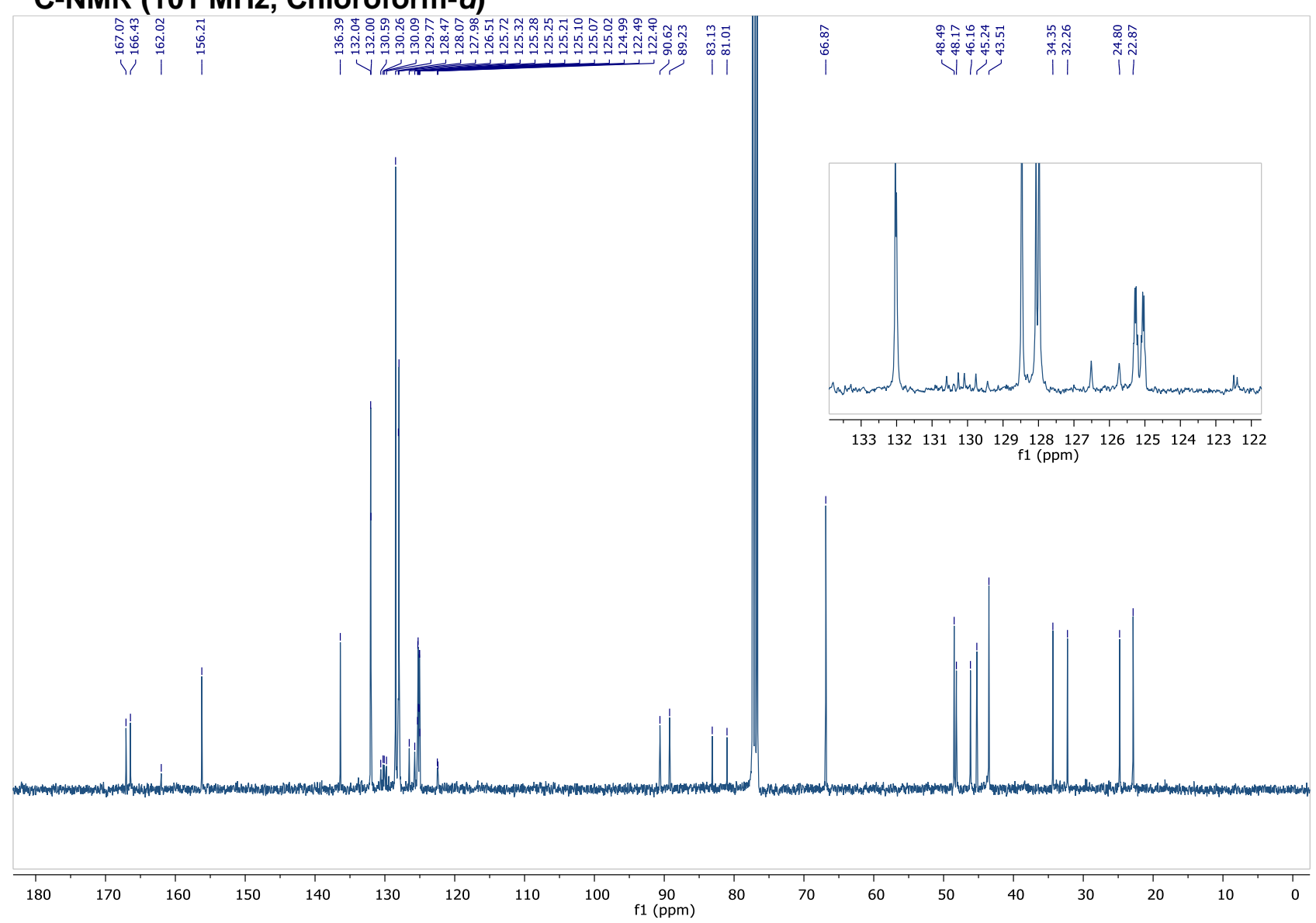


${ }^{19}$ F NMR (376 MHz, Chloroform-d)

ชู

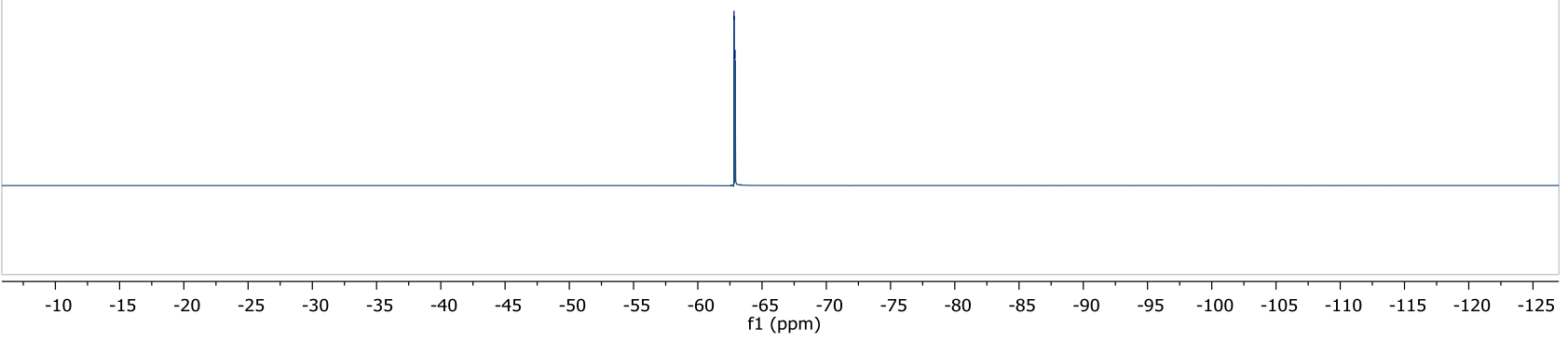


${ }^{1} \mathrm{H}-\mathrm{NMR}(400 \mathrm{MHz}$, Chloroform- $d)$

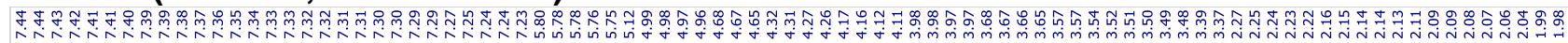

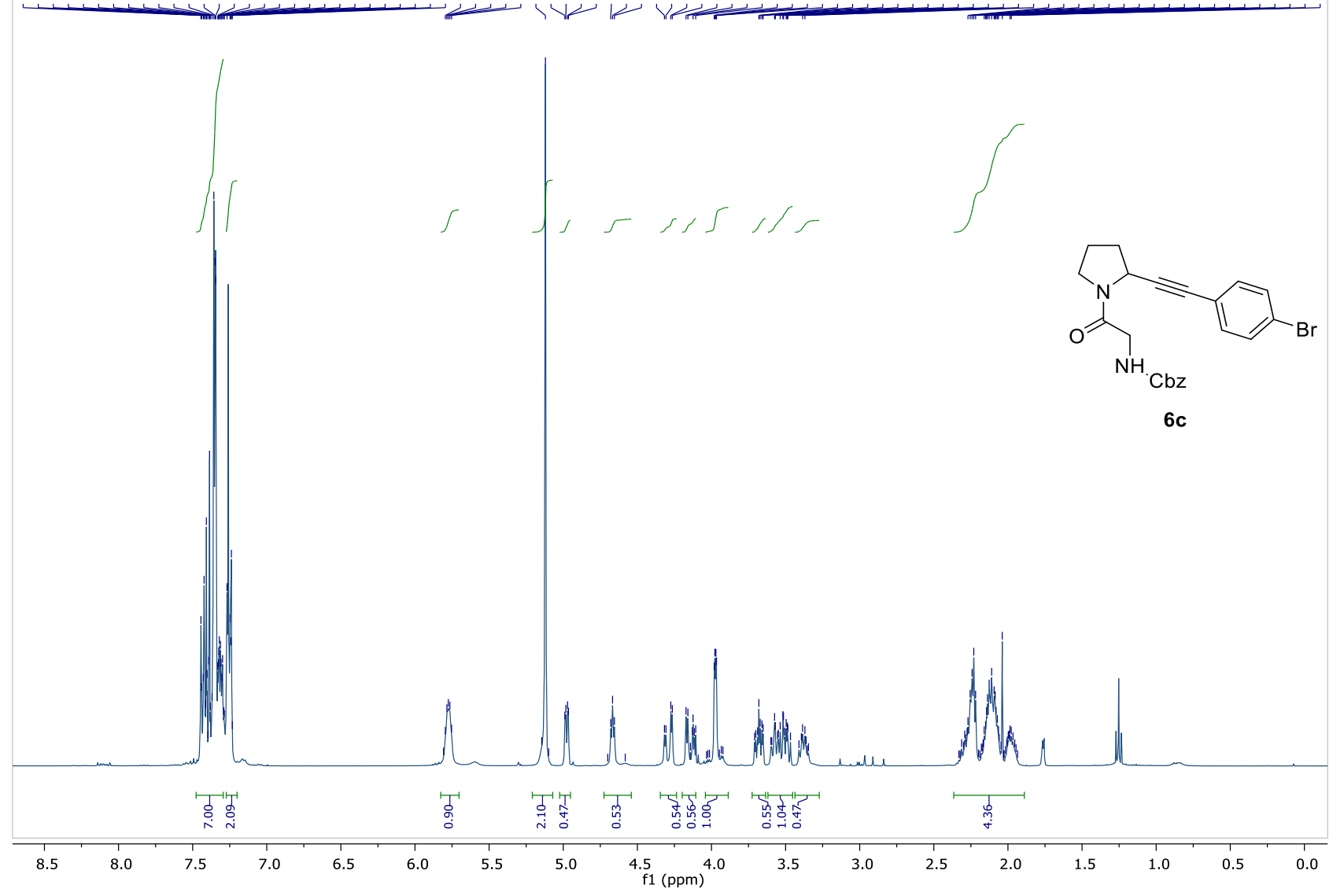

${ }^{13} \mathrm{C}-\mathrm{NMR}(101 \mathrm{MHz}$, Chloroform- $d)$

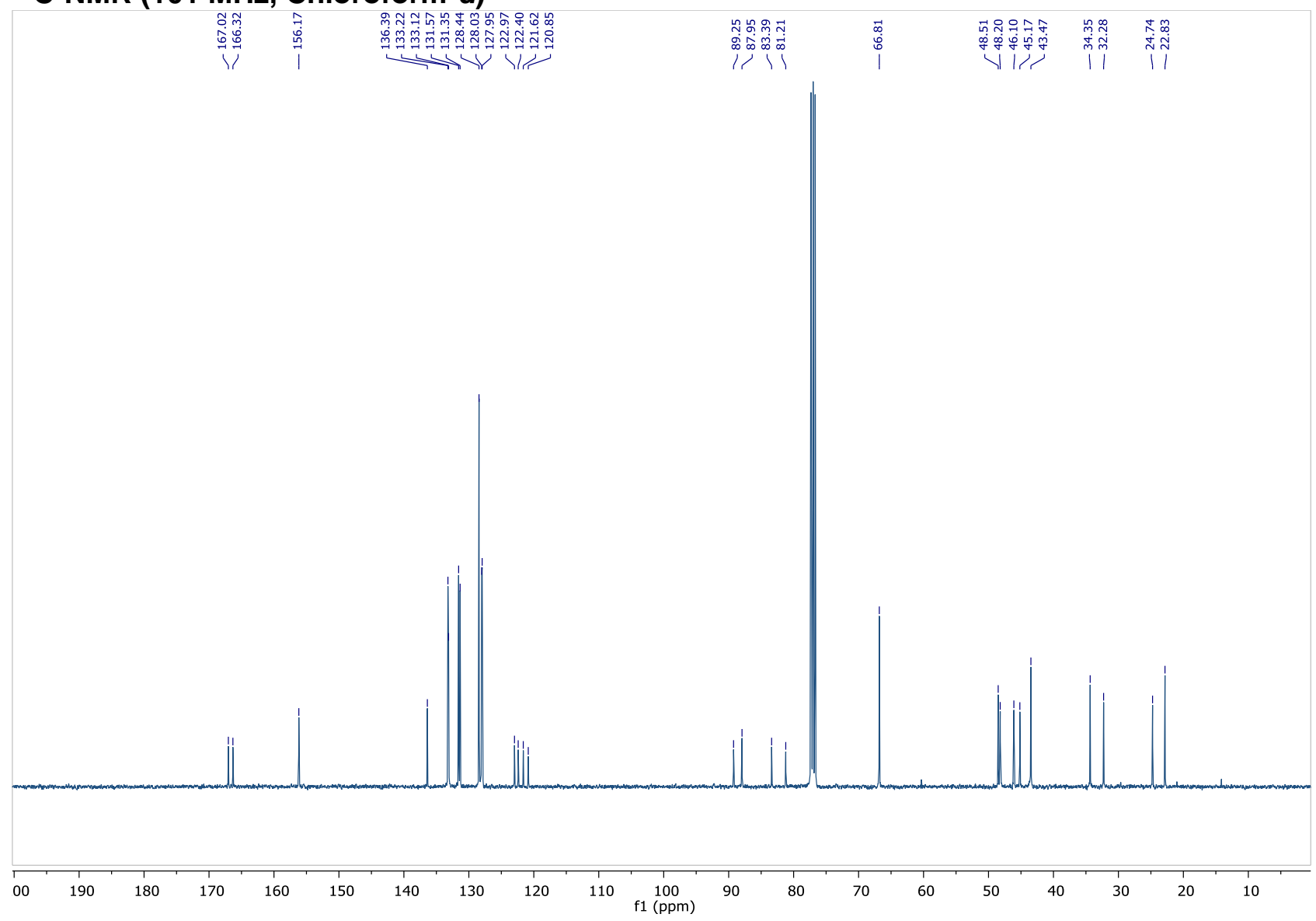


${ }^{1} \mathrm{H}-\mathrm{NMR}(400 \mathrm{MHz}$, Chloroform- $d)$

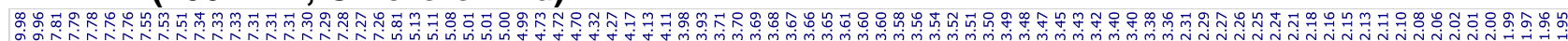

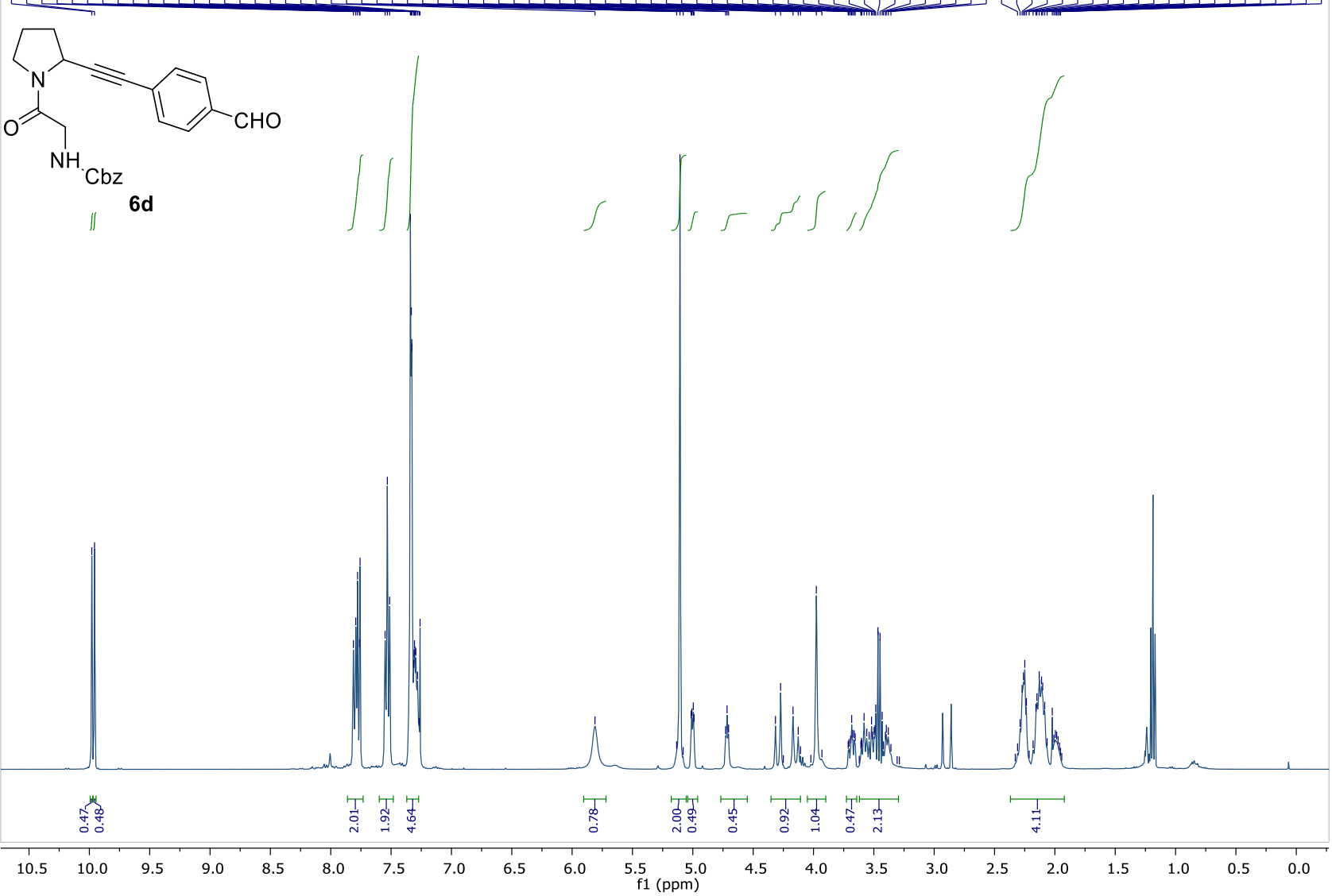

${ }^{13} \mathrm{C}-\mathrm{NMR}(101 \mathrm{MHz}$, Chloroform-d)

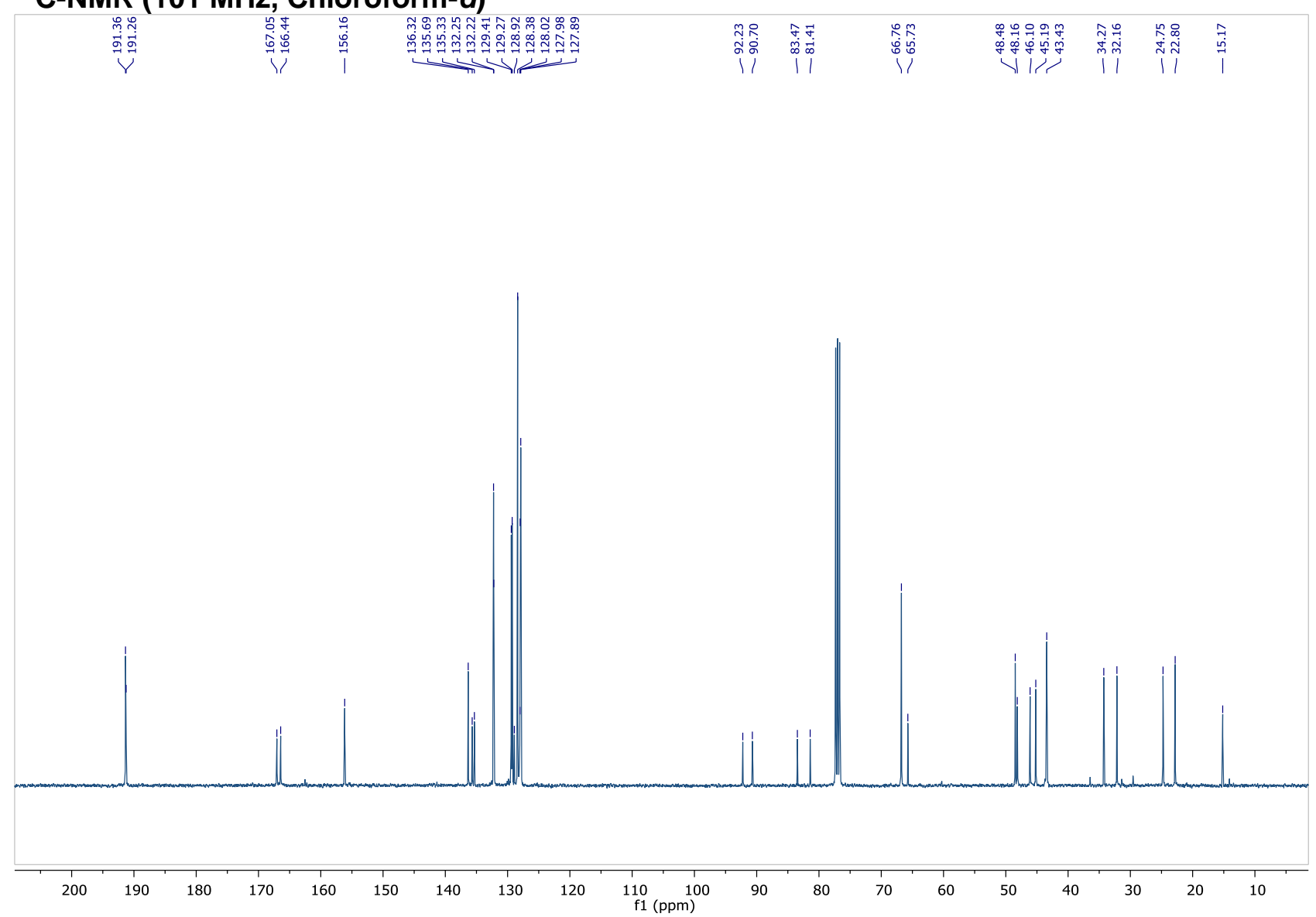


${ }^{1} \mathrm{H}-\mathrm{NMR}$ (400 MHz, Chloroform- $d$ )

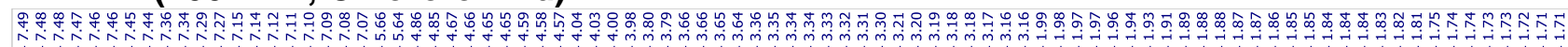

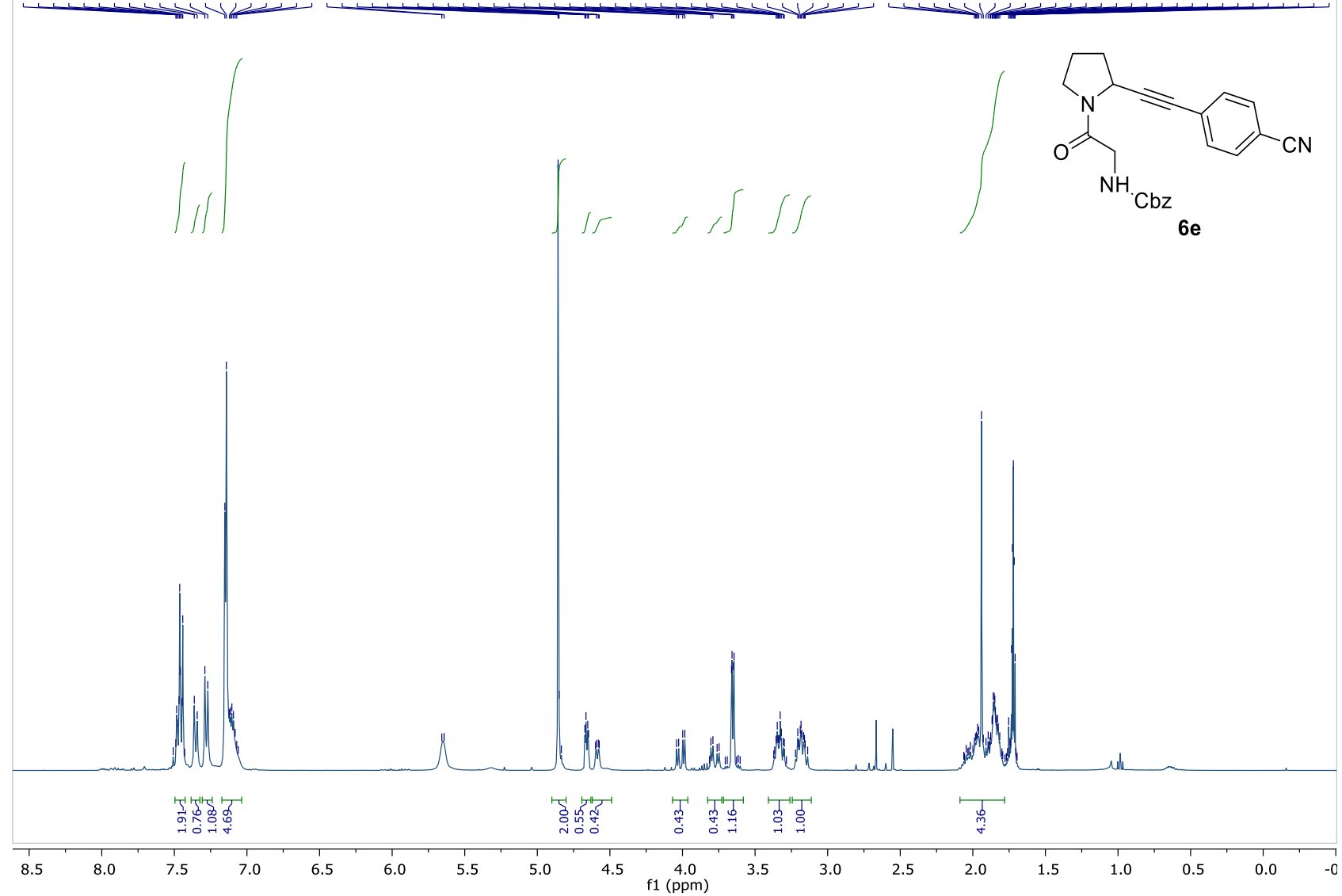

${ }^{13} \mathrm{C}-\mathrm{NMR}$ (101 MHz, Chloroform-d)

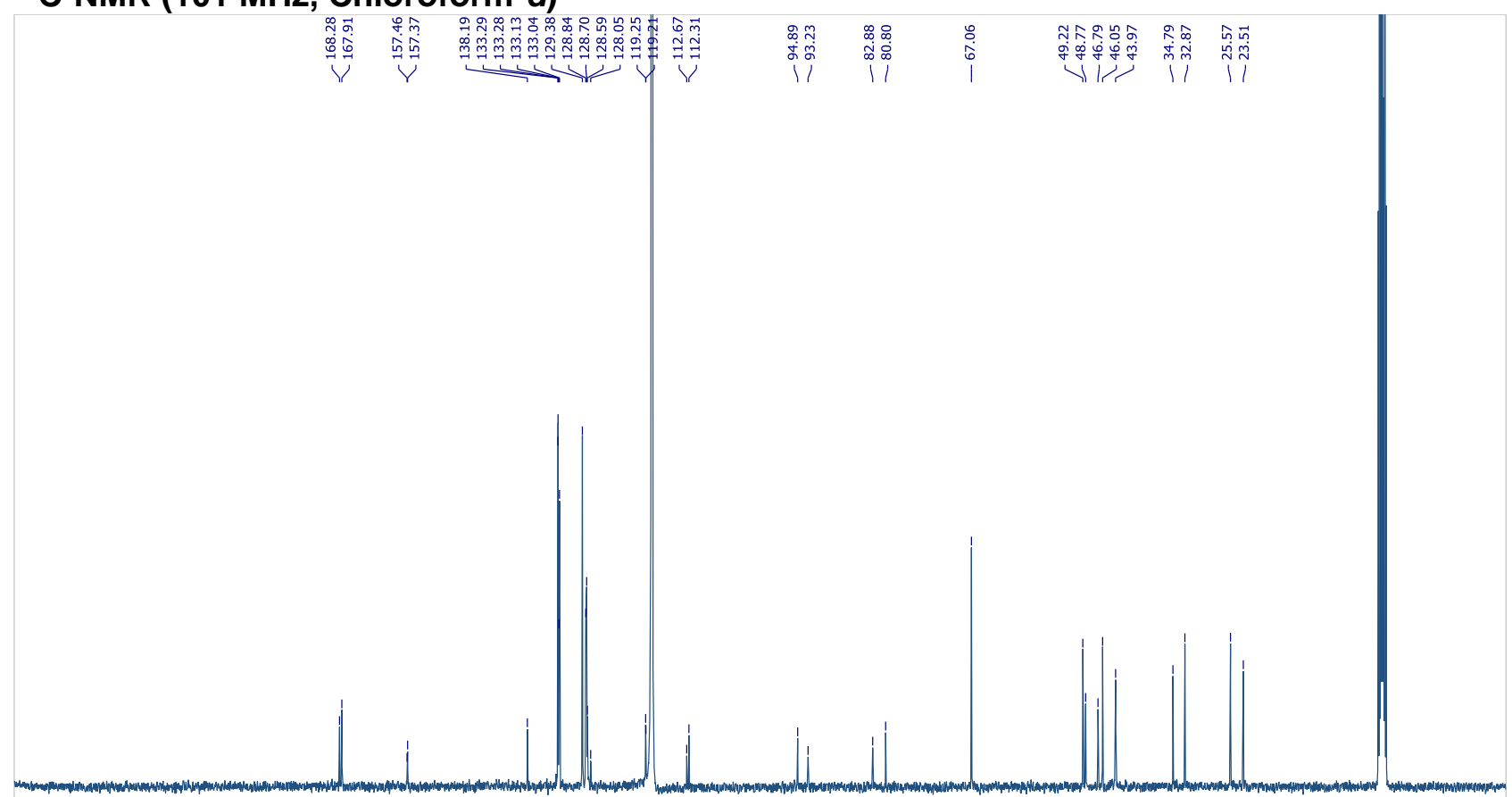

20

$0210 \quad 200$ $\begin{array}{lllllllllll}1 & 190 & 180 & 170 & 160 & 150 & 140 & 130 & 120 & 110 & 100 \\ \mathrm{f} 1(\mathrm{ppm})\end{array}$ 


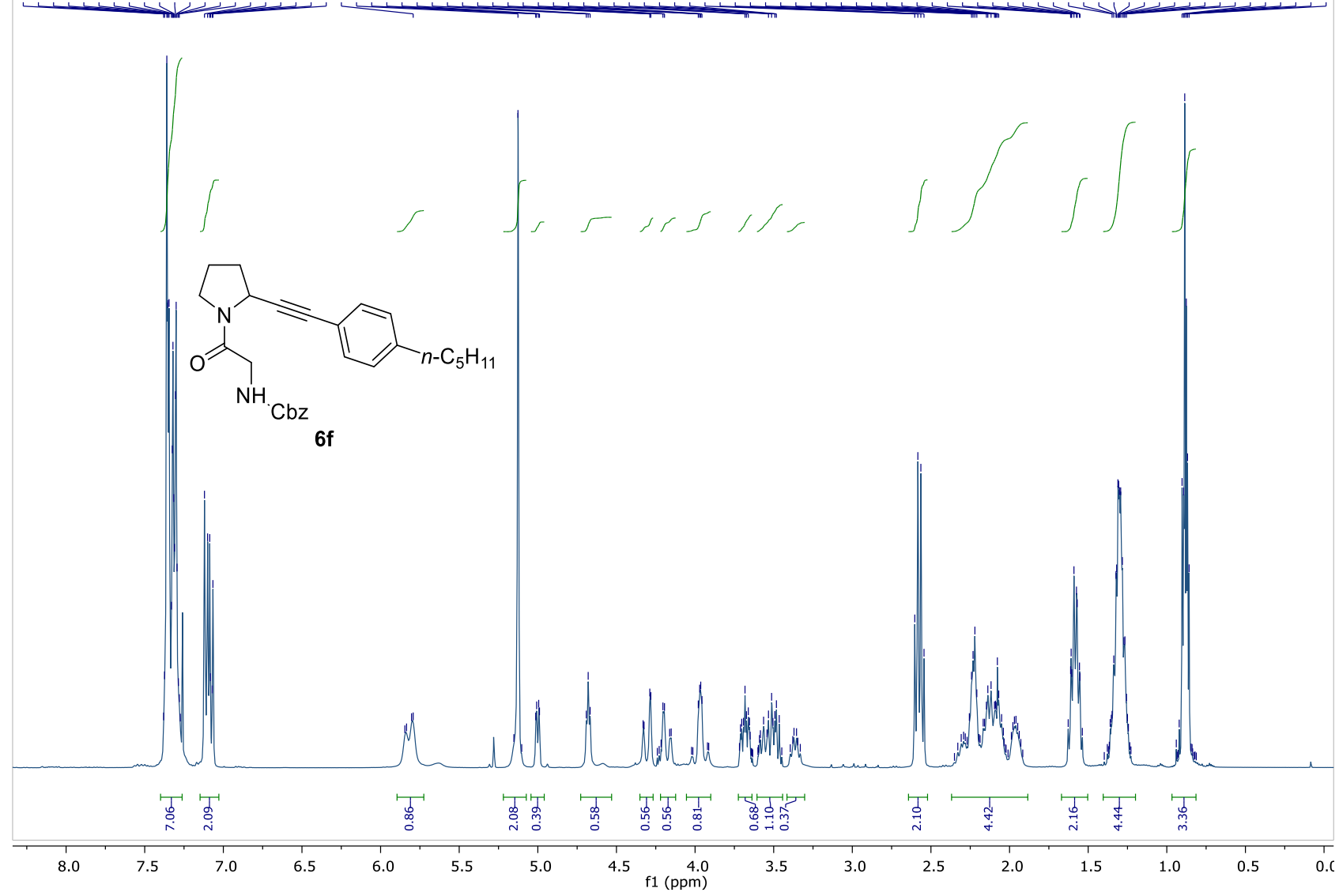

${ }^{13} \mathrm{C}-\mathrm{NMR}$ (101 MHz, Chloroform- $\left.d\right)$

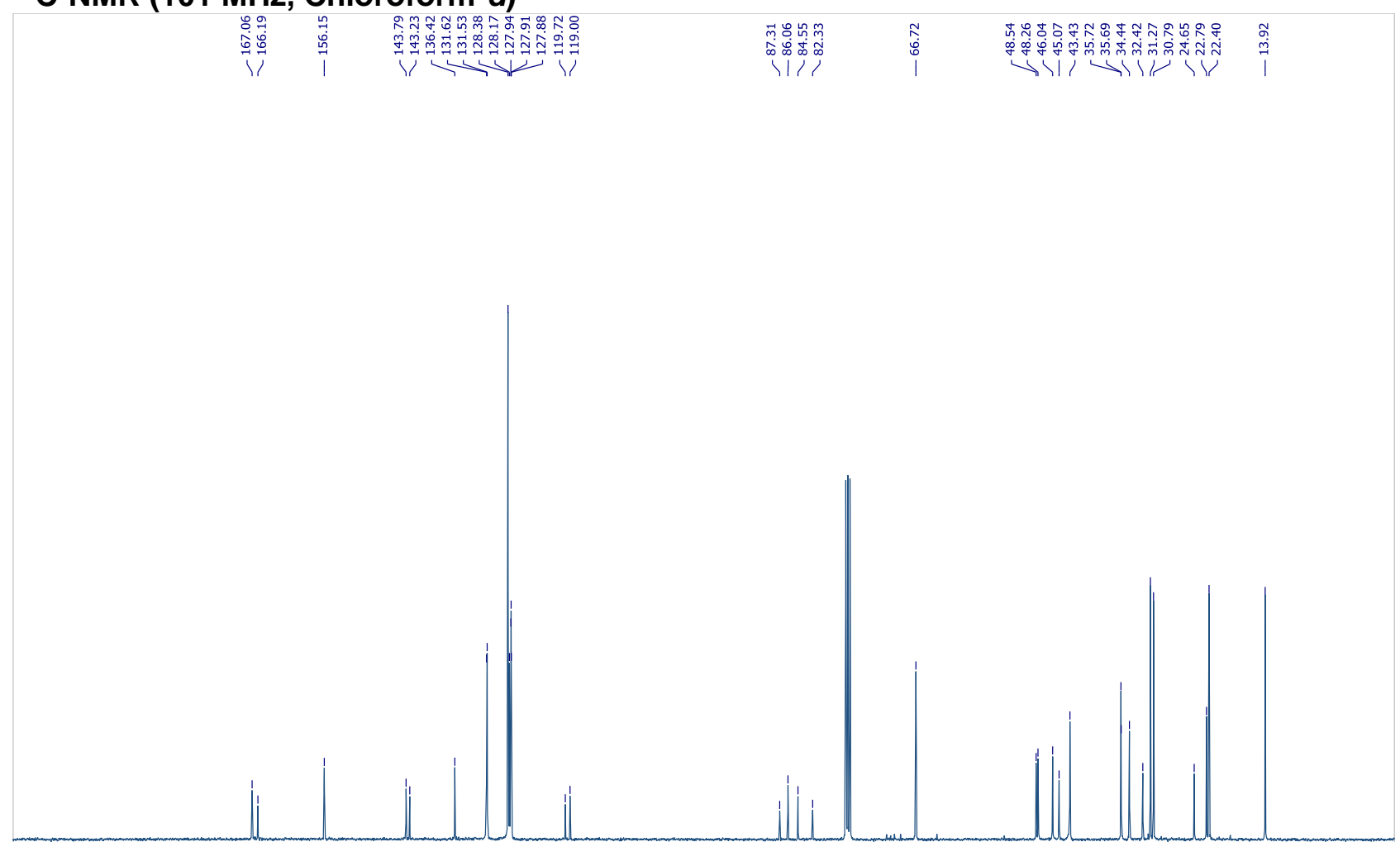


${ }^{1} \mathrm{H}-\mathrm{NMR}$ (400 MHz, Chloroform-d)

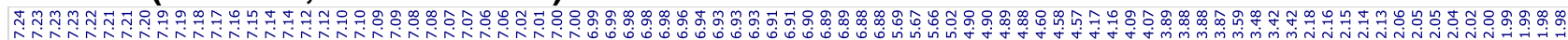

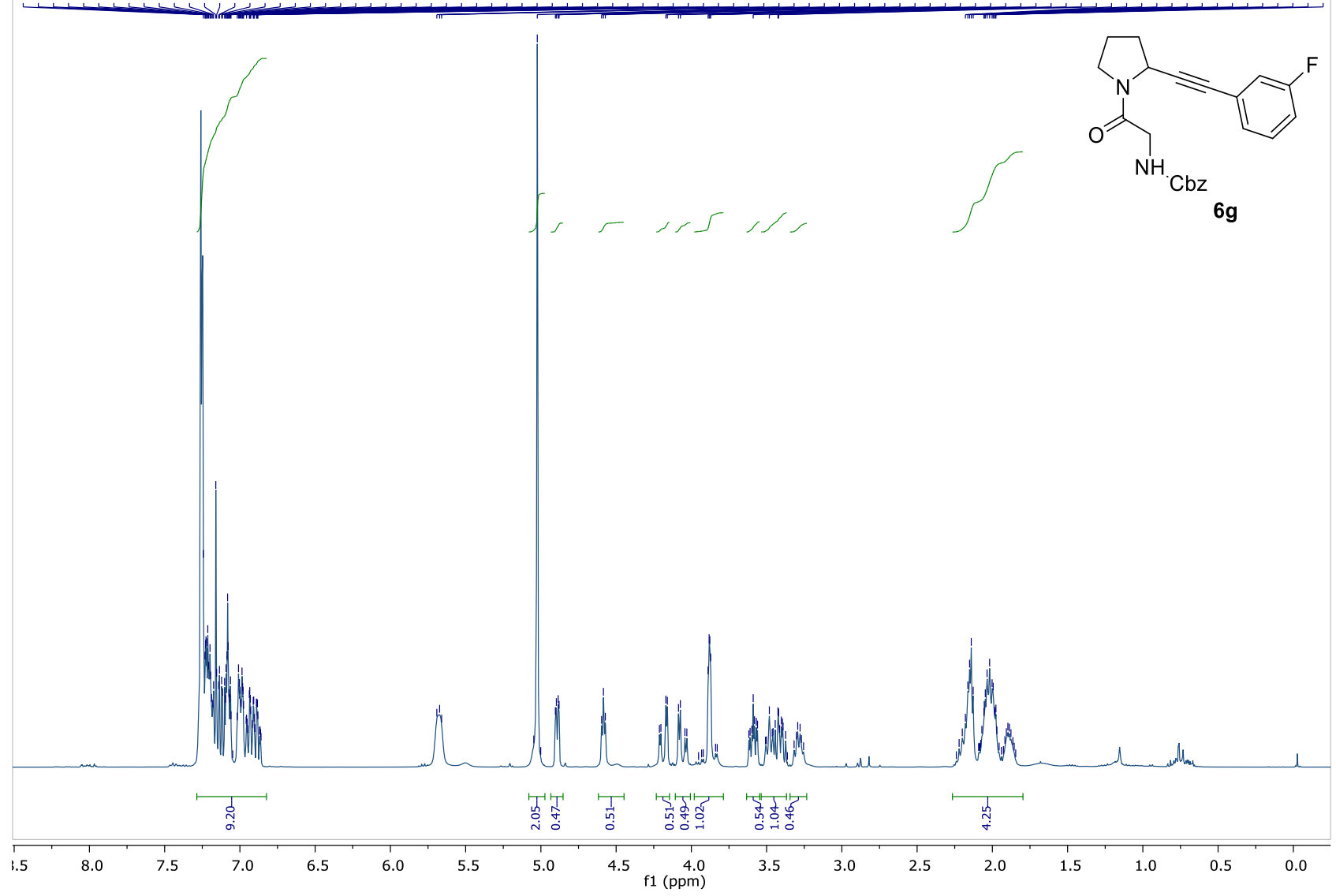

${ }^{13} \mathrm{C}-\mathrm{NMR}(101 \mathrm{MHz}$, Chloroform- $d)$

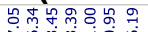

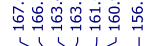

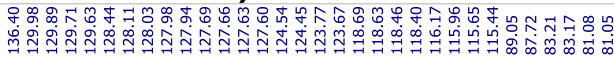

Y I

s.

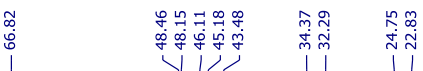

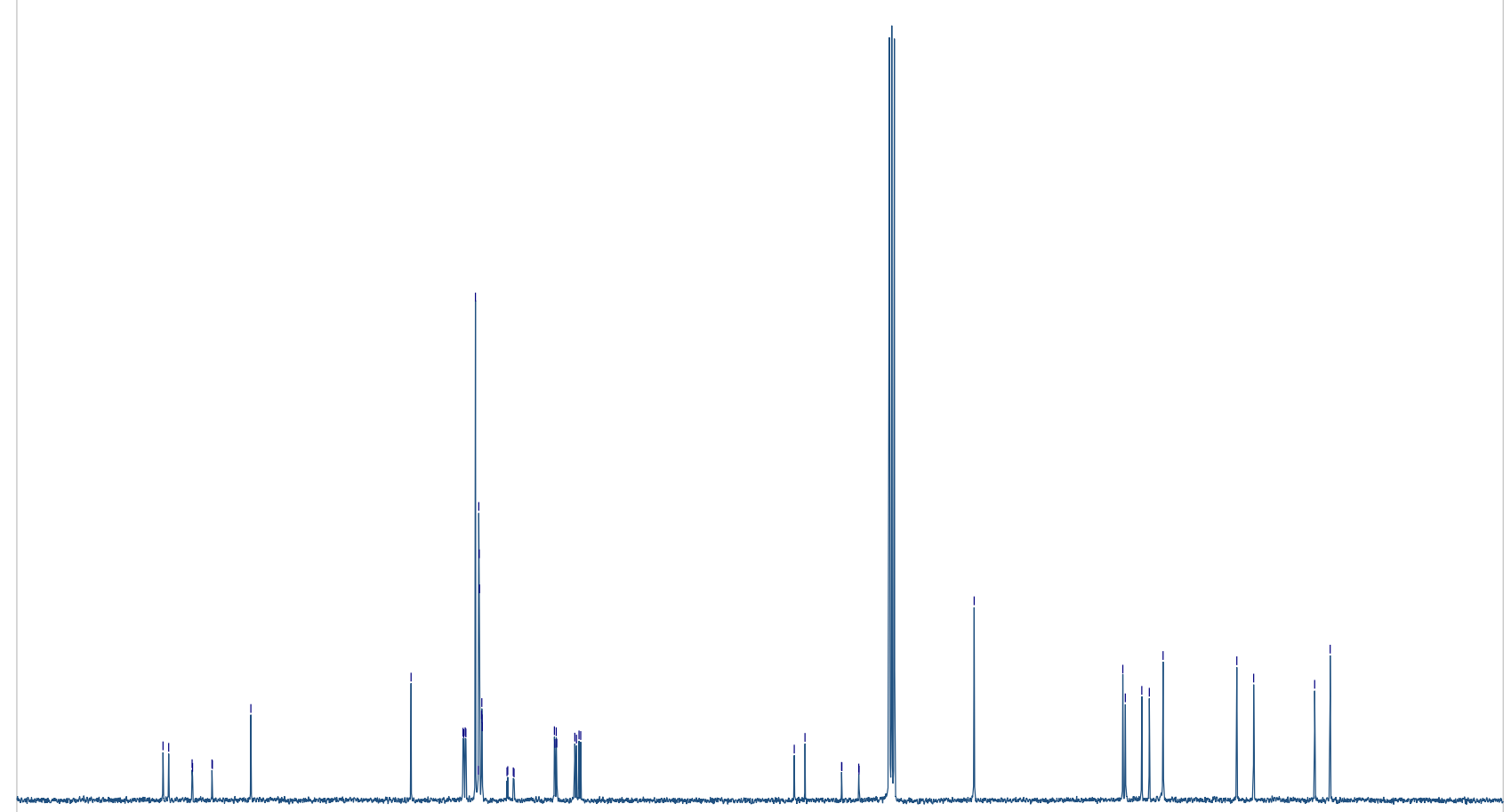

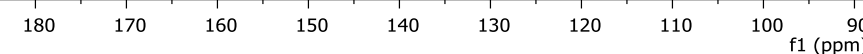


${ }^{19}$ F NMR (376 MHz, Chloroform-d)

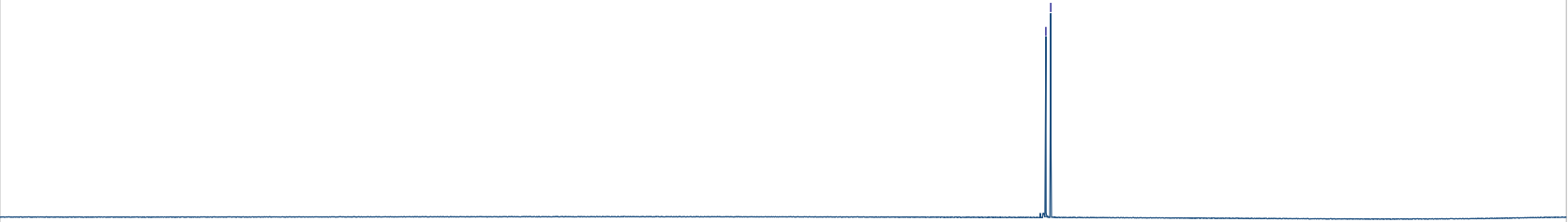

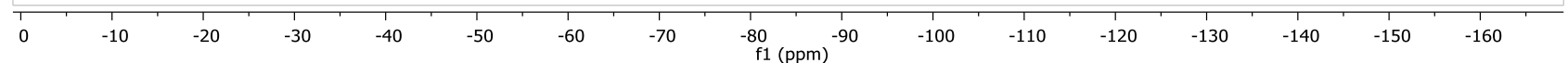


${ }^{1} \mathrm{H}-\mathrm{NMR}(400 \mathrm{MHz}$, Chloroform- $d)$

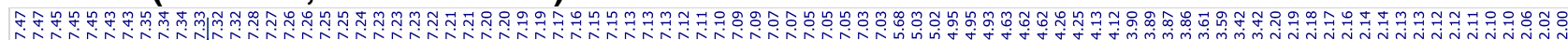

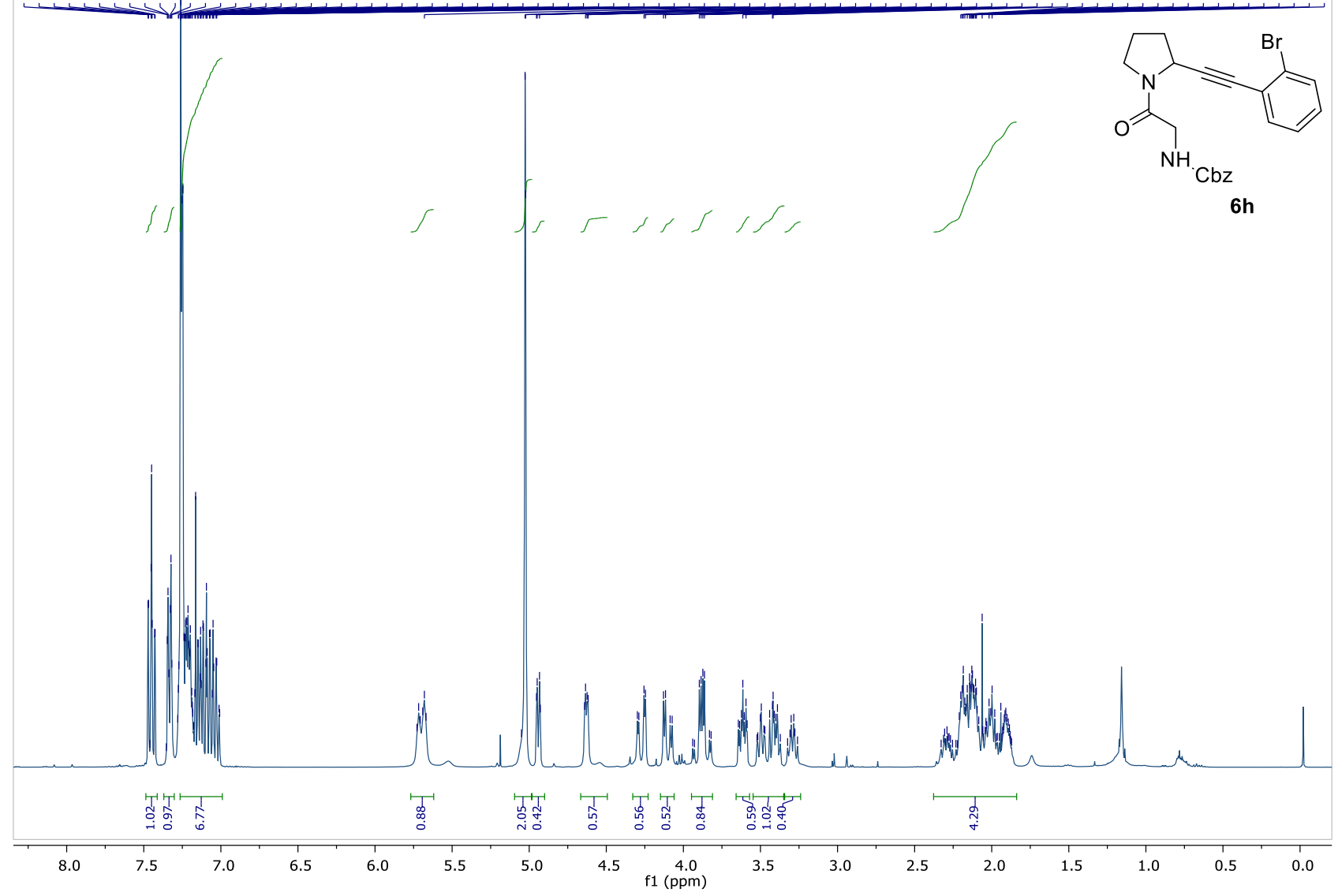

${ }^{13} \mathrm{C}-\mathrm{NMR}(101 \mathrm{MHz}$, Chloroform-d)

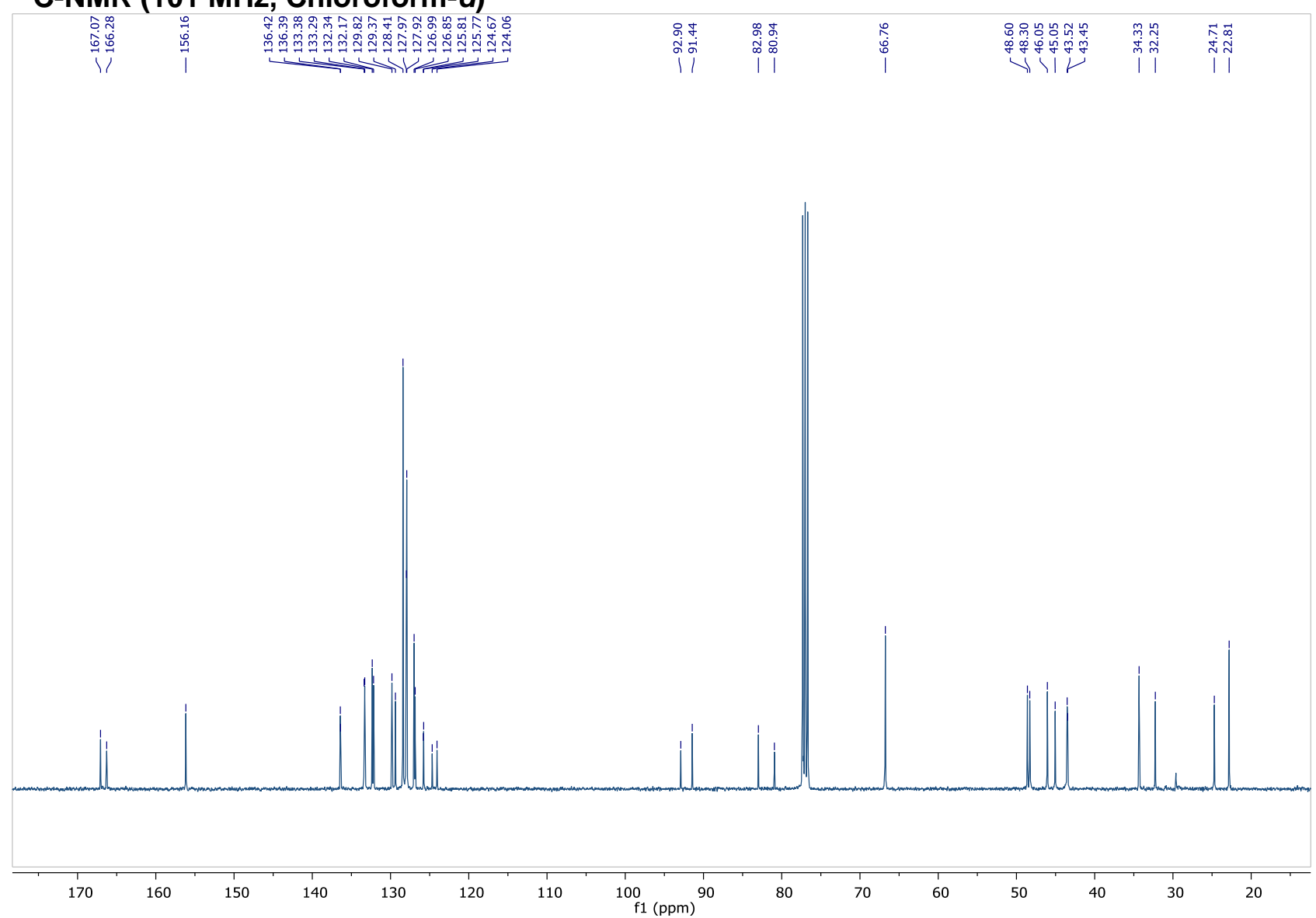




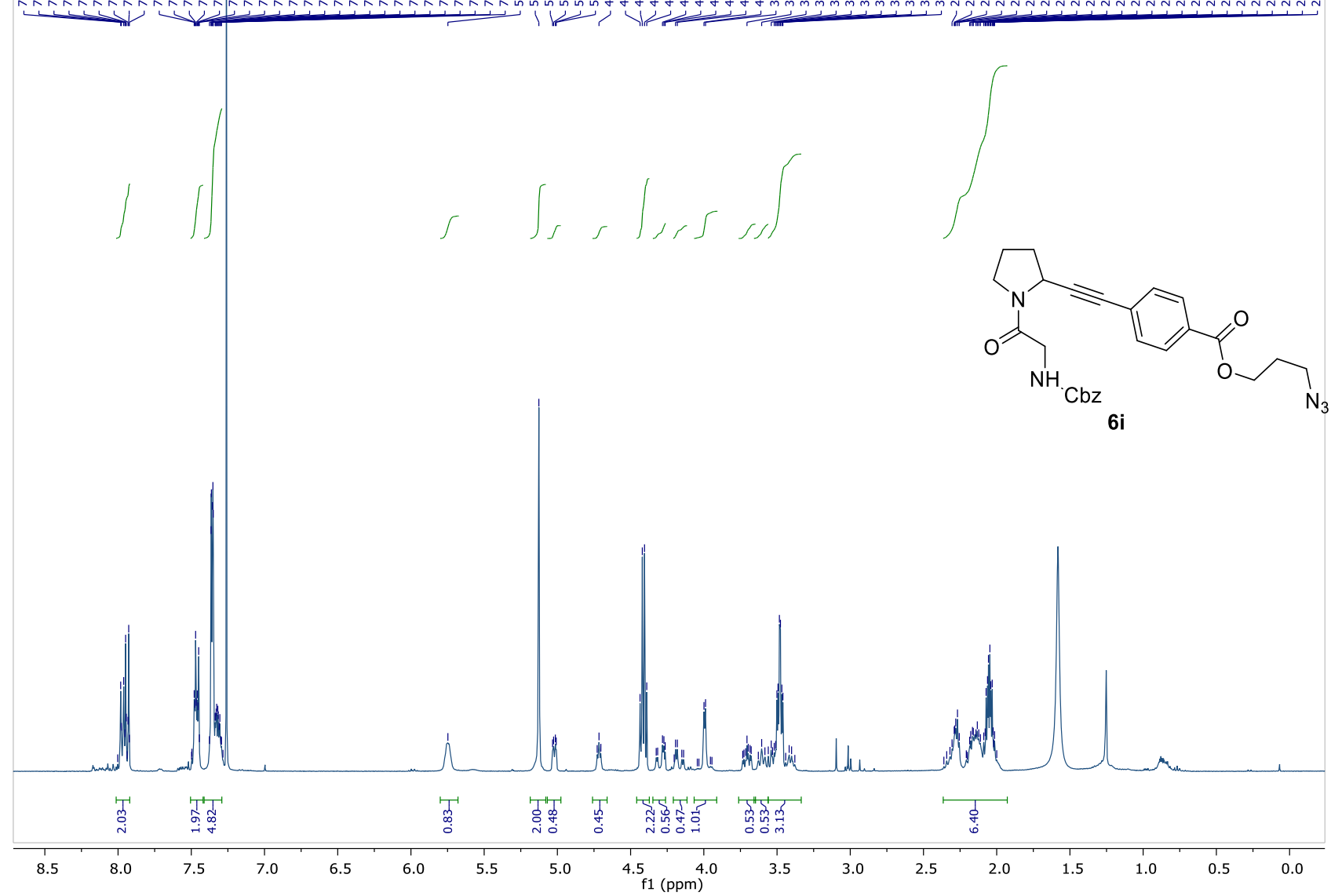

${ }^{13} \mathrm{C}-\mathrm{NMR}(101 \mathrm{MHz}$, Chloroform- $d)$

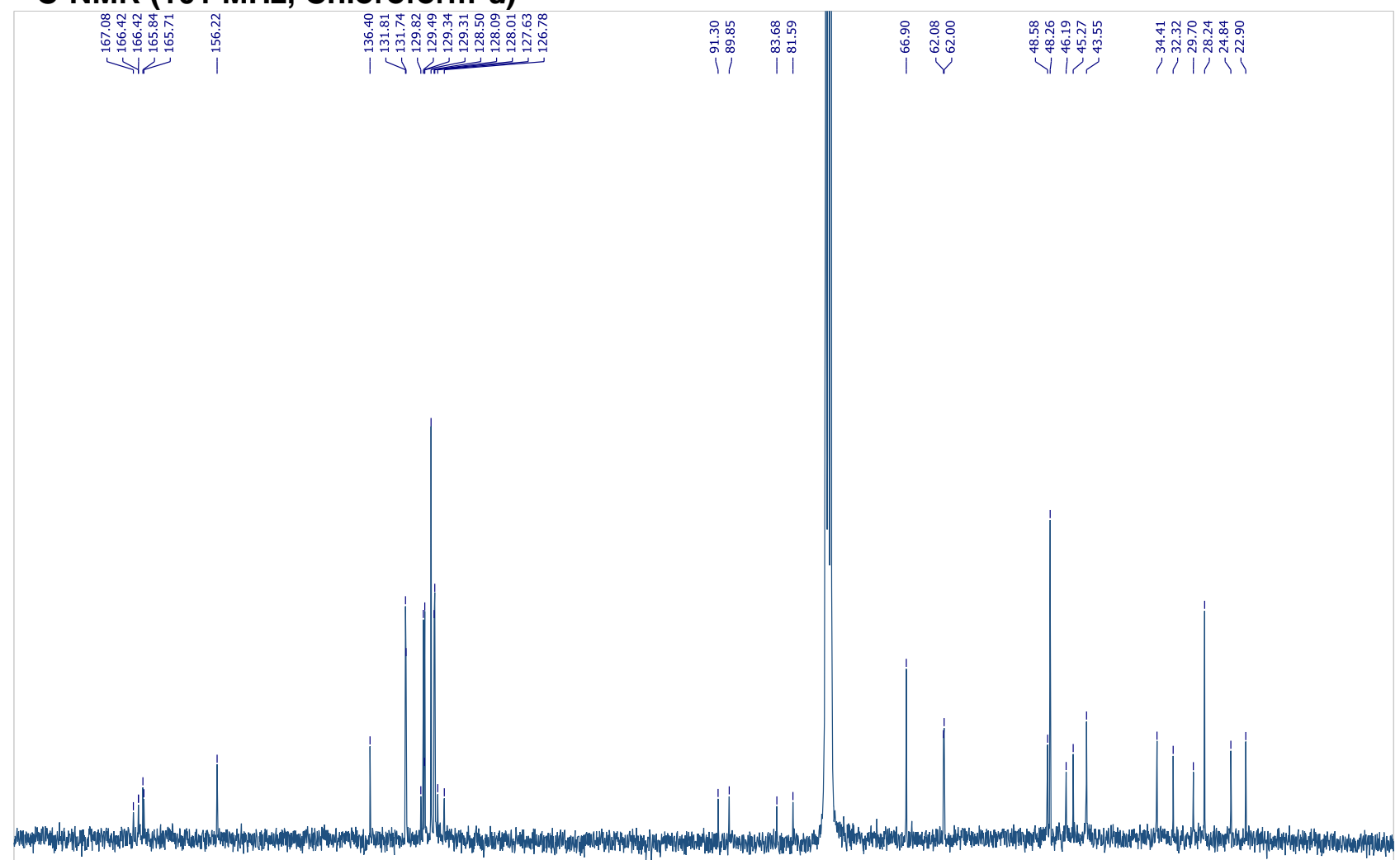

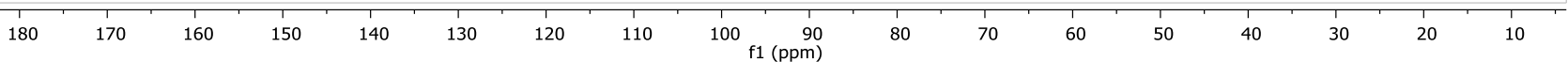


${ }^{1} \mathrm{H}-\mathrm{NMR}$ (400 MHz, Chloroform- $d$ )

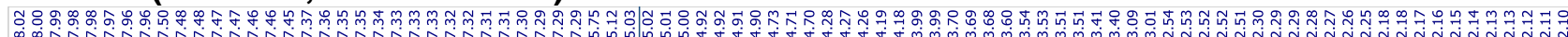

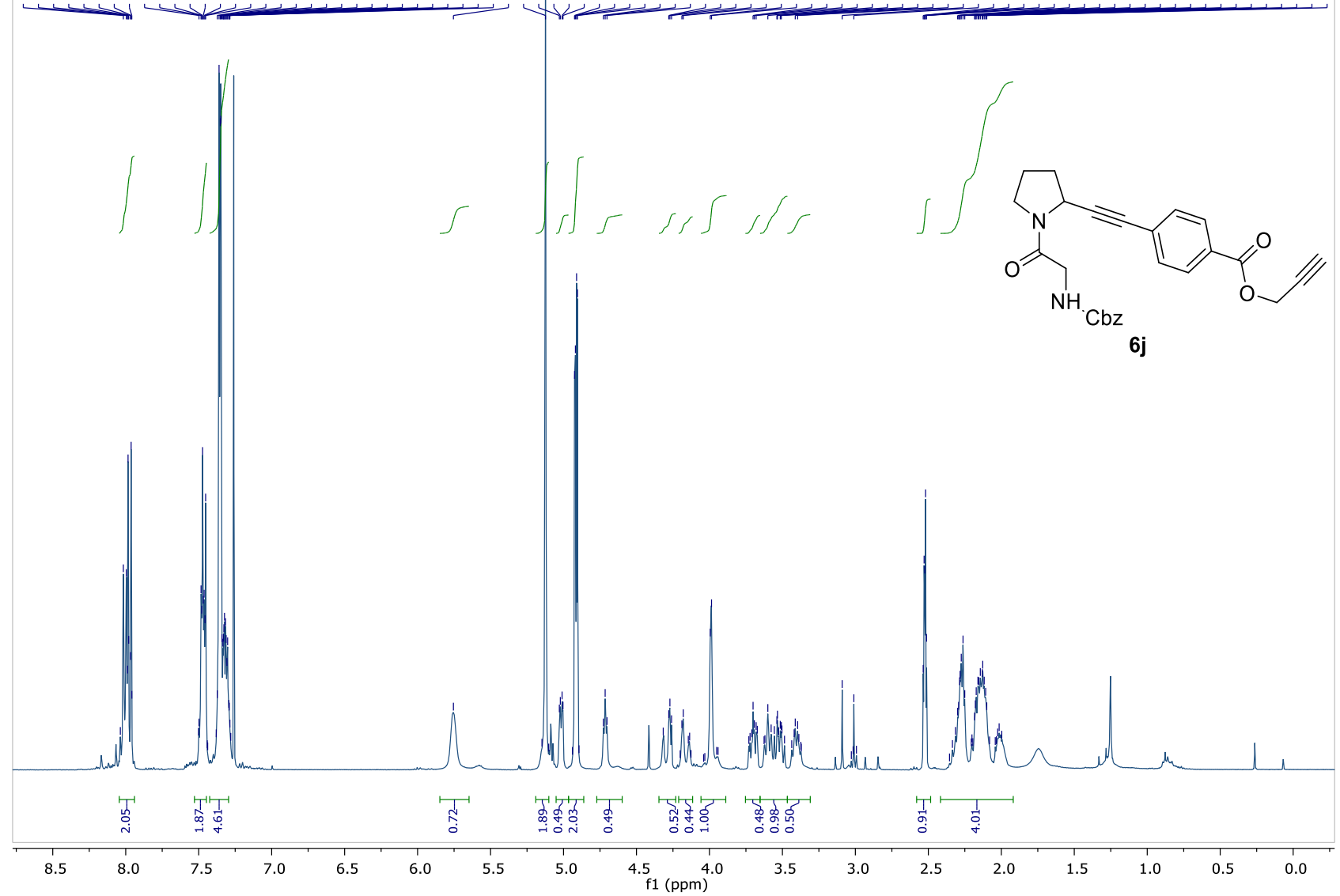

${ }^{13} \mathrm{C}-\mathrm{NMR}(101 \mathrm{MHz}$, Chloroform-d)

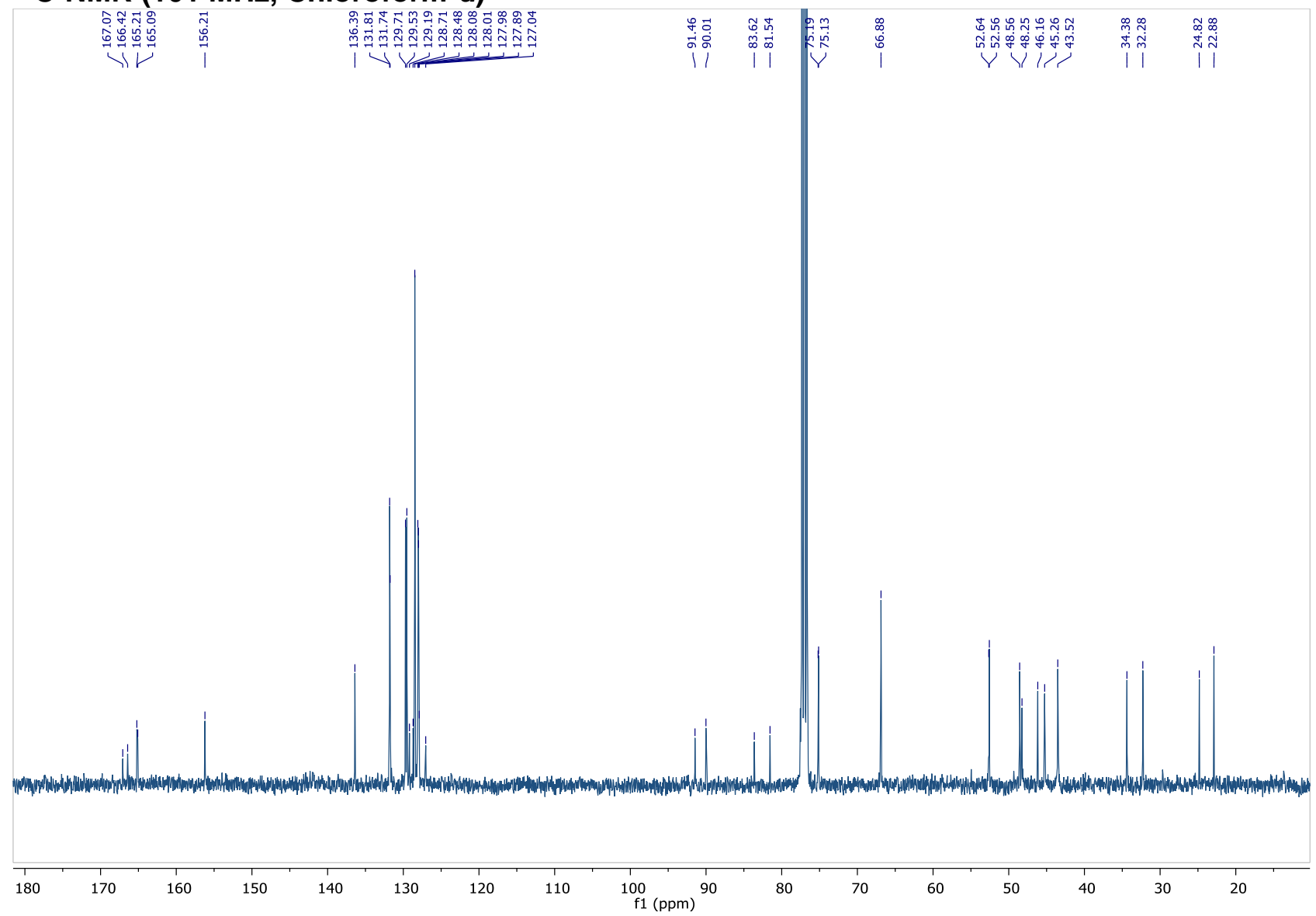


${ }^{1} \mathrm{H}-\mathrm{NMR}(400 \mathrm{MHz}$, Chloroform- $d)$

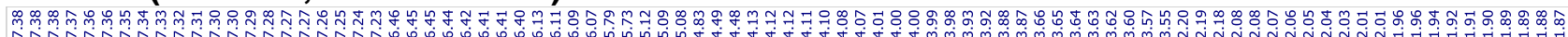

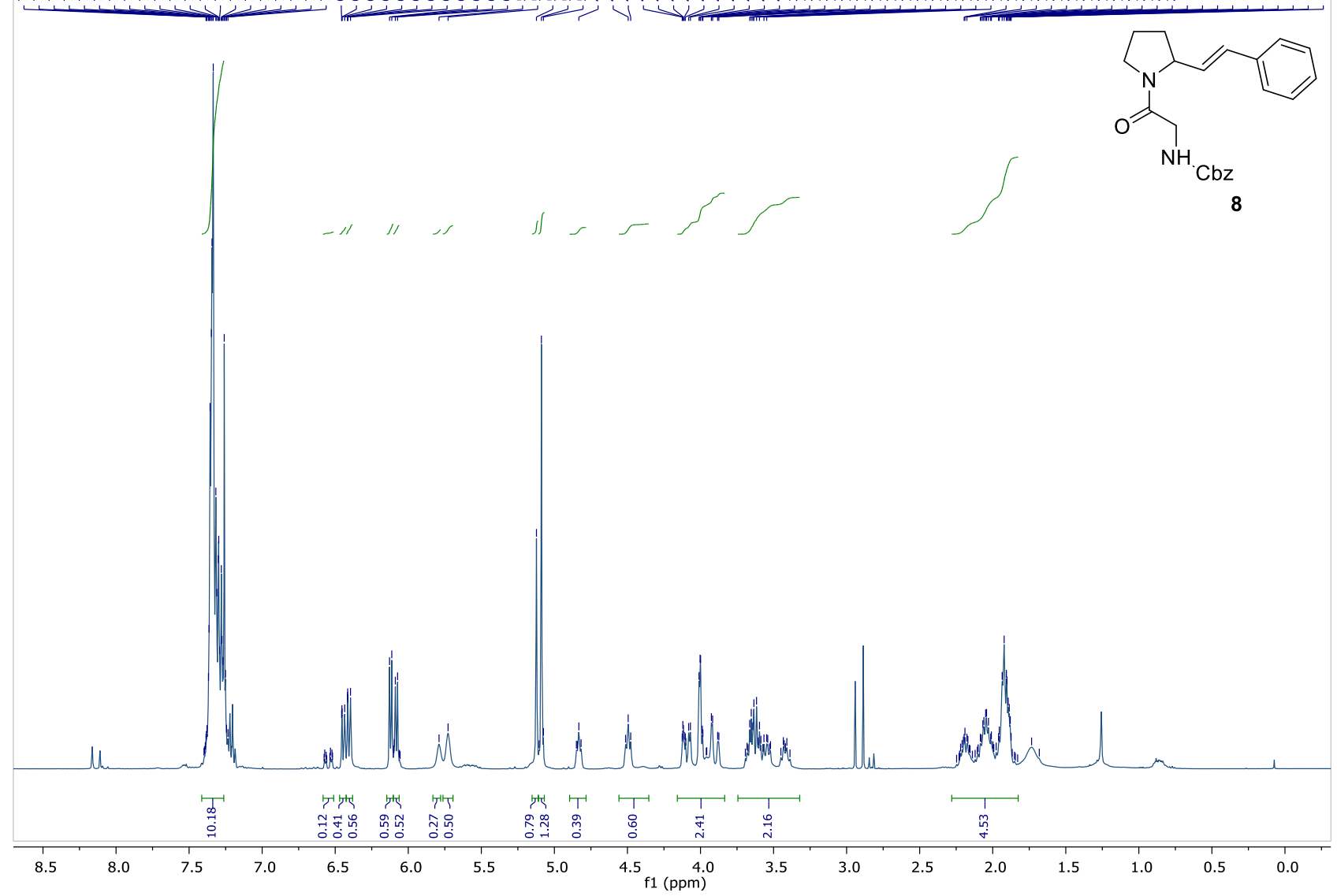

${ }^{13} \mathrm{C}-\mathrm{NMR}(101 \mathrm{MHz}$, Chloroform-d)

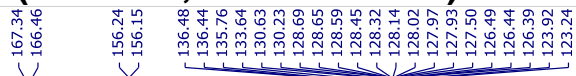

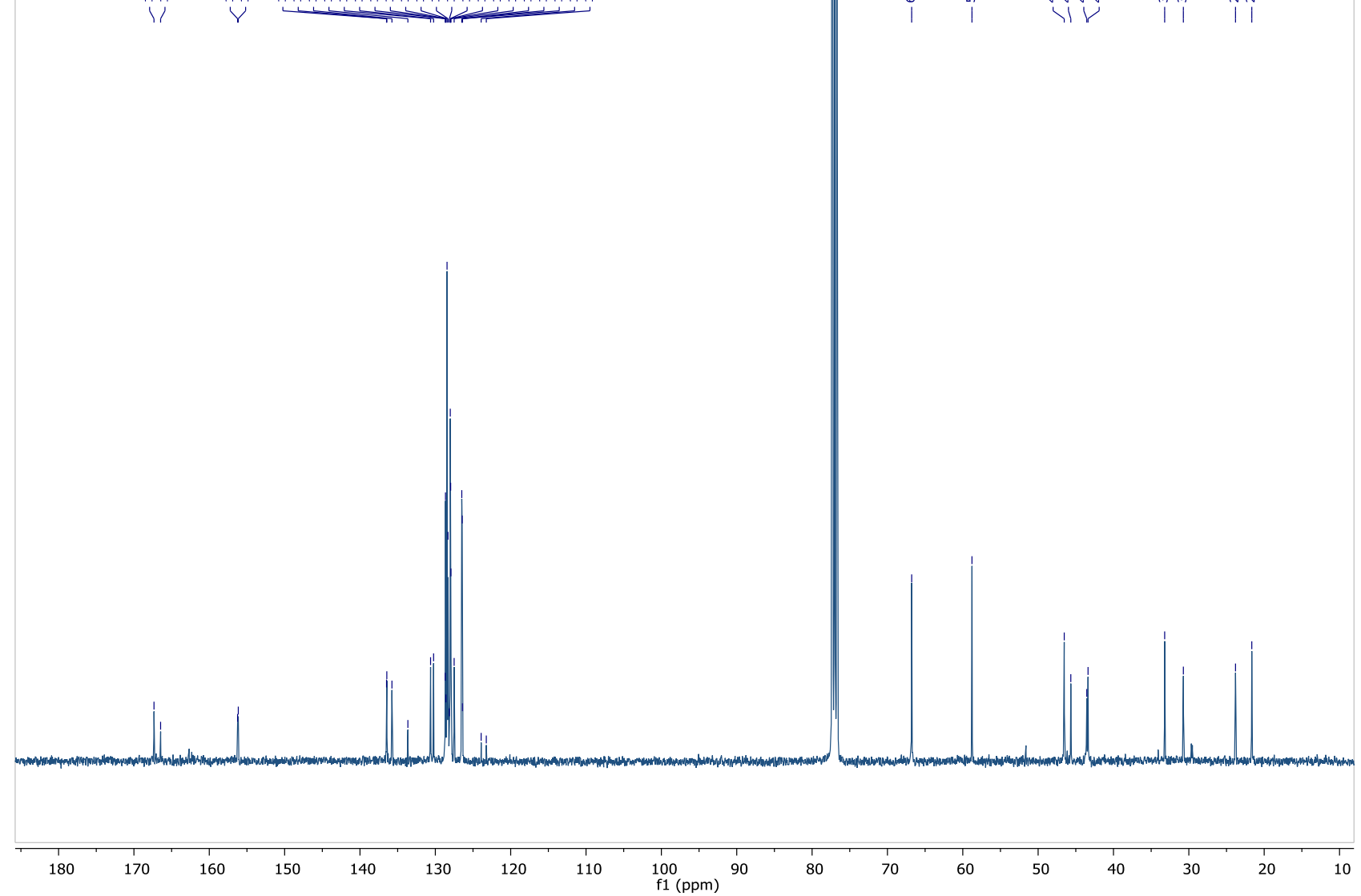


${ }^{1} \mathrm{H}-\mathrm{NMR}$ (400 MHz, Chloroform-d)

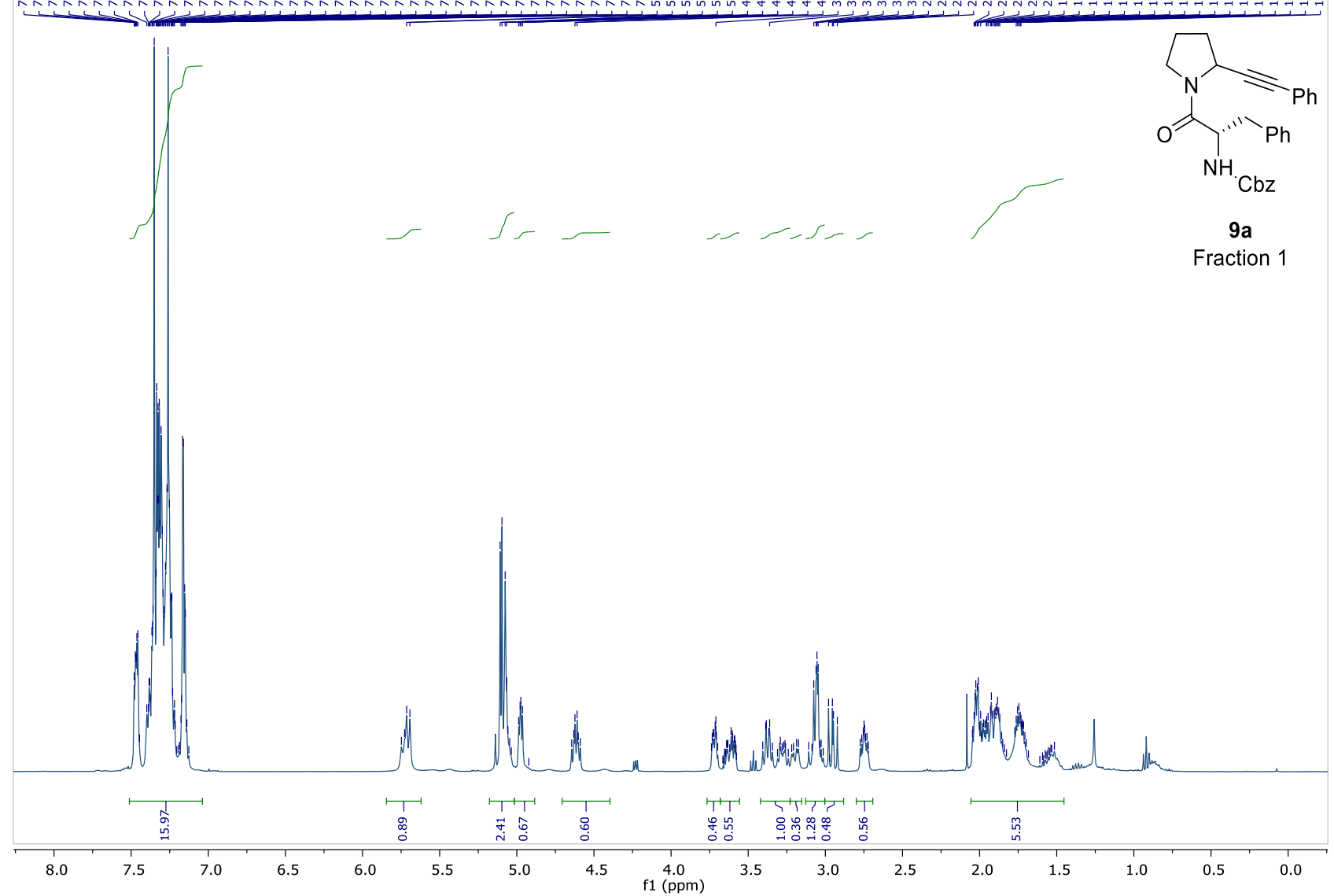

${ }^{13}$ C-NMR (101 MHz, Chloroform-d)

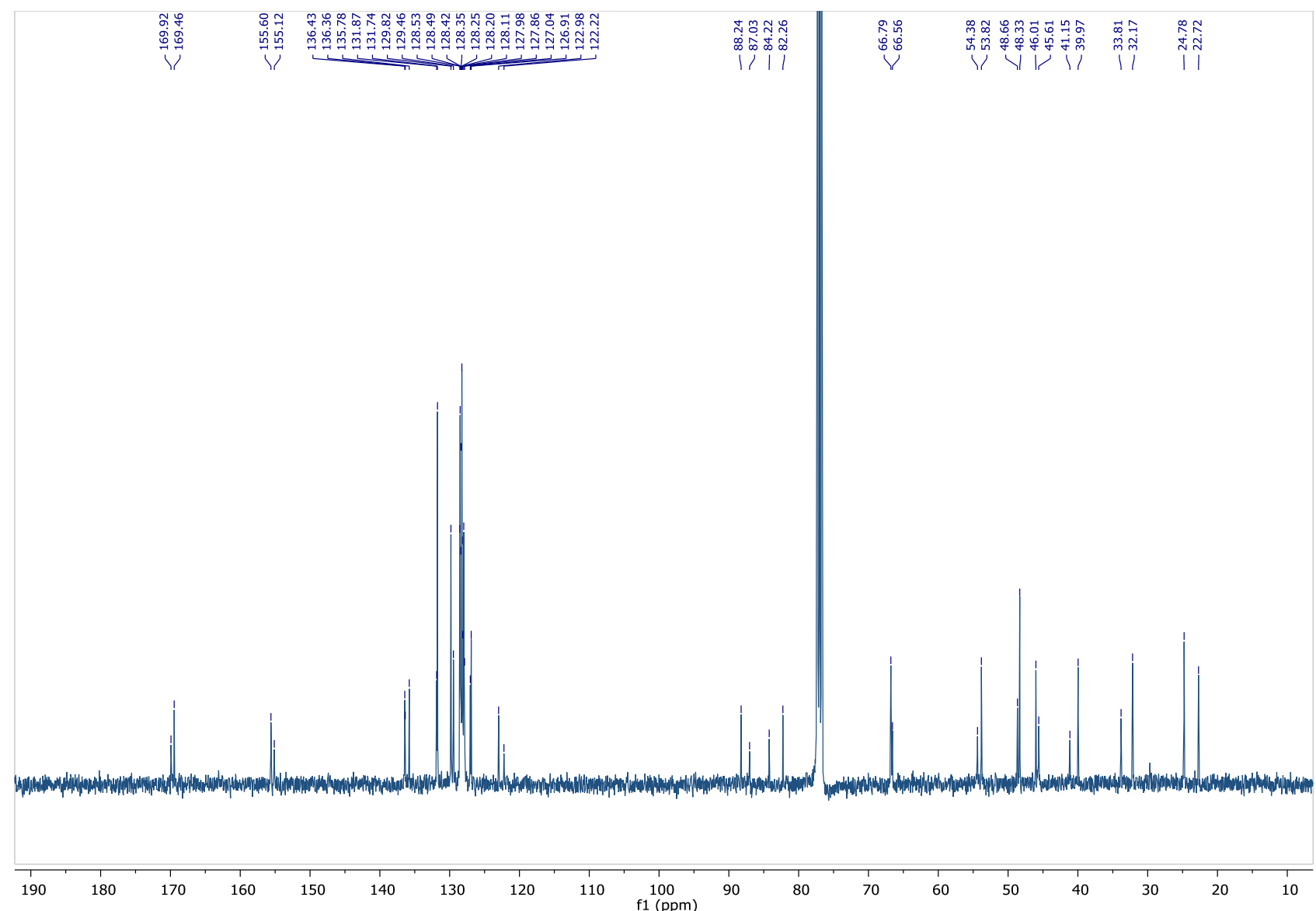


${ }^{1} \mathrm{H}-\mathrm{NMR}(400 \mathrm{MHz}$, Chloroform-d)

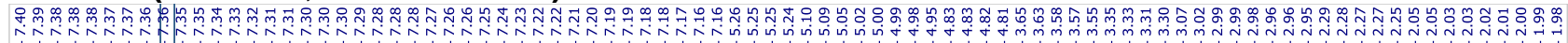

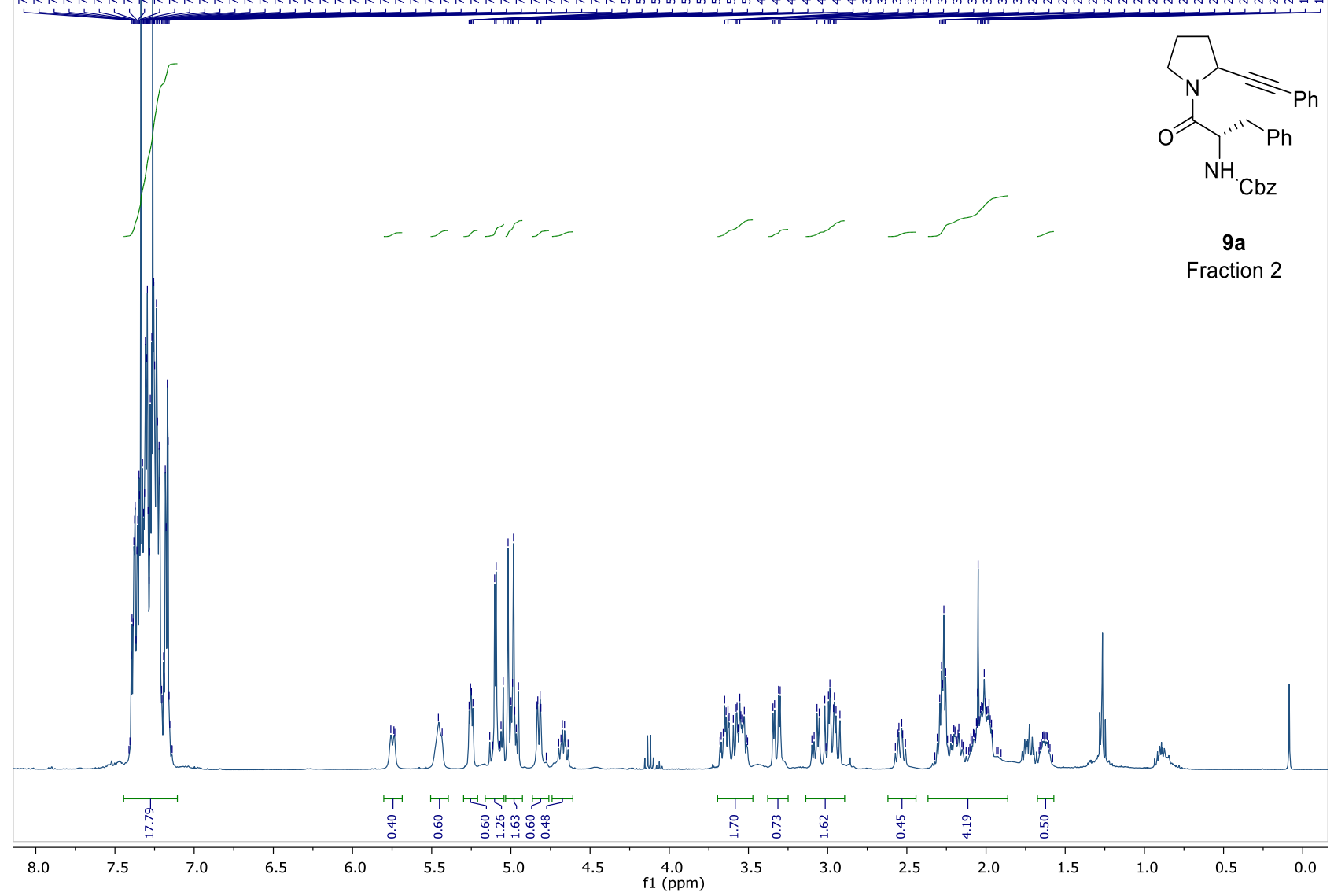

${ }^{13} \mathrm{C}-\mathrm{NMR}(101 \mathrm{MHz}$, Chloroform- $d)$

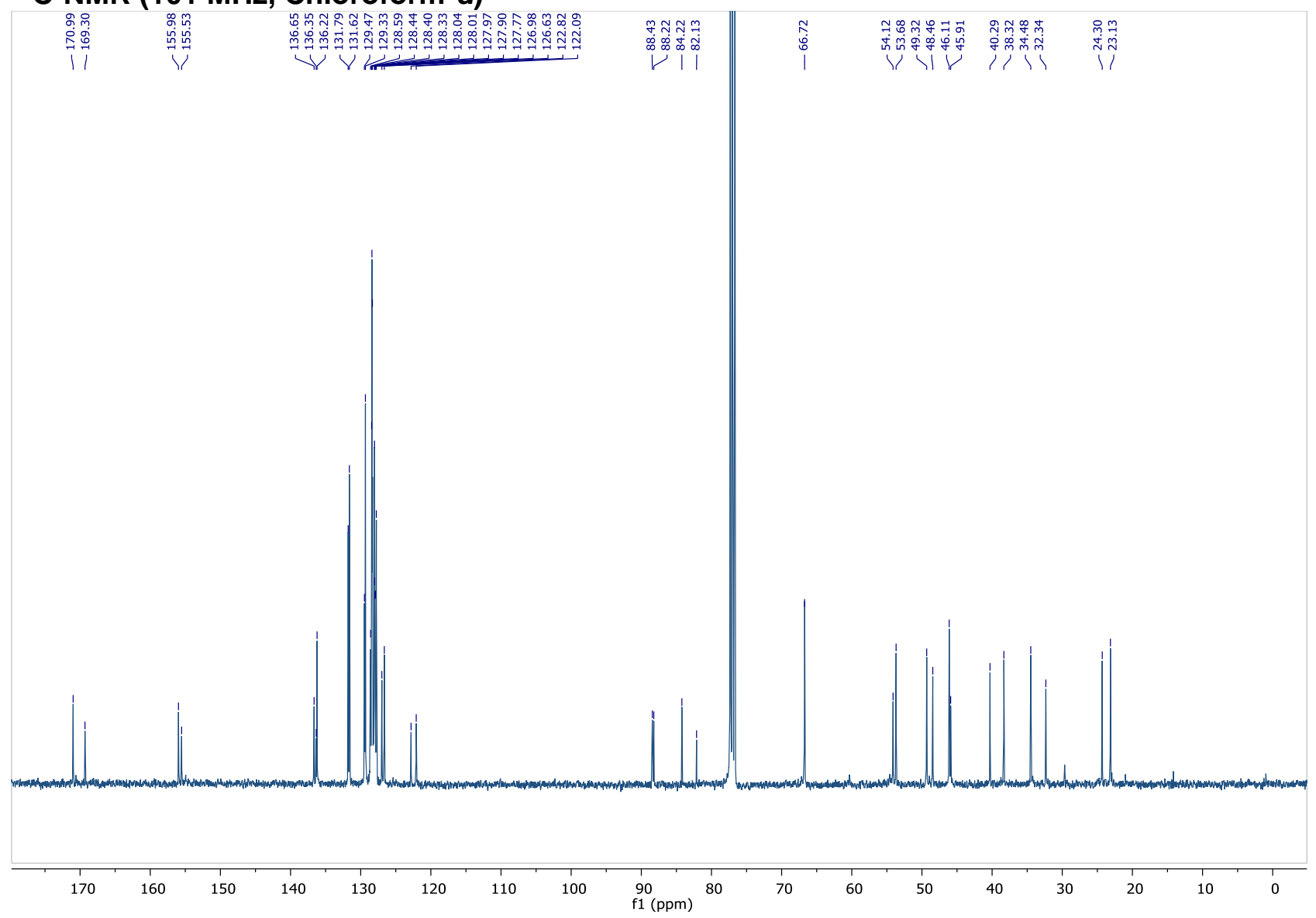




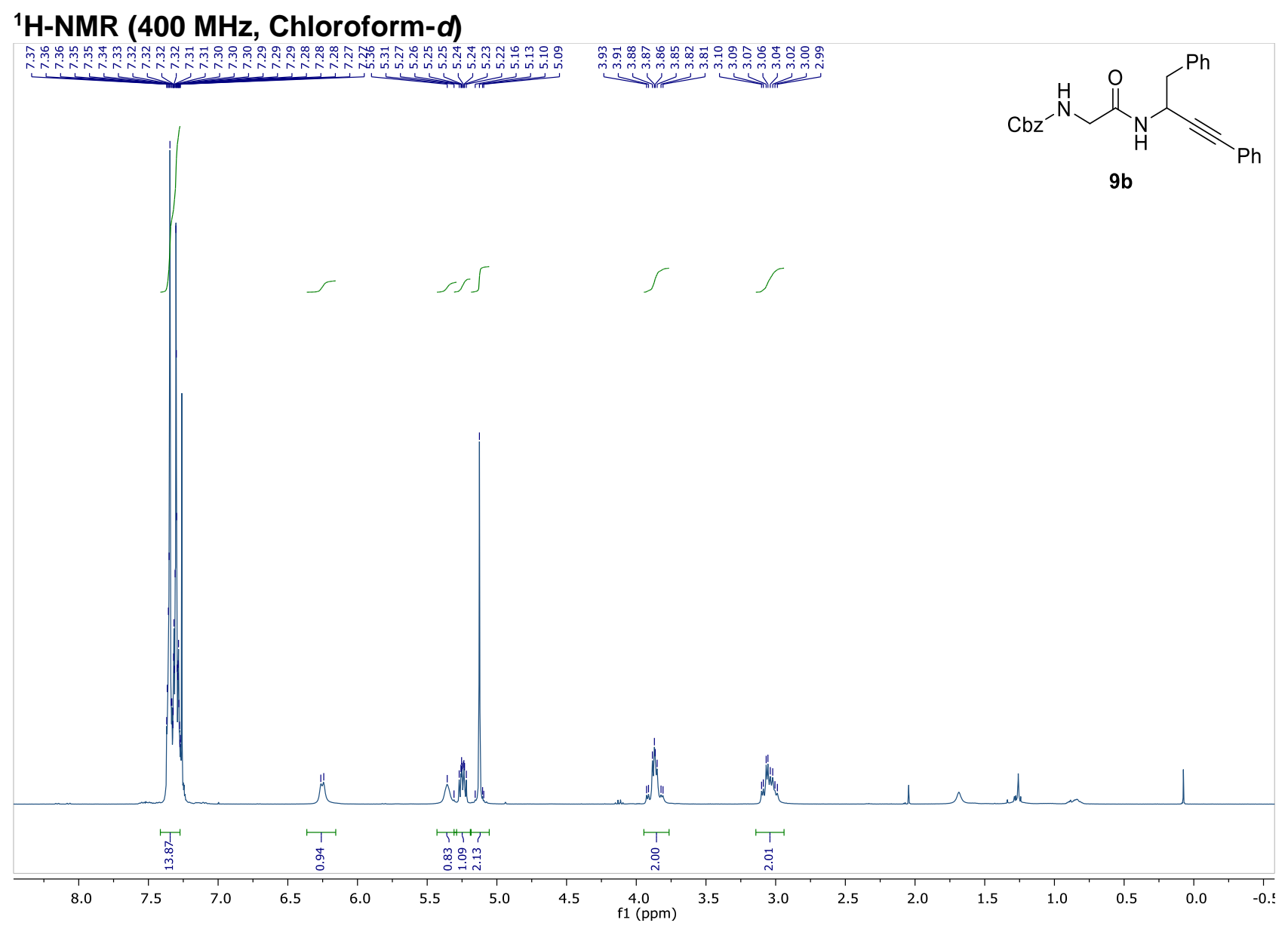

${ }^{13} \mathrm{C}-\mathrm{NMR}$ (101 MHz, Chloroform- $d$ )

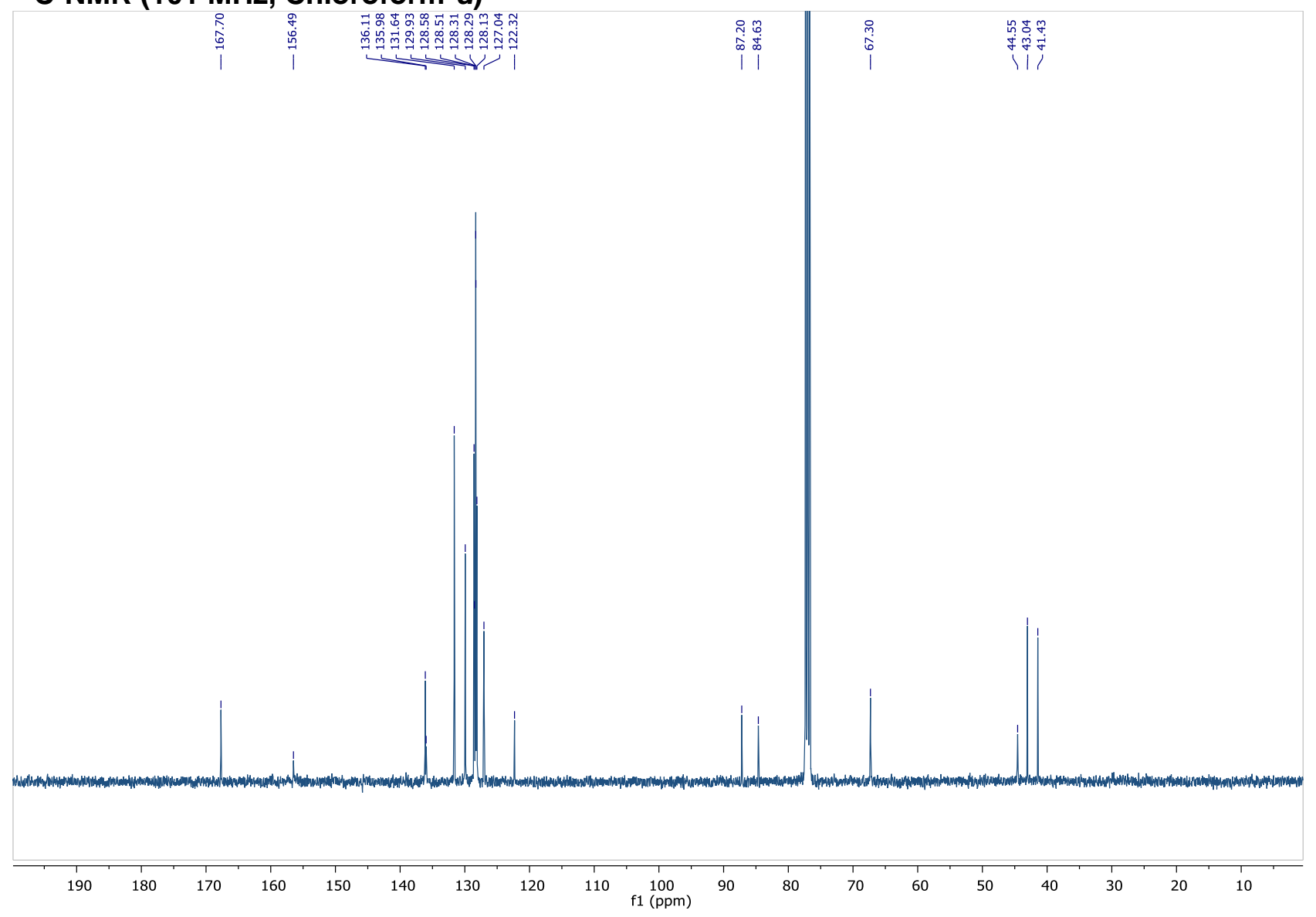

94 
${ }^{1} \mathrm{H}-\mathrm{NMR}$ (400 MHz, Chloroform- $d$ )

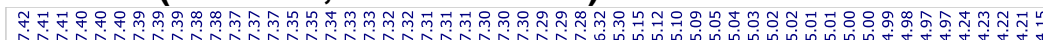

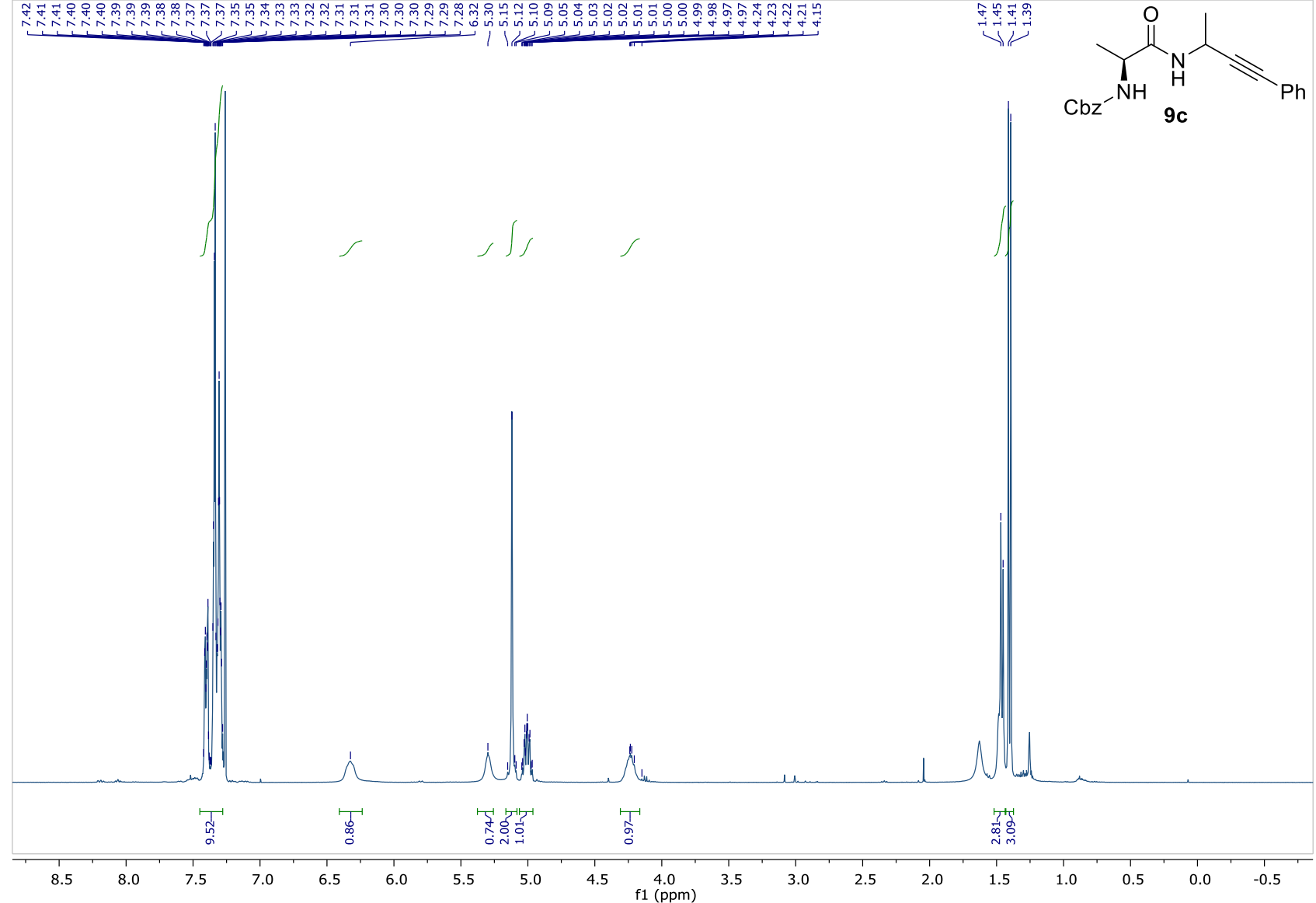

${ }^{13} \mathrm{C}-\mathrm{NMR}$ (101 MHz, Chloroform- $d$ )

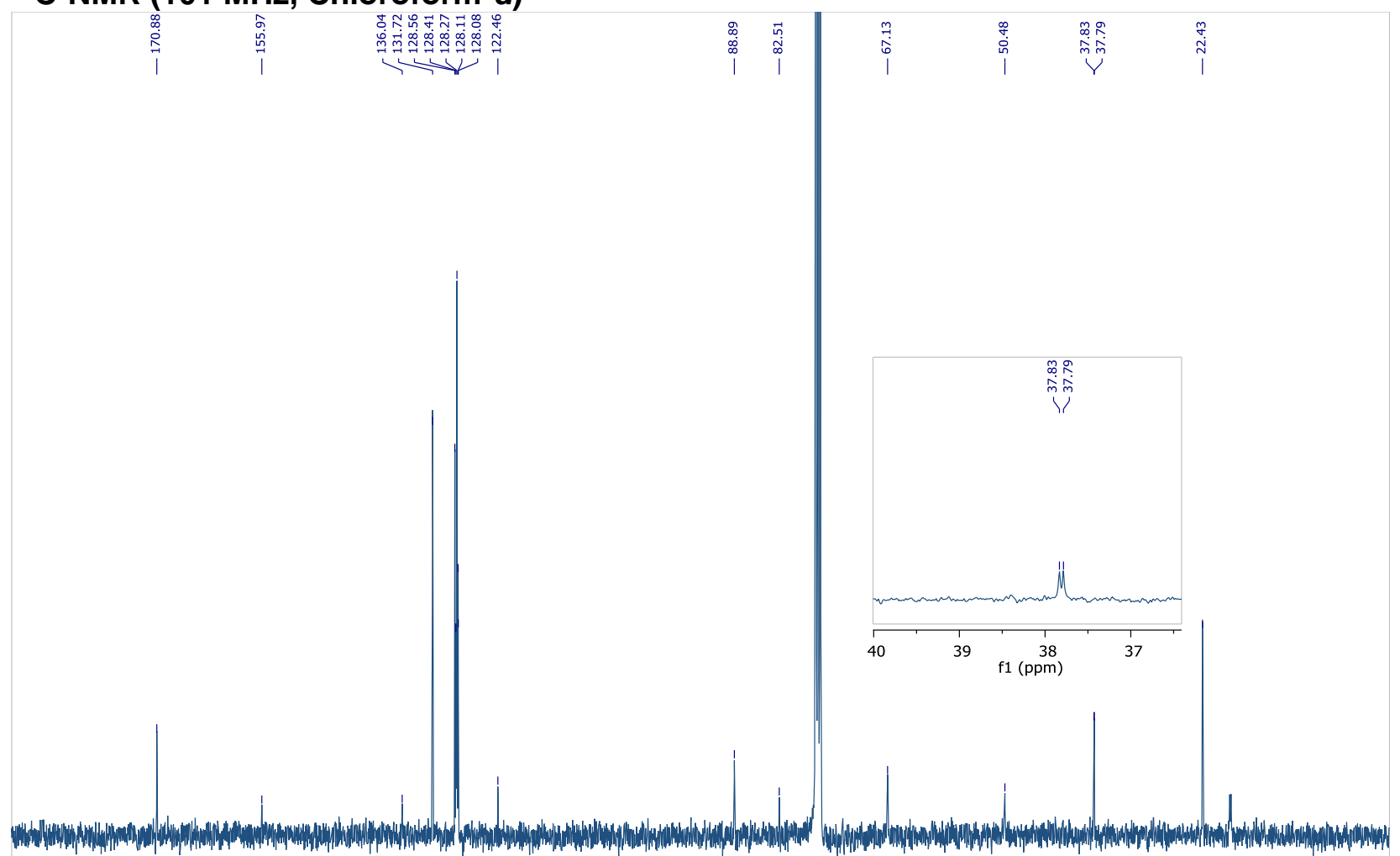

190

180

$\begin{array}{rrrrrrrr}170 & 160 & 150 & 140 & 130 & 120 & 110 & 100 \\ 90\end{array}$

$80 \quad 70 \quad 60$

30 
${ }^{1} \mathrm{H}-\mathrm{NMR}$ (400 MHz, Acetonitrile- $d_{3}$ )

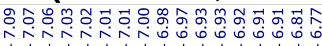

$\underbrace{\infty} \underbrace{\infty}$

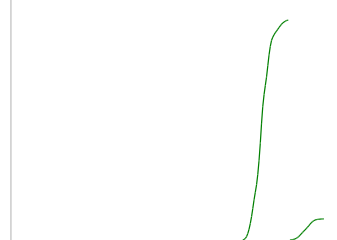

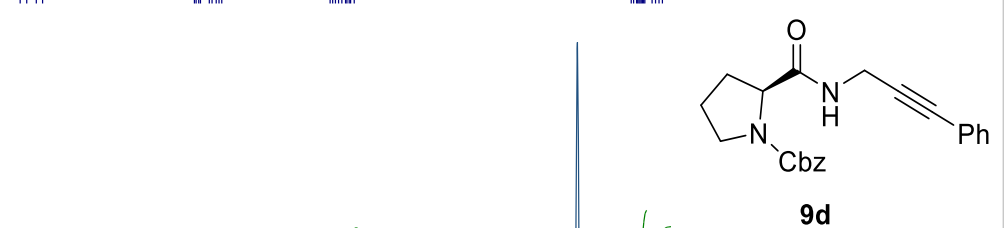
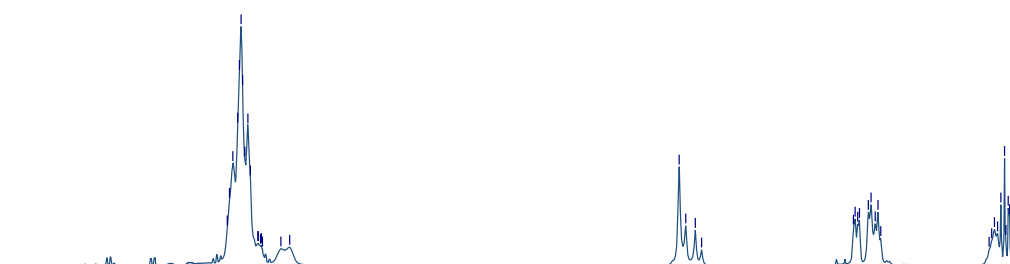

$\int$

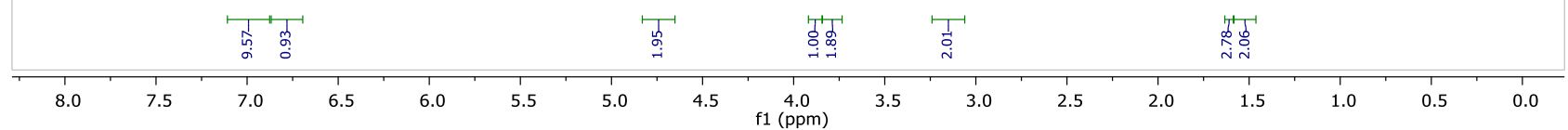

${ }^{13} \mathrm{C}-\mathrm{NMR}$ (101 MHz, Acetonitrile- $d_{3}$ )

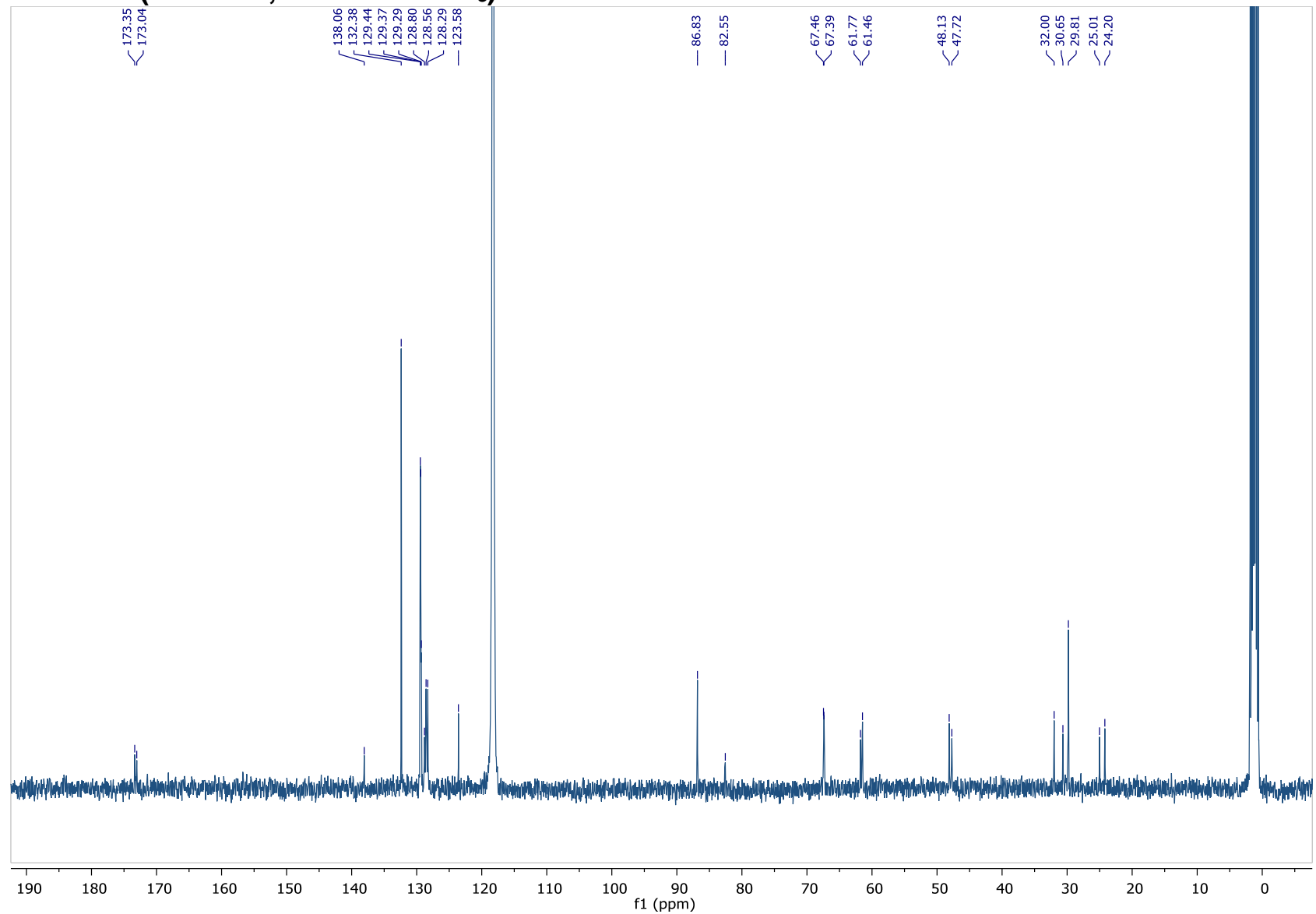


${ }^{1} \mathrm{H}-\mathrm{NMR}$ (400 MHz, Chloroform- $d$ )
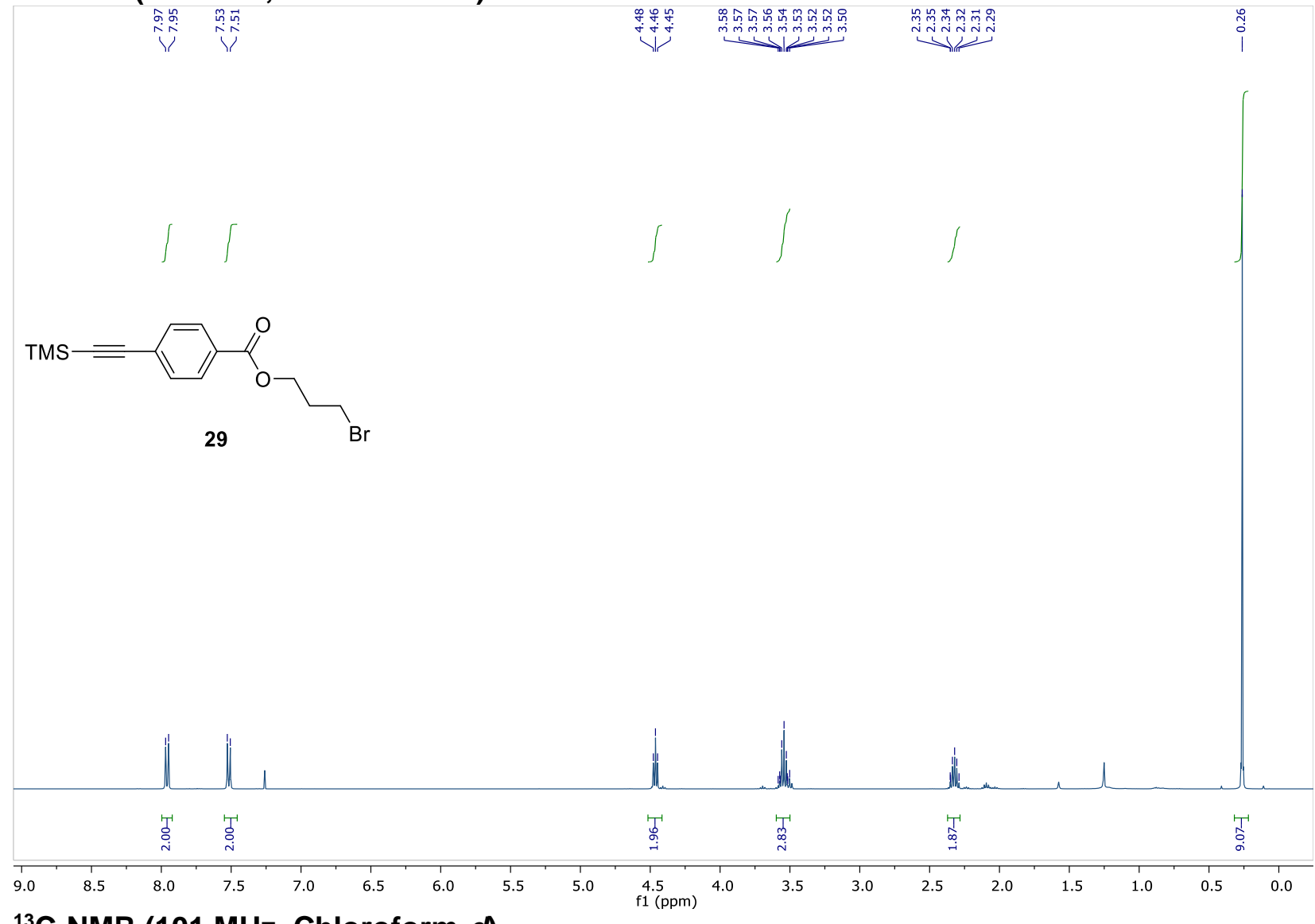

${ }^{13} \mathrm{C}-\mathrm{NMR}$ (101 MHz, Chloroform- $d$ )

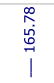

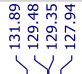

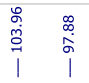

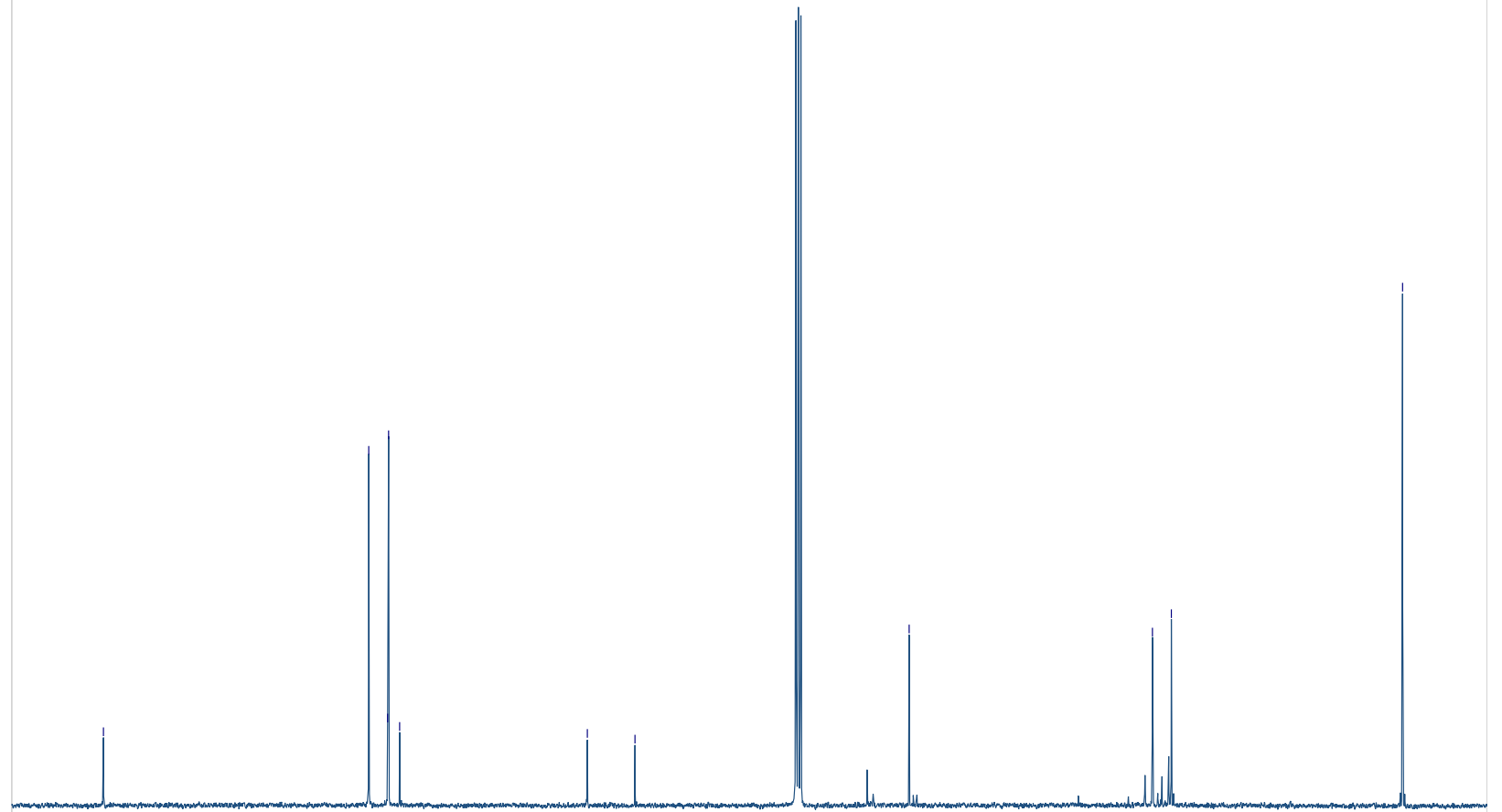

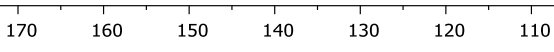
100 $\begin{array}{rr}90 & 80 \\ \mathrm{f} 1(\mathrm{ppm})\end{array}$ ${ }^{1} \mathrm{H}-\mathrm{NMR}$ (400 MHz, Chloroform-d) 


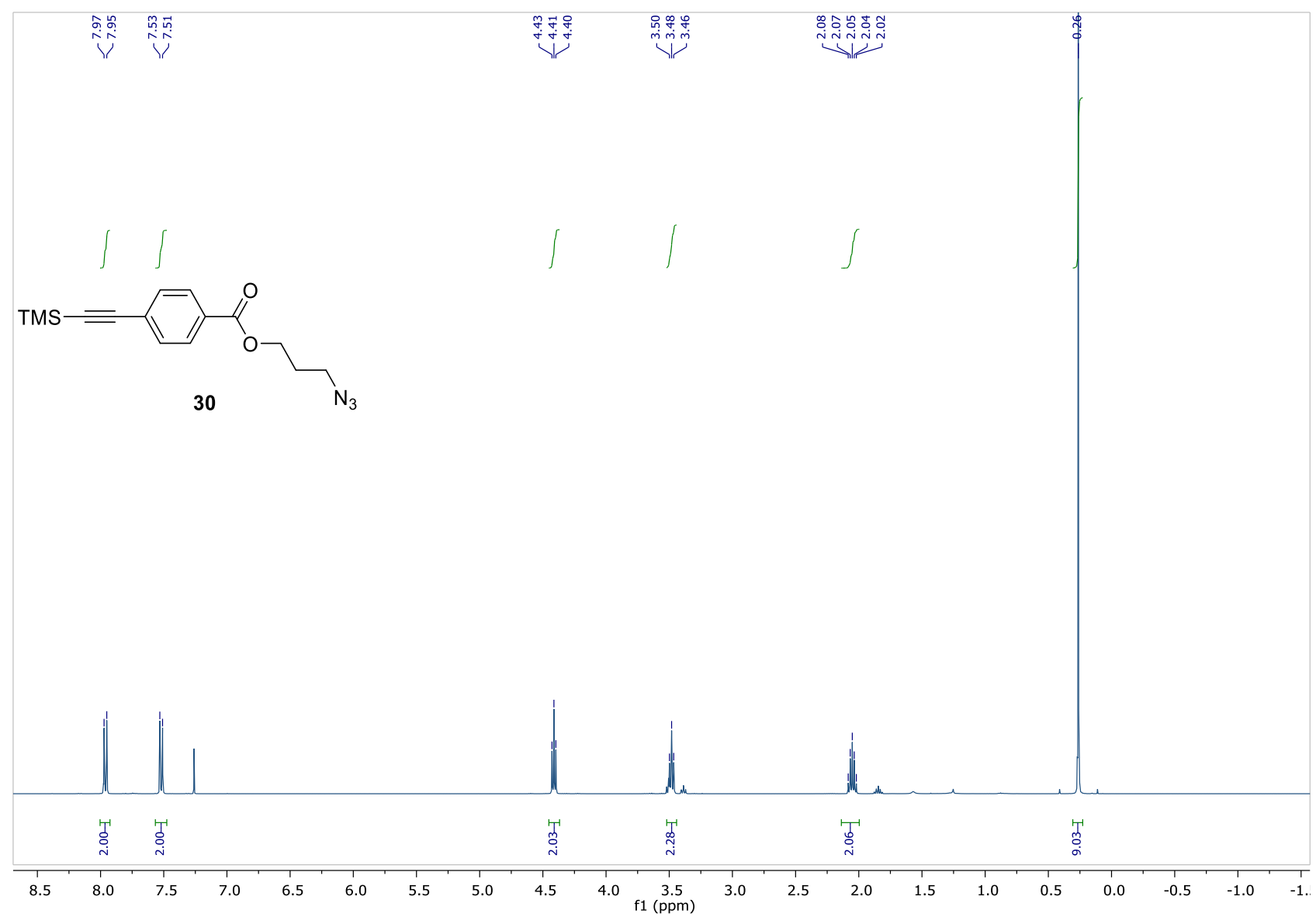

${ }^{13} \mathrm{C}-$ NMR (101 MHz, Chloroform- $d$ )

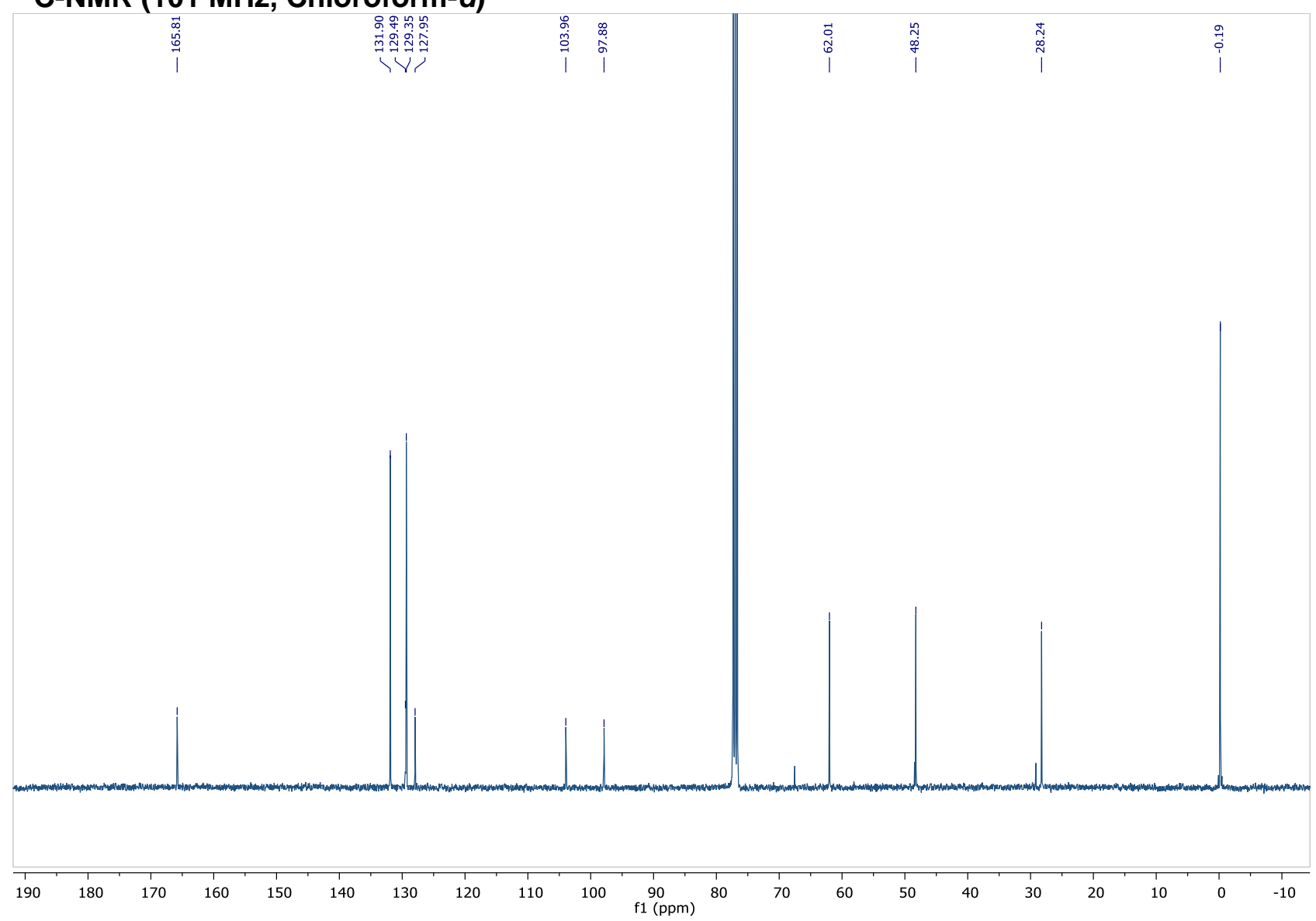




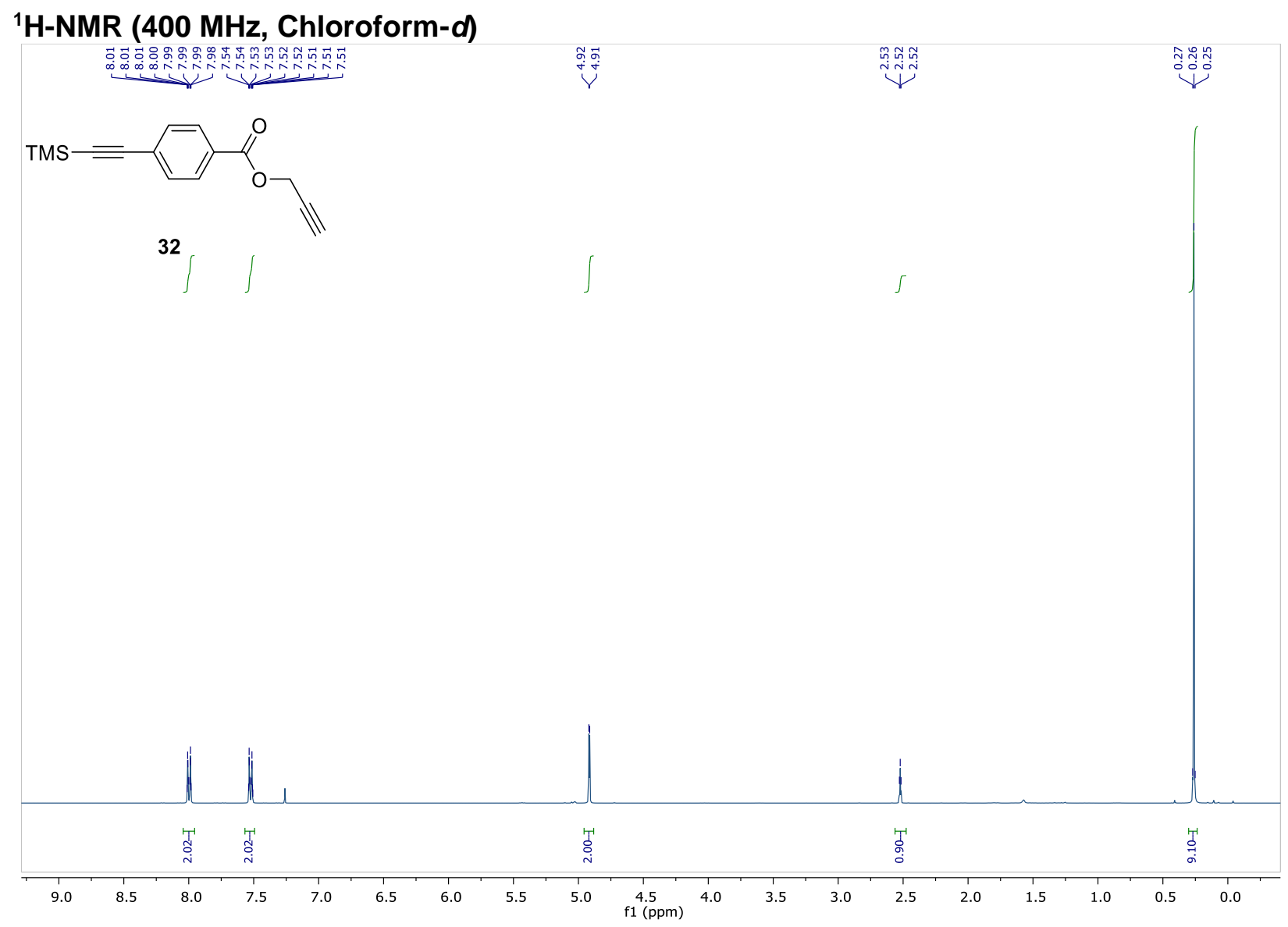

${ }^{13} \mathrm{C}-\mathrm{NMR}$ (101 MHz, Chloroform- $d$ )

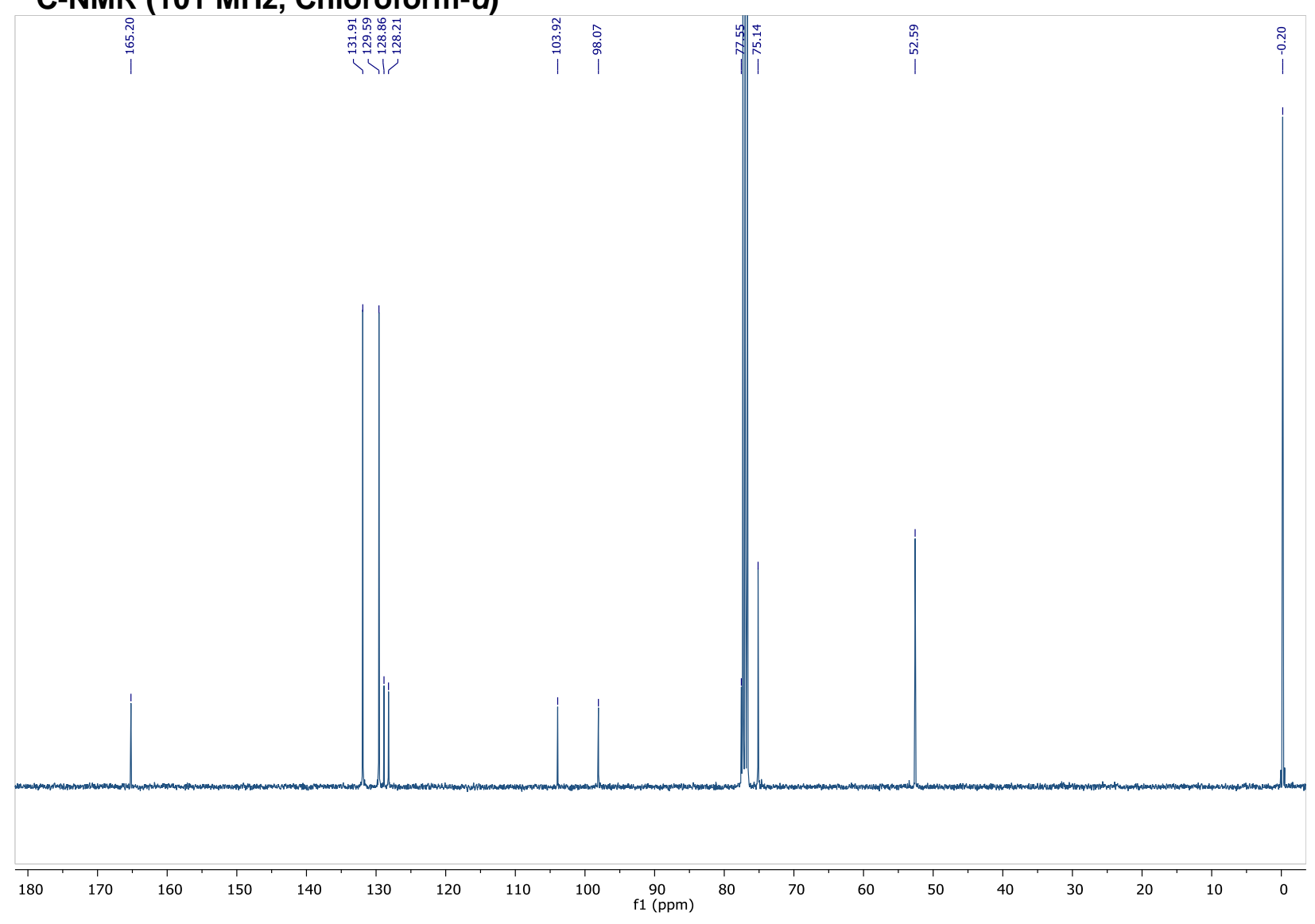

99 
1H-NMR (800 MHz, DMF- $\left.d_{7}\right)$

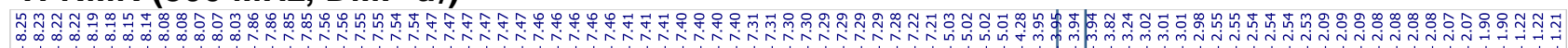

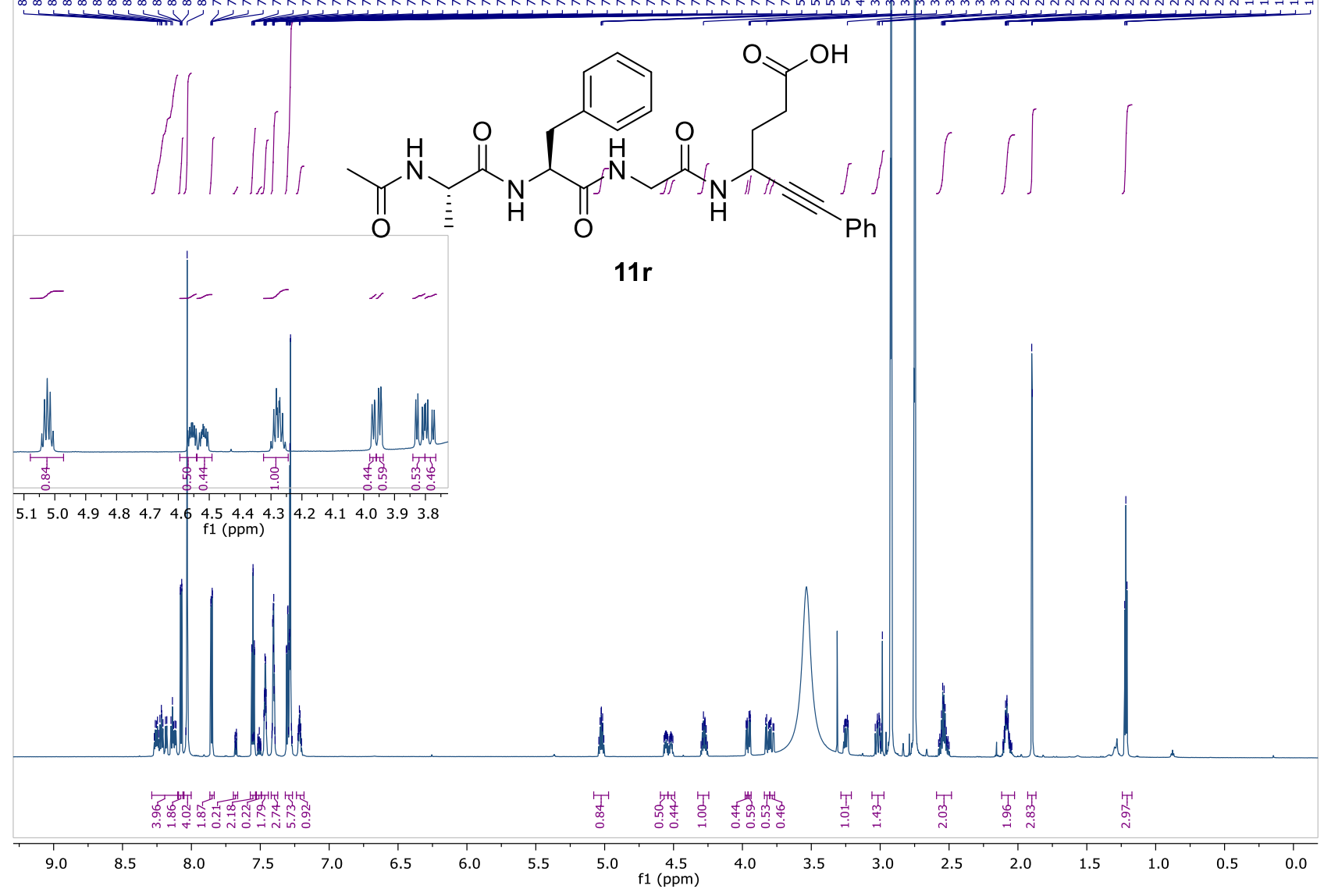

${ }^{13} \mathrm{C}-\mathrm{NMR}\left(200 \mathrm{MHz}, \mathrm{DMF}-d_{7}\right)$

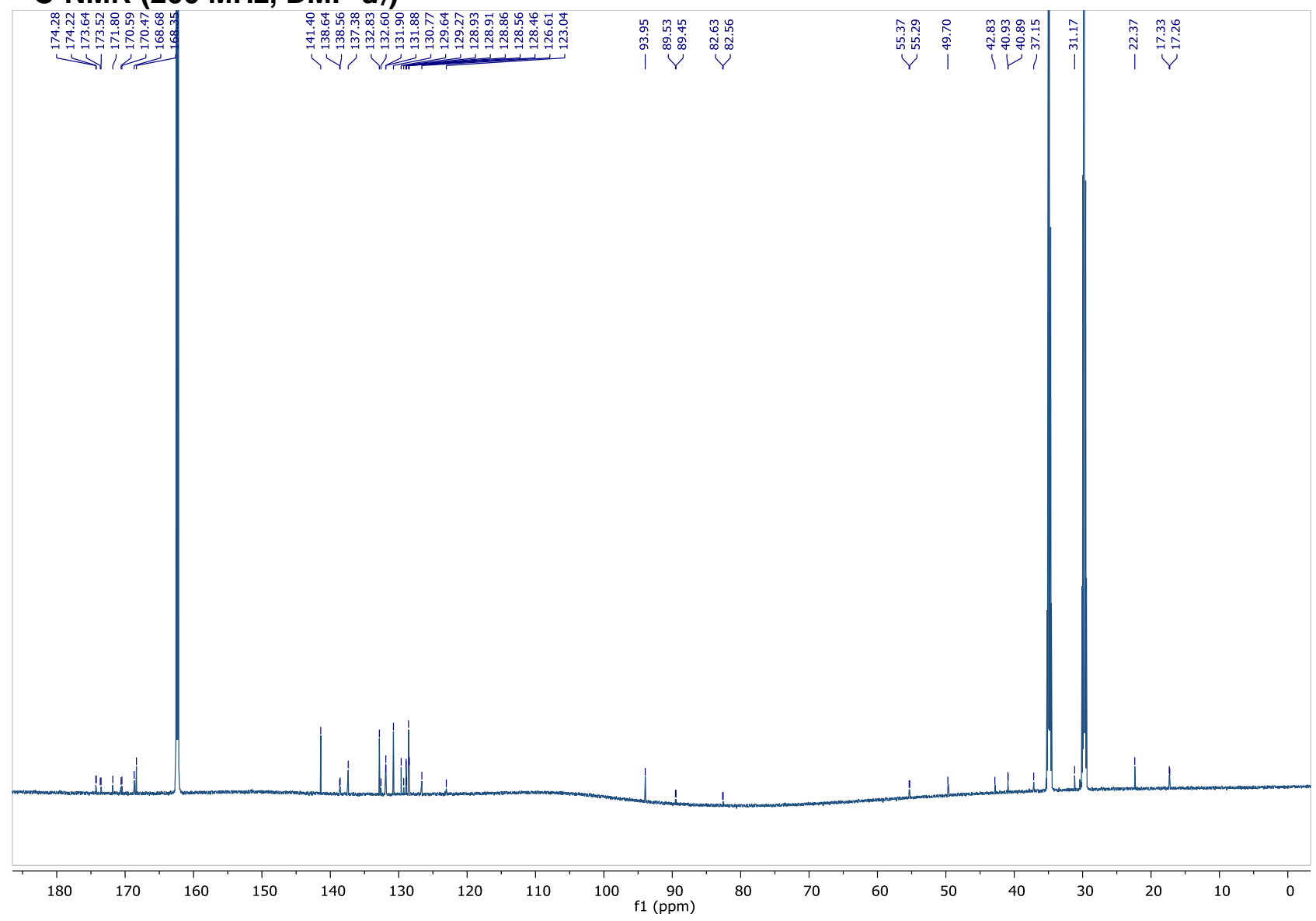


HMBC (DMF- $\left.d_{7}\right)$

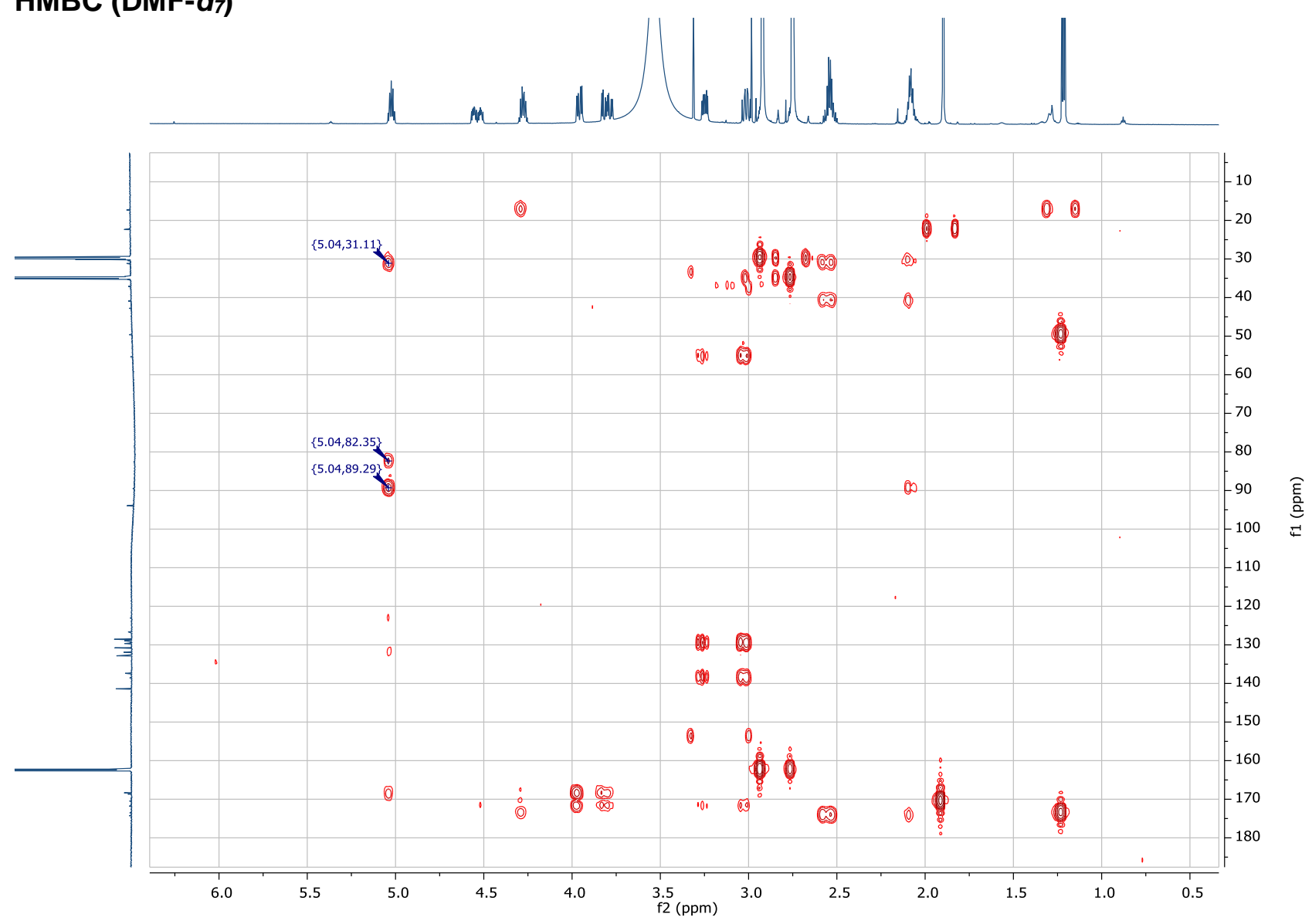


${ }^{1} \mathrm{H}-\mathrm{NMR}\left(800 \mathrm{MHz}, \mathrm{DMF}-\mathrm{d}_{7}\right)$

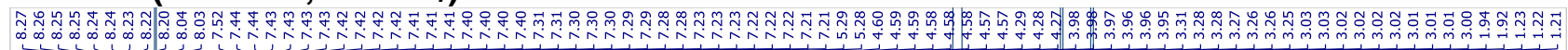

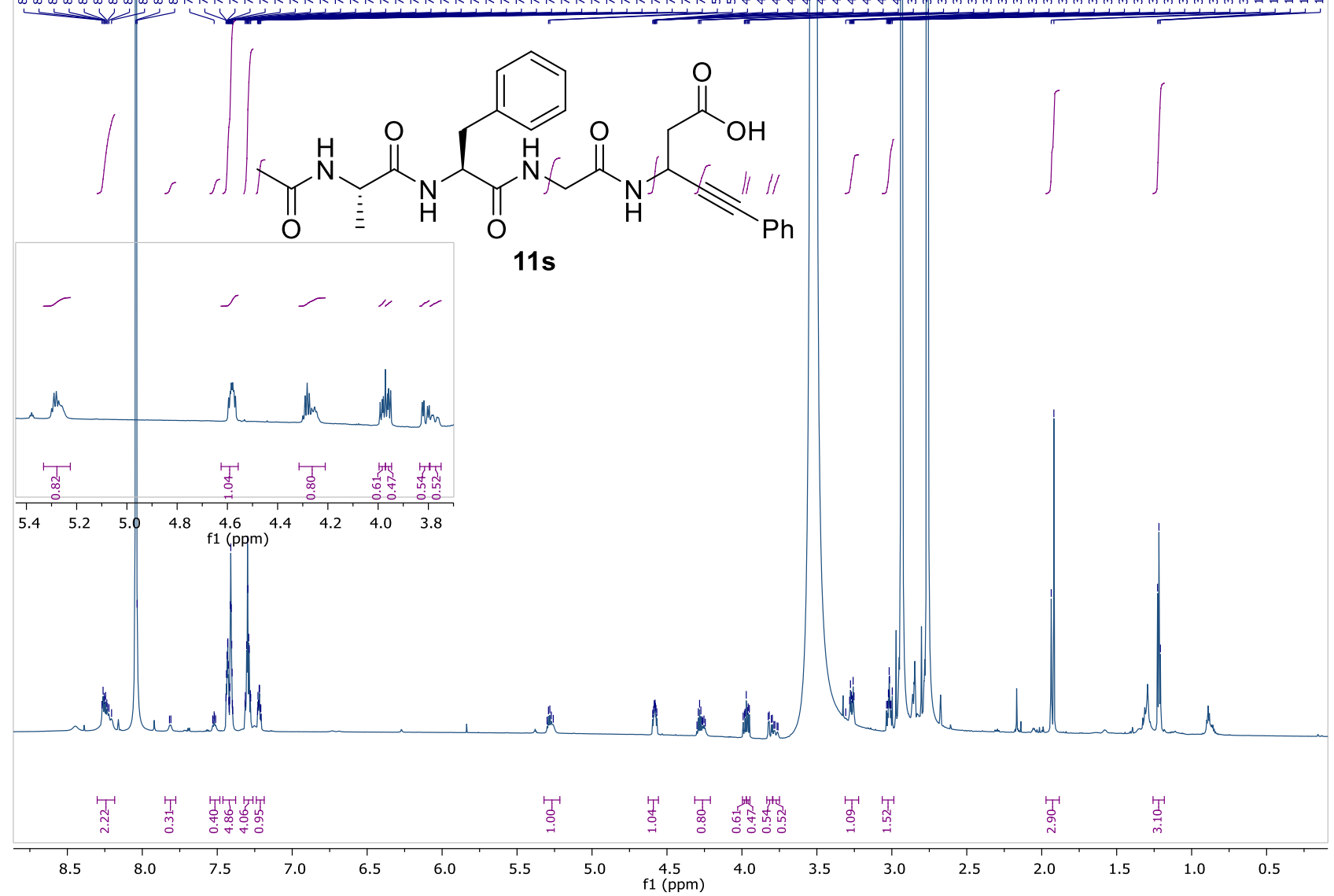

